
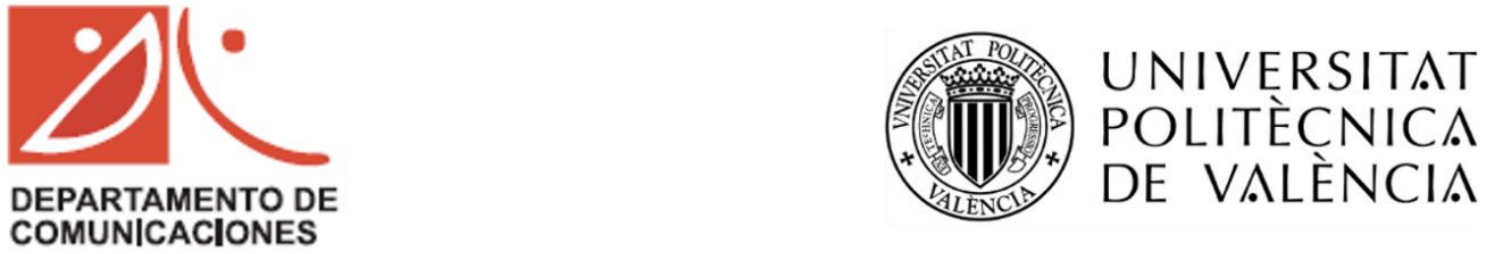

Universitat Politècnica de València

Departamento de Comunicaciones

\title{
Development of high sensitivity
}

\section{photonic sensing structures based on}

\section{porous silicon substrates}

\author{
Raffaele Caroselli
}

Supervisor: Dr. Jaime García Rupérez

Thesis submitted to the Universitat Politècnica de València in partial fulfilment of the requirements for the degree of Doctor of Philosophy in Telecommunications engineering. 



\section{Acknowledgements}

It is a pleasure and cause for gratitude to acknowledge those who have contributed directly or indirectly to make this $\mathrm{PhD}$ Thesis possible.

I would like to start by acknowledging my supervisor Jaime. You welcomed me so I could develop my MSc degree final project in the NTC and after that you believed in me and offered me the great opportunity to stay and start what became this wonderful experience of 4 years. You led me to give all my best and to reach this incredible goal. Thank you sincerely for everything. I would also like to thank the director of NTC for allowing me to realize my $\mathrm{PhD}$ Thesis in this center.

A special thank is for Javi. You have been the first colleague I had in the NTC and you taught me the basis of the optical biosensing world. Furthermore, and mainly, you are the first friend I made in Valencia and who took well care of me.

I want to acknowledge the Biophotonics group as well, namely Salva, Ángela, David, Paula, Jad and Luis. Specially, the porous silicon team, Salva and David, whose help has been crucial in order to obtain such extraordinary results. Salva, you taught me how a real engineer takes action. Thank you very much. Likewise, I have to thank the amazing fabrication and equipment teams: Amadeu, Laurent, Juan, Dora, Jose, David, Luis, Cristina and Alfredo. Thank you Amadeu, for fabricating all my chips, Jose, for all your wise advice, David and Luis, for always helping me to solve all the practical issues. You all are awesome.

Besides, I would like to express my sincere gratitude to all my colleagues and also friends of the open room. In particular, I want to thank my best no-friend Pau, an amusing and thoughtful friend who provided me with all kind of meaningful conversations; Julio, the best computer technician I could have, who never said no to my continuous demands; Carles, a great chef and a better friend, always willing to listen to me; Álvaro, the strongest man I have ever met, Luis, a kind guy who helped me in many occasions and, of course, Marghe, the smiling girl with whom I had the luck of sharing this time. Thank you guys, you made these years amazing. 
Also several people external to the NTC have been with me during these years. For this reason, I want to thank all of them. Specially Rosario, who received me and took care of me like a son; Álvaro, for encouraging me without stopping; Toni and Gracia who have been by my side in the hardest moments. I needed all of you and I really appreciate everything you have done for me.

I would like to show my deepest gratitude to my family. Thanks to my parents, Franco and Maria Grazia. You have been the first teachers I have had in my life and from you I learnt not only to work hard and to be a good student, but mainly to live, to love the neighbour and to be a good person. I owe you everything. Thanks to my brothers Stefano, Paolo and Lorenzo and to my little sister Chiara Susanna. Your support has been essential in this years.

Finally, I want to thank Ángela, for being the pillar where I could lean when I was weak, the shelter where I could find the strength when I was feeling down and the energy that pushed me to never give up. You motivated me every day to do my work as well as possible. Thank you for standing by me during this period. 


\section{Abstract}

Health and well-being have always been the center of attention of many research institutions and companies around the world. This led the technology to develop in the chemical, biological, medical and clinical fields with the aim to provide a better protection to the human being. As a consequence, a competition is born between the time necessary to the disease to progress and the time necessary to man to treat such disease. In order to win this competition, it is necessary to act with anticipation, when disease is not too developed yet. This is possible by performing an early-detection. The achievement of this goal paves the way for the development of optical biosensing devices able to detect the presence of certain molecules at extremely low concentrations. Among them, photonic integrated structures are finding a great success due to their considerably high sensitivity. However, the sensing mechanism of these structures is based on the interaction between the evanescent wave, propagating along the structure surface, and the target analyte to detect. In this way, not all the field propagating in the photonic structure is used for sensing purposes, but rather only a small amount of it. This represents a crucial limitation of the integrated photonics based sensors.

The aim of this $\mathrm{PhD}$ Thesis is to overcome this limitation and to develop more sensitive photonic sensing structures able to detect the lowest concentration possible. To this aim, we focused on the study of porous silicon as platform for the development of optical structures with extremely high sensitivities thanks to the fact that the sensing interaction takes place directly inside the structure itself, allowing to exploit all the field propagating in the structure. 



\section{Resumen}

La salud y el bienestar siempre han sido el centro de atención de muchas instituciones de investigación y empresas de todo el mundo. Esto llevó a la tecnología a desarrollarse en los campos químico, biológico, médico y clínico con el objetivo de proporcionar una mejor protección al ser humano. Como consecuencia, ha surgido una competición entre el tiempo necesario para que la enfermedad progrese y el tiempo necesario para que el hombre trate dicha enfermedad. Para ganar esta competición, es necesario actuar con anticipación, cuando la enfermedad aún no está demasiado desarrollada. Esto es posible realizando una detección precoz de la enfermedad. El logro de este objetivo allana el camino para el desarrollo de dispositivos ópticos de biosensado capaces de detectar la presencia de ciertas moléculas en concentraciones extremadamente bajas. Entre ellos, las estructuras integradas fotónicas están teniendo un gran éxito debido a su considerablemente alta sensibilidad. Sin embargo, el mecanismo de detección de estas estructuras se basa en la interacción entre la onda evanescente, que se propaga a lo largo de la superficie de la estructura, y el analito a detectar. De esta forma, no todo el campo que se propaga en la estructura fotónica se usa con fines de detección, sino solo una pequeña cantidad de éste. Esto representa una limitación crucial de los sensores basados en fotónica integrada.

El objetivo de esta tesis doctoral es superar esta limitación y desarrollar estructuras fotónicas de sensado más sensibles que sean capaces de detectar las concentraciones más bajas posibles. Con este objetivo, nos centramos en el estudio del silicio poroso como plataforma para el desarrollo de estructuras ópticas con sensibilidades extremadamente altas gracias a que la interacción de sensado se realiza directamente dentro de la propia estructura, lo que permite explotar todo el campo que se propaga. 



\section{Resum}

La salut i el benestar sempre han sigut el centre d'atenció de moltes institucions de recerca i empreses de tot el món. Açò va portar a la tecnologia a desenvolupar-se en els camps químic, biològic, mèdic i clínic amb l'objectiu de proporcionar una millor protecció a l'ésser humà. Com a conseqüència, ha sorgit una competició entre el temps necessari per que la malaltia progresse i el temps necessari per que l'home tracte aquesta malaltia. Per a guanyar aquesta competició, és necessari actuar amb anticipació, quan la malaltia encara no està massa desenvolupada. Açò és possible realitzant una detecció precoç de la malaltia. L'assoliment d'aquest objectiu facilita el camí per al desenvolupament de dispositius òptics de biosensat capaços de detectar la presència de certes molècules en concentracions extremadament baixes. Entre ells, les estructures fotòniques integrades estan tenint un gran èxit a causa de la seua considerablement alta sensibilitat. No obstant açò, el mecanisme de detecció d'aquestes estructures es basa en la interacció entre l'ona evanescent, que es propaga al llarg de la superfície de l'estructura, i l'analit a detectar. D'aquesta forma, no tot el camp que es propaga en l'estructura fotònica s'usa amb finalitats de detecció, sinó solament una xicoteta quantitat d'aquest. Açò representa una limitació crucial dels sensors basats en fotònica integrada.

L'objectiu d'aquesta tesi doctoral és superar aquesta limitació i desenvolupar estructures fotòniques de sensat més sensibles que siguen capaces de detectar les concentracions més baixes possibles. Amb aquest objectiu, ens centrem en l'estudi del silici porós com a plataforma per al desenvolupament d'estructures òptiques amb sensibilitats extremadament altes gràcies a que la interacció de sensat es realitza directament dins de la pròpia estructura, el que permet explotar tot el camp que es propaga. 



\section{Index}

Acknowledgements

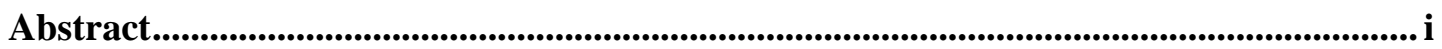

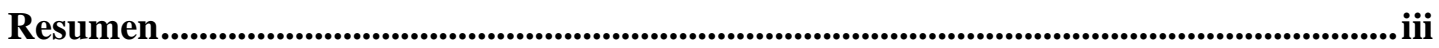

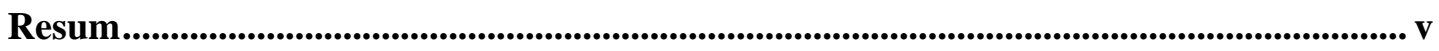

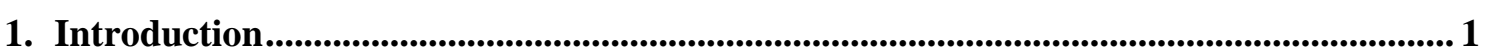

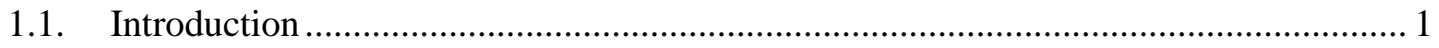

1.2. Evanescent wave mechanism based sensors .......................................................... 3

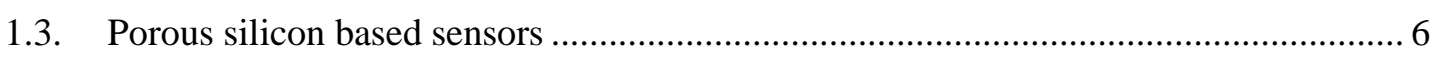

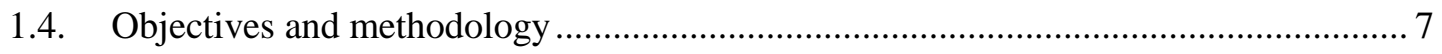

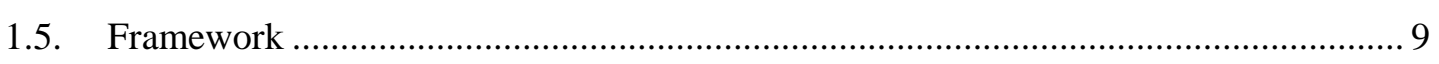

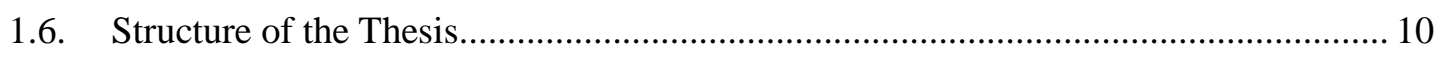

2. Optimization of antibody immobilization using protein $A$.................................................... 13

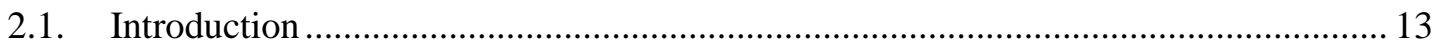

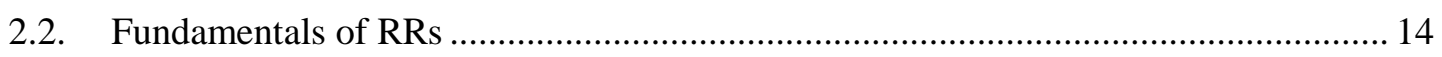

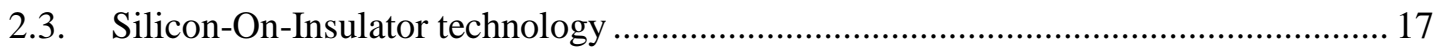

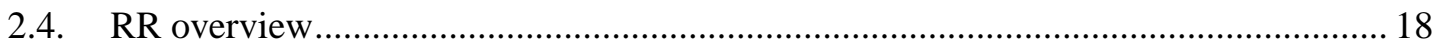

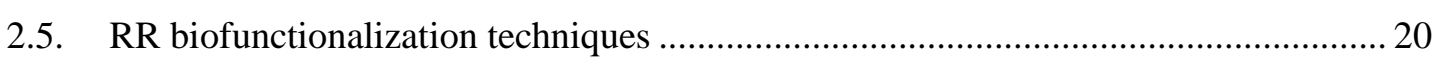

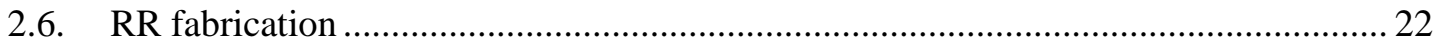

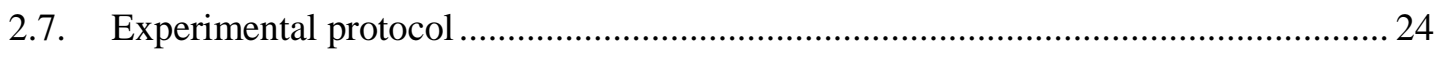

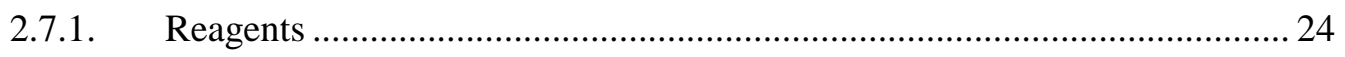

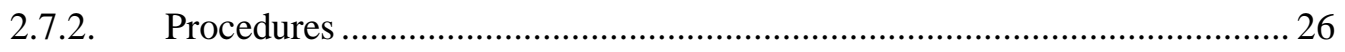




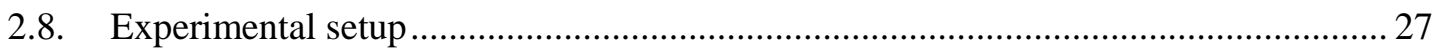

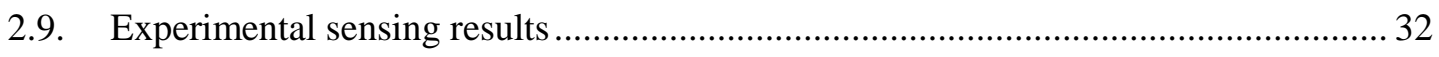

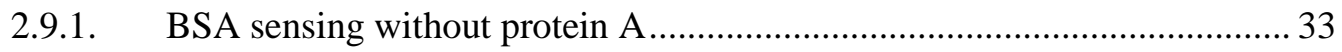

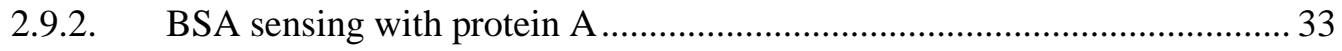

2.9.3. BSA sensing with protein A under several regeneration cycles.................. 34

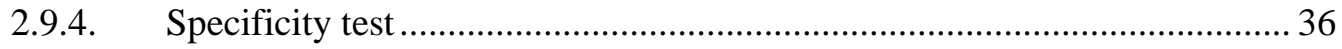

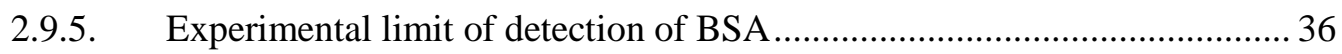

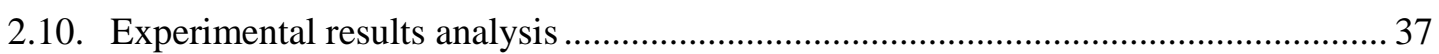

2.10.1. Effect of the protein A intermediate layer................................................... 37

2.10.2. Sensitivity for antiBSA and antiBSA detection limit................................ 38

2.10.3. BSA detection limit and specificity .......................................................... 39

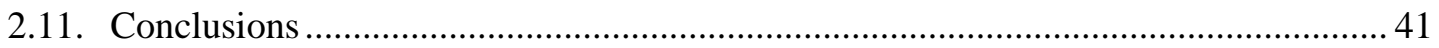

3. Sensing using porous silicon 1D periodic structure ............................................................... 43

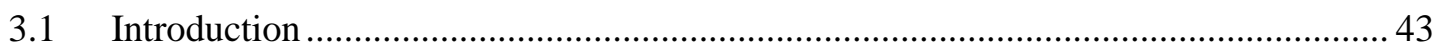

3.2 Fundamentals of PS 1D periodic structures ……....................................................... 44

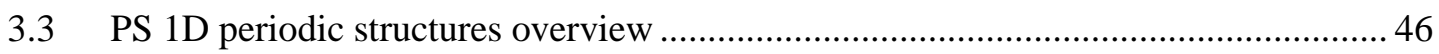

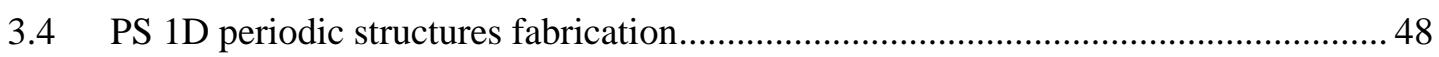

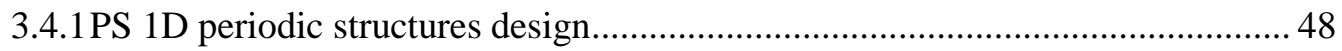

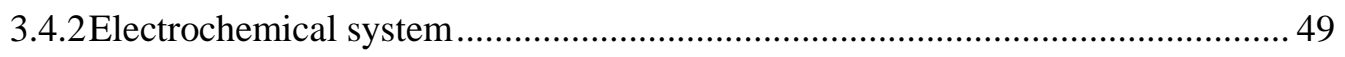

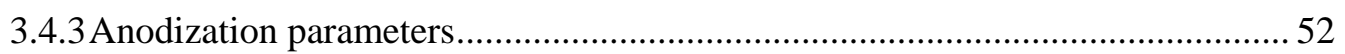

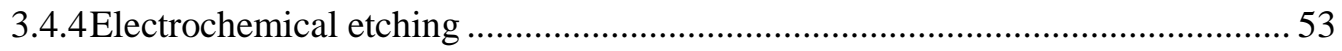

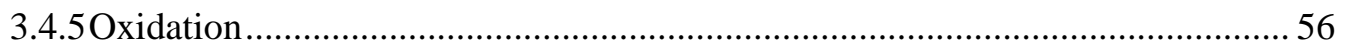

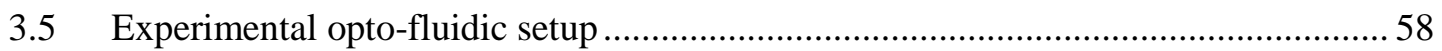

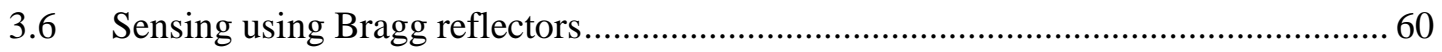

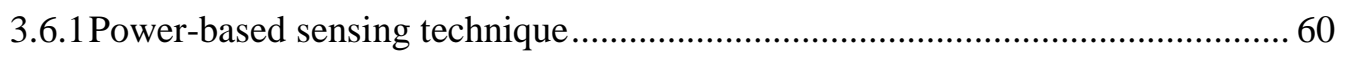

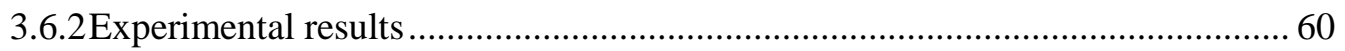

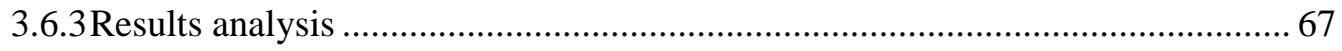

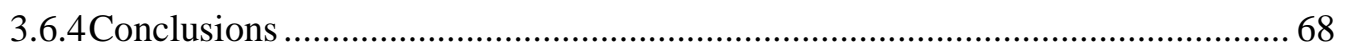

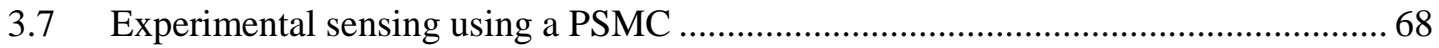




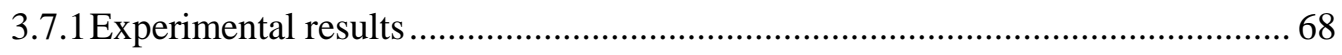

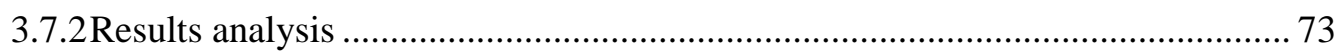

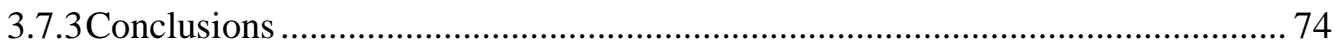

4. Temperature sensing using porous silicon fiber-optic sensing probe................................. 75

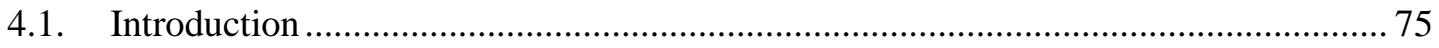

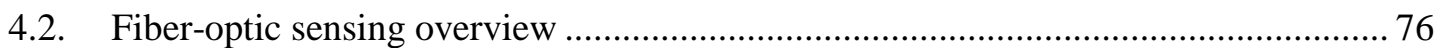

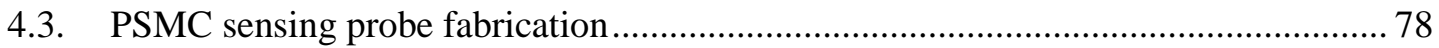

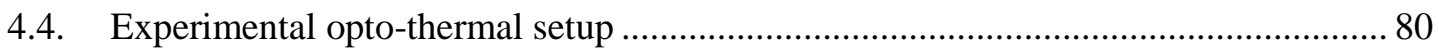

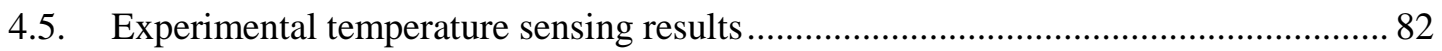

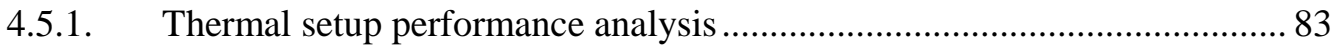

4.5.2. Water environment experiments .............................................................. 84

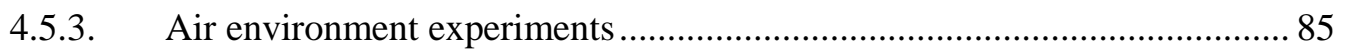

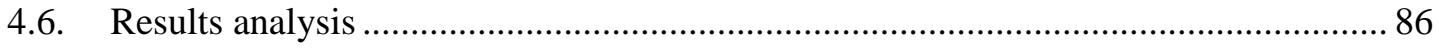

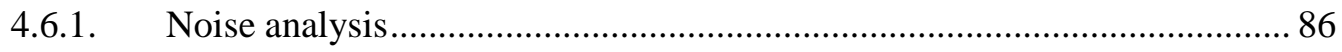

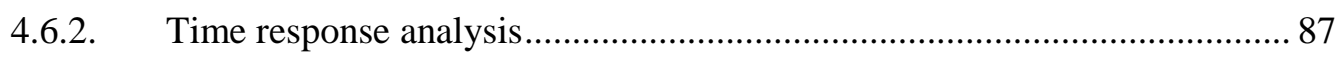

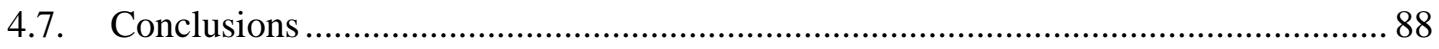

5. Experimental sensing using a porous silicon ring resonator...................................................8 89

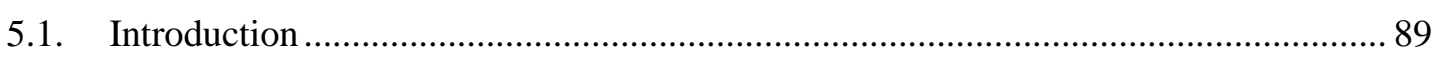

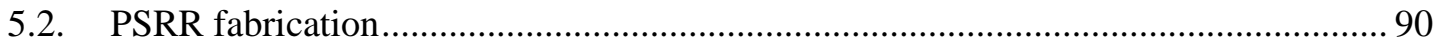

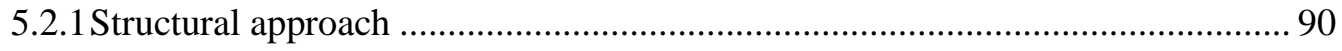

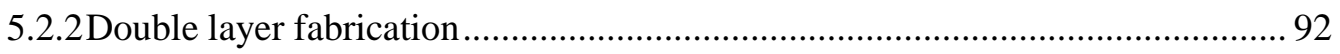

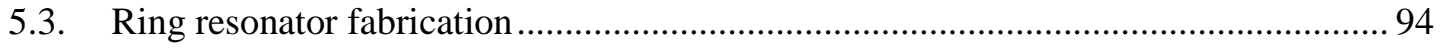

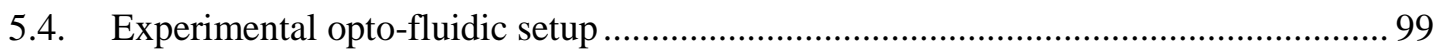

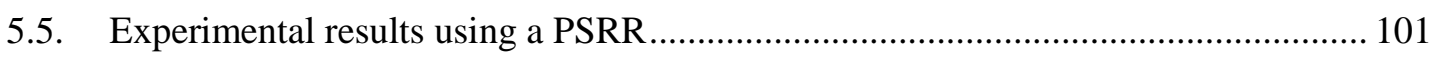

5.2.3PSRR optical characterization ...................................................................... 101

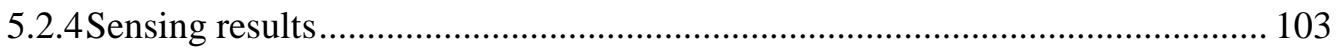

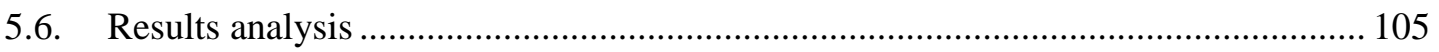

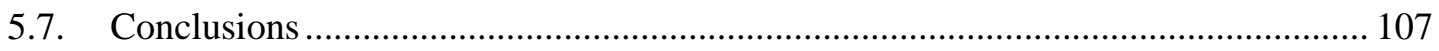




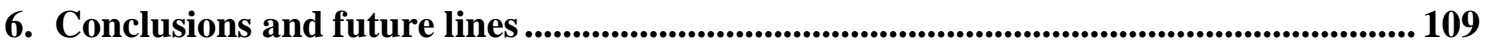

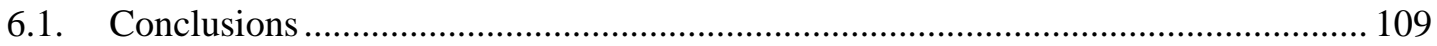

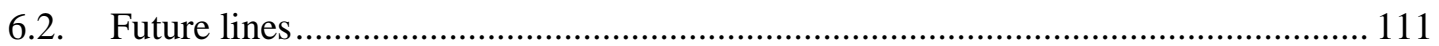

Author publications .......................................................................................................................... 113

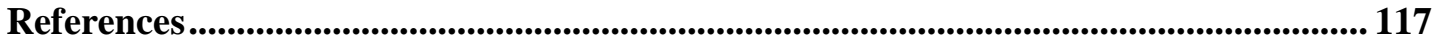




\section{Chapter 1}

\section{Introduction}

\subsection{Introduction}

Optical sensors can be defined as optical devices able to detect a variation of a certain physical quantity (e.g., refractive index, pressure, temperature) in their environment and convert it into a measurable and processable signal. Due to their extremely high sensitivity, fast response and reduced size, which permits to integrate several sensing structures on a single chip for an ultra-sensitive multiplexed detection [1-8], optical sensors have been a significant object of research and have been attracting the interest of several fields, in particular of chemical, biological and medical fields [9-14]. In fact, in the last decade, we have witnessed the rapid development and growing interest in medicine, biotechnology and drug discovery. Consequence of it, an increasing dependence on chemical analysis and a progressive demand for the rapid and precise detection have also been produced. The fulfilment of these requirements has been achieved mainly with the development of optical biosensors. Optical biosensors employ the interaction of the light with the target analyte in order to convert the biological information into a measurable signal. These sensors have a number of advantages over other transduction technologies such as 
mechanical and electrical biosensors, also due to the own nature of the optical measurements that endow a great number of different techniques, such as emission, absorption, fluorescence, refractometry, or polarimetry [15]. Furthermore, such devices allow the development of labelfree assays where the use of radioactive, colorimetric or fluorescent labels, which introduce complexity and loss of activity to biological molecules, is avoided [16-20]. Therefore, advanced label-free biosensors can provide selective, sensitive, fast and direct detection of a certain target analyte at extremely low concentrations.

It is important to consider a list of requirements in order to compare the performance of different sensors. The following list summarizes several parameters/properties that can be used to evaluate the performance of a sensor [21]:

- Sensitivity: is the relationship between the variation of the sensor output signal as a response to a physical quantity variation and the physical quantity variation itself. This parameter defines the ability of the sensor to detect that variation and to transduce it into a measurable signal.

- Resolution: is the smallest change that can be observed in the output signal. To calculate the resolution, the noise of the system must be characterized in a steady state condition without any sensing variation.

- Limit of detection (LOD) or detection limit (DL): is defined as the minimum amount of sensing information that can be detected by the sensor. This parameter is calculated considering the ratio between the noise of the measurement and the sensitivity of the sensor.

- Response time: is the time needed by a sensor to perceive the variation of a physical quantity and to react to it. This requirement may be crucial for those applications where a prompt response is needed.

- Dynamic range: is the range between the highest and the lowest values of the sensor output signal related to a sensing event. The lower limit of the dynamic range will be determined by the limit of detection of the sensor.

- Throughput or multiplexing level: is the capability of the device to carry out several parallel sensing measurements in the same test. It is crucial to obtain more information per test, to reduce the number of tests (and, thus, the cost per test) and to provide a more complete analysis. 
- Sample volume: is the amount of sample required to reliably realize a measurement. This parameter has to be carefully considered where the target sample is expensive or difficult to obtain.

- Portability: refers to the capability of the sensor to be moved in order to perform insitu measurements.

Since an optical biosensor is used for biochemical analysis, it has to be able to interact specifically with the selected biomolecules. In order to be able to interact with those biomolecules, an optical sensor must be "biofunctionalized" [22-24]. The biofunctionalization of a sensor consists in the creation of a layer comprising biomolecules apt to interact with the environment, as schematically illustrated in Fig. 1.1. This layer is also named biolayer and imputes the term "bio" to the biosensor since it is the part that provides the biological interaction. The biofunctionalization is achieved by coating, with specific techniques, the sensor surface with proper bioreceptors that can recognize the target analyte among the other particles present in the sample. This means that this biolayer provides the sensor the ability to selectively detect the presence of a certain target analyte. The selective detection of an analyte is determined by the affinity between the bioreceptors and their specific target analytes. For this reason, when the target analytes interact with the biolayer, they will be the responsible of the chemical and physical changes to be detected.

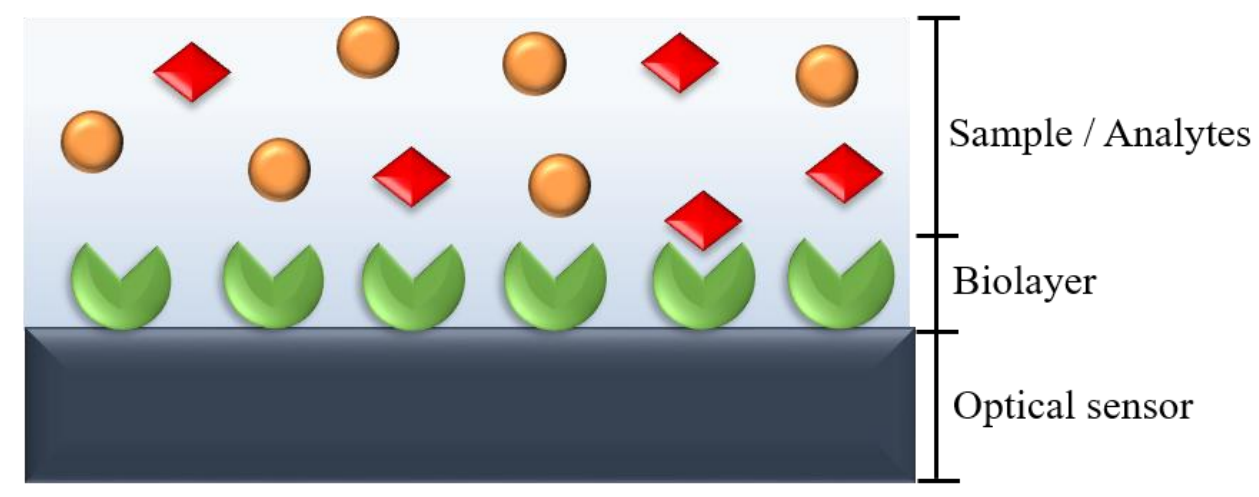

Fig. 1.1: Schematic illustration of the biofunctionalization of an optical sensor.

\subsection{Evanescent wave mechanism based sensors}

Within optical biosensors, those based on integrated photonics are probably arising a higher interest because of their outstanding characteristics of sensitivity, label-free and real-time detection, what makes them one of the main candidates to become the transduction elements of future lab-on-a-chip devices. 
In the majority of photonic biosensors, the sensing mechanism relies in the variation of the effective index of the propagating mode that occurs when a variation of the chemical or physical parameters is produced on the surface of the structure. When an optical wave is traveling through the core of an optical waveguide, a fraction of its energy propagates to the surrounding medium, as illustrated in Fig. 1.2. This fraction of energy is known as evanescent wave as it decreases its intensity exponentially from the surface.

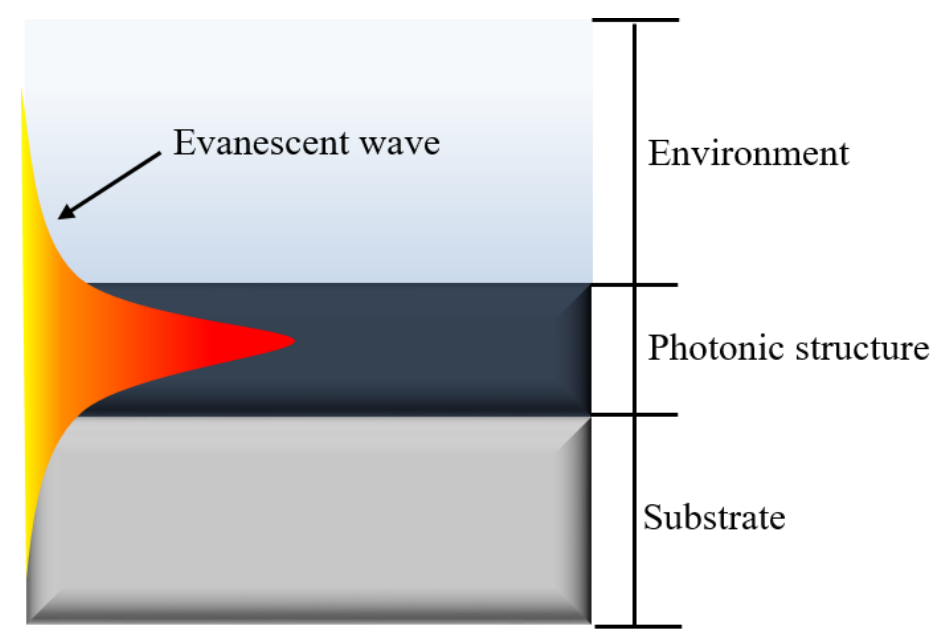

Fig. 1.2: Illustration of the evanescent wave propagation on the photonic structure surface.

The evanescent wave is the responsible of the changes in the effective index of the propagating mode when the sensing events are produced. In fact, in the evanescent wave mechanism, the exposure of the photonic sensor surface to biomolecules induces a local change of the optical guiding properties of the waveguide, as shown in Fig. 1.3. This change is detected via the evanescent wave and its amplitude can be correlated to the biomolecules concentration, yielding a quantitative signal of the interaction.

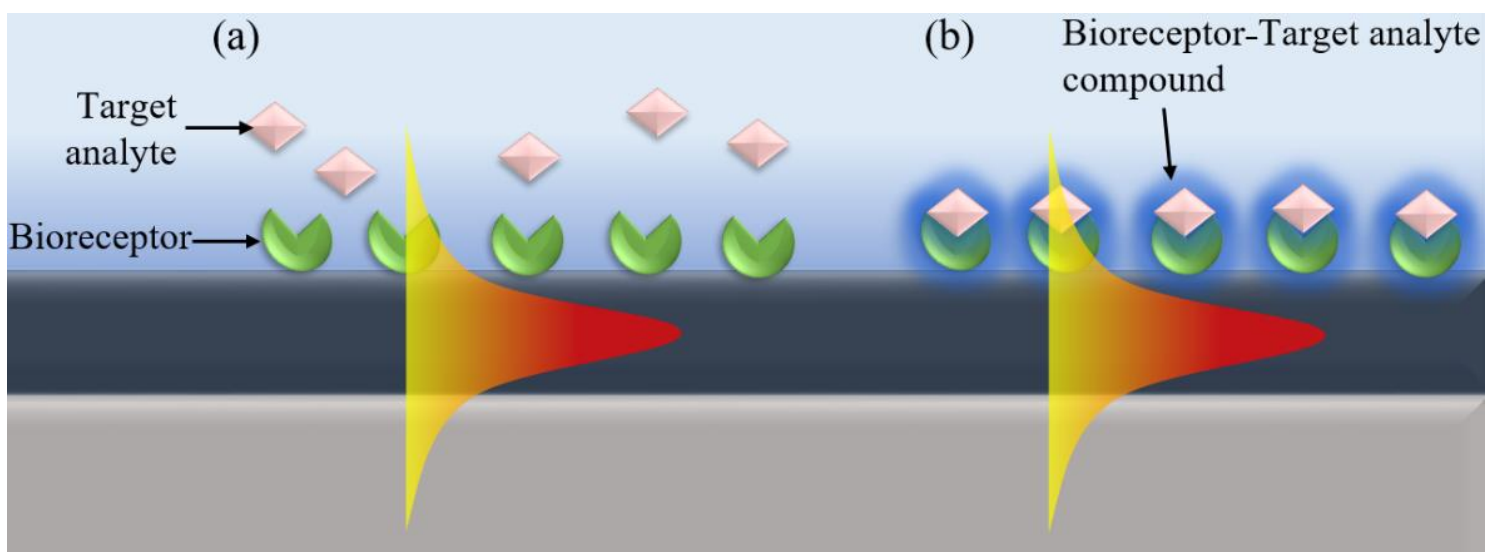

Fig. 1.3: Schematic illustration of the evanescent wave sensing mechanism: the evanescent wave propagates along the photonic structure surface and interacts with (a) the bioreceptors layer and (b) the bioreceptors-target analyte compound layer. 
It is worth noting that, as the evanescent wave decays exponentially while it penetrates into the outer medium (usually this decay length is of the order of $0.1-1 \mu \mathrm{m}$ ), it only detects changes taking place on the surface of the waveguide (or very close to it), since the intensity of the evanescent field is much higher in this particular region.

Photonic biosensors based on evanescent wave detection have demonstrated its outstanding properties, such as an extremely high sensitivity for the direct measurement of biomolecular interactions, in real time and in label-free schemes. In order to convert the effective index variations into a measurable signal such as intensity or frequency variations, different configurations of photonic structures have been developed. Among the most common configurations, we can find ring resonators [25], photonic crystals [26] and Mach Zehnder interferometers [27], schematically depicted in Fig. 1.4 (a), (b) and (c), respectively.

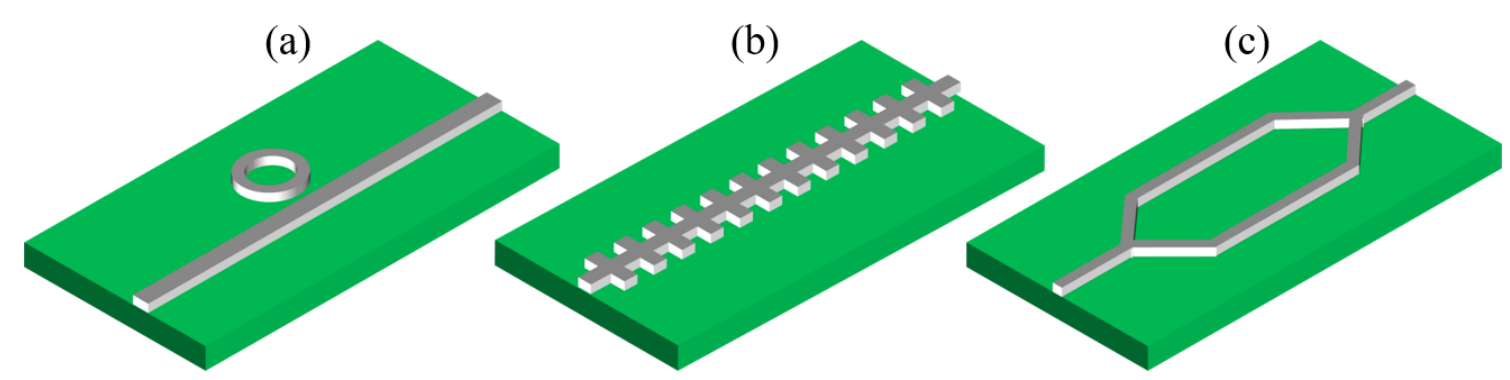

Fig. 1.4: Schematic illustration of most typical photonic sensing configurations: (a) micro-ring resonator; (b) photonic crystal; (c) Mach Zehnder interferometer.

Despite all the presented advantages, integrated photonic sensors also exhibit limitations. In a traditional planar photonic sensing structure based on the evanescent wave mechanism, only the evanescent wave propagating outside the photonic structure itself is used for sensing purposes, whereas the majority of the optical field distribution associated with the guided mode propagates within the structure itself. This fact limits considerably the performance of this kind of structure.

One way to overcome this limitation is by modifying the waveguide dimensions. As a matter of fact, it is agreed that the more amount of evanescent wave on the surface, the higher the interaction of the field with the target analyte. However, in order to achieve a high performance evanescent wave based sensor, it is necessary to reach a compromise between the portion of the evanescent field that travels on the surface and the portion that is confined on the waveguide core. An excessive amount of evanescent field would provoke a significant degradation of the performance of the guiding structure itself making the signal very weak. Therefore, the design parameters will play a crucial role in determining the performance of the sensor. 
Finally, another issue is related with the biofunctionalization of the sensor surface. As previously said, the evanescent field is characterized by an exponential decay from the surface. This causes a strong dependence of the sensitivity with the distance of the target analyte from the surface. Since the biolayer represents a new layer formed on the sensor surface, which separates the target analyte from such a surface, the interaction between the evanescent wave and the analyte is weaker, as can be appreciated in Fig. 1.5. Furthermore, some kinds of biofunctionalization techniques require several biofunctionalization steps, avoiding to obtain a biolayer as thin as required. This makes the interaction to be weaker or almost no-existent. In this way, the detection would be minimum or could even not take place.

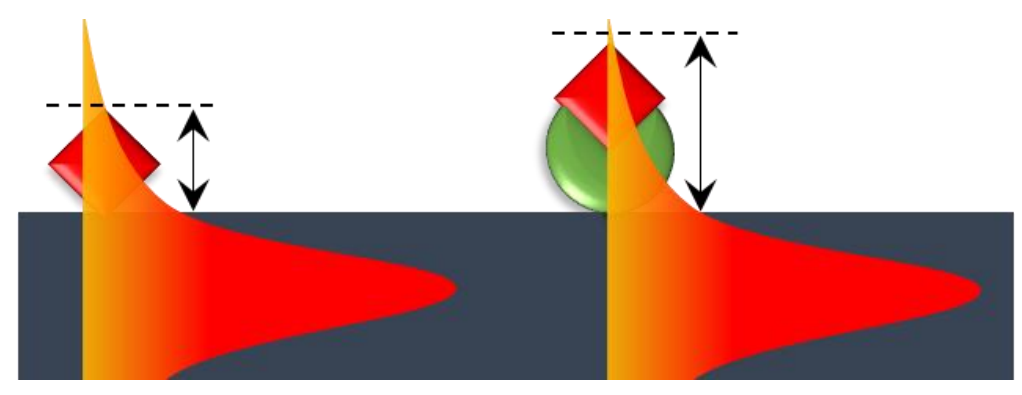

Fig. 1.5: Illustration of the interaction between the target analyte and the evanescent wave without and in presence of the biolayer. The presence of a thick biolayer leads to a weaker interaction.

In this $\mathrm{PhD}$ Thesis, we have worked on overcoming these limitations by considering an alternative platform to develop new photonic structures and exploit new detection mechanisms. This alternative platform is porous silicon.

\subsection{Porous silicon based sensors}

Porous silicon (PS) is the product of the electrochemical etching of silicon. PS came to light in 1956, at the U.S. Bell Laboratories, as an accidental result obtained by Arthur Uhlir Jr. and Ingeborg Uhliras while trying to develop a new electrochemical technique for polishing silicon surfaces [28]. Such discovery did not raise interest in the scientific world of that time, until 1990, when Leigh Canham discovered the visible luminescence properties of PS [29]. Starting from that moment, the scientific community focused the research study on the properties of such material.

With regards to optical sensing, in the last two decades, the study of PS and its applications have been increasing. Due to its several advantages, it represents a way to improve the possibilities of silicon structures and to solve their limitations. One of the main advantages of PS based optical sensors is the high sensitivity due to the fact that the sensing interaction takes place directly inside the structure itself [30]. This leads to exploit all the field propagating in the 
structure. Furthermore, due to its morphology, comparable to that of a sponge, PS presents an enormous internal surface and the PS surface can be permeated by several chemical reagents [31]. As a consequence, the high surface-to-volume ratio improves the efficiency of the surface functionalization, as it detects a higher amount of biomolecular interactions [32]. Moreover, PS can be formed simply, quickly and inexpensively since it is the result of the electrochemical etching of a silicon substrate.

Thanks to these advantages, PS has been widely used in optical sensing applications, in particular to develop sensors based on refractivity [33]. Among them, PS reflectivity based sensors are the most employed. In this kind of sensors, the sensing mechanism is based on the study of the reflectivity spectrum of the PS structure. More in detail, the refractive index change of the PS structure, due to a change of the substance filling the pores, provokes a shift of the reflectivity spectrum.

Several configurations of PS sensing structures have been reported in the last years, including single layers, as Fabry-Perot filters [34], multilayers, such as Bragg reflectors (BRs) [35] and optical microcavities (MCs) [36], which are schematically illustrated in Fig 1.6. Some of those configurations have also been implemented in a membrane format by removing the silicon substrate below the PS structure [37-40].

(a)

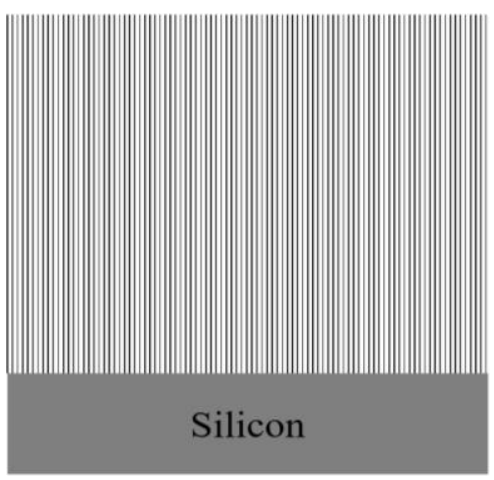

(b)

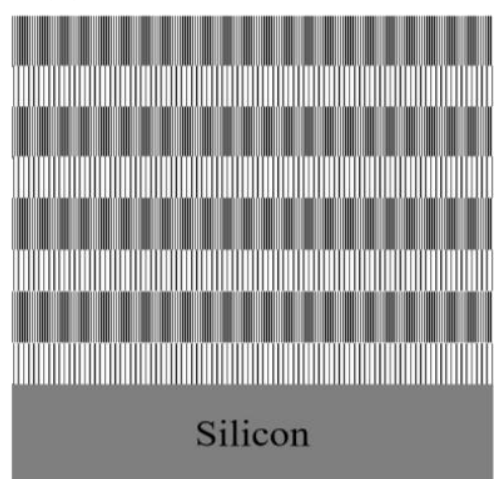

(c)

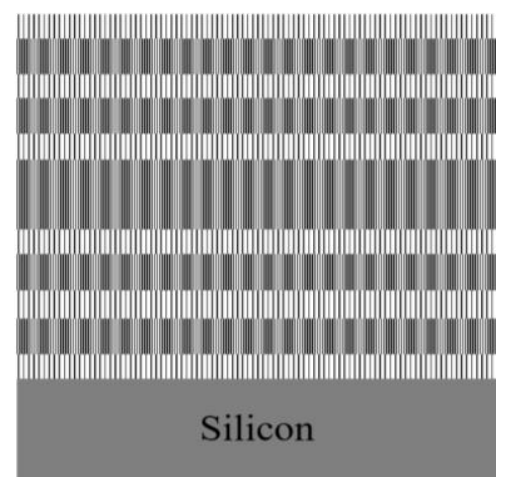

Fig. 1.6: Schematic illustration of PS optical sensing configurations: (a) Fabry-Perot filter (PS single layer); (b) Bragg reflector (PS multilayer); (c) Microcavity (PS multilayer with central defect).

\subsection{Objectives and methodology}

The overall objective of this PhD Thesis has been the development of photonic sensing configurations with a sensitivity significantly higher than that reported in the state of the art. To reach this objective, the research started with the study of traditional evanescent wave photonic structures and was redirected to new sensing PS configurations. Hence, the overall objective turned into overcoming the limitations of traditional solid core optical sensing structures by 
developing novel PS sensing configurations with higher sensitivities. Several sub-objectives had to be accomplished, including the development of an electrochemical system to prepare PS, the fabrication of the PS photonic sensing structures, the implementation of several experimental setups, and finally, the execution of many sensing experiments. Here, we summarize the main activities and the methodology employed to carry out this $\mathrm{PhD}$ Thesis:

a) Development of an efficient biofunctionalization technique: To carry out a reliable biosensor, the biofunctionalization technique needs to be as efficient as possible. In this study, the optimization of this step was achieved by performing several biosensing experiments using a traditional evanescent wave photonic structure as sensing element. The aim was to achieve the detection of bovine serum albumin (BSA) at concentrations in the range of $1-10 \mathrm{ng} / \mathrm{ml}$. These experiments performed at the initial stage of this $\mathrm{PhD}$ period determined the starting point for the development of the PS based optical sensors.

b) Understanding the formation and fabrication of PS: This objective was the base on which we built the main part of this work. The complete knowledge of the PS formation was fundamental to understand how to fabricate new PS photonic structures. For this purpose, a good collaboration with the Micro- and Nanoelectronics Department of the Belarusian State University of Informatics and Radioelectronics, in Minsk, was established, since they are experts in porous silicon formation and characterization.

c) Development of the electrochemical etching system: Once the formation of PS was clear and it was controlled how to fabricate it, the task to face up was the building up of the only missing component for the fabrication of PS structure, which was the electrochemical etching system. The accomplishment of this objective implied a study regarding the configurations used nowadays, the purchases of all the component of the system and, finally, the development and testing of several prototypes until the achievement of the definitive one.

d) Development of the highly sensitive PS photonic sensors: This objective implied the execution of several tasks simultaneously. First of all, a simulation process was needed in order to understand how the structures had to be realized. Then, also the configuration of the anodization parameters had to be setup. Once these tasks were completed, the optimal fabrication of the PS structure was achieved. The aim consisted in the achievement of several configurations of PS layers, as monolayers, double layers and multilayers, with a high homogeneity, a pore diameter in the range of 10-15 nm, in order to enhance the surface-volume ratio as much as possible, and, finally, with a porosity as high as possible in order to obtain a higher light-matter interaction. 
e) Development of the experimental setups: Once the PS photonic structures were fabricated and characterized, sensing experiments were needed in order to characterize the performance of the designed structures. To this aim, one experimental setup per type of photonic structure was implemented. This objective required a considerable effort. As a matter of fact, all the photonic structures presented a different configuration and consequently required a specific optical setup.

f) Development of experimental sensing measurements and validation of the sensing platforms: After the development of all the elements separately in the previous points (PS photonic sensors and experimental setups), the final objective of this $\mathrm{PhD}$ Thesis was the combination of all these parts in order to perform the validation of the whole system. The final of aim was the achievement of sensitivity values remarkably higher than those provided by traditional sensing configurations, up to $1000 \mathrm{~nm} / \mathrm{RIU}$ (Refractive Index Unit).

As it will be described in this Thesis, all these objectives have been successfully accomplished, what has also led to the publication of the achieved results in several journal papers and to the acceptance of contributions in several national and international conferences.

\subsection{Framework}

This $\mathrm{PhD}$ Thesis has been developed over a period of four years at the Nanophotonics Technology Center (NTC) of the Universitat Politècnica de València, within the frame of the Biophotonics research group. During the course of this $\mathrm{PhD}$ Thesis, the author was granted with a Santiago Grisolia grant from the Generalitat Valenciana.

The research developed in this period was mainly related with the development of the photonic sensing structures and with the study of porous silicon, but it also included other very important fields and activities such as microfluidics, biofunctionalization and signal processing.

An important component of the $\mathrm{PhD}$ research was working in several National and European projects related to the development of photonic biosensors. This led to work together with several National and European researchers, providing a wide panorama from which learning and also new stimuli to keep the research on. Those projects listed are here below:

a) European Projects:

- BELERA (Reinforcing carbon nanotubes and photonics research cooperation between the Belarusian State University of Informatics and Radioelectronics and the European Research Area). 
- SAPHELY (Self-amplified photonic biosensing platform for microRNA-based early diagnosis of diseases).

- PHOCNOSIS (Advanced nanophotonic point-of-care analysis device for fast and early diagnosis of cardiovascular diseases).

b) National Projects:

- BIOGATE (Ultrasensitive nanophotonic biosensors based on chain reaction using nanoscopic molecular gates).

- OPTONANOSENS (Detection of toxins and pathogenic agents using nanometric optical biosensors for CBRN threats).

\subsection{Structure of the Thesis}

The path travelled in this Thesis begins with the study of the biofunctionalization of traditional evanescent wave photonic sensors and its optimization. Once this process is optimized, we focus on the enhancement of the sensitivity of photonic structures. For this reason, we move to the study of porous silicon and its properties, in order to use such platform for the fabrication of highly sensitive optical sensors. Once the use of porous silicon for the improvement of refractive index sensing is validated, we confirm its suitability also for the direct measurement of physical parameters. Finally, the goal of this path is the development of a porous silicon integrated photonic sensor.

This Thesis document is organized in 6 chapters. The first chapter aims to introduce the philosophy of our approach, which consisted in identifying the limitations of traditional photonic sensors based on the evanescent wave mechanism and proposing porous silicon as an alternative platform for solving such limitations.

Chapter 2 presents the study and the optimization of the immobilization of antibodies on the surface of a traditional planar ring resonator using an intermediate protein A layer. This approach proved to be simple, fast and cheap, thanks to the possibility to immobilize the antibody directly in flow, and efficient, as the presence of protein A allowed the correct antibody orientation, leading to the detection of low concentrations of the target analyte. In fact, thanks to this strategy, bovine serum albumin was detected at considerably low concentrations of down to $1 \mathrm{ng} / \mathrm{ml}$.

Chapter 3 contains the experimental fabrication process of porous silicon onedimension periodic structures and their use for refractive index sensing. Bragg reflectors and optical microcavities were used as photonic sensors and extremely low refractive index variations 
were detected. In particular, refractive index variations around $6 \cdot 10^{-3}$ were detected and a sensitivity of $1000 \mathrm{~nm} /$ RIU were achieved using an optical microcavity. These results demonstrated that porous silicon is a suitable candidate for the development of new integrated photonic sensing structures.

Chapter 4 is dedicated to the use of porous silicon for monitoring physical parameters. A novel porous silicon based fiber-optic temperature sensing configuration was developed by combining an optical fiber with a porous silicon microcavity as sensing element. This approach led to an extremely high temperature resolution, in the range of $10^{-4}{ }^{\circ} \mathrm{C}$.

Chapter 5 presents the final aim of this $\mathrm{PhD}$ Thesis: the development of a highly sensitive porous silicon ring resonator and the experimental study of its performance. A sensitivity of $439 \mathrm{~nm} / \mathrm{RIU}$ was obtained, which means an enhancement of the sensitivity 4 fold over a traditional planar ring resonators. The photonic structure exhibited a higher sensitivity in TE polarization due to the higher light confinement inside the structure, which led to a higher lightmatter interaction.

Chapter 6 provides a discussion regarding the conclusions and the future lines. 


\section{Chapter 2}

\section{Optimization of antibody}

\section{immobilization using protein $\mathrm{A}$}

\subsection{Introduction}

In this chapter, we have focused the attention on the optimization of the immobilization of antibodies on a photonic sensing structure. To this aim, we studied a bio-functionalization approach based on the use of an intermediate layer of protein A able to properly orient the antibody receptors. To validate this technique, we considered a well-known antibody-antigen complex usually used as model in experimental developments: Bovine serum album (BSA) and anti-Bovine serum album (antiBSA). Several immobilization protocols were studied in order to optimize the antibody immobilization. To develop the sensing experiments, among the several integrated photonics based sensing configurations, we selected the ring resonator (RR) as photonic sensing structure.

The chapter is organized as follow. In section 2.2, the working principle of the RR, as well as its main parameters, and the sensing mechanism of this structure are explained. Afterwards, in section 2.3, we introduced the Silicon-On-Insulator technology used for the 
development of integrated photonic structures. In section 2.4, an overview of some of the more relevant works related to sensing experiments using this sensor configuration is provided. In section 2.5, the most common immobilization techniques used with RRs are reported. In section 2.6, the design and the fabrication of the RR-based sensing structures used in this $\mathrm{PhD}$ work are described. Section 2.7 deals with the experimental protocol, which means the reagents and the procedure followed to flow them during the experiments. The experimental set-up developed to continuously characterize the RR-based sensing structures and to flow all the reagents over the sensor surface is presented in section 2.8. Finally, in section 2.9, we present the experimental sensing results obtained making use of these RR-based photonic sensors and, in section 2.10, their analysis and discussion, which allowed us to obtain the conclusions presented in section 2.11.

\subsection{Fundamentals of RRs}

A basic configuration of the RR, which is shown in Fig. 2.1, consists of a close bend waveguide that confines the signal and supports circulating waveguide modes, and that is coupled to one or two straight waveguides (the case of coupling to two access waveguides is shown in the figure). Once light is coupled, it propagates through the ring in the form of whispering gallery modes. In this way a constructive interference is generated in the multiple roundtrips over the ring.

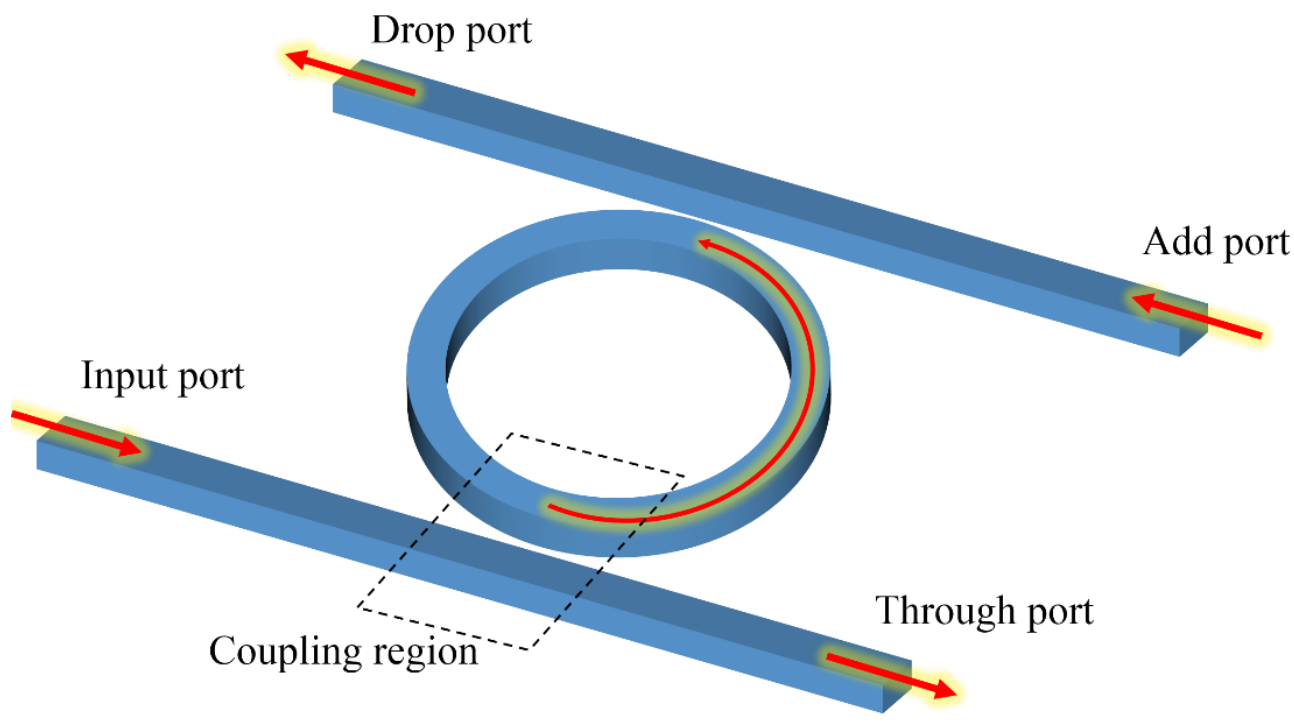

Fig. 2.1: Schematic view of a RR closely coupled to two access waveguides.

Light is coupled to the RR through the coupling region. When the phase of the roundtrip along the loop is a multiple of $2 \pi$, the wave interference that is obtained is constructive and the resonant mode is excited. The resonance mode wavelength is estimated by using the following equation: 


$$
\lambda_{\text {res }}=\frac{\mathrm{n}_{\text {eff }} \cdot \mathrm{L}}{\mathrm{m}},
$$

where $\mathrm{L}$ is the physical length of the $\mathrm{RR}$ (which is calculated as $\mathrm{L}=2 \pi \mathrm{r}$ for a perfectly circular $\mathrm{RR}$ of radius $r$ ), $n_{\text {eff }}$ is the effective refractive index of the structure, and $m$ is an integer.

Fig. 2.2 shows a common spectrum at the output of the through and the drop ports for a RR coupled to two access waveguide.

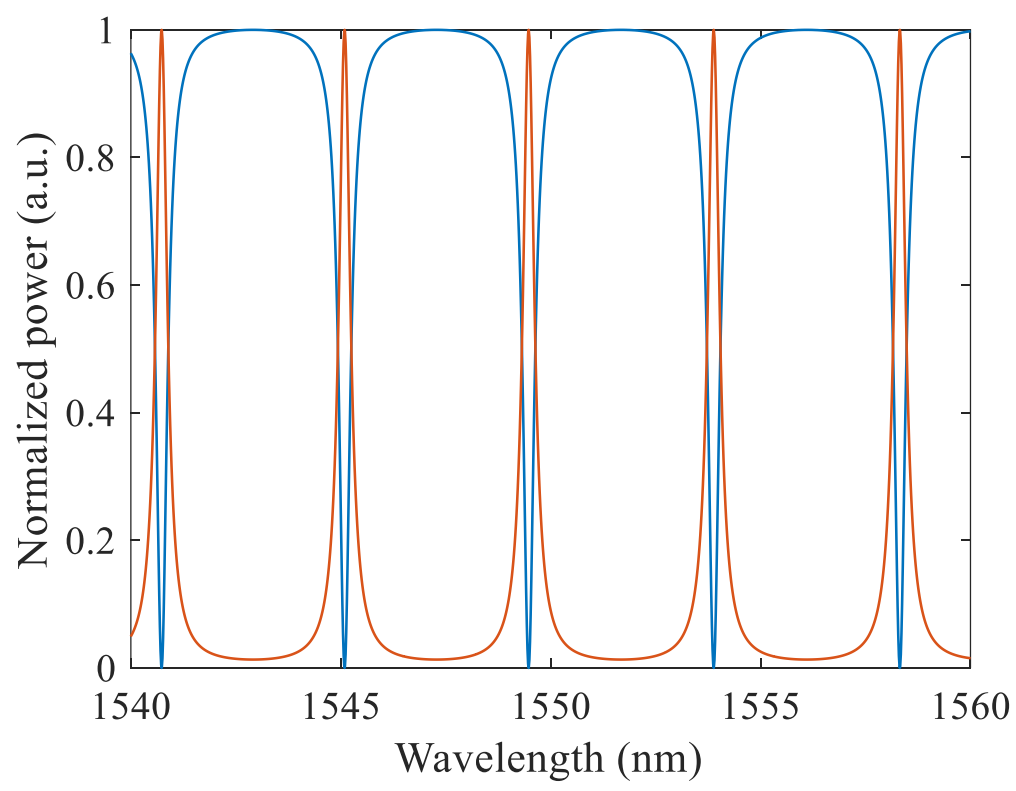

Fig. 2.2: Typical output spectra of a RR in add-drop configuration. It is characterized for exhibiting periodic dips in the through port (in blue) and peaks in the drop port (in red) resulting from the RR resonances.

One of the most important RR parameters that characterize the spectral response is the distance between two consecutive resonances, what is called free spectral range (FSR). We can obtain an approximation of the FSR by using the propagation constant of the mode and ignoring the wavelength dependence due to the dispersion of the material, thus obtaining:

$$
\mathrm{FSR}=\Delta \lambda_{\text {res }} \approx \frac{\lambda^{2}}{\mathrm{n}_{\mathrm{g}} \cdot \mathrm{L}}
$$

where $\mathrm{n}_{\mathrm{g}}$ is the group index and it is defined by

$$
\mathrm{n}_{\mathrm{g}}=\mathrm{n}_{\text {eff }}-\lambda_{0} \frac{\mathrm{dn}_{\text {eff }}}{\mathrm{d} \lambda}
$$

Another parameter related to the spectral response of the RR deals with the resonance peak width. The full width at half maximum (FWHM) is the width of the resonance dip when the power decreases $3 \mathrm{~dB}$. 
One of the most important parameters in a RR is the quality factor $(\mathrm{Q})$ and it represents the resonance sharpness. The $\mathrm{Q}$ factor is defined as:

$$
\mathrm{Q}=\frac{\lambda_{\text {res }}}{\Delta \lambda_{\text {FWHM }}} .
$$

For the case of using a RR for biosensing purposes, $Q$ factor will determine the level of interaction of the field with the target analytes, since it will determine the number of times that the light "sees" the analytes. This effect leads to a larger effective light-analyte interaction length, which can be calculated by:

$$
\mathrm{L}_{\mathrm{eff}}=\Gamma \frac{\mathrm{Q} \lambda}{2 \pi \mathrm{n}_{\mathrm{eff}}}
$$

where $\Gamma$ is the factor related to the amount of interaction between the light and matter in the cavity and the other parameters were defined previously. Good quality RRs may exhibit Q factors usually in the range from $10^{4}$ to $10^{8}$. Therefore, a RR with a physical length of tens of microns will exhibit an effective interaction length of tens of centimeters. Additionally, a high Q-factor also means having very sharp resonances, whose position can be more accurately determined when carrying a sensing experiment out..

The detection employing RRs is based on tracking the shift in the position of the resonances, which are induced by the refractive index variation provoked by the binding of the target analyte, as schematically shown in Fig. 2.3. Initially, the RR guiding structure is characterized by an effective refractive index $n_{\text {eff, }, 1}$, which corresponds to a resonance mode wavelength $\lambda_{\text {eff, }, 1}$, represented in red. When the target analytes reach the RR, the effective refractive index changes to a new value $n_{\text {eff, }, 2}$, provoking a displacement of the resonance to $\lambda_{\text {eff, }, 2}$, represented in blue, and a total spectral shift of $\Delta \lambda$. In this way, by analyzing the wavelength shift it is possible to immediately detect and quantify the presence of target analytes, even if the analyte concentration is low.

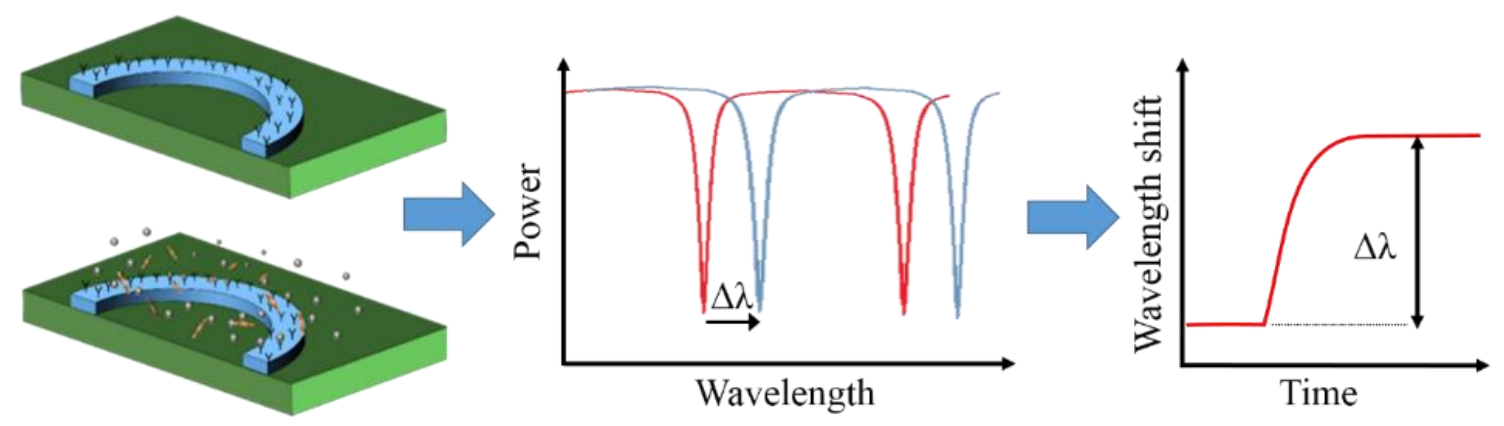

Fig. 2.3: Schematic illustration of the working principle of an optical RR-based biosensor. When the target analytes reach the RR surface, the resonances will shift to higher wavelengths. 


\subsection{Silicon-On-Insulator technology}

Silicon (and its derivatives) is the most widely employed material for the fabrication of integrated photonic structures due to its compatibility with CMOS (Complementary Metal Oxide Semiconductor) fabrication processes from the microelectronics industry. This led to the creation of the so-called silicon photonics technology platform. In fiber optic telecommunication applications, the silicon is used for the development microphotonic components operating in the infrared, usually at the $1550 \mathrm{~nm}$ wavelength. The most common implementation of silicon photonics is SOI (Silicon-On-Insulator) technology. A SOI wafer mainly consists of a thin layer of silicon (of few hundreds of $\mathrm{nm}$ ) which is on top of a thicker layer of silicon oxide (of several microns), as depicted in the Fig. 2.4. These two layers are created over a thick silicon substrate (around $700 \mu \mathrm{m}$ ) that provides mechanical stability.

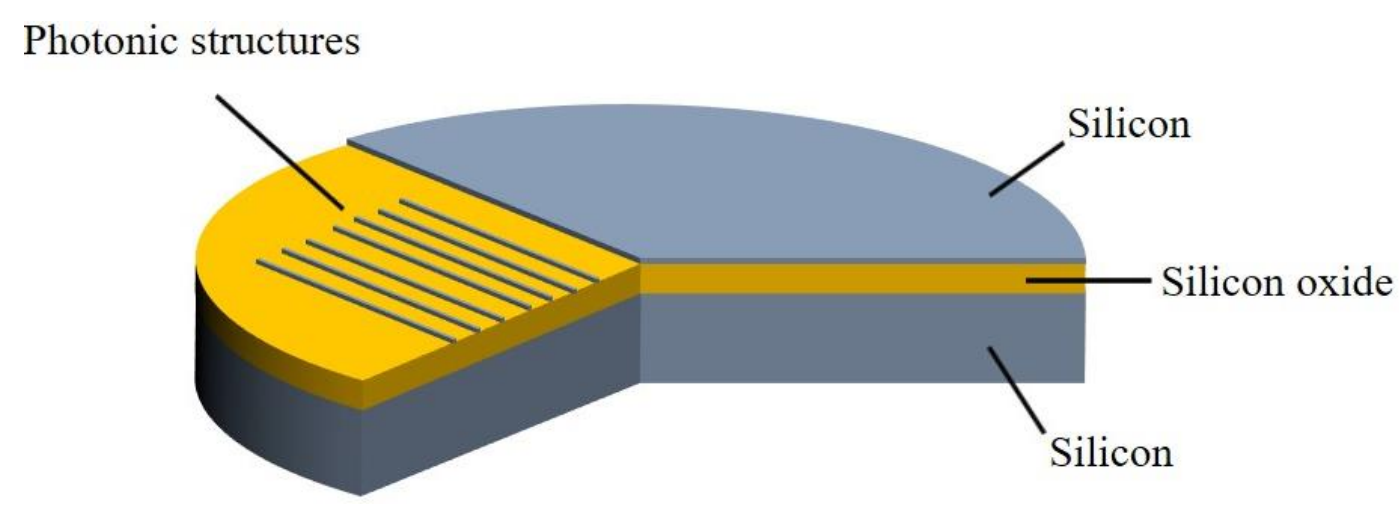

Fig. 2.4: Layers distribution of a SOI wafer.

The reason why SOI technology is largely employed for the development of photonic structures is the fact that, in SOI structures, light is highly confined in the vertical direction within the top silicon layer, which is used to create the photonic structures, as schematically illustrated in Fig. 2.5. The high light confinement is due to the high refractive index difference between the top silicon layer, the silicon oxide lower-cladding and the air upper-cladding ( 3.45 vs. 1.45 vs. 1). In order to obtain a single mode wire waveguide, the size of the cross section must be of the order of $\left(\lambda / n_{\text {core }}\right)^{2}$, where $\lambda$ is the wavelength of the light in vacuum and $n_{\text {core }}$ is the waveguide core RI. This type of waveguides with a high RI contrast allow a high confinement of the light, but they might be also sensitive to the roughness, what can lead to high radiation losses. 


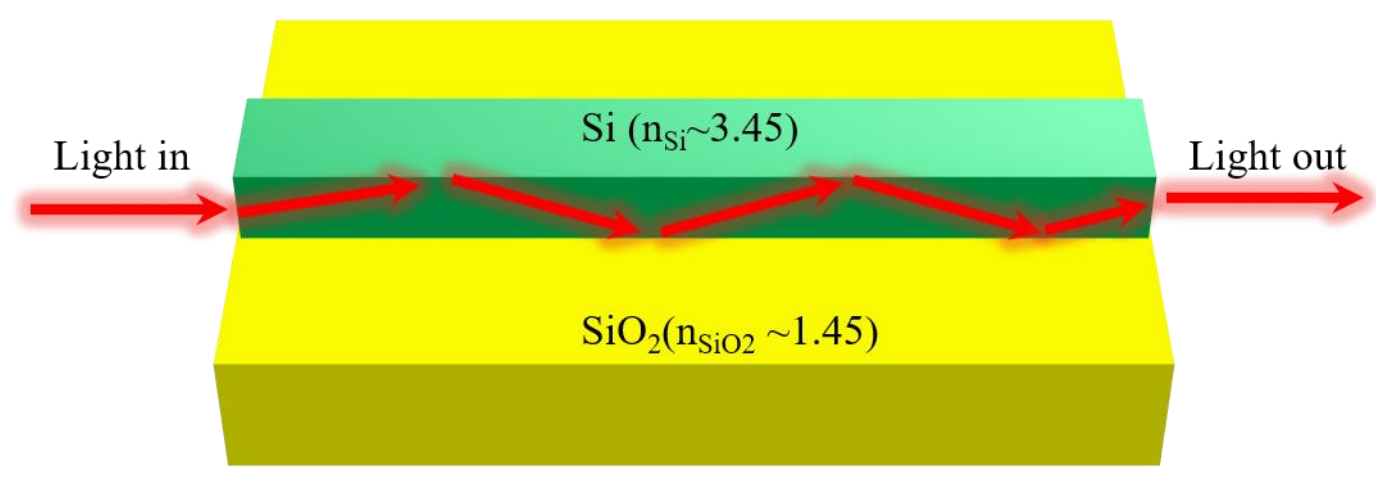

Fig. 2.5: Schematic illustration of the light confinement in a SOI waveguide, due to the high RI contrast.

One of the advantages of SOI photonic structures is the reduction of size. The size reduction leads to the possibility of integrating a large number of sensing structures in a single chip, thus permitting the possibility to provide multiplexed detection for the simultaneous screening of several target analytes in a single analysis. In this way, devices based on photonic sensors offer the possibility of integrate multiple passive and active structures in a chip.

The SOI structure size reduction leads also to an enhancement of the sensitivity of the sensing structures itself. As a matter of fact, the smaller the sensing structure is, the less amount of molecules will be required to carry the detection out. Other advantages are the relatively reduced cost and fabrication easiness of mass-manufacturing CMOS technology. The combination of all advantages provided by SOI structures leads to a significant reduction of the cost per chip and per assay. The fabrication of inexpensive sensing chips is highly required in clinical, medical and environmental control applications.

\subsection{RR overview}

RRs are being developed since some years ago in the field of integrated optics, where they have immediately found the opportunity to be implemented in different applications. Nowadays, RRs mainly find application in the field optical networks and signal processing, but during the last few years, they have also been used as sensors due to their high sensitivity and their potential to be produced in arrays for multiplexed analysis. They are characterized by a high sensitivity and, for this reason, these photonic structures are nowadays largely used to develop highly sensitivity photonic biosensors. As a matter of fact, a ring resonator is characterized by the capability to measure variations of the refractive index and, consequently, to quantify this variation by converting it into a variation of the optical signal.

The versatility of RR-based biosensors has been extensively manifested in numerous works in which such structures have been employed for sensing in gas or liquid environment. These structures are characterized by their high $\mathrm{Q}$ factor and the possibility of use different cavity 
geometries. Here, we report some possible configurations for the implementation of RRs for sensing purposes, according to their geometries, materials and coupling section.

Several waveguide cross sections can be used for the implementation of RR devices. The three most important ones are rib, wire and slot waveguides, which are schematically depicted in Fig. 2.6.

- Wire waveguide: Due to its simplicity and good performance in all the materials, this structure is the most employed. The cross section is given by a rectangular waveguide of a high index material placed on the top of a low index material (at least, with a higher refractive index than the cladding in order to confine the optical field). For the case when a high contrast configuration is used (as for the case of SOI technology), light will be highly confined inside the waveguide core, what permits to tailor structures with small bending radius and fabricate ultra-compact designs with reduced small footprints (usually in the order of $100 \mu \mathrm{m}^{2}$ ).

- Rib waveguide: This configuration allows confining the electromagnetic field within the core of the waveguide in a better way. The consequence of this better confinement is a lower light-matter interaction, what is not desirable from a biochemical detection point of view.

- Slot waveguide: This kind of waveguide has the particularity of not confining the light in the high index material, but in a low index region that can be air or even the liquid/gas sample to be analysed. Due to this capability to confine the light in the low index region, slot waveguide exhibits a very high sensitivity and it has been used in photonic sensing in a large way.

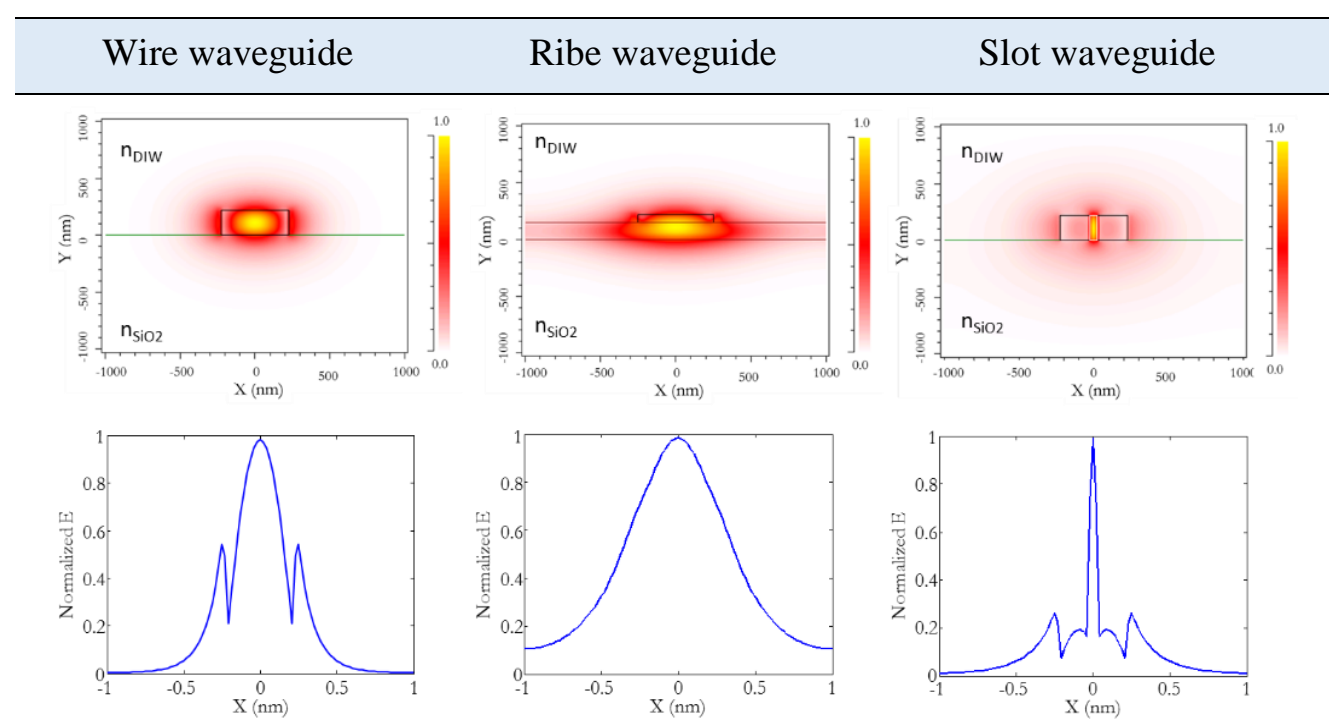

Fig. 2.6: Different waveguide architectures according to the cross section profile. 
In 2007, one of the first works regarding biosensing using a SOI wire-based RR was reported by De Vos et al. [41]. In this work, a sensitivity of $70 \mathrm{~nm} / \mathrm{RIU}$ and a detection limit of $10^{-5}$ RIU were obtained. Furthermore, a concentration as low as $10 \mathrm{ng} / \mathrm{ml}$ of avidin using biotin receptors was achieved. Even though this sensitivity was comparable with that obtained for other planar photonic structures commonly used for biosensing purposes, the difference consisted in the extremely small footprint of the RR $\left(\sim 100 \mu \mathrm{m}^{2}\right)$, which led to an outstanding theoretical mass detection limit of $1 \mathrm{fg}$. This confirmed the huge potential of RRs for the development of high sensitivity photonic sensors. In 2010, Iqbal et al. reported the highest sensitivity value for a planar RR [42]. A sensitivity of $160 \mathrm{~nm} / \mathrm{RIU}$ was obtained, almost 2 fold to the average sensitivity value of $80-100 \mathrm{~nm} / \mathrm{RIU}$ of a traditional planar RR. In 2013, Passaro et al. reported theoretical results of the sensitivity of the three RR configurations and theoretically demonstrated that slot waveguides result in a higher performance [43]. As a matter of fact, Claes et al. presented experimental biosensing results using a slot waveguide based RR in silicon technology, showing an extremely high sensitivity of $298 \mathrm{~nm} / \mathrm{RIU}$ and a LOD of $4.2 \times 10^{-5}$ RIU [44]. More recently, novel RR configurations have proposed to enhance the base sensitivity of RRs, on ultra-thin SOI substrates [45] or on sub-wavelength waveguides (SWG) [46], as shown in Fig. 2.7.
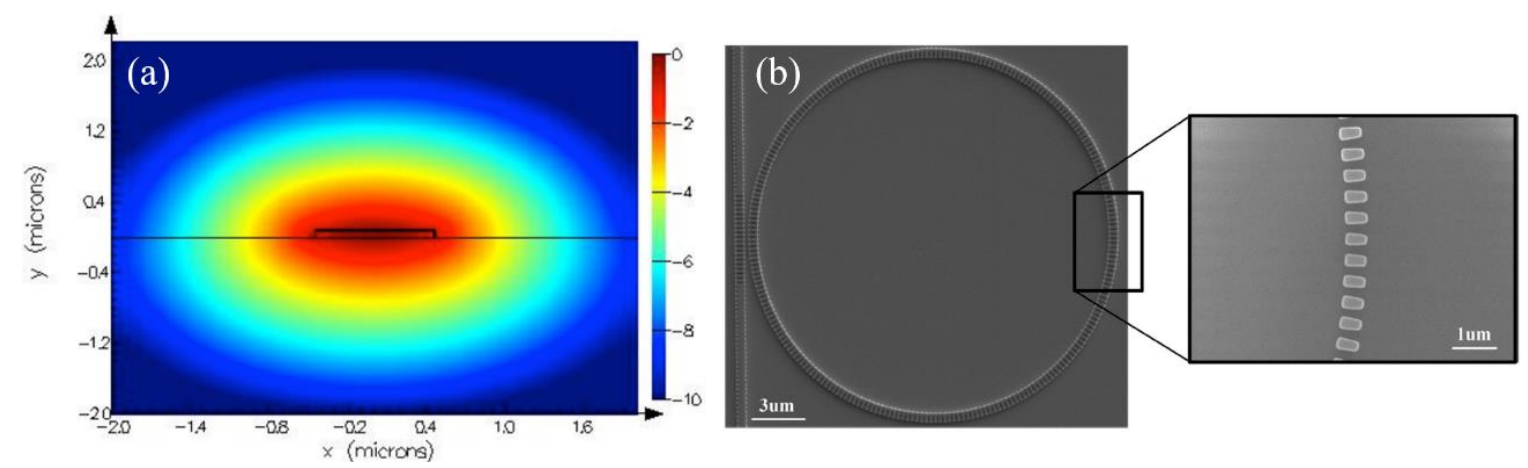

Fig. 2.7: (a) Simulation results of the cross-section of the ultra-thin silicon waveguides [45]. (b) SEM image of SWG ring resonator [46].

\subsection{RR biofunctionalization techniques}

Even though RR allows to measure variations of the refractive index, it is not characterized by the attribute to specifically recognize the presence of a certain biological molecule. As explained in chapter 1, to confer this specificity to a sensing structure, its surface must be coated with a biological layer of bioreceptors able to identify the target analytes. Accordingly, determining the optimal surface modification, which means finding a suitable coating for the immobilization of the receptors onto the surface, is crucial for the development of a sensitive and selective RR based biosensor and influences the final performance of the device. 
Among the several types of bioreceptors employed for the biofunctionalization of RR sensors, antibodies are the most used, since they provide high specificity and affinity [47, 48]. One of the most common techniques employed to immobilize antibodies on the sensor surface is the direct physical adsorption, due to its simplicity and rapidity. However, a problem can introduce a loss of sensitivity of the sensor: the random orientation of the bioreceptors when they are directly immobilized on the sensing structure. This problem can provoke that the binding between the bioreceptors and the target analytes does not take place or takes place in a wrong way, lowering the binding capacity in comparison to that in solution. Nowadays, in order to overcome this problem and obtain a highly-sensitive structure, other immobilization techniques to create a robust biolayer over the sensor surface have been developed including monolayers and covalent binding [47].

Covalent binding is one of the most used immobilization techniques, due to the strong attachment of receptors to the photonic structure surface [48]. Very low detection limits have been achieved using this technique. For example, $\mathrm{Xu}$ et al. in [49] achieved a resolution of $40 \mathrm{ag}$ of total mass. De Vos et al. in [50] achieved a limit of detection of $3.4 \mathrm{pm} / \mathrm{mm}^{2}$, with a theoretical minimum detectable total mass of 74 ag. In [51], Luchansky et al. reported a limit of detection of $1.5 \mathrm{pg} / \mathrm{mm}^{2}$. However, the protocol required to apply this immobilization technique is significantly more complex to that reported in this $\mathrm{PhD}$ work. In addition to covalent binding, other techniques have been employed for the immobilization of oriented antibodies on ring resonators. For example, Taniguchi et al. used a silicon-binding protein based technique providing a sensitivity for biomaterial of $10 \mathrm{ng} / \mathrm{mL}$ [52]. Apart from the antibody immobilization, regarding the detection of BSA, Shan et al. reported a technique based on an organophosphate surface coating and vinyl sulfone linker for the biofunctionalization of ring resonators for biomolecular sensing and detected BSA with a concentration of $250 \mathrm{ng} / \mathrm{mL}$ [53]. However, these techniques also require several steps, leading to large process times and more sample manipulation.

The approach presented in this chapter is an efficient alternative to these techniques as it allows to overcome all their technical issues. Firstly, because the proper antibody orientation provided by the presence of the protein A layer is translated into a better sensitivity of the photonic structure. Secondly, because the protein A layer can be created directly on flow. Therefore, the advantage to flow all the reagents of the whole experiment (from the biofunctionalization to the detection) avoids all the issues related with the covalent binding. 


\subsection{RR fabrication}

The chips used in the experiments were designed by the Universitat Politècnica de València and fabricated in CEA-LETI (Grenoble, France) in the frame of the cost-share European nanophotonic fabrication platform ePIXfab. The photonic structure fabricated for our experiments was a SOI RR in add-drop configuration and were fabricated employing $200 \mathrm{~mm}$ SOI wafers with a silicon thickness of $220 \mathrm{~nm}$ on top of a buried silicon oxide layer of $2 \mu \mathrm{m}$.

The fabrication process consisted of several steps, as schematically depicted in Fig. 2.8. First, a thin photoresist layer was coated on the SOI wafer by spin coating and then was prebaked. Then, a $193 \mathrm{~nm}$ Deep UV lithography was applied. In this process, the photoresist was, first, covered with a mask, where the photonic structures are designed, and then, exposed to UV light. In this way, during the exposition, the chip design patterned in the mask was projected directly to the photoresist by means of UV light. Afterwards, the wafer was placed in a post-exposure bake and was developed. In this process, the remaining photoresist solidified, becoming more resistant. In order to transfer the pattern to the silicon layer, a dry etching was carried out. In this process, the remaining photoresist acted as a mask, protecting the covered silicon, while the uncovered part was etched. Finally, the photoresist was removed and the wafer was cleaned. In Fig. 2.9, the fabricated SOI RR is shown. The basic physical parameters of the RR are reported in Table 2.1.

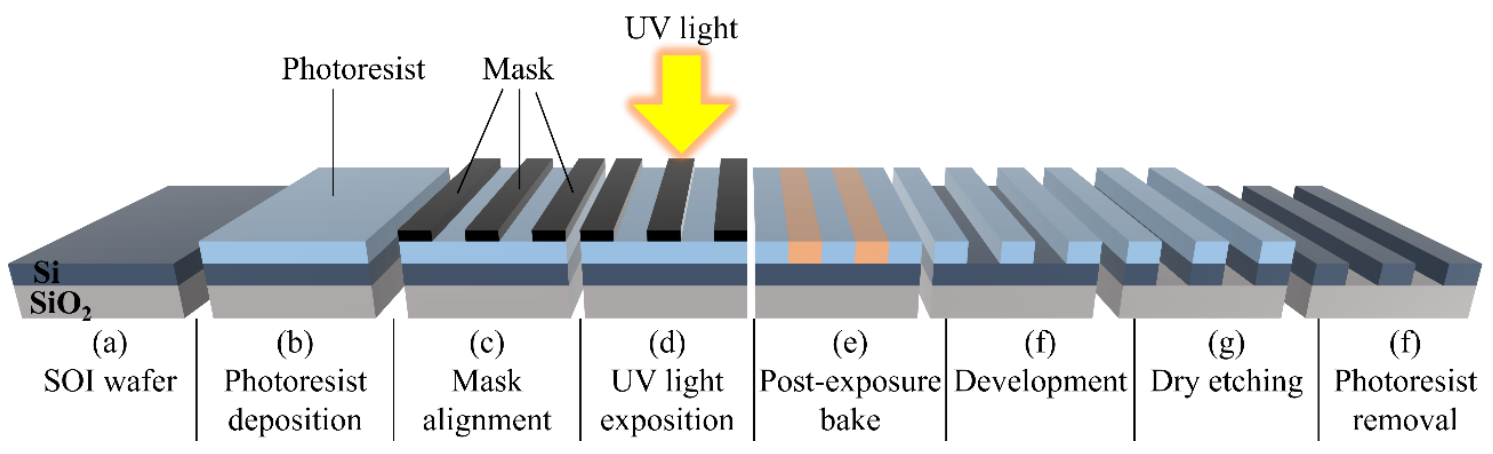

Fig. 2.8: Schematic illustration of the photonic structure fabrication.
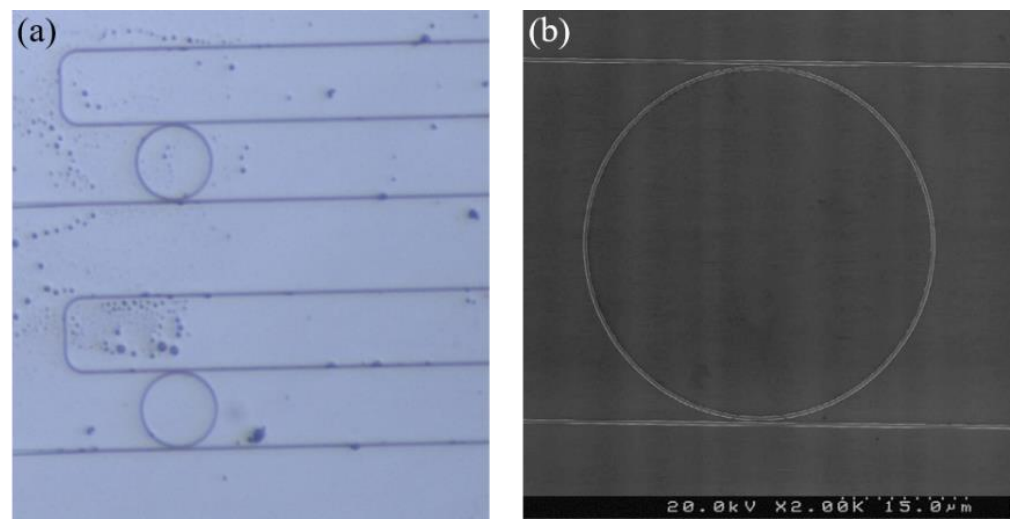

Fig. 2.9: (a) Optical microscope image and (b) SEM images of the fabricated RR. 


\begin{tabular}{cc}
\hline RR physical parameters & Dimensions \\
\hline Radius & $20 \mu \mathrm{m}$ \\
Waveguide Height & $220 \mu \mathrm{m}$ \\
Waveguide width & $450 \mu \mathrm{m}$ \\
Gap & $175 \mu \mathrm{m}$ \\
\hline
\end{tabular}

Table 2.1: Dimensions of the circular RR designed at UPV and fabricated at CEALETI.

In order to allow the light coupling, grating couplers were used to inject and to collect the light to/from waveguides. A grating coupler is a structure consisting of a periodic modulation of the refractive index at the waveguide direction. When the light beam illuminates the grating at a certain angle of incidence, an intensity exchange is produced between the mode coming from the fiber to the guided mode in the optical waveguide (or vice versa). Grating couplers are characterized for their selectivity in polarization; due to the elevated birefringence of high index contrast 1D grating structures, the so-called Bragg condition is different for TE and TM polarizations. In our designs, we have considered TE polarization and therefore the gratings have been optimized for it.

Figure. 2.10 shows a schematic illustration and an optical microscope image of the vertical coupling method to couple light by using grating couplers. Although they are polarization dependent, the alignment tolerance that exhibit is superior to horizontal coupling, what allows coupling a considerable amount of power to perform the measurements with a high alignment tolerance. Grating-based coupling methods are widely used for the optical coupling in integrated photonic devices [54].

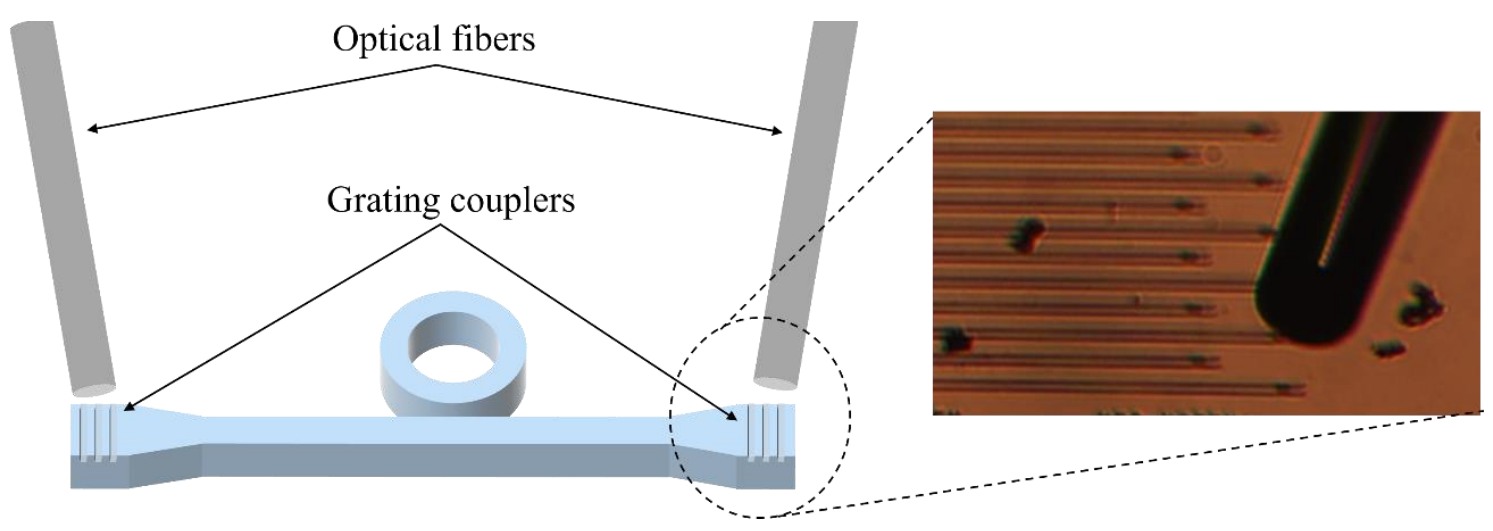

Fig. 2.10: Schematic illustration of the coupling to the photonic chip using grating couplers. In the inset, an optical microscope image of a fiber placed over a grating coupler at a distance of few $\mu \mathrm{m}$ is shown. 


\subsection{Experimental protocol}

\subsubsection{Reagents}

\subsubsection{Protein A}

As previously introduced, the bio-functionalization approach to be used is based on the immobilization of the antibodies on the sensor surface through an additional intermediate layer of protein A, so that the antibodies can be anchored to the sensor surface by binding with this intermediate layer, as it is schematically depicted in Fig. 2.11. Protein A is a 64 kDa microbial surface protein derived from the cell wall of Staphylococcus aureus [55]. It is an Fc-receptor biomolecule since it contains four binding sites specific to the Fc region of antibodies. Therefore, it specifically binds with the Fc region of immunoglobulin from many mammals, while leaving the Fab regions available for antigen binding. In this way, this bio-functionalization approach will allow that the anchored antibodies will be oriented in such way that the binding sites are exposed towards the sample solution containing the target analyte to be detected, thus maximizing the interaction with them [56]. Furthermore, since protein A provides four binding sites, it does not need a specific orientation to allow the oriented antibody immobilization. For this reason, the creation of the protein A layer can be achieved by physical adsorption, allowing to perform all the immobilization process in flow and, thus, monitoring it in real-time. This makes such a technique very simple and quick for the bio-functionalization of photonic structures. Additionally, once the antibodies are bound to the protein $\mathrm{A}$, it is possible to break this binding by using a certain reagent in order to bio-functionalize again with a new batch of antibodies of the same kind and repeat the experiment or to attach another type of antibodies and carry out a different experiment [57].
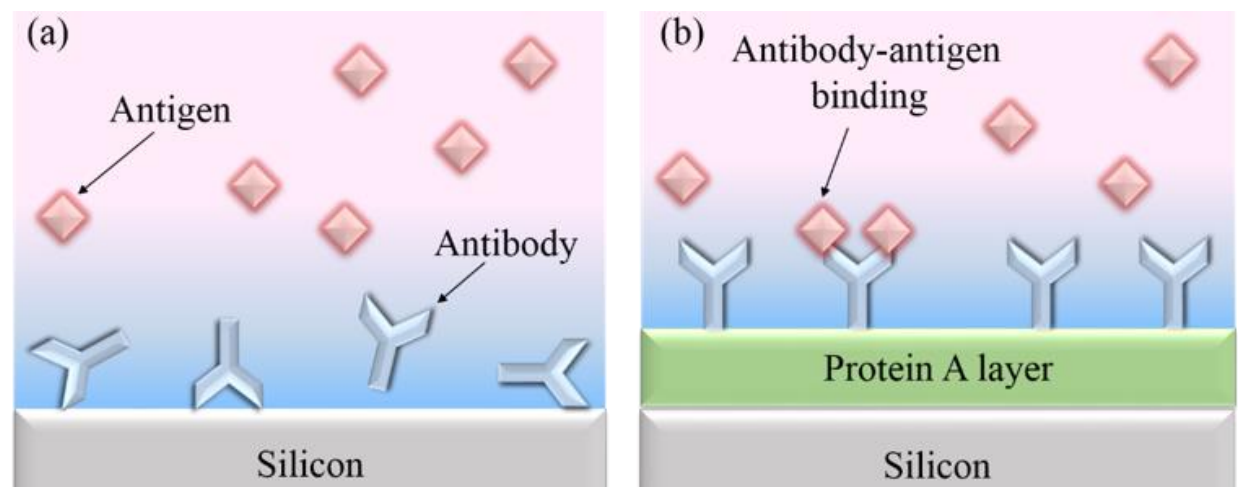

Fig. 2.11: Illustration of (a) randomly-oriented antibodies on the sensor surface directly immobilized using physical adsorption. The antibodies will not properly expose their Fab binding sites towards the sample solution with the target analytes. (b) properly-oriented antibodies on the sensor surface where an intermediate layer of protein A was used. Antibodies will bind to the protein A layer through their Fc site, thus leaving their Fab sites oriented towards the sample solution with the target analytes. 


\subsubsection{BSA and antiBSA}

The albumins are a family of globular proteins that are soluble in water and moderately soluble in concentrated salt solutions. These globular proteins are present in blood plasma. They are not glycosylated and thus are unique from the other blood proteins. The most common of the albumins is serum albumin, which is the most abundant plasma protein in mammals. Examples of serum albumin are human serum albumin (HSA) and bovine serum albumin (BSA). In the sensing experiments presented in this chapter, we used BSA as analyte model, in order to demonstrate that our photonic biosensor is able to specifically perform the detection using the developed biofunctionalization and experimental protocol. BSA is a serum albumin protein derived from cows and it is characterized by a molecular weight of $66.5 \mathrm{kDa}$.

As bioreceptor able to bind with BSA in a specific manner we utilized antiBSA, the antibody specific for this analyte. This antibody is characterized by a molecular weight of 160 $\mathrm{kDa}$, more than double of the BSA molecular weight. This means that the sensor surface will be coated by the layer of protein A and by a "thick" layer of antiBSA making the BSA detection an aim more arduous.

\subsubsection{Gelatin}

After creating the protein A intermediate layer, it is possible that not all the sensor surface is completely covered with the protein. Therefore, antiBSA can be adsorbed on the noncovered area and remain there with a random orientation. In order to block the sensor surface, Cold Water Fish Skin Gelatin (CWFS Gelatin) [58] was used. CWFS Gelatin is a gelatin of low dimensions and extremely soluble in water, which is widely employed in immunoassay because of its blocking property. Therefore, the gelatin will block the surface not covered by the protein and exposed to the flow.

\subsubsection{Glycine}

At the end of a sensing experiment, mainly three layers are created in the chip, namely a protein A intermediate layer, an antiBSA bioreceptors layer and a layer with the target BSA antigen bound to the bioreceptors. In medical and clinic fields, the assay repetitivity is a crucial test to confirm the validity of the adopted sensing procedure. In order to be able to repeat the sensor surface biofunctionalization and the BSA detection steps several times with the same photonic sensor, we broke the bond between protein A and antiBSA by flowing glycine at the end of each experiment. Glycine is the simplest amino acid and it is characterized by a single hydrogen atom as its side chain. Glycine buffer solution at $\mathrm{pH}$ 2-2.5 is a solution commonly used for eluting antibodies in various antibody affinity purification procedures, such us protein A-agarose and protein G-agarose [59]. By flowing glycine at the end of an experiment, a new experiment can be 
restarted with the same chip covered with the same protein A layer where we will bind the antiBSA bioreceptors again in order to carry out a new detection of BSA.

\subsubsection{Ovalbumin}

Finally, in order to ascertain the specific detection of BSA, we carried out also negative controls by using ovalbumin (OVA). OVA is a protein present in the egg white and it is characterized by a molecular weight of $42.7 \mathrm{kDa}$. Since OVA is not the analyte specific to the antiBSA, it does not bind with that antibody. This means that, when OVA is flowed over the sensor surface covered by antiBSA, it will not be detected and the specificity of our sensing technique can be demonstrated.

\subsubsection{Procedures}

All the experiments were carried out exclusively in flow. The standard experimental sensing procedure is shown in Fig. 2.12. The experiments began with the flow of protein A with a concentration of $10 \mu \mathrm{g} / \mathrm{ml}$ over the ring resonator sensor in order to create, by physical adsorption, the intermediate protein A layer for a better immobilization of the bioreceptors, as previously explained. PBS $0.1 \times$ (phosphate buffered saline, $0.8 \mathrm{mM}$ sodium phosphate dibasic, $0.2 \mathrm{mM}$ sodium phosphate monobasic, $13.7 \mathrm{mM}$ sodium chloride, $0.3 \mathrm{mM}$ potassium chloride, $\mathrm{pH}$ 7.5) was initially used as buffer for the protein A (as well as for all the reagents to be flowed over the sensor surface). This buffer provides a $\mathrm{pH}$ very close to that for the optimal binding of the antibody to the protein $\mathrm{A}\left(\mathrm{pH}=8.2\right.$, with high affinity $\left.\left(\mathrm{K}_{\mathrm{a}}=10^{8} / \mathrm{mol}\right)\right)$. After creating the protein A intermediate layer, gelatin at a concentration of $100 \mu \mathrm{g} / \mathrm{mL}$ in PBS $0.1 \times$ was flowed. Apart from blocking the surface against non-specific adsorption, the use of the gelatin allows us to test the proper creation of the protein A layer. If such layer is consistent and well distributed, when the gelatin is flowing, it will not fill the layer and no wavelength shift will be observed. After this point, the buffer for the experiments was changed from pure PBS to PBS with gelatin at a concentration of $10 \mu \mathrm{g} / \mathrm{mL}$, in order to keep the blocking of the surface. Then, antiBSA with a concentration of $30 \mu \mathrm{g} / \mathrm{mL}$ was flowed. Finally, in order to regenerate the sensor, at the end of every experiment, we flowed glycine, $\mathrm{pH} 2.5$, at a concentration of $10 \mathrm{mM}$ in PBS. Finally, a sandwich assay was performed after the BSA detection cycle in order to confirm that the BSA was bound to the antiBSA. It consisted on a second flow of antiBSA in order to observe an additional wavelength shift that confirms the presence of the BSA on the sensor surface. 


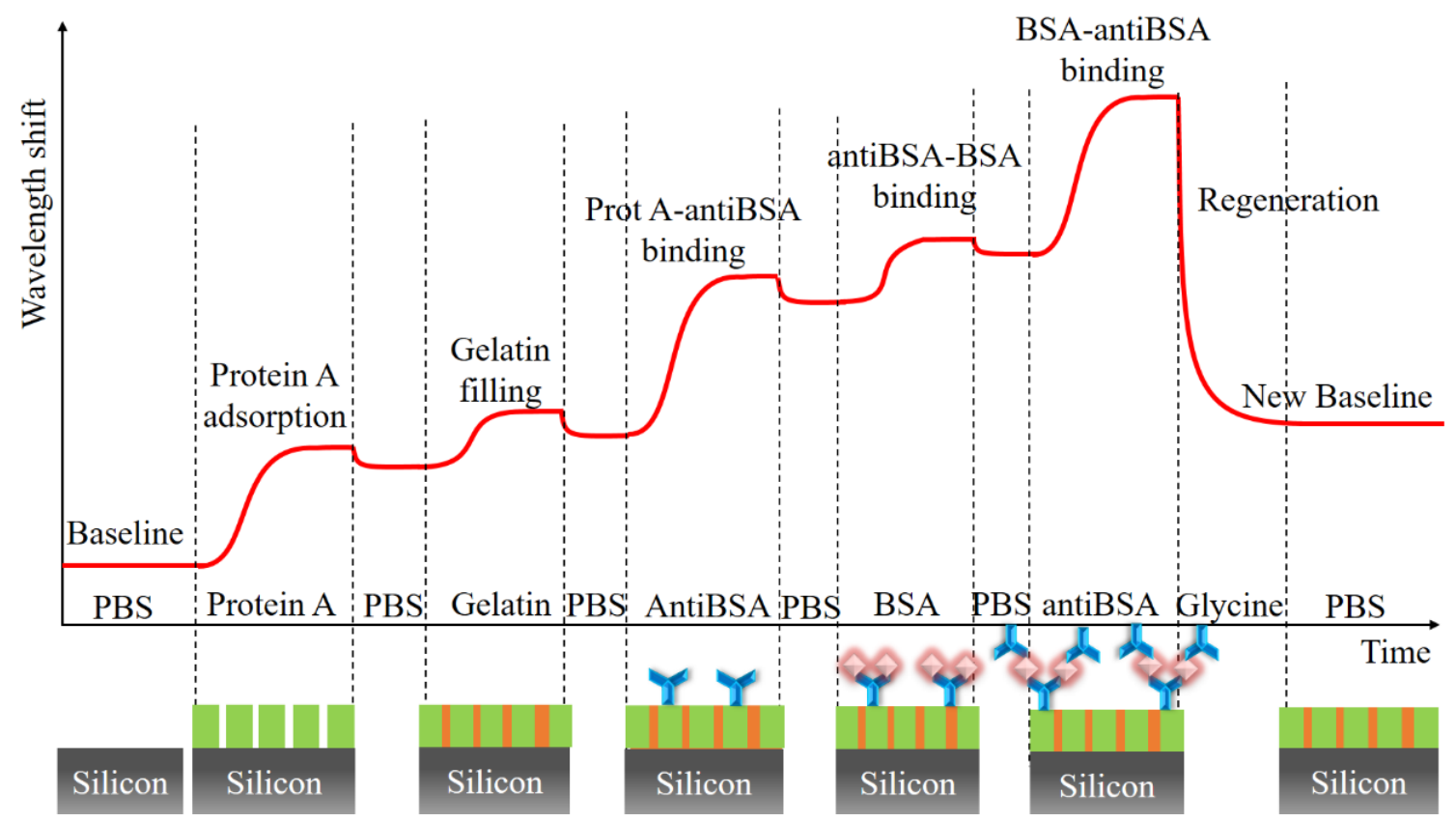

Fig. 2.12: Schematic representation of the wavelength shift evolution of the whole experiment.

In order to test the specificity of the assay, different experiments were performed adopting a second procedure. Unlike the standard procedure, after covering the RR surface with protein A and immobilizing antiBSA, OVA with a concentration of $100 \mathrm{ng} / \mathrm{ml}$ was flowed over the sensor, instead of BSA, as target analyte. Since OVA is not the specific analyte of antiBSA, they do not recognize to each other. In this way, no detection should be observed.

\subsection{Experimental setup}

A fundamental advantage of our technique consisted in flowing all the reagents over the sensor surface during the experiment in order to monitor their binding. To this aim, a system suitable for delivering the solutions with the reagents to the sensing RRs was needed. Therefore, we fabricated a basic microfluidic delivery system by using a common material employed for this type of development, the polydimethylsiloxane (PDMS). PDMS is an elastomer that presents many interesting properties for the development of microfluidic systems such as the ability to adopt the shape of the container after curing, it is a non-expensive material since it is easy to fabricate, its bio-compatibility, or that it is transparent at visible wavelengths (what makes possible to observe the evolution of the flow along the microchannel and detect any irregularity such as leakage or obstruction). Figure 2.13 shows, on the left, the fluidic channel layout and, on the right, the PDMS microfluidic channel, where two access ports are made with a hole puncher in order to insert the Teflon tubes used to pump the liquids into the microfluidic channel. The length, the height and the width of the channel were $6 \mathrm{~mm}, 50 \mu \mathrm{m}$ and $400 \mu \mathrm{m}$, respectively. 


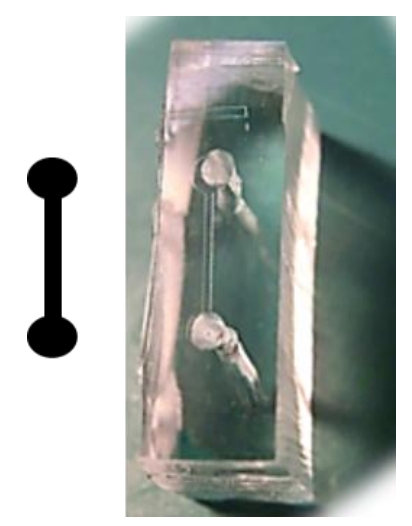

Fig. 2.13: On the left, layout of the microfluidic channel; on the right, the real implementation of this design in PDMS.

In order to properly place the PDMS microfluidic system on top of the photonic chip, with the microfluidic channel correctly aligned with the RRs of the chip, adequate equipment is required. In this case, a flip chip machine (Finetech) with an alignment tolerance of few microns was employed to accurately place the PDMS on top of the photonic integrated structures and align the microchannels with the array of sensors, as it can be seen in Fig. 2.14. Once the microfluidic channel is aligned with the RRs of the chip, some pressure is applied on the PDMS in order adhere it to the chip.

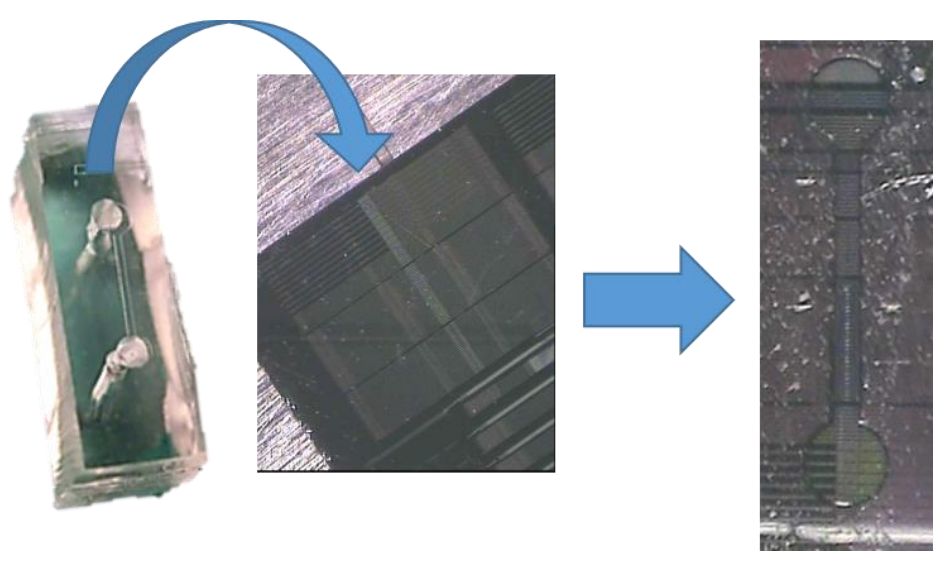

Fig. 2.14: Schematic illustration of the proper alignment of the microfluidic channel with the RRs achieved by using a Flip Chip tool. The image of the final result is shown in the right.

Once the chip-PDMS system was assembled, it was placed in the optical setup in order to continuously characterize the photonic sensing structure, as shown in Fig. 2.15. To this aim, two optical fibers were positioned on top the chip in order to couple the light through the access grating couplers, one on the side of the input waveguide and the other on the side of the output waveguide. Grating couplers used in the experiments exhibited a maximum coupling efficiency of $31 \%$ around $1550 \mathrm{~nm}$, with a bandwidth of $50 \mathrm{~nm}$, and working at an incident angle of $10^{\circ}$ respect to the vertical axis. 


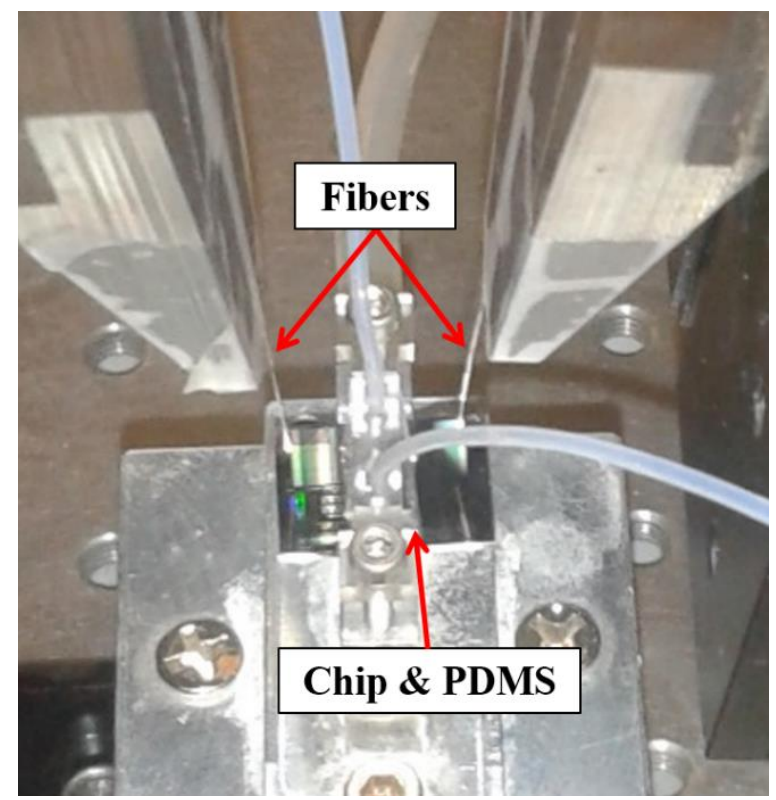

Fig. 2.15: Picture of the photonic chip-PDMS system placed on the holder of the optical setup, where the optical fibers are aligned with the input-output photonic waveguides.

Once the alignment was achieved, light from a superluminiscent diode (SLD) (SLED 8164 Covega, Thorlabs), was coupled to the chip. A SLD is a broadband source with an optical bandwidth of $50 \mathrm{~nm}$ centered in $1550 \mathrm{~nm}$. Light collected with the output fiber was divided using a fiber 10-90 directional coupler. The 10\% output port was connected to a power meter (1931-C, Newport Corporation) in order to monitor the output power during the fiber alignment process to obtain the best fiber placement. The $90 \%$ output port was connected to an optical spectrum analyser (OSA) (AQ6317C, Ando Electric Co. Ltd) in order to capture the spectrum of the photonic sensing structure.

When the biosensing experiments were carried out, the liquids were flowed with the help of a syringe pump. The pump was set to withdraw mode in order to "suck" the liquids from a vial located at the end of the microfluidic system, thus making the liquid to flow through the PDMS microchannel. Working in withdraw mode allowed changing the liquid to be flowed by simply changing the vial at the end of the system, something that is not possible when working with the pump in injection mode. The pump was set to a constant flow rate during the whole experiment. Finally, the change between the several different liquids to be flowed during the experiments was made by using a multiposition automatic switch that permits to change from 10 vessels to a common port with an electrical actuator, as shown in Fig. 2.16. The automatic switch was controlled by serial communication port using a computer. 

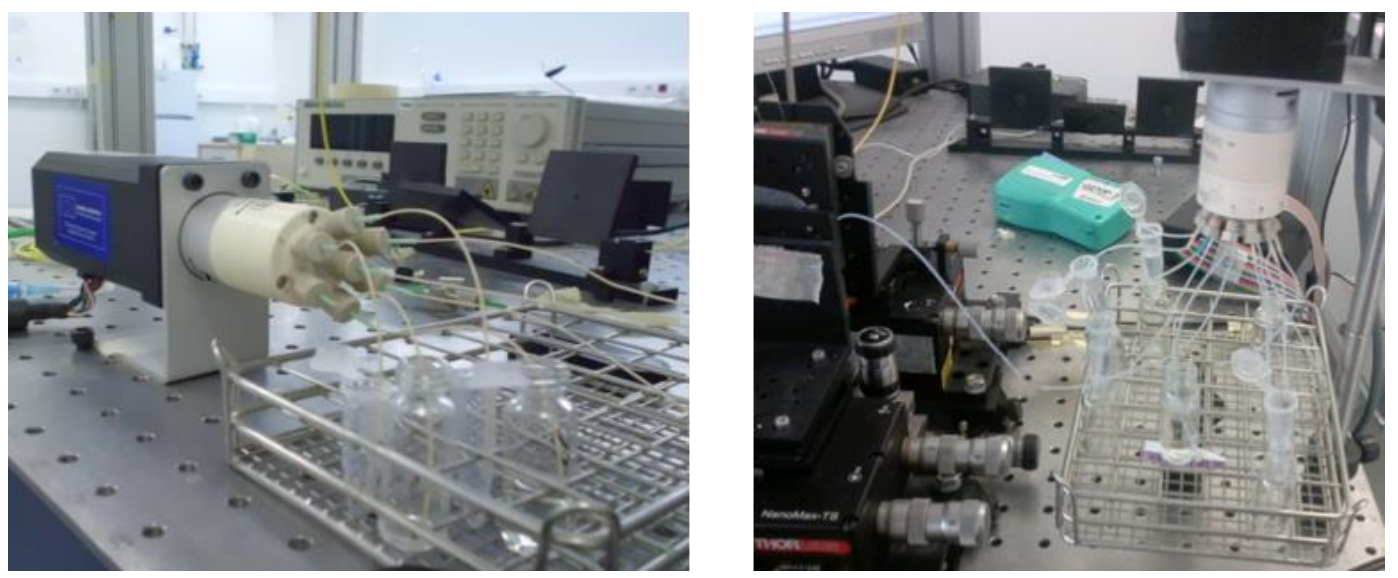

Fig. 2.16: Pictures of the automatic fluidic switch.

The whole opto-fluidic setup is shown in Fig. 2.17, whereas Fig. 2.18 shows the scheme that we used to implement this setup and to coordinate all the instruments between them. Data from the OSA were collected by means of GPIB acquisition data instrumentation and a LabVIEW customized program was used in order to continuously scan the spectrum of the biosensing device. The LabVIEW program simply receives the sweeping information from the OSA and saves it to a file in the computer, which will be later analyzed in Matlab in order to determine the evolution of the sensor's response. LabVIEW is also used to control the automated switch during the development of the experiment in order to avoid any human interference in the measurements. In this way, we can establish a pre-defined protocol where the order and the time lapse for the flow of each reagent is indicated. In our case, we usually programmed the automated switch through the LabVIEW program to flow in order protein A, gelatin, antiBSA and BSA.

In conclusion, in our experiments, the measurements are fully automatized, since the computer controls at the same time the automatic valve, the scanning of the OSA and the data acquisition of the measurement from the OSA. 


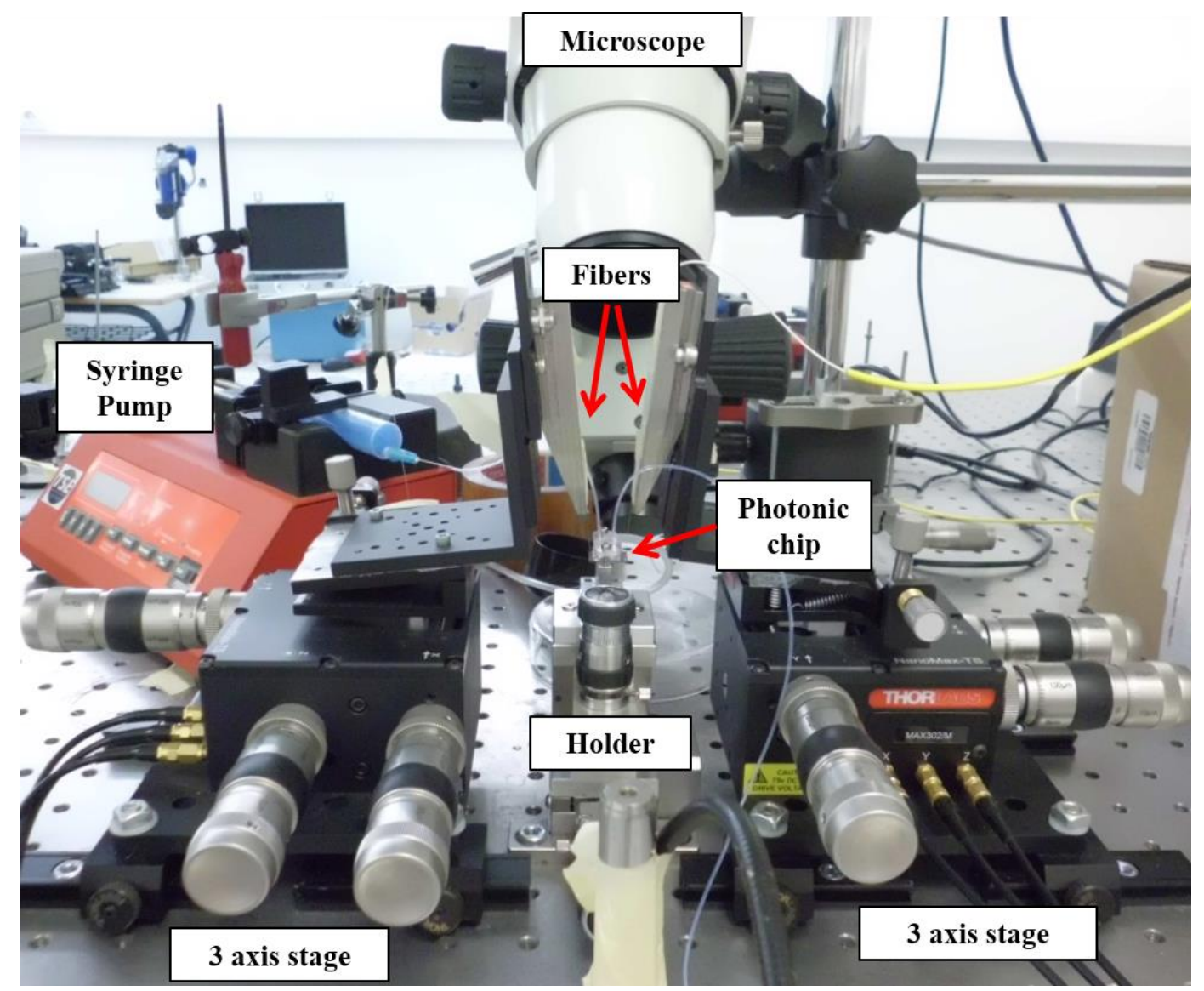

Fig. 2.17: Picture of the whole opto-fluidic measurement set-up with light coupling through grating couplers

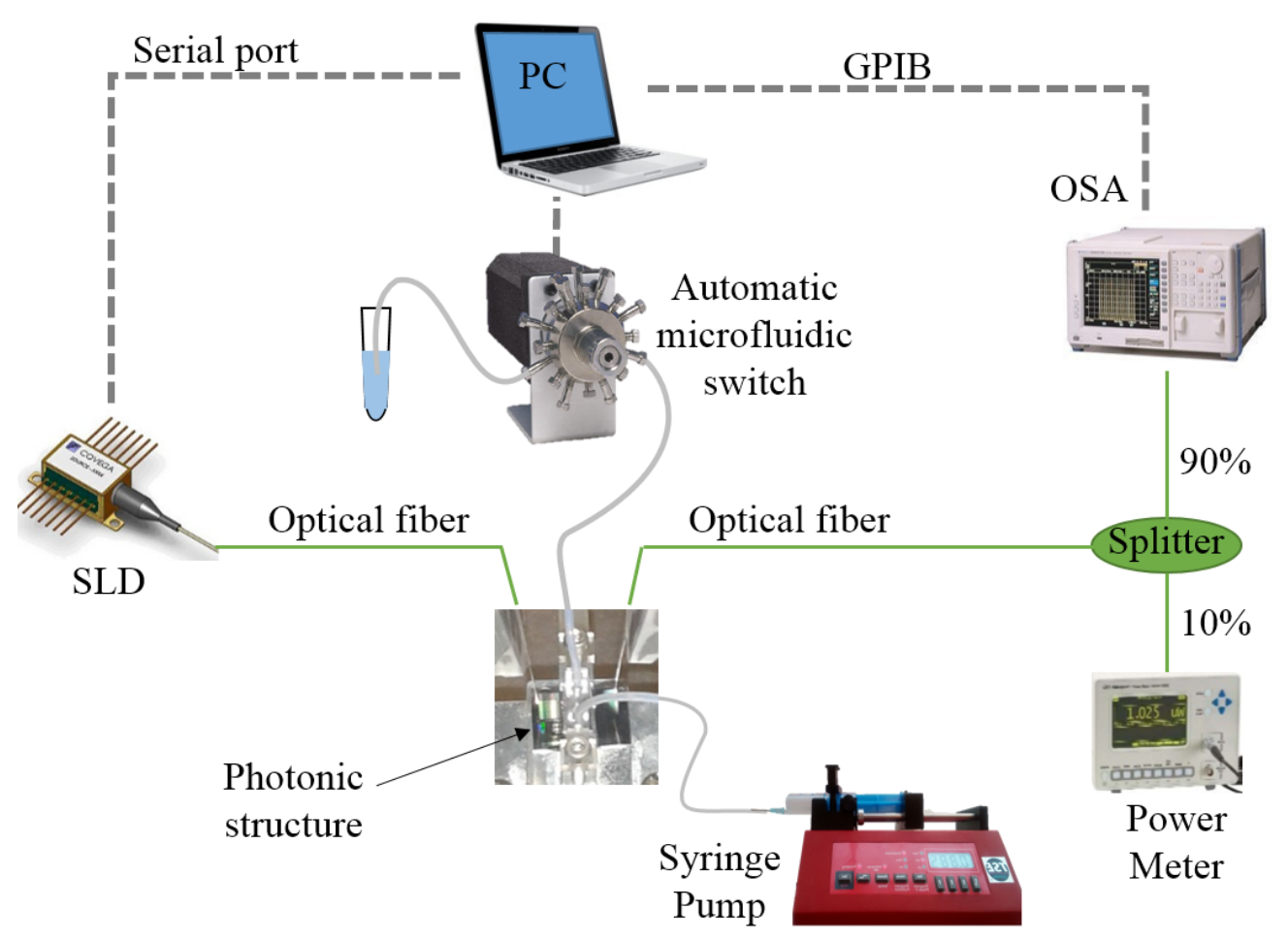

Fig. 2.18: Schematic representation of the whole experimental setup, including the fluidic and the optical sections. 


\subsection{Experimental sensing results}

As previously mentioned, the sensing experiments were based on tracking the RR spectrum, in particular of the RR resonance peaks. In Fig. 2.19 the RR spectrum is shown.

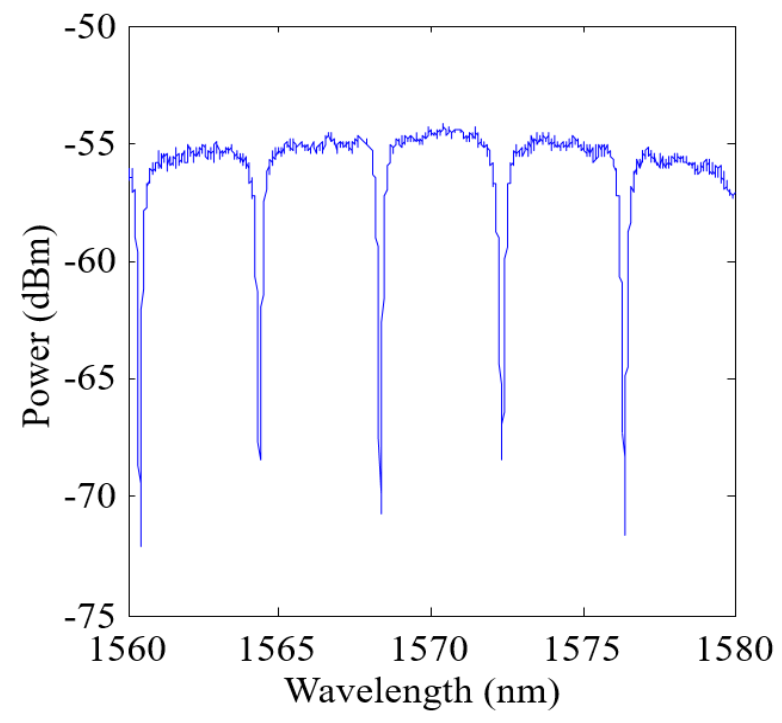

Fig. 2.19: Transmission spectrum of the through port of the RR sensor.

The development of a complete sensing platform requires numerous experiments and tests in order to obtain acceptable and reliable results. When the experimental results are theoretically acceptable and satisfactory, a careful data analysis is necessary in order to conjecture valid hypothesis upon how to develop new strategies. However, it is crucial to repeat the experiment several times in order confirm the same results and thus make appropriate conclusions. Only then, it will be possible to validate the assay and to state that the detection of the analyte is successful and repeatable. Therefore, several tests were performed by using the same photonic structure, in order to achieve acceptable results. During these measurements, several variables were changed including the surface functionalization, the measurement protocol, or the reagents to be flowed, aiming at improving the execution of the experiment and to obtain the detection of BSA with certainty.

In the first test, a bare ring resonator was bio-functionalized by directly flowing antiBSA on the surface without the use of the intermediate layer of protein A; these results will be used for comparison purposes in order to determine the performance enhancement produced by the use of the protein A intermediate layer. The second test made use of the protein A intermediate layer to immobilize the antiBSA probes over the photonic sensor. In the third test, the whole experimental procedure described in section 2.7.2 was carried out twice consecutively. In the fourth test, the specificity test was performed by flowing OVA instead of BSA. Finally, in the last test, the limit of detection for BSA recognition was determined. 
As explained in section 2.7.2, we flowed buffer after each step, so that, after the dissociation process, it was possible to study the actual layer creation. For this reason, for each step, we considered, as real wavelength shift, the residual shift after the dissociation process.

\subsubsection{BSA sensing without protein A}

AntiBSA was flowed directly over the bare ring resonator surface. Figure 2.20 shows the wavelength shift time evolution relative to (a) antiBSA and (b) BSA at a concentration of 100 $\mathrm{ng} / \mathrm{ml}$. AntiBSA was flowed for 70 minutes and BSA for 20 minutes. The antibody layer deposited on the surface caused a wavelength shift of around $100 \mathrm{~nm}$ and no BSA detection was observed. More in detail, during the evolution relative to antiBSA, a not constant rise can be observed, which means a difficulty for the adsorption of the antiBSA until a plateau was reached. When the buffer started to flow again, antiBSA desorption can be observed. When BSA was flowing, the drift was negative, what means that antiBSA was still desorbing, until reaching a plateau.
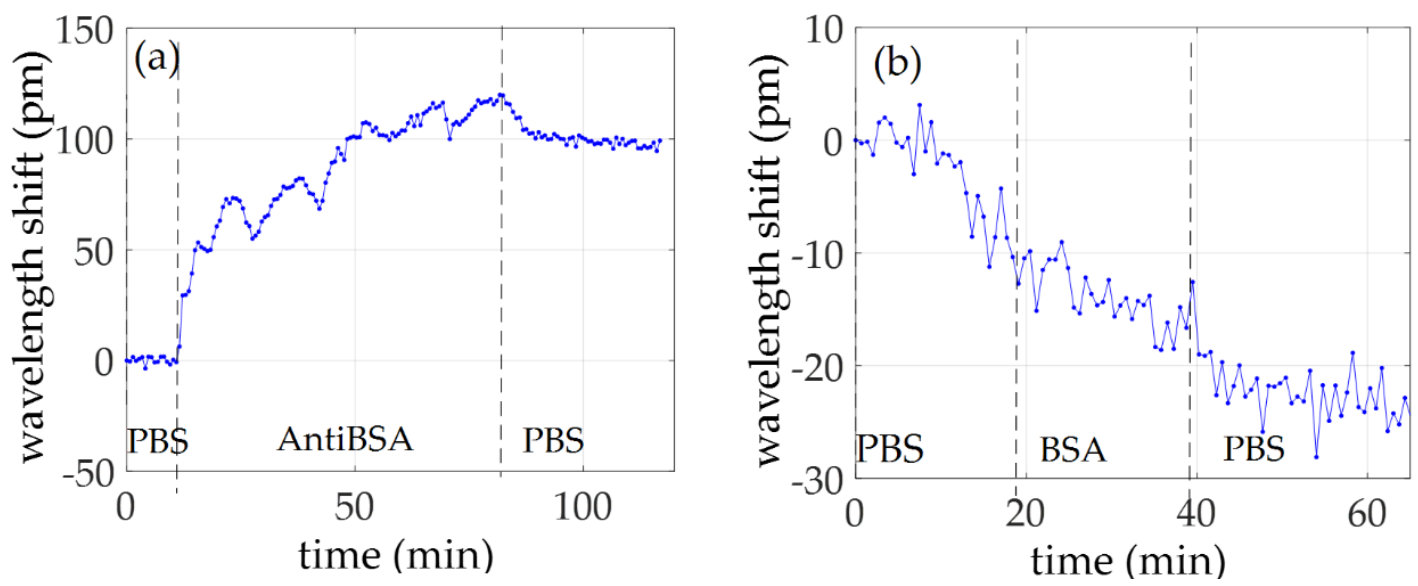

Fig. 2.20: Wavelength shift time evolution of the experiment carried out without biofunctionalizing with protein A the ring resonator surface: (a) antiBSA adsorption and (b) BSA $100 \mathrm{ng} / \mathrm{ml}$ flow.

\subsubsection{BSA sensing with protein A}

The second test was carried out by following the experimental procedure described in section 2.7.2 until the BSA detection. The aim of this experiment is to test the protein A layer creation. In Fig. 2.21, the wavelength shift time evolution is shown, where the creation of each layer (i.e., protein A, gelatin blocking, antiBSA and BSA) can be clearly appreciated. In this measurement, after the immobilization of the protein A, a wavelength shift of $110 \mathrm{pm}$ was observed, whereas in the gelatin blocking step the shift was around $270 \mathrm{pm}$. This means that not the whole sensor surface was covered by the protein A and the intermediate layer did not form correctly. After flowing gelatin (Gel in Fig. 2.21), during the PBS, a strong and constant negative drift can be appreciated. The drift represents the gelatin desorption. Next, a shift of about $220 \mathrm{pm}$ 
was measured after the antiBSA immobilization. At the end, we flowed the target BSA with a concentration of $100 \mathrm{ng} / \mathrm{ml}$ in order to carry out the detection. The wavelength shift due to BSA was around $40 \mathrm{pm}$.

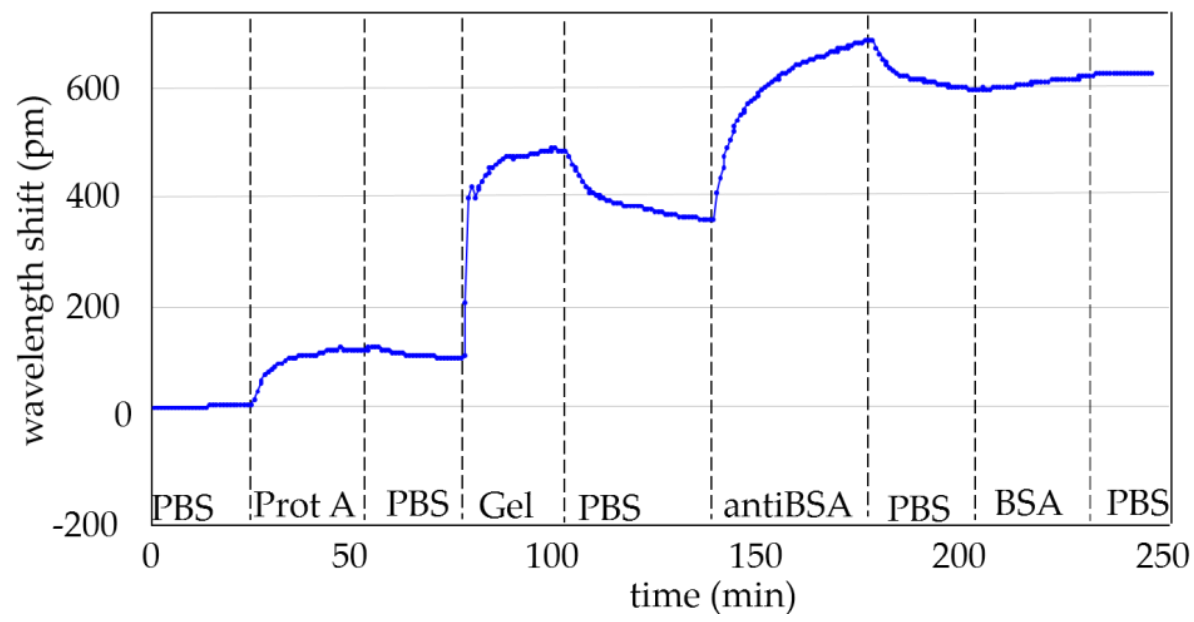

Fig. 2.21: Wavelength shift time evolution of the whole experiment carried out by following the experimental procedure until BSA detection.

It is worth noting again the poor coverage of the protein A layer over the sensor, what is clearly indicated by the significantly high shift measured for the gelatin blocking step. The reason for this poor coverage is the repulsion between the protein A, which is negatively charged for these $\mathrm{pH}$ conditions, and the thin layer of native silicon oxide present at the sensor surface, which is also negatively charged. As a consequence, a reduction of the surface adsorption degree of the protein A over the surface is produced.

\subsubsection{BSA sensing with protein A under several regeneration cycles}

Using the regeneration strategy described in section 2.7.2, several experiments were consecutively carried out monitoring in real-time the evolution of the ring resonator response. In this way, once the intermediate protein A layer was created, it was possible to perform all the tests by using the same photonic structure covered by the same protein A layer. As a matter of fact, the glycine breaks the binding between protein A and antiBSA and the protein A layer is supposed to be completely preserved.

The first experiment was carried out by following the whole experimental procedure described in section 2.7.2. However, DIW was used now as buffer for the protein A instead of PBS, in order to avoid the adsorption limitation previously described and to enhance the coverage of the sensor surface. By using DIW as buffer, whose $\mathrm{pH}$ is 5-5.5, protein A is electrically neutral and its adsorption on the sensor surface can occur without charges-related limitations. Then, before running the gelatin blocking step, buffer is changed from DIW to PBS. In Fig. 2.22 (a), the 
wavelength shift evolution of the whole experiment is shown. As shown in Fig. 2.22 (b), the immobilization of the protein A produced a wavelength shift of about $340 \mathrm{pm}$. Then, the gelatin blocking step caused a shift of only $5 \mathrm{pm}$, thus indicating that the protein A layer was properly created in the previous step. Next, a shift of around 1100 pm was measured for the antiBSA immobilization, 5 times higher than for the antiBSA immobilization step from the previous experiment. This result was due to the presence of close packed protein A layer and confirms its effectiveness. At the end, the target BSA was flowed with a concentration of $10 \mathrm{ng} / \mathrm{ml}$. A wavelength shift of around $50 \mathrm{pm}$ was measured due to the recognition of the BSA, as shown in Fig. 2.22 (c). In order to confirm such detection, the sandwich format was employed by flowing a second cycle of antiBSA. A wavelength shift around $950 \mathrm{pm}$ was then measured, almost the same value that for the first antiBSA flow, what indicated that almost all the BSA bound to the first antiBSA layer (capture antibody) was recognized by the second antiBSA flow (detection antibody).
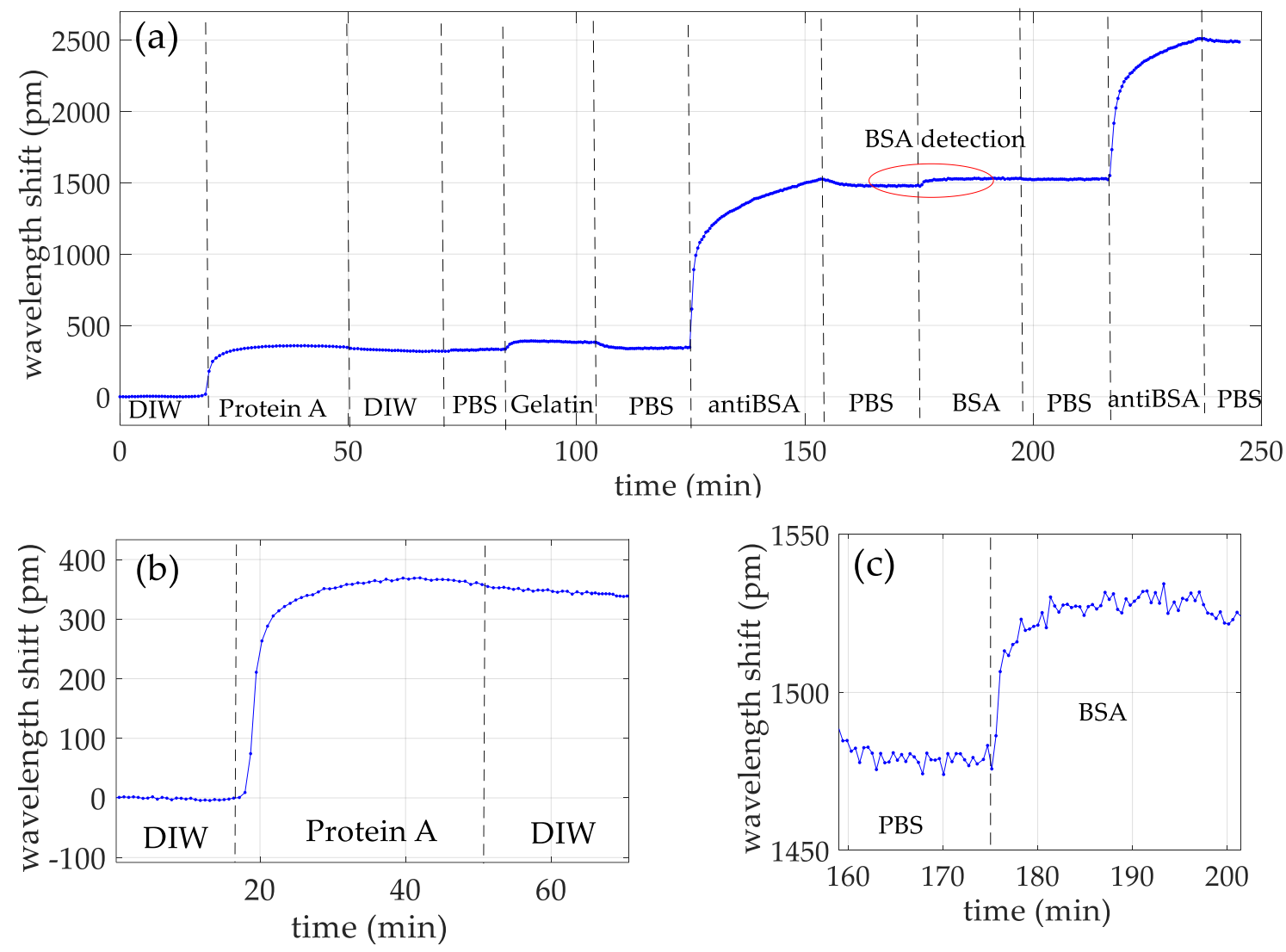

Fig. 2.22: Wavelength shift time evolution of (a) the whole experiment carried out by following our experimental procedure, (b) protein A adsorption and (c) BSA detection.

After the glycine regeneration process, a second biosensing experiment was carried out starting directly with the immobilization of the recognition antiBSA antibody. In Fig. 2.23 (a), the wavelength shift evolution is shown. A wavelength shift due to the creation of the antiBSA 
layer of $1120 \mathrm{pm}$ was observed. Then, BSA $10 \mathrm{ng} / \mathrm{ml}$ detection produced a shift of about $45 \mathrm{pm}$, as shown in Fig. 2.23 (b). Finally, the sandwich format caused a wavelength shift of around 800 pm. These results are almost the same of those obtained in the previous experiment.
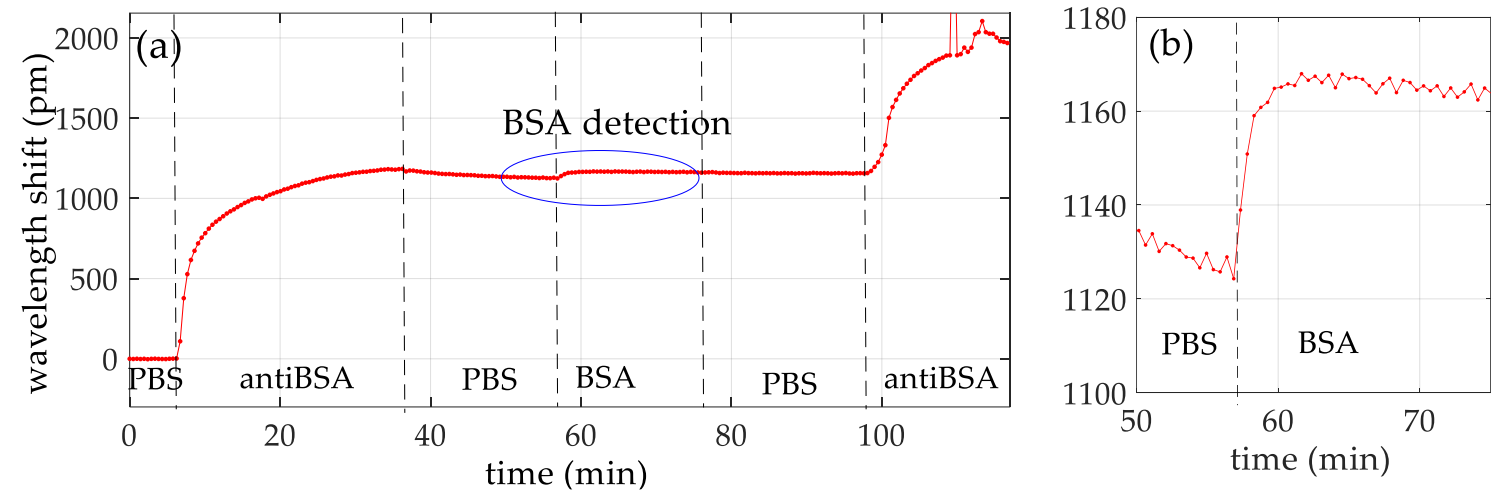

Fig. 2.23: Wavelength shift time evolution of (a) the whole second experiment and (b) BSA detection.

\subsubsection{Specificity test}

In order to demonstrate the specificity of this approach, we repeated the experiment once more, but OVA $100 \mathrm{ng} / \mathrm{ml}$ was flowed as target analyte instead of BSA. Fig. 2.24 shows the wavelength shift evolution of this specificity test. AntiBSA immobilization caused a shift around $1070 \mathrm{pm}$, whereas no shift was observed when OVA was flowed.

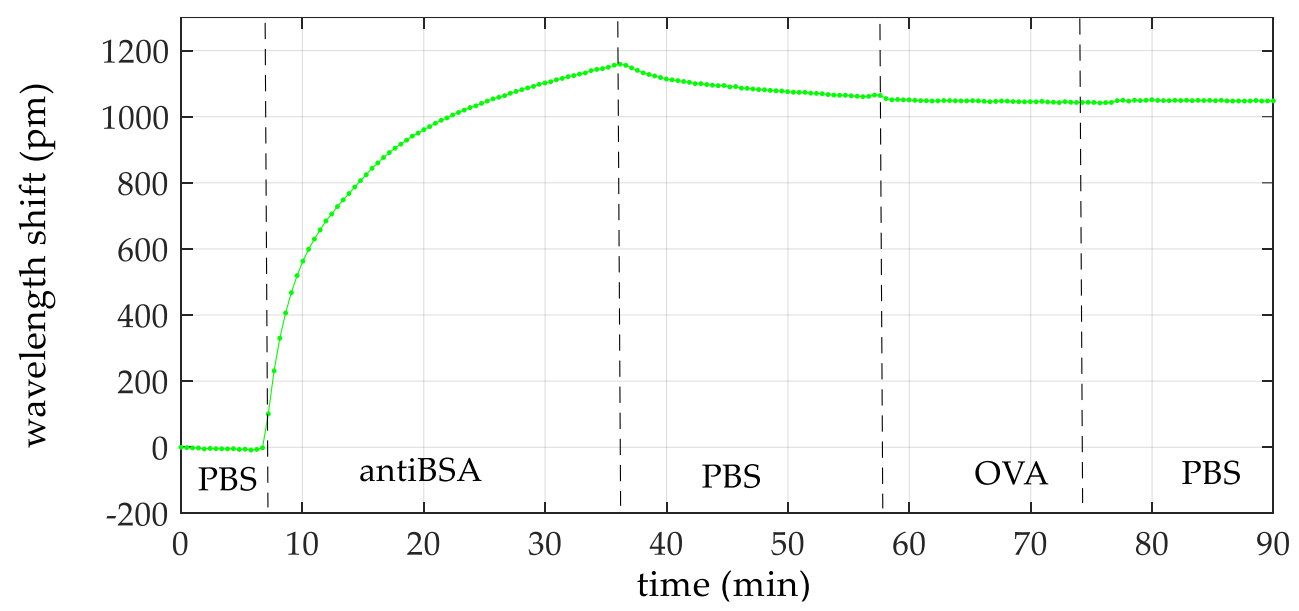

Fig. 2.24: Wavelength shift time evolution of the OVA specifity test.

\subsubsection{Experimental limit of detection of BSA}

Once the BSA detection was confirmed and the specificity of this approach was demonstrated with the previous experiments, additional experiments were carried in order to determine the limit of detection of our sensor. The lowest BSA concentration that we were able 
to detect was $1 \mathrm{ng} / \mathrm{ml}$, which correspond to molar concentration of $15 \mathrm{pM}$. This concentration produced a wavelength shift of around 7 pm, as shown in Fig. 2.25.
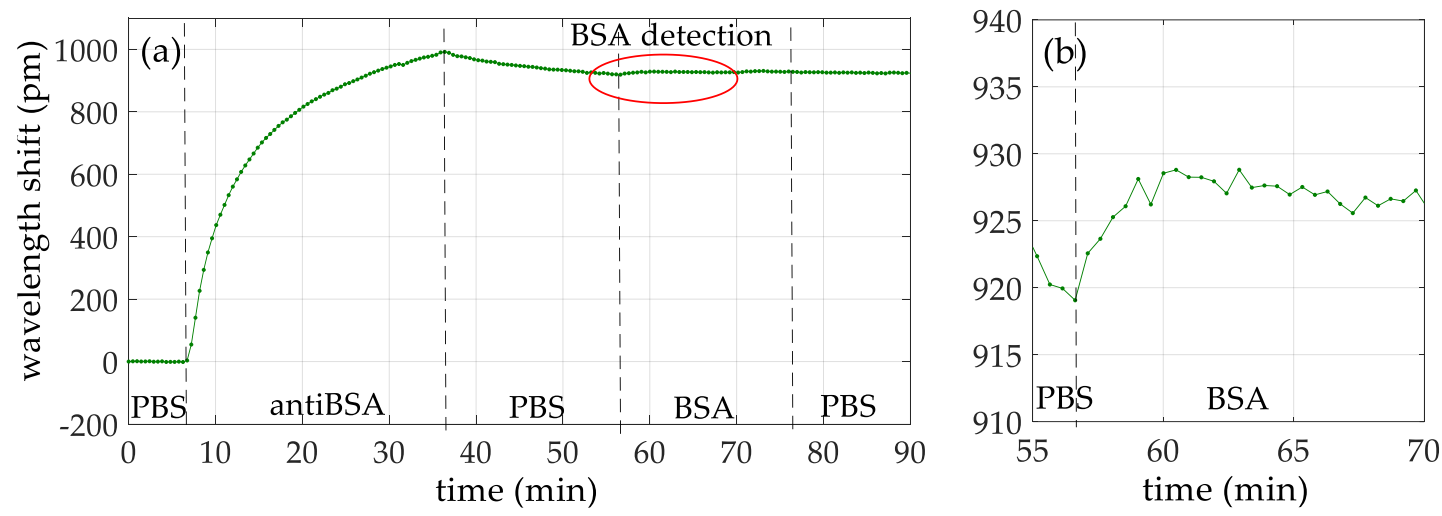

Fig. 2.25: Wavelength shift time evolution of (a) the whole fifth experiment and (b) BSA detection.

\subsection{Experimental results analysis}

\subsubsection{Effect of the protein $A$ intermediate layer}

The experimental results confirm that the oriented immobilization of antiBSA took place by covering the ring resonator surface with the protein A intermediate layer. More in detail, a wavelength shift of around 340 pm was appreciated when flowing the protein A in DIW, more than 3 times higher than for the case when the protein A was flowed in PBS, what allows having a consistent layer of protein A. Furthermore, such protein A layer was closely packed, since a shift of only 5 pm was observed when gelatin was flowed. The presence of a well-coated protein A layer allowed an optimal immobilization of antiBSA, as a wavelength shift even higher than $1100 \mathrm{pm}$ was observed and almost no antiBSA desorption was observed. Such shift was around 5 times higher than for the case when the antiBSA was immobilized on a non-optimal protein A layer, thus confirming the importance of having a good protein A intermediate layer. Furthermore, this antiBSA shift was observed in all the four experiments performed using the same protein A layer. This result indicates that the protein A layer guarantees repeatability and its effectiveness does not decrease over time. In Table 2.2, the wavelength shifts obtained for the flow of the different reagents for the three protocols used are summarized, which are also graphically represented in Fig. 2.26. It is possible to observe the large improvement obtained by flowing protein A in DIW. 


\begin{tabular}{ccccc}
\hline \multirow{2}{*}{ Protocol } & \multicolumn{3}{c}{ Wavelength shift (pm) due to: } \\
& & Protein A & Gelatin & antiBSA \\
\cline { 1 - 3 } \cline { 3 - 5 } No protein A layer & & Not flowed & Not flowed & 100 \\
Protein A in PBS & & 110 & 270 & 220 \\
Protein A in DIW & & 340 & 5 & 1100 \\
\hline
\end{tabular}

Table 2.2. Wavelength shift for the different functionalization steps for the three biofunctionalization protocols.

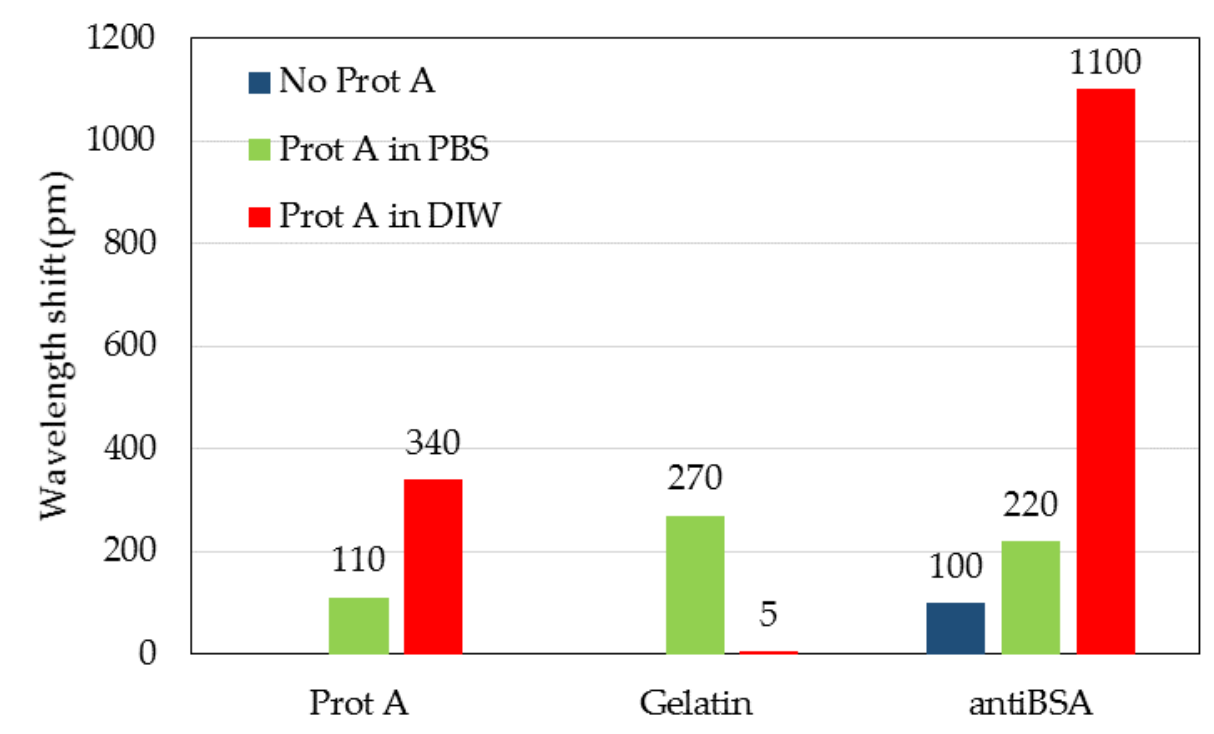

Fig. 2.26: Wavelength shift for the different functionalization steps for the three immobilization protocols used in this work.

\subsubsection{Sensitivity for antiBSA and antiBSA detection limit}

In the experiments carried out using the same protein A layer, the wavelength shift caused by the antiBSA immobilization was determined and, from that value, the sensitivity of the sensor and its minimum detectable total mass have been calculated. We have used the results obtained for the antiBSA detection because they ensure that full coverage of the sensor surface has been obtained.

The surface density of a closely-packed monolayer of antiBSA $\rho_{\text {antiBSA }}$ is given by:

$$
\rho_{\text {antiBSA }}=\frac{\mathrm{MM}_{\text {antiBSA }} / \mathrm{N}_{\mathrm{A}}}{\left(\mathrm{d}_{\mathrm{antiBSA}}\right)^{2}}
$$

where $\mathrm{MM}_{\text {antiBSA }}$ is the molecular mass of antiBSA, $\mathrm{N}_{\mathrm{A}}$ is the Avogadro's number and $\mathrm{d}_{\text {antiBSA }}$ is the average length of a molecule of antiBSA. So, taking into account that: 


$$
\left\{\begin{aligned}
\mathrm{MM}_{\text {antiBSA }} & =160 \mathrm{kDa} \\
\mathrm{N}_{\mathrm{A}} & =6.02 \cdot 10^{23} \\
\mathrm{~d}_{\text {antiBSA }} & =15 \mathrm{~nm}
\end{aligned}\right.
$$

the antiBSA monolayer density will be $\rho_{\text {antiBSA }}=1.18 \mathrm{ng} / \mathrm{mm}^{2}$.

The sensitivity for antiBSA $S_{\text {antiBSA }}$ is given by:

$$
\mathrm{S}_{\text {antiBSA }}=\frac{\Delta \lambda_{\text {antiBSA }}}{\rho_{\text {antiBSA }}},
$$

where $\Delta \lambda_{\text {antiBSA }}$ is the wavelength shift at antiBSA saturation. Since $\Delta \lambda_{\text {antiBSA }}=1120 \mathrm{pm}$, the sensitivity for antiBSA was $S_{\text {antiBSA }}=930 \mathrm{pm} / \mathrm{ng} / \mathrm{mm}^{2}$.

The detection limit of the antiBSA surface mass density $\mathrm{DL}_{\rho \text {-antiBSA }}$ is given by:

$$
\mathrm{DL}_{\rho-\text { antiBSA }}=\frac{\sigma}{\mathrm{S}_{\text {antiBSA }}},
$$

where $\sigma$ is the noise observed in the experiments. From the sensing curves obtained in the experiments, we have determined a noise value of $\sigma_{\mathrm{PBS}}=1 \mathrm{pm}$ (during the flow of PBS before the antiBSA immobilization), leading to an antiBSA surface mass density detection limit of $\mathrm{DL}_{\rho}$ antiBSA $=1.07 \mathrm{pg} / \mathrm{mm}^{2}$.

Furthermore, considering the surface area of the ring resonator $A_{R R}=\pi \cdot\left(R^{2}-r^{2}\right)=57.15$ $\mu \mathrm{m}^{2}$, also the detection limit of the antiBSA total mass $\mathrm{DL}_{\text {antiBSA }}$ was calculated as:

$$
\mathrm{DL}_{\text {antiBSA }}=\mathrm{DL}_{\rho-\text { antiBSA }} \times \mathrm{A}_{\mathrm{RR}}=60 \text { ag. }
$$

The calculated values are reported in Table 2.3.

\begin{tabular}{ccccc}
\hline $\begin{array}{c}\Delta \lambda_{\text {antiBSA }} \\
(\mathbf{p m})\end{array}$ & $\begin{array}{c}\mathbf{S}_{\text {antiBSA }} \\
\left(\mathbf{p m} / \mathbf{n g} / \mathbf{m m}^{2}\right)\end{array}$ & $\begin{array}{c}\boldsymbol{\sigma}_{\text {PBS }} \\
(\mathbf{p m})\end{array}$ & $\begin{array}{c}\mathbf{D L}_{\text {p-antiBSA }} \\
\left(\mathbf{p m} / \mathbf{m m}^{2}\right)\end{array}$ & $\begin{array}{c}\text { DL } \\
(\mathbf{a g})\end{array}$ \\
\hline 1120 & 930 & 1 & 1.07 & 60 \\
\hline
\end{tabular}

Table 2.3: Sensitivity parameters calculated from the antiBSA immobilization step.

\subsubsection{BSA detection limit and specificity}

The presence of a nonhomogeneous protein A layer led to the detection of BSA 100 $\mathrm{ng} / \mathrm{ml}$ with a wavelength shift of around $40 \mathrm{pm}$. On the contrary, the presence of a closely packed protein A layer enhanced the interaction with the target antigens to be detected, as we were able to detect very low concentrations of BSA. As a matter of fact, BSA $10 \mathrm{ng} / \mathrm{ml}$ was detected with a shift of around $45 \mathrm{pm}$, almost the same shift caused by BSA $100 \mathrm{ng} / \mathrm{ml}$ in the previous experiment. The BSA recognition was confirmed by performing a sandwich assay by flowing an additional 
cycle of antiBSA, where a wavelength shift similar to that of the first antiBSA cycle was obtained, which demonstrates that the BSA was bound to almost all the Fab regions of the antiBSA on the surface. The lowest concentration detected was $1 \mathrm{ng} / \mathrm{ml}$. The specificity of this approach was also tested by flowing OVA as target analyte.

In Table 2.4, relevant BSA detection results are reported. As it is possible to observe, the BSA concentration detected in this work is considerably lower than the ones reported in this table.

\begin{tabular}{ccccc}
\hline Sensor type & Material & Note & BSA concentration & Reference \\
\hline Ring resonator & Silicon & & $250 \mathrm{ng} / \mathrm{ml}$ & {$[53]$} \\
Ring resonator & Silicon nitride & Slot & $400 \mathrm{ng} / \mathrm{ml}$ & {$[60]$} \\
Ring resonator & Polymeric & Through Flow & $10 \mathrm{nM}$ & {$[61]$} \\
Mach-Zehnder & Optical fiber & & $250 \mathrm{ng} / \mathrm{ml}$ & {$[62]$} \\
\hline
\end{tabular}

Table 2.4. Review of experimental results reported in BSA sensing.

Figure 2.27 summarizes the sensing results obtained for the four experiments carried out using the same protein A layer. For a better comparison, a wavelength shift of 0 is considered for the moment when the target analytes begin to flow over the photonic sensor. As it can be clearly observed in Fig. 2.27 (b), a shift is produced when BSA is flowed for all the concentrations, while no variation in the trend of the sensing signal is observed when OVA is flowed in the experiment. Figure 2.28 depicts the measured wavelength shift as a function of the target BSA concentration.
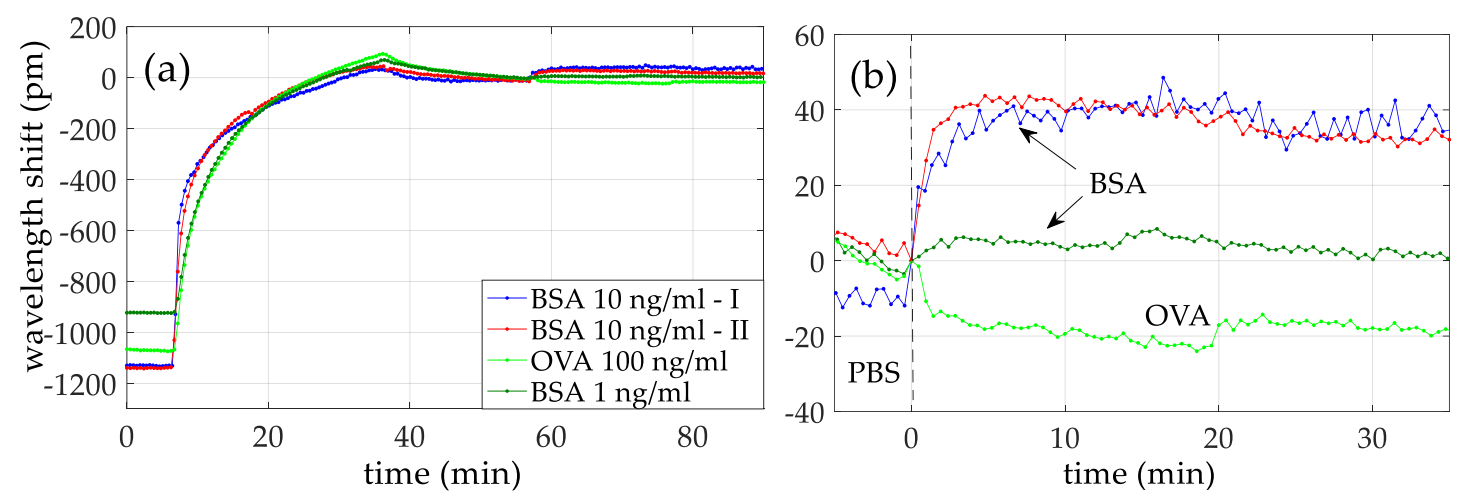

Fig. 2.27: Comparison of the wavelength shift evolution of all the experiments: (a) antiBSA immobilization and (b) target analyte detection. 


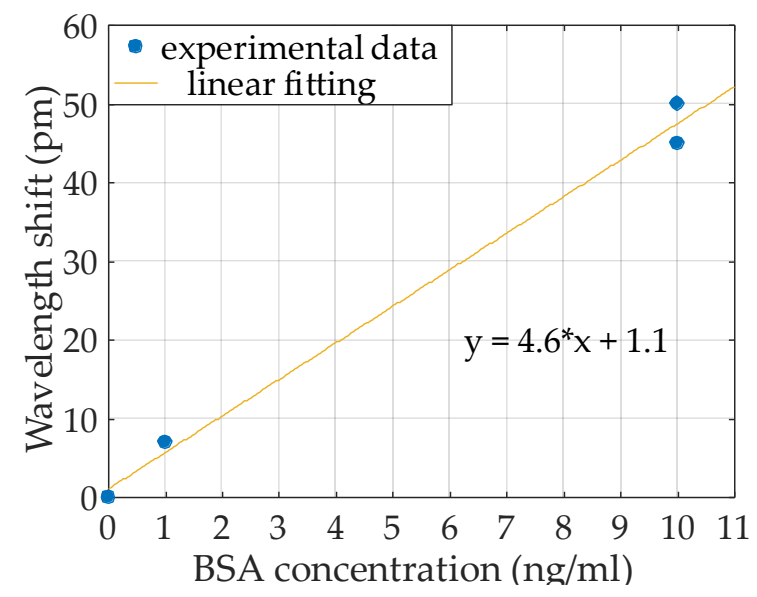

Fig. 2.28. Wavelength shift values as a function of the BSA concentration

\subsection{Conclusions}

This chapter has been focused on the study of an experimental antibody immobilization approach for photonic sensors based on the use of an intermediate layer of protein A to improve that immobilization and on the use of a RR as sensing element.

We have begun introducing the fundamentals of the RR and its main parameters. The sensing technique adopted using this sensor was based on the tracking the RR resonance peaks. After that, we have described our RR and the chemical reagents employed in this work. The procedure adopted to flow all the reagents over the sensor surface consisted in creating a protein A layer to allow the proper antibody orientation, blocking the uncovered sensor surface with gelatin, immobilizing antiBSA and detecting BSA. Then, we have presented the automated experimental set-up that was used for the experimental characterization of the RR-based sensing structures and to carry out the experiments. The setup was composed by a fluidic and an optical part working simultaneously, in order to flow the reagents on the sensor and, at the same time, monitoring the RR spectrum evolution.

Finally, we have reported the experimental results. We tested the use of the protein A layer and confirmed its usefulness to obtain a well-coated surface of antibodies oriented towards the target sample to be analysed, thus enabling the development of high sensitivity biosensors. The experimental validation was carried out by means of BSA detection experiments. By covering the sensor surface with a closely packed intermediate protein A layer, antiBSA immobilization was considerably improved. Such immobilization improvement led to the detection of lower BSA concentrations, such as $1 \mathrm{ng} / \mathrm{ml}$. Therefore, these results confirm the good performance in terms of sensitivity that can be obtained using a very simple, cheap and easy-to-implement biofunctionalization approach. Furthermore, we proved the repeatability and the durability over time of this approach, since several experiments were carried out using the same protein A layer and 
the same results were obtained. We also confirmed the specificity of the experimental procedure, where we used gelatin as blocking agent in order to avoid unspecific adsorption. Moreover, the creation of each layer was monitored in real-time thanks to the opto-fluidic setup developed to carry out all the experiments that we have reported.

To conclude, it is important to point out that this immobilization approach was very simple and fast, since the protein A layer was created over the ring resonator surface simply by physical immobilization. In fact, we were able to create such layer in a few minutes just by flowing protein A in DIW over the ring resonator surface, allowing, furthermore, the in real-time monitoring of the layer creation. 


\section{Chapter 3}

\section{Sensing using porous silicon $1 D$ periodic structure}

\subsection{Introduction}

In chapter 1, we have seen that traditional photonic sensors based on the evanescent wave mechanism are limited by the fact that just a little amount of the field propagating inside the structure is exploited for sensing purposes. We have also seen that the presence of the bioreceptor layer separates the target analytes from the sensor surface making the analyteevanescence wave interaction weaker. However, as time goes by, the use of higher sensitive sensing devices in sensing applications is required. For this reason, it is necessary to constantly look for a solution to the present limitations. In this context, we find a solution in the use of PS for the development of more sensitive optical sensors. In fact, as mentioned in chapter 1, in PS structures, the light-matter interaction occurs directly inside the structure itself what allows to exploit all the field propagating in the structure.

In this chapter, we present the development of a highly sensitive porous silicon microcavity (PSMC). In section 3.2, we introduce the fundamentals of PS 1D periodic structures. 
Afterwards, in section 3.3, an overview of several works of sensing using PS structures is reported. After the theoretical introduction, we move to the experimental part of the chapter. In section 3.4 all the experimental fabrication process is described. The experimental opto-fluidic setup developed to carry out the experiments in real-time is presented in section 3.5. The experimental results have been organized in two parts. First, in section 3.6, we reported the sensing results obtained using as optical sensors Bragg reflectors, and, then, in section 3.7, using a PSMC. Finally, in section 3.8, we present our conclusions.

\subsection{Fundamentals of PS 1D periodic structures}

Light propagation can be manipulated by changing the refractive index of the medium along the propagation direction. In fact, when the medium where the electromagnetic wave propagates is periodic along the direction of propagation instead of homogeneous, the wave propagation behaviour completely changes. In a properly designed periodic structure, the wave cannot propagate through the material in a range of wavelengths and it is reflected. This range is the reflection band. The width and position of the reflection band is highly dependent on the refractive index configuration of the whole structure. Accordingly, by properly designing a PS multilayer, it is possible to control the reflectance band. To this aim, the PS layer physical parameters must be controlled and carefully selected. These parameters are the porosity, the pore dimeter and the thickness of the PS layer.

- Porosity: It is a crucial parameter, since it confers the RI of the porous layer. High porosities determine low RI values and vice versa. It is defined as the empty pore volume to total volume ratio.

- Pore diameter: From this parameter, it is possible to classify the PS morphology.

- Micropores have pore diameters below $2 \mathrm{~nm}$ and present a morphology like a sponge.

- Mesopores have pore diameters between 2 and $50 \mathrm{~nm}$ and present a morphology like a tree.

- Macropores have pore diameters above $50 \mathrm{~nm}$ and are straight with a smooth surface.

The diameter of the pore determines the surface to volume ratio. Lower pore diameters determine a high surface to volume ratio and vice versa. For this reason, in order to increase the usable surface to biofunctionalize it is better a low pore diameter.

- Thickness: Together with the porosity, it determines the position of the reflected band. For higher layer thicknesses, the reflected band will be located at higher wavelengths. 
A basic configuration of periodic PS multilayer commonly used in sensing applications is the Bragg reflector. Figure 3.1 shows a schematic illustration of the working principle of a Bragg reflector. A Bragg reflector is a periodic structure formed by alternating PS layers of high $\left(\mathrm{n}_{\mathrm{H}}\right)$ and low $\left(\mathrm{n}_{\mathrm{L}}\right)$ refractive index. The periodicity of the structure provides a reflectance spectrum characterized by a reflectance band with a maximum at the Bragg wavelength $\lambda_{\mathrm{B}}$. The thicknesses of the $n_{H}$ and $n_{L}$ layers, $d_{H}$ and $d_{L}$, respectively, satisfy the relation:

$$
\lambda_{B}=\frac{2\left(n_{H} \cdot d_{H}+n_{L} \cdot d_{L}\right)}{m} .
$$

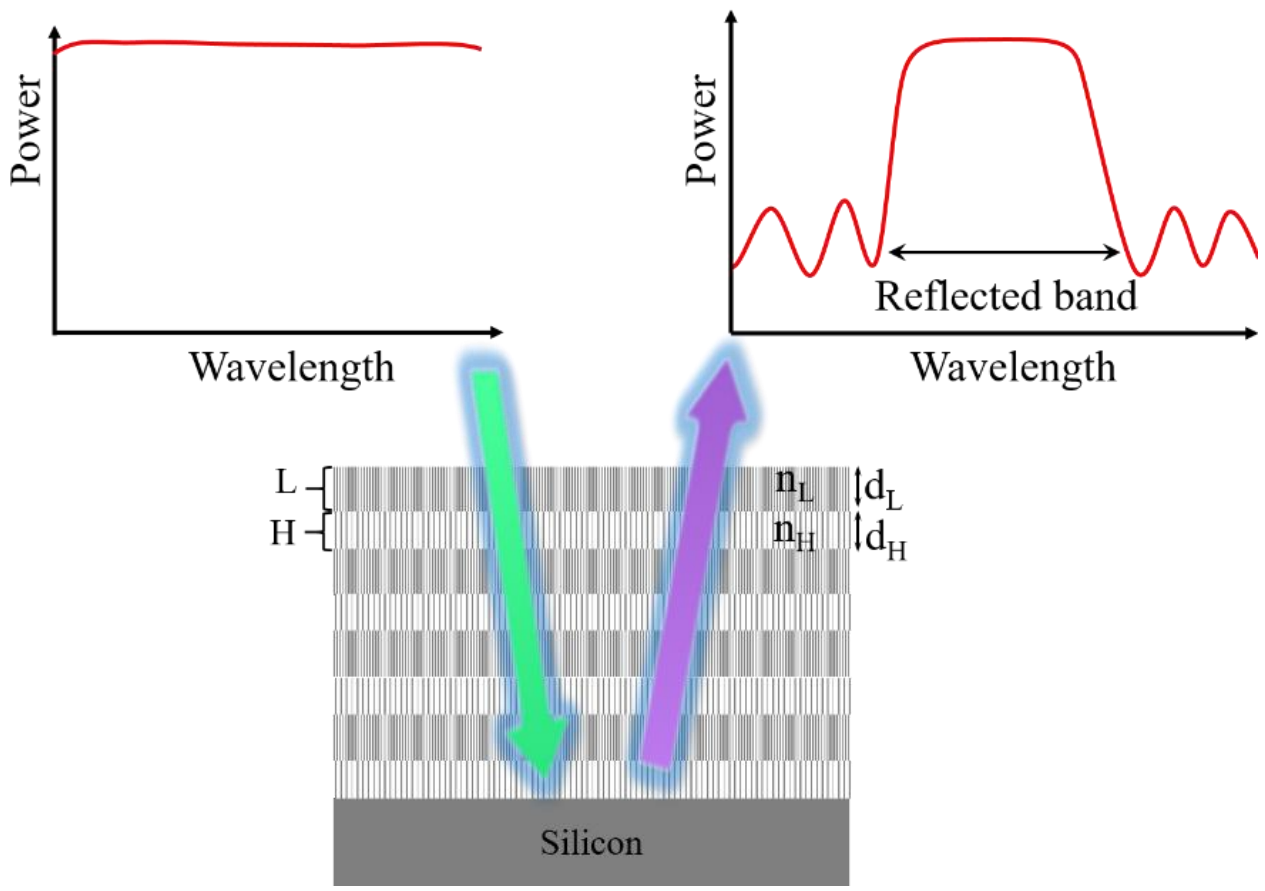

Fig. 3.1: Schematic illustration of the working principle of a Bragg reflector.

Another configuration widely employed in sensing applications is the porous silicon microcavity (PSMC). Figure 3.2 shows a schematic illustration of the working principle of the PSMC. This structure is obtained by placing a central defect layer, also called microcavity (MC), between two Bragg reflectors. The presence of the central defect gives rise to a resonance peak in the reflectance spectrum at $\lambda_{\mathrm{c}}$. The optical thickness $\mathrm{d}_{\mathrm{c}}$ is typically selected as one half the resonant wavelength of the $\mathrm{MC}$ and satisfies the relation:

$$
\mathrm{d}_{\mathrm{c}}=\lambda_{\mathrm{c}} / 2
$$




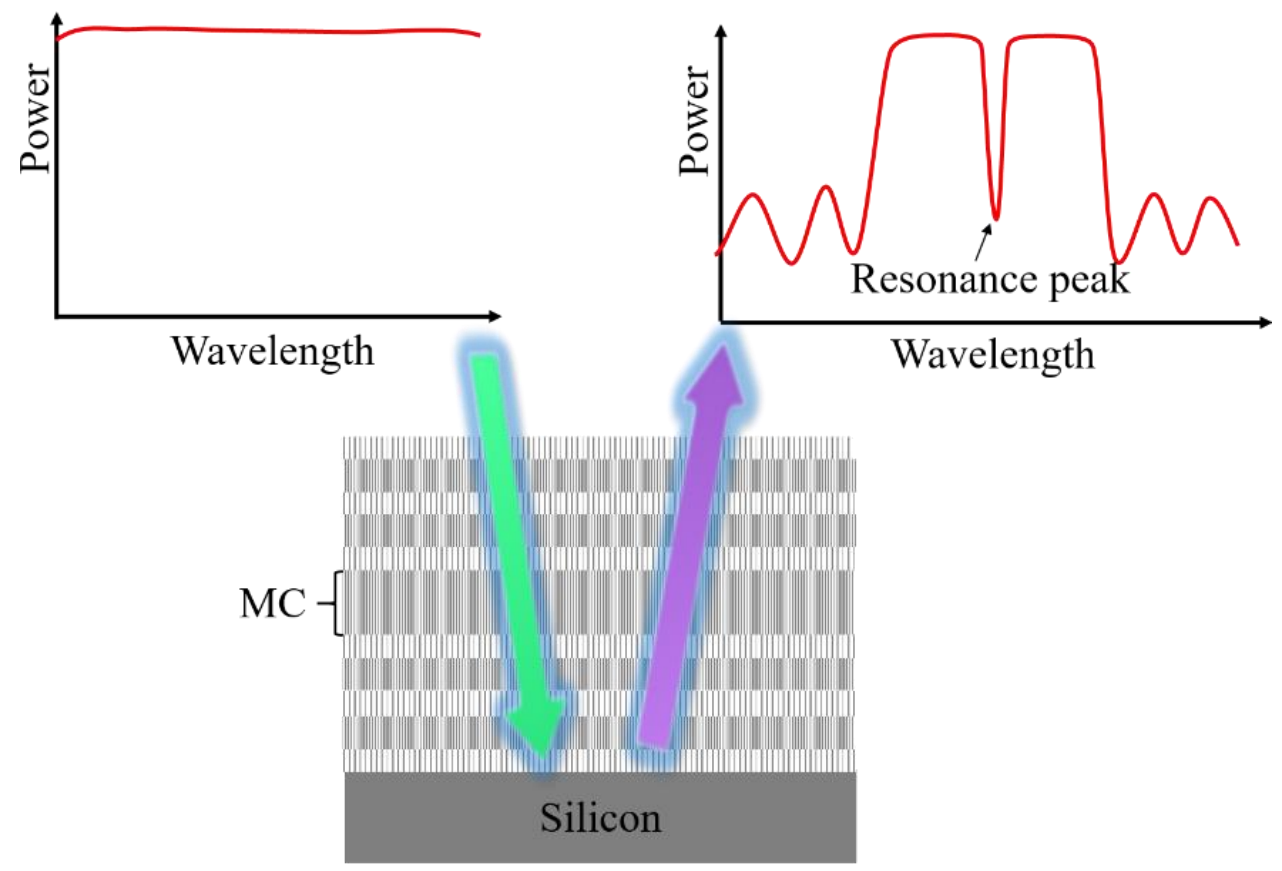

Fig. 3.2: Schematic illustration of the working principle of a porous silicon microcavity.

The sensing mechanism of a PS 1D periodic structure is schematically shown in Fig. 3.3. When the target substance fills the pores, the RI of the PS layers changes, leading to a shift of the reflectivity spectrum.

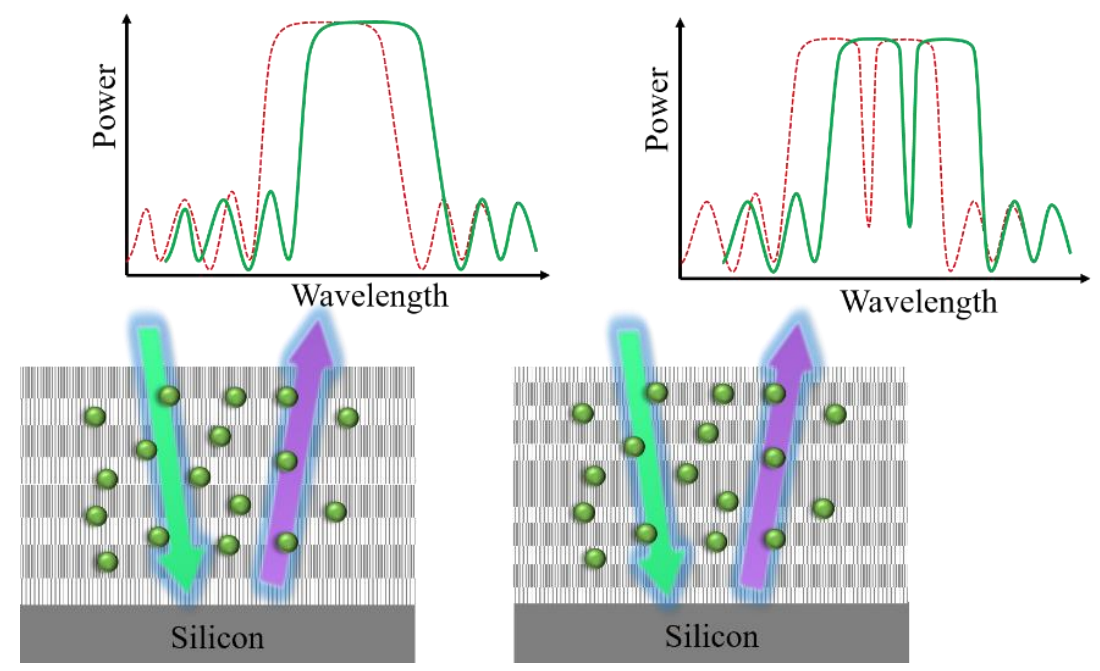

Fig. 3.3: Schematic illustration of the sensing working principle of a PS 1D periodic structure.

\subsection{PS 1D periodic structures overview}

PS has been used for sensing purposes since the first years of 1990. Applications where PS confirmed its benefits and where it has reached a great success are gas sensing and biosensing. In the last two decades, a large number of works have been presented and, in the last years, several 
reviews have reported them and highlighted their results [63-65]. For example, Pacholski, in [63] reported chronologically and described carefully the most relevant results obtained in gas sensing and biosensing using PS periodic structures.

PS sensors have found a considerable interest in gas sensing due to the ability of nanoporous materials to concentrate vapor by microcapillary condensation: vapor directly condensate to liquid on the curved walls of the pores. One of the first gas sensing demonstrations using a 1D PS reflectivity spectrum was reported by Snow at al. [66]. Large wavelength shifts were observed in the reflectivity spectrum of the Bragg reflector as a consequence of the exposure of the structure to vapor from organic solvents. Soon after, Pavesi et al. reported relevant results regarding the detection of different solvents and the discrimination of them by using a PSMC as a gas sensor and measuring the reflectivity spectrum and the photoluminescence intensity at the same time [67]. The shift of the PSMC resonance peak depended on the organic compound refractive index leading to the study of the sensitivity, while the luminescence intensity depended on the low-frequency dielectric constant leading to the discrimination of the solvents and, thus, to the specificity of the sensors.

PS established itself also in biosensing applications. The first PS biosensor was developed by Lin et al. in 1997 [68]. They detected small molecules, as biotin and digoxigenin, DNA oligomers, and proteins, as streptavidin and antibodies, at pico- and femtomolar concentrations. Starting from 2000, several research groups focused on the development of novel PS reflectivity based sensors. Some of these works were oriented to the study of the sensitivity of these sensors and others to the detection of biomolecules, optimizing the biofunctionalization of PS. High sensitivity values, higher than 300 nm/RIU, were obtained [69-72]. In 2005, DeLouise at al. investigated the sensitivity of a series of PSMCs in order to characterize the response to changes in bulk refractive index as a function of the design parameters of the PSMCs and obtained a sensitivity of $425 \mathrm{~nm} / \mathrm{RIU}$ [69]. Furthermore, they investigated also the immobilization of glutathione-S-transferase, achieving a detection limit of $50 \mathrm{pg} / \mathrm{mm}^{2}$. One of the highest sensitivities was reported by Rendina et al., with a value of $\sim 470 \pm 40 \mathrm{~nm} / \mathrm{RIU}$, using a Bragg reflector for gas sensing [70]. Several relevant results have been reported in the field of biosensing using PSMCs as sensing structures. Zhang et al. fabricated a PSMC biosensor based on SOI wafer for DNA detection and achieved a detection limit of $43.9 \mathrm{nM}$ [73]. Recently, Zhao et al. reported a remarkable work [74], where they demonstrated a flow-through sensing platform based on openended PSMC membranes and its use for streptavidin detection. The molecular binding kinetics results were compared with those obtained by using a traditional close-ended PSMC and an improvement of 6-fold of the sensor response was demonstrated. 


\subsection{PS 1D periodic structures fabrication}

\subsubsection{PS 1D periodic structures design}

In the design phase, the parameters to take into account to develop the required structure are the RI, $n_{H}$ and $n_{L}$, and the thickness, $d_{H}$ and $d_{L}$, of the layers. These parameters have to satisfy the relation $2\left(n_{H} \cdot d_{H}+n_{L} \cdot d_{L}\right)=m \cdot \lambda_{B}$, where $m$ is the order of the Bragg condition.

To determine the structure parameters $\mathrm{n}_{\mathrm{H}}, \mathrm{n}_{\mathrm{L}}, \mathrm{d}_{\mathrm{H}}$, and $\mathrm{d}_{\mathrm{L}}$, a model based on the Transfer Matrix Method (TMM) [75-76] was used. This model allows to calculate the transmission and the reflection spectra of a one-dimensional structure consisting of an arbitrary number $\mathrm{N}$ of alternated porous silicon layers with different porosities and thicknesses.

When a plane wave of wavelength $\lambda$ impinges on a stack of layers of various refractive indices $n_{i}$ and thicknesses $d_{i}$, for every boundary there will be a transmitted and a reflected component of the electric field (if the refractive indices are complex, there will be also an absorbed component). Hence, the electric field distribution will suffer a transformation depending on the refractive indices on both sides of the interface:

$$
E_{m+1}=\left(\begin{array}{l}
E_{m+1}^{+} \\
E_{m+1}^{-}
\end{array}\right)=\frac{1}{2}\left(\begin{array}{cc}
1+\frac{n_{m-1}}{n_{m}} & 1-\frac{n_{m-1}}{n_{m}} \\
1-\frac{n_{m-1}}{n_{m}} & 1+\frac{n_{m-1}}{n_{m}}
\end{array}\right)\left(\begin{array}{c}
E_{m}^{+} \\
E_{m}^{-}
\end{array}\right)
$$

where the index + refers to the direction of propagation of the incident light and - to the opposite one. Moreover, the effect of the electric field propagation across a homogenous layer can be expressed as:

$$
\left(\begin{array}{c}
E_{m}^{+} \\
E_{m}^{-}
\end{array}\right)^{\prime}=\left(\begin{array}{cc}
e^{i k_{m x} d_{m}} & 0 \\
0 & e^{-i k_{m x} d_{m}}
\end{array}\right)\left(\begin{array}{c}
E_{m}^{+} \\
E_{m}^{-}
\end{array}\right)
$$

with $\mathrm{k}_{\mathrm{mx}}$ being the $\mathrm{x}$ component of the wave vector. The contribution of every layer can be expressed with these matrices:

$$
E=\left(\begin{array}{ll}
M_{11} & M_{12} \\
M_{21} & M_{22}
\end{array}\right) E_{0}
$$

Having the electric field described this way, the reflectance is given by:

$$
\mathrm{R}=\left|\frac{\mathrm{M}_{21}}{\mathrm{M}_{11}}\right|^{2}
$$

For the calculation of porosity and refractive index of the PS layer, we based on the effective medium approximation. In this $\mathrm{PhD}$ Thesis, light sources emitting at a wavelength in 
the infrared (IR) range were used. More in detail, we worked with wavelengths included between $1450 \mathrm{~nm}$ and $1650 \mathrm{~nm}$. At this wavelength range, light perceives a porous layer presenting pores with a diameter included between $2 \mathrm{~nm}$ and $200 \mathrm{~nm}$ as an effective medium. Nowadays, several effective medium approximation methods are considered to calculate the porosity and the refractive index of PS layers, such as Maxwell-Garnett's, Looyenga's or Bruggeman's [77]. In Fig. 3.4, the RI versus porosity relation of these approaches are shown.

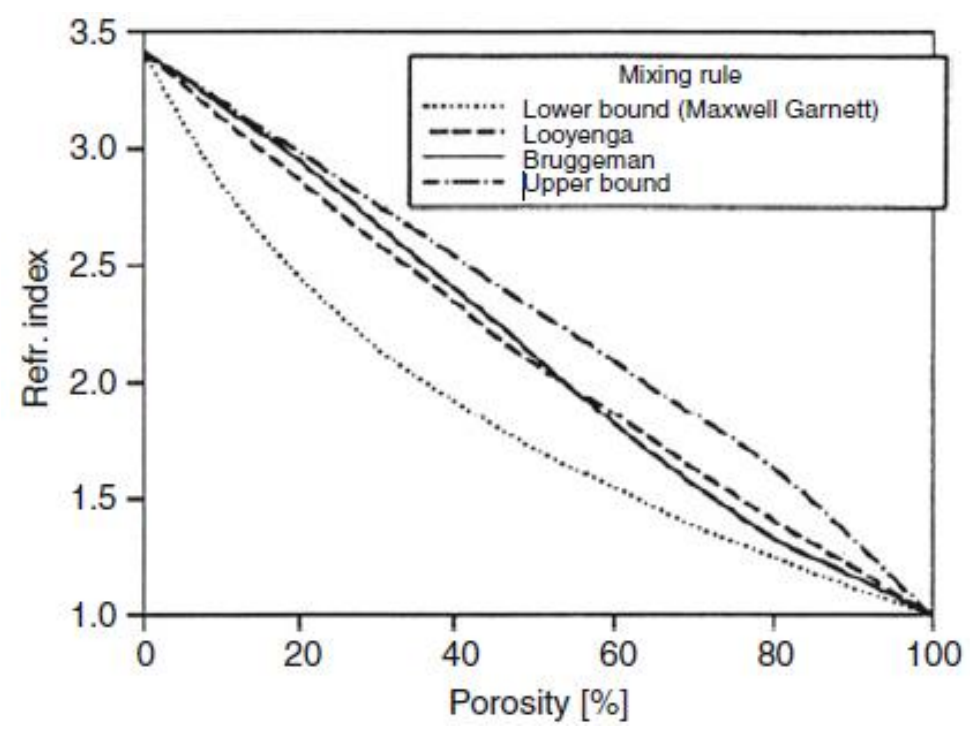

Fig. 3.4: RI versus porosity from [77].

The Bruggeman effective medium approximation considers connected networks for medium-high porosities, as those considered in this $\mathrm{PhD}$ Thesis. For this reason, the Bruggeman approximation was considered in the simulation process to calculate the porosity $\mathrm{P}$ or the effective refractive index $n_{\text {eff }}$ of the porous layer to be fabricated. The Bruggeman approximation is given by the equation 3.7 [78], where $n_{\text {fill }}$ and $n_{S i}$ are the RIs of the substance filling the pores and of Silicon, respectively:

$$
\mathrm{P} \frac{\mathrm{n}_{\text {fill }}^{2}-\mathrm{n}_{\text {eff }}^{2}}{\mathrm{n}_{\text {fill }}^{2}-2 \mathrm{n}_{\text {eff }}^{2}}+(1-\mathrm{P}) \frac{\mathrm{n}_{\mathrm{Si}}^{2}-n_{\text {eff }}^{2}}{n_{\mathrm{Si}}^{2}-2 n_{\text {eff }}^{2}}=0
$$

\subsubsection{Electrochemical system}

Nowadays, several methods are implemented to porosificate Silicon, including electrochemical etching, chemical stain etching and chemical vapor etching [79]. However, the most common method used for the formation of PS is electrochemical etching, since it has been demonstrated to guarantee reproducibility and reliability on the achievement of the thickness and porosity of the formed porous layers. In this PhD Thesis, the PS structures were fabricated by electrochemical etching. 
To perform the electrochemical etching, an electrochemical system is needed. Basically it consists of an electrolyte, a cathode and an anode electrodes and an electrochemical cell. The electrolyte is contained in the electrochemical cell, where the etching is performed. The two electrodes are placed at a distance of a few millimeters in order to allow the charge current to flow from an electrode to the other.

- Electrolyte: It enables the ionic transport from the cathode to the anode and must contain chemical species allowing anodic Si dissolution. Usually, the electrolyte is a hydrofluoric acid (HF) solution. More in detail, HF is sold in an aqueous solution with up to $50 \%$ of HF. PS can be fabricated by using only HF diluted in deionized water (DIW). However, using this type of electrolyte two issues can take place. On the one hand, PS surface is highly hydrophobic, due to the hydrogen termination of PS. This avoids water to completely fill the pores. On the other hand, during the electrochemical etching, hydrogen evolution is in progress. This causes the formation of bubbles in the pores, avoiding the water entering in the pores. In order to overcome such issues, ethanol $(\mathrm{EtOH})$ is typically added to the HF solution, reducing the surface tension of the electrolyte and thus allowing it to enter the pores as they are formed.

- Cathode: It supplies electrons to the electrolyte. It is made of a highly conductive material and resistant to HF. The most used material is platinum. In electrochemistry, this electrode is called counter electrode.

- Anode: It is the Silicon itself. In electrochemistry, this electrode is called working electrode.

- $\quad$ Electrochemical cell: It is the container where the electrolyte and the electrodes are located and where the process is performed. As the electrolyte is a HF solution, the material of the cell is usually Teflon, which is resistant to HF at high concentrations.

In this $\mathrm{PhD}$ Thesis, in order to perform the electrochemical etching of silicon and, thus, fabricate our PS structure, we built up a homemade electrochemical system. Our system was based on a vertical two electrode cell configuration, as shown in Fig. 3.5. We considered this configuration since it allows to achieve a high uniformity of the layer porosity. First of all, a $20 \times 20 \mathrm{~mm}^{2} \mathrm{Si}$ sample was used as a sample to porosificate and, at the same time, as an anode. A copper sheet was employed as a backside contact for the Si sample. A Teflon bottle (from Labbox) was used to build the electrochemical cell to contain the electrolyte. As electrolyte, we used a HF solution. A hole with a diameter of $12 \mathrm{~mm}$ was made at the base of the bottle in order to have an etching area of $1.13 \mathrm{~cm}^{2}$. To hermetically seal the cell, a Viton O-Ring was placed between the Si sample and the Teflon cell. A platinum wire with a diameter of $0.25 \mathrm{~mm}$ (from Chempur) was used as a cathode. More in detail, the platinum wire was moulded in the form of a spiral, in order 
to have an electrochemical etching as homogenous as possible. The spiral diameter was around $16 \mathrm{~mm}$ in order to provide an area (around $2 \mathrm{~cm}^{2}$ ) wider than the etching area and minimize the boundary effects during the PS layer formation process.

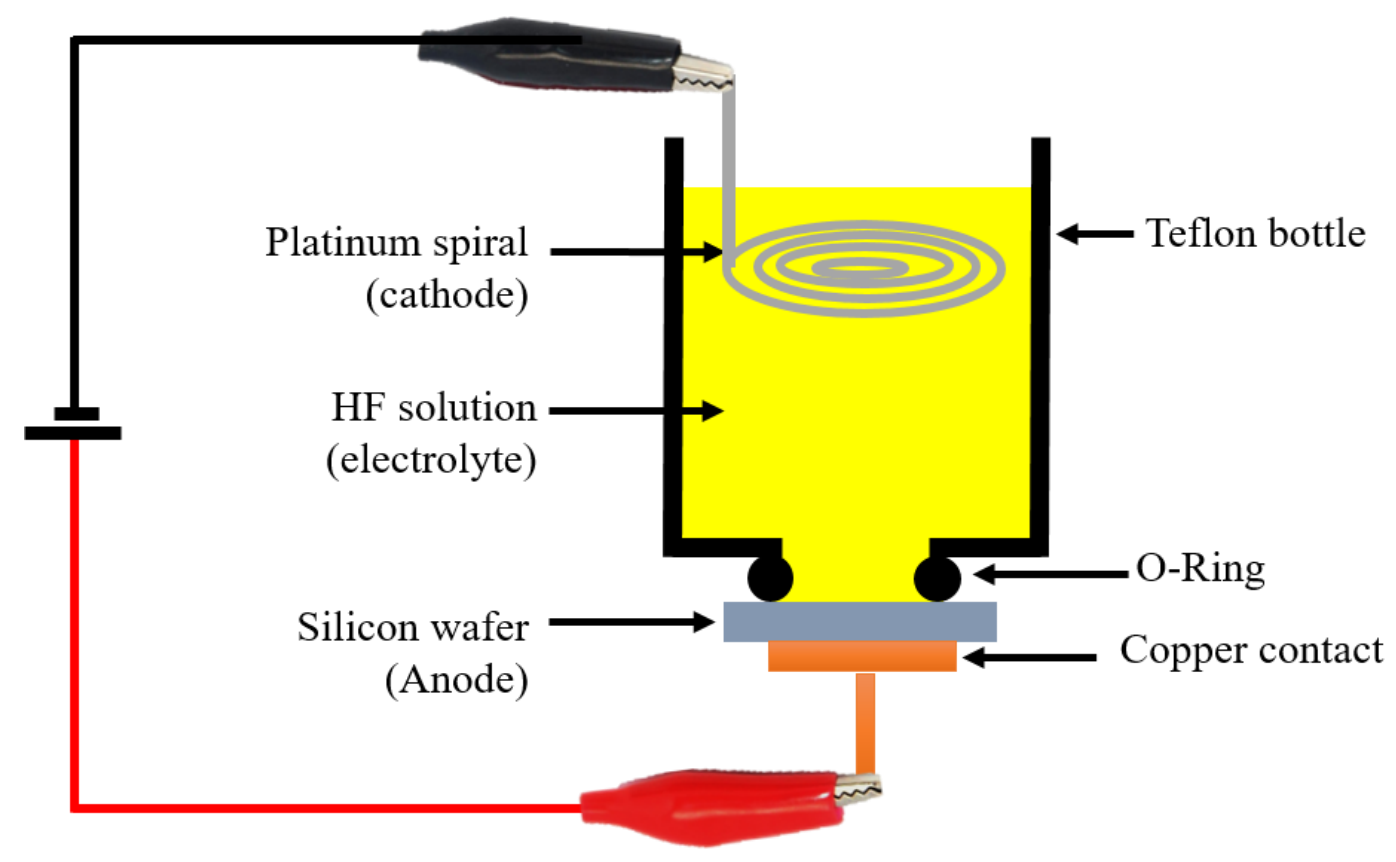

Fig. 3.5: Schematic representation of the vertical two electrodes electrochemical system configuration.

In Fig. 3.6, the main components of the electrochemical system are shown and its building up is illustrated in Fig. 3.7. The final configuration is shown in Fig. 3.8.

(a)

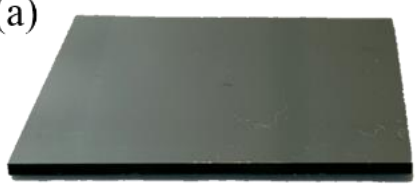

(c)

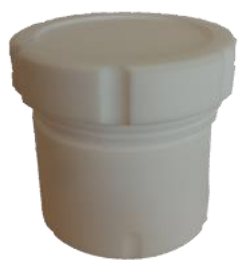

(b)

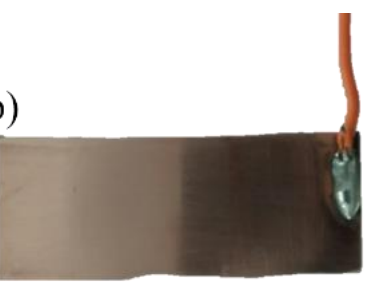

(d)

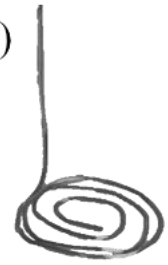

Fig. 3.6: Electrochemical components used to carry out the electrochemical etching: (a) $20 \times 20 \mathrm{~mm}^{2}$ silicon sample; (b) copper contact; (c) electrochemical cell; (d) platinum spiral. 


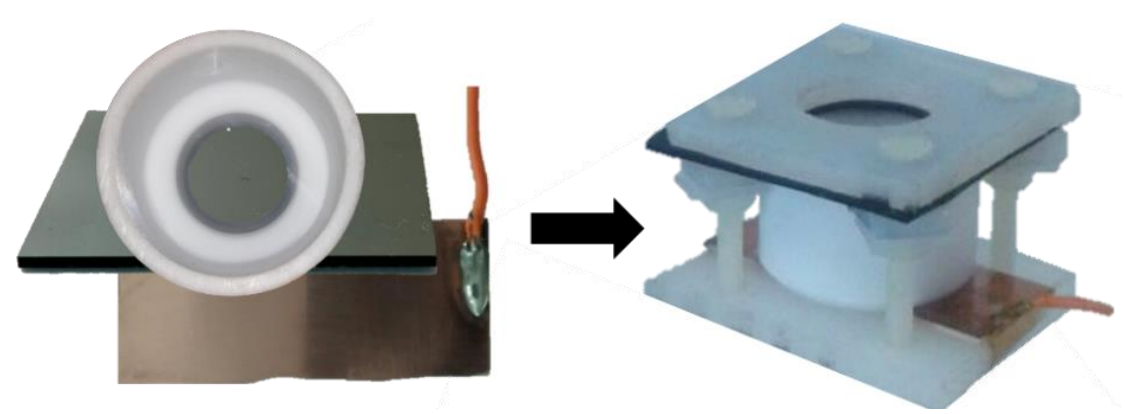

Fig. 3.7: Illustration of the electrochemical system building up.

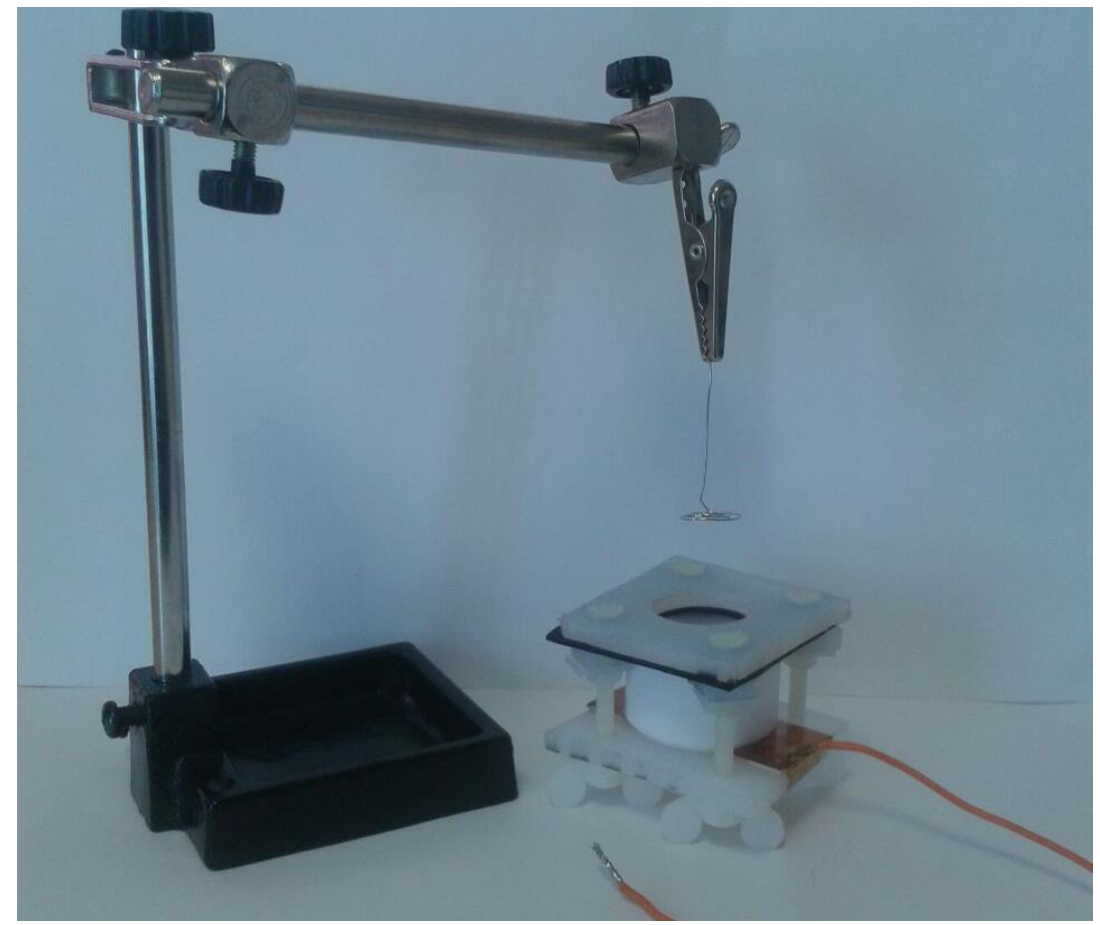

Fig. 3.8: Configuration used in the gas hood to perform the electrochemical etching.

\subsubsection{Anodization parameters}

The anodization parameters to be controlled for the fabrication of a PS multilayer with the required spectrum are the HF concentration, the current density and the etching time.

- HF concentration: It determines the pore diameter. With lower HF concentrations, it is possible to obtain higher pore diameters and vice versa. This means that, to obtain a porous layer with wide pores and high porosity it is not possible to use high HF concentrations and, thus, high current density neither and a balanced agreement between the two parameters must be reached. This parameter also determines the current density limit. With high HF concentrations, it is possible to work at high current densities. Instead, with low HF concentration, low current densities are needed in order to avoid electropolishing. 
- Current density: It is the main parameter that controls the porosity. Higher current densities lead to higher porosities and vice versa. It is important to control the highest value of this parameter because, if too high, electropolishing can occur. Furthermore, the current density affects the grown of the layer: with higher current densities, the PS layer grows more rapidly.

- Etching time: It controls the thickness of the layer. Since the growth of the layer depends also on the current density, the time of the process is selected taking it into account.

It has to point out the importance of a critical parameter to consider during the etching process: the growth rate. It depends mainly on the current density. However, the growth rate is not linearly dependent on the density current, since it depends also on the electrolyte composition.

Bisi et al. in [31] reported the effects of the anodization parameters on the PS formation, as shown in Fig. 3.9.

Effect of anodization parameters on PS formation

\begin{tabular}{llll}
\hline An increase of ... yields a & Porosity & Etching rate & Critical current \\
\hline HF concentration & Decreases (see text) & Decreases & Increases \\
Current density & Increases & Increases & - \\
Anodization time & Increases & Almost constant & - \\
Temperature & - & - & Increases \\
Wafer doping (p-type) & Decreases & Increases & Increases \\
Wafer doping (n-type) & Increases & Increases & - \\
\hline
\end{tabular}

Fig. 3.9: Effect the anodization parameters on the PS formation (Table from [31])

\subsubsection{Electrochemical etching}

In this work, our PS structures were fabricated by an electrochemical etching of a singleside, polished, boron-doped $<100\rangle$ oriented c-Si wafer, with an electrical resistivity of 0.01-0.02 $\Omega \cdot \mathrm{cm}$. Before the etching process, the silicon wafer was cleaned using a piranha solution (volume ratio $\mathrm{H}_{2} \mathrm{SO}_{4}: \mathrm{H}_{2} \mathrm{O}_{2}=3: 1$ ) at room temperature, in order to remove possible organic residues on the surface. Afterwards, the wafer was rinsed with deionized water (DIW).

In this $\mathrm{PhD}$ Thesis, the first etching parameter that we determined and then set as standard parameter for all the etching processes was the HF concentration. According to the HF concentration value, the etching parameters to be changed during the etching process were the current density as a function of the porosity of the layers. Since the current density affects the grown of the PS layer, the etching time was selected depending on the applied current density and the required thickness of the layers. 
Hence, the solution used to produce the PS was composed by a mixture of $49 \% \mathrm{HF}$ aqueous solution and $99 \%$ ethanol $(\mathrm{EtOH})$ solution, in a 1:2 relation. This leads to a $\mathrm{HF}$ concentration of $16 \%$. This concentration allowed us to obtain PS layers with RI between 1.3 and 2.6 refractive index unit (RIU), which represents a wide working range and allowed us to fabricate high RI contrast PS structures. The current density value was selected between 6 and $75 \mathrm{~mA}$.

During the etching, the electrochemical reaction takes place at the Si surface, where the dissolution of Si originates, as shown in Fig. 3.10. The Si surface is characterized by the presence $\mathrm{Si}-\mathrm{H}$ bonds. Due to the presence of a charge carrier at the Si surface, the Si-H bonds react with $\mathrm{F}^{-}$ ions present in the HF solution. The result is the formation of new Si-F bonds, which leads to the release of $\mathrm{Si}$ atoms from the Si surface.

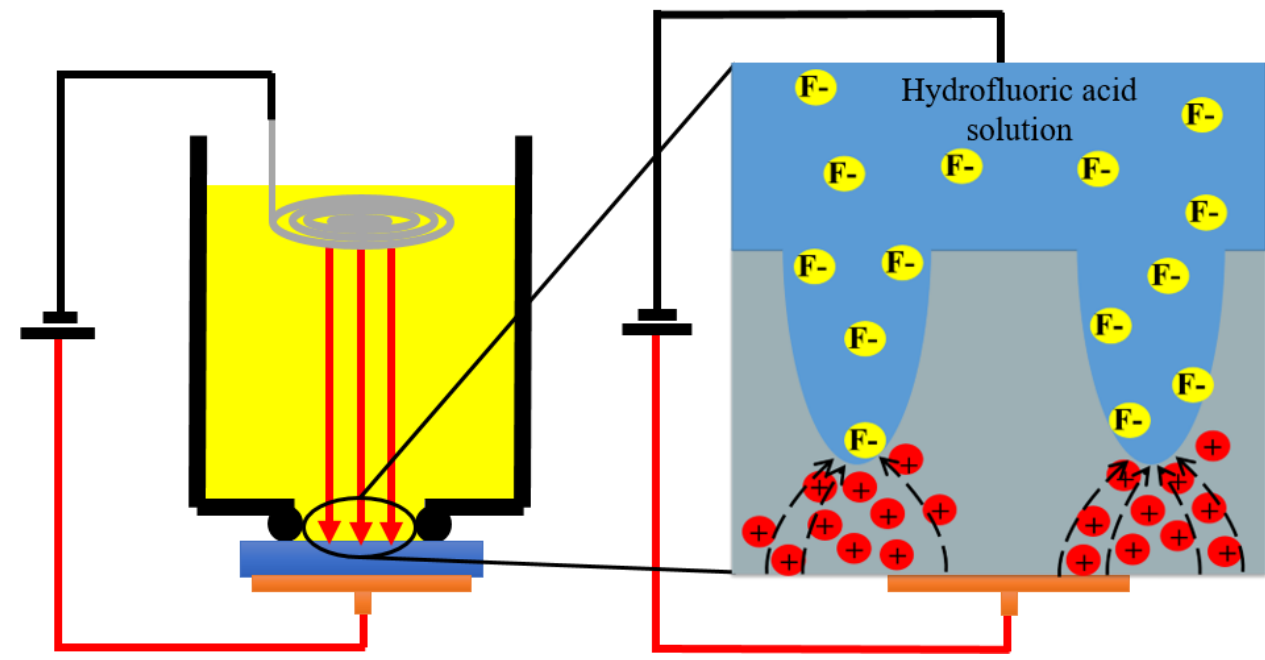

Fig. 3.10: Schematic illustration of the pores formation at the Si surface.

Korotcenkov explains the electrochemical etching of Si not as a result of only one chemical reaction, but as a result of the combination of four chemical reactions, all present during the process [79]:

- Direct dissolution of Si: $\mathrm{Si}+6 \mathrm{~F}^{-}+2 \mathrm{H}^{+}+2 h^{+} \rightarrow \mathrm{SiF}_{6}^{2-}+\mathrm{H}_{2}$

- Oxidation of $\mathrm{Si}: \mathrm{Si}+2 \mathrm{H}_{2} \mathrm{O}+4 h^{+} \rightarrow \mathrm{SiO}_{2}+4 \mathrm{H}^{+}+4 e^{-}$

- Oxide dissolution: $\mathrm{SiO}_{2}+6 \mathrm{~F}^{-}+6 \mathrm{H}^{+} \rightarrow \mathrm{H}_{2} \mathrm{SiF}_{6}+2 \mathrm{H}_{2} \mathrm{O}$

- Hydrogen termination of the surface: $\mathrm{Si}^{x}+x \mathrm{H}^{+} \rightarrow \mathrm{SiH}_{x}$

Concretely, the dissolution of $\mathrm{Si}$ can be originated by direct dissolution of $\mathrm{Si}$ or by oxidation of $\mathrm{Si}$ and oxide dissolution. 
The electrochemical etching process was always performed in a fume hood, in the clean room facility provided by the NTC, and at room temperature. In Fig. 3.11, the electrochemical system while performing the etching process is shown. Fig. 3.11 (a) shows a lateral view of the system, where the black alligator clip holds the platinum spiral. More in detail, in Fig. 3.11 (c), it is possible to appreciate the hydrogen bubbles going out from the PS structure formation. This confirmed us the effectiveness of ethanol in the HF solution to remove the hydrogen bubbles forming in the pores. Finally, the sample was cleaned with acetone, isopropyl alcohol and DIW to remove residual surface impurities.
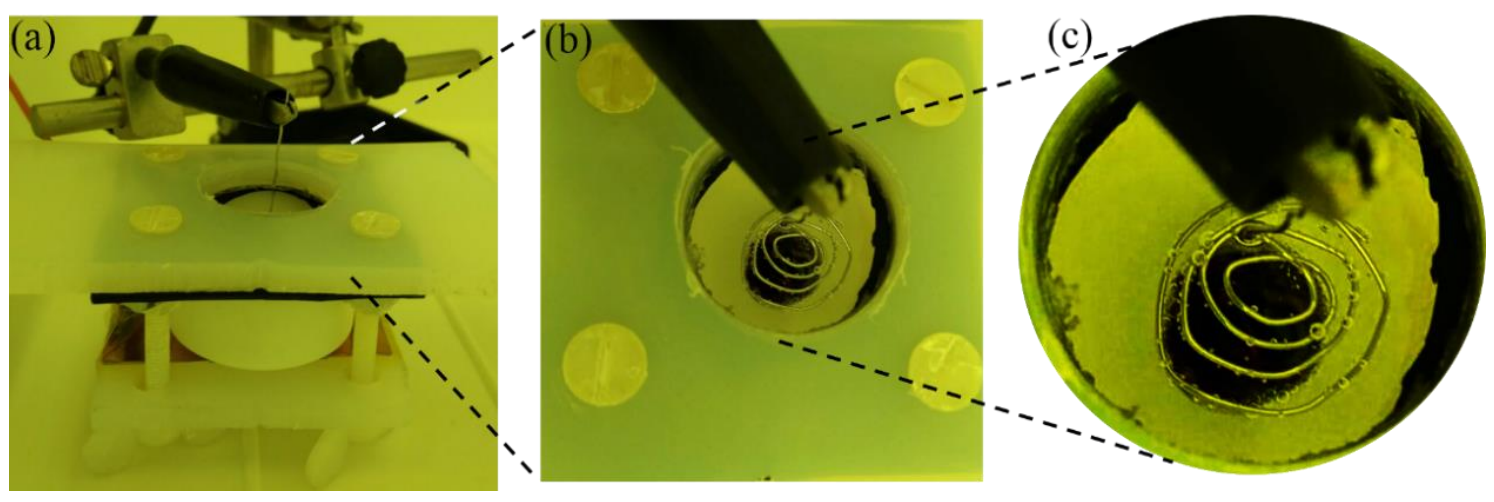

Fig. 3.11: Electrochemical system performing the etching process: (a) lateral view; (b) top-view; (c) top-view zoom focusing the platinum spiral immersed in the HF solution. The yellows light is due to the fact that the process was performed in the clean room, where such kind of light is used in order to avoid a alterations of the samples in lithographic processes.

Figure 3.12 shows several PS samples fabricated with different current densities. When using very low current densities, close to the lower limit, the pores formation can take place only in some regions of the etched area, as it can be observed in the first PS sample. By increasing the current density, the etched area is darker, due to the increase of porosity. When using current densities included between the lower limit and intermediate values, the porous region uses to be uniform. When using very high current densities, close to the higher limit, we observed a lack of uniformity in porosity and in layer thickness.

The etched area used to be characterized by a uniform region, usually located close to the PS-silicon border, and a non-uniform region comprised by several circumferences. This was due to the shape of the platinum spiral. We observed that the spiral shape began to affect to the uniformity of the porosity and of the thickness of the PS structures at limit current density values. 

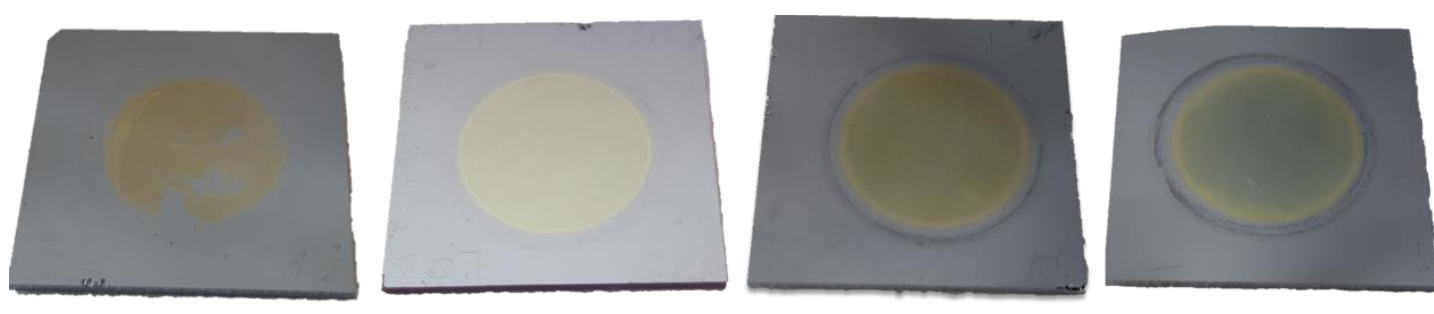

CURRENT DENSITY
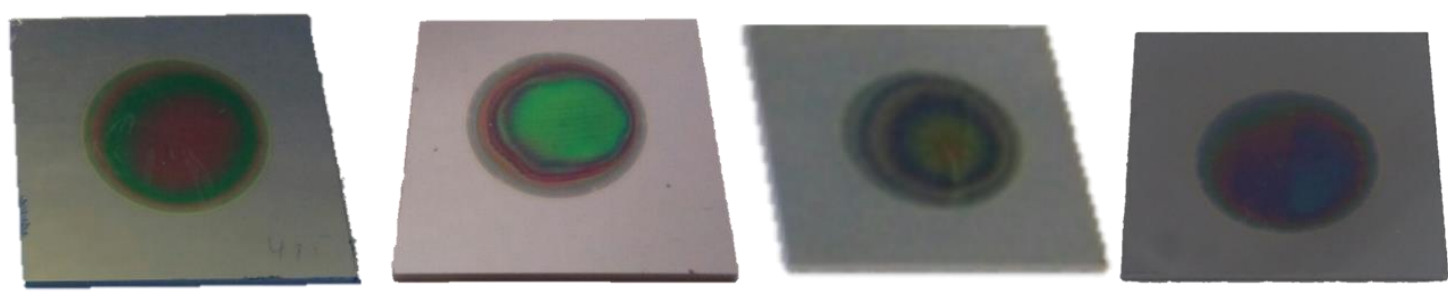

\section{CURRENT DENSITY}

Fig. 3.12: PS samples fabricated with different current densities.

Figure 3.13 shows a PS sample fabricated using an intermediate current density. The zoom shows a field emission SEM (FE-SEM) image of the structure surface. The $16 \% \mathrm{HF}$ concentration determined an average porous diameter in the range of $10 \mathrm{~nm}$. To obtain this value, the ImajeJ software was used [80].

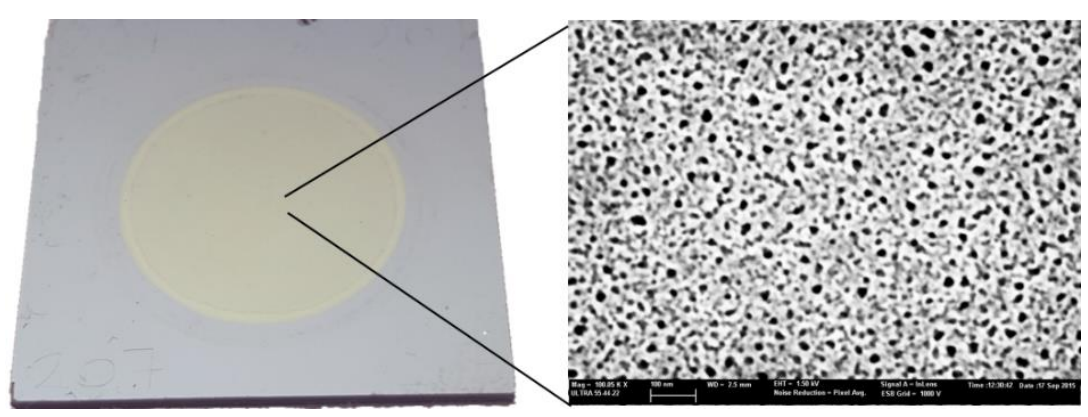

Fig. 3.13: Picture of a silicon sample after the electrochemical etching. The inset shows a FE-SEM image of the porous structure surface.

\subsubsection{Oxidation}

After the electrochemical etching process, PS presents a surface characterized by $\mathrm{Si}-\mathrm{H}$ groups, with the hydrogen atoms exposed toward the environment medium. This makes PS hydrophobic. Fig. 3.14 shows an example of a hydrophobic PS sample. This sample was just fabricated. After the in-air characterization process, the sample had to be characterized in water. So, the box containing the PS sample was filled with the mentioned liquid. In these images, it is possible to appreciate the water wall formed when water reached the PS region due to the PS hydrophobicity. 

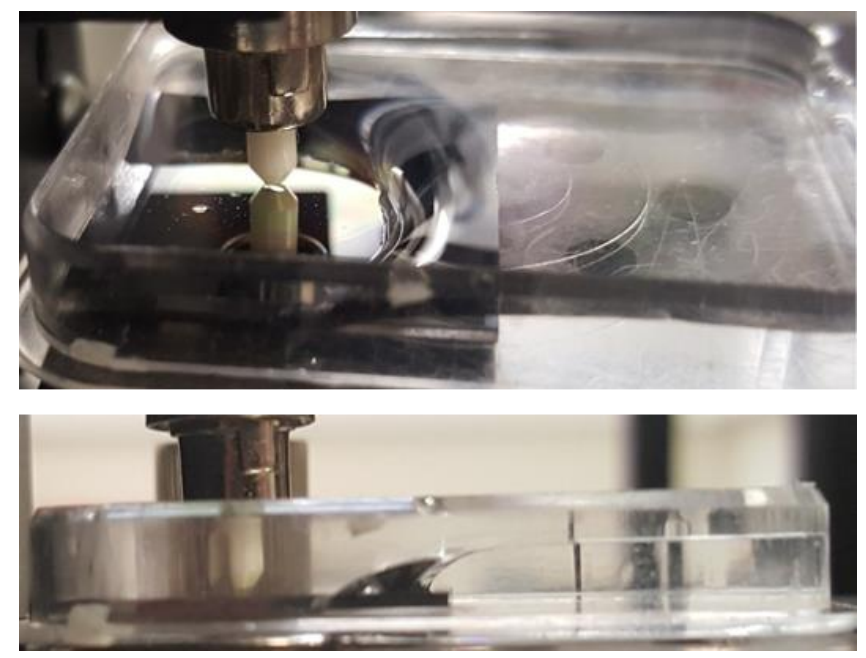

Fig. 3.14: Pictures showing the hydrophobicity of a PS sample when in contact with water.

In conclusion, PS hydrophobicity avoids water to fill the pores. This represents a considerable limitation for sensing purposes, since the solution transporting the target analyte cannot get into the pores. In order to overcome such limitation, a subsequent process was considered in order to make the PS hydrophilic: PS oxidation. As a matter of fact, the PS oxidization gives rise to a new $\mathrm{SiO}_{2}$ layer, thus making the surface hydrophilic.

Moreover, PS oxidization was applied to stabilize the PS structure. After the electrochemical etching, the PS surface is highly reactive. The new layer of oxide passivates the surface, avoiding its over time modification. For this reason, in this work, after electrochemical etching, the PS samples were oxidized. The oxidation was obtained with a 10 minutes oxygen plasma treatment carried out with a plasma asher. Figure 3.15 shows the same PS sample in contact with water, before and after the oxidization process. It is possible to observe the hydrophilicity of the sample after the oxidation.
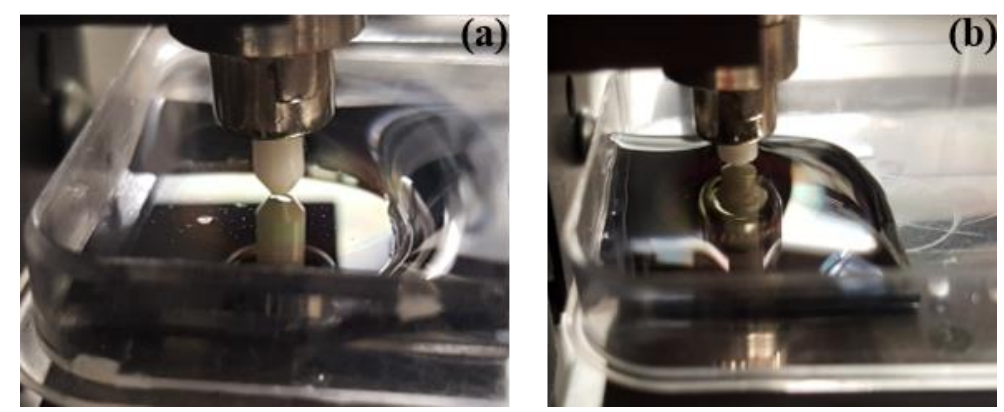

Fig. 3.15: Picture of a PS sample in contact with water (a) before and (b) after the oxidization. 


\subsection{Experimental opto-fluidic setup}

Our objective in this work was to carry out a continuous monitoring of the PSMC sensing response in real-time, which would allow us to detect extremely small RI variations occurring on the sensing structure. Typically, most works focused on the development of PSbased sensors only perform a measurement before and after the target substance was deposited on the sensing structure [36]. To do this, the sensing sample was usually removed from the experimental setup and then re-placed, leading to a significant error in the measurement, and thus limiting the experimental accuracy, especially when very small spectral shifts needed to be measured. In order to overcome this problem and to remove that source of inaccuracy, a flow cell was used to flow the substances under analysis, without removing the sample from the experimental setup [74]. This also allowed us to monitor the evolution of the sensing signal while the substance was flowing, in order to determine the kinetics of the sensing events being monitored, which also leads to an improvement of the sensing performance. On the other hand, the characterization equipment that is typically used provides a very poor spectral resolution and can require a very long time to acquire each spectrum. This makes it difficult to detect very small spectral shifts with high accuracy and to obtain a real-time monitoring of the sensing events.

In order to overcome these limitations, we developed an opto-fluidic experimental setup, which was able to carry out the sensing measurements in-continuum, in real-time, and with a high spectral resolution. The setup was composed of fluidic and optical parts operating at the same time, in order to flow the reagents over the sample and simultaneously monitor the reflectivity spectrum of the PS structure.

In order to characterize the reflectivity spectrum, the PS sample was illuminated with an optical fiber perpendicularly placed on top of the PS surface. To this aim, the central element of the experimental setup was a homemade fluidic cell. This cell allowed us to flow over the PSMC sample different solutions while it was simultaneously illuminated with the perpendicularly placed optical fiber, as shown in Fig. 3.16. The fluidic cell was basically composed of a $4 \times 4 \mathrm{~cm}$ polymethyl methacrylate (PMMA) base, a Viton channel and an upper PMMA piece. The PMMA base was the support on which the PS sample was placed. The Viton channel was placed on top of the PS sample, in order to flow the target substance only over the region where the optical interrogation was carried out. The upper PMMA piece was placed on top of the PS-Viton channel system to seal the fluidic channel. In the upper PMMA piece, the input and output fluidic tubes and the optical fiber were attached. Some pressure was applied to the upper PMMA piece to hermetically seal the fluidic system. To reduce the possibility of breaking the PS structure, a polydimethylsiloxane (PDMS) base was placed under the PS sample to absorb the pressure. Furthermore, the PDMS base attenuated any possible external vibrations. 

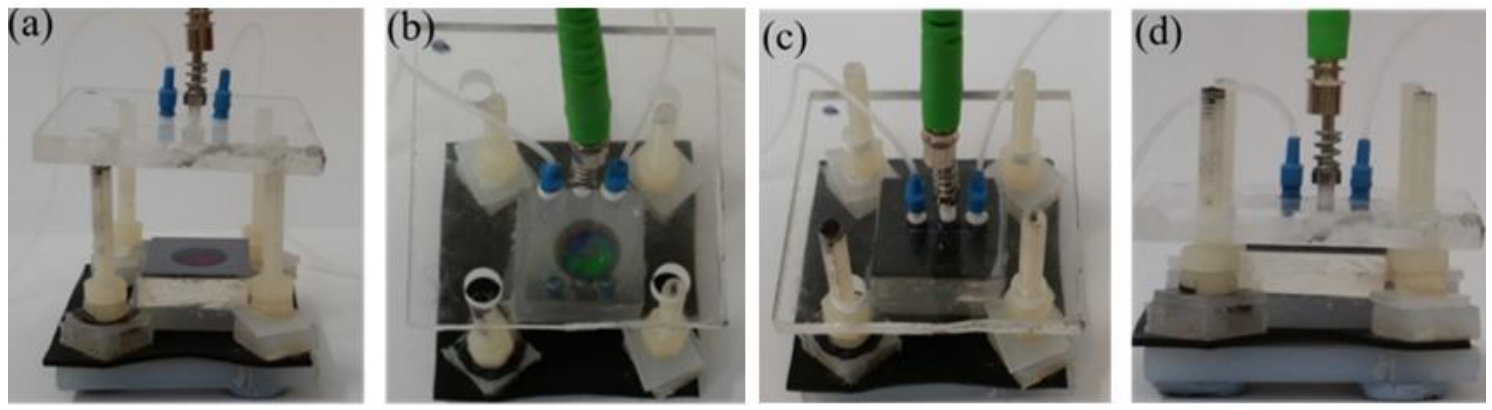

Fig. 3.16: Pictures of the opto-fluidic experimental cell used to flow the solutions over the PS sample: (a) lateral view open cell; (b) top view open cell; (c) top view hermetically sealed cell; (d) lateral view hermetically sealed cell.

The optical fiber was connected to an optical interrogator (OI) (PXIe-4844 National Instruments). The OI is an optical measuring apparatus able to obtain one spectrum every $0.1 \mathrm{~s}$ in a wavelength range of 1510-1590 $\mathrm{nm}$ with a resolution of $4 \mathrm{pm}$. This apparatus provides the advantage of analysing the reflected light spectrum in real-time with a very high resolution. A LabVIEW program was implemented to continuously examine the evolution of the reflectance spectrum.

When the experiments were carried out, the solutions were flowed using the syringe pump introduced in chapter 2. The whole experimental opto-fluidic setup is shown in Fig. 3.17.

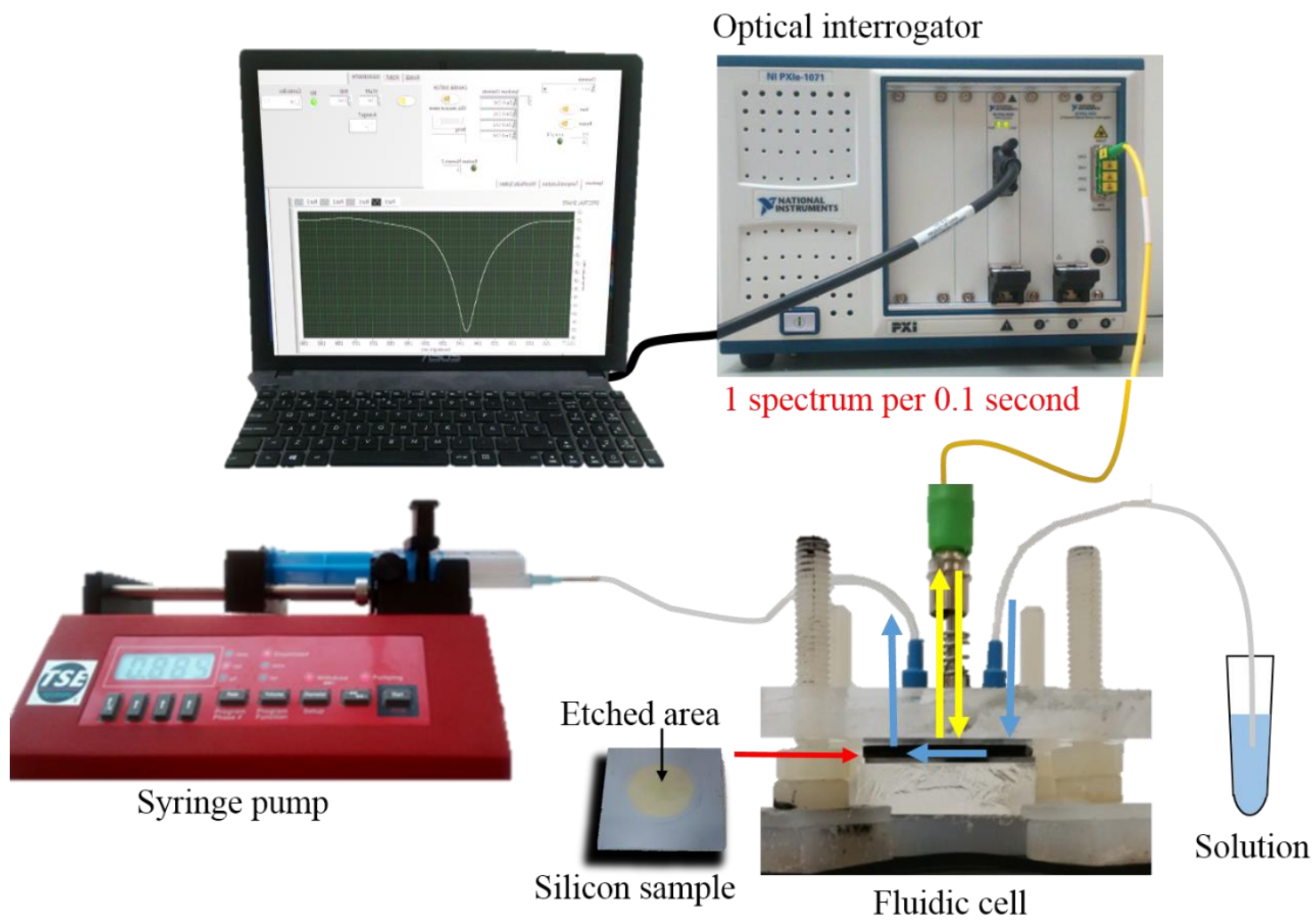

Fig. 3.17: Schematic illustration of the opto-fluidic setup used to carry out the sensing experiments 


\subsection{Sensing using Bragg reflectors}

\subsubsection{Power-based sensing technique}

Initially, the technique used to carry out the measurements is based on monitoring the evolution of the integral relative to a selected interval of the reflected band provided by the PS structure averaged with the interval itself. The sensing technique is schematically illustrated in Fig. 3.18. At the initial state, a certain amount of interval integral is measured. Then, any change in the refractive index produced in the sensing structure either by a molecular recognition event or by bulk refractive index variation leads to a shift of the reflected band position. This shift is translated into a variation of the interval integral. Hence, in our experiments, by monitoring the interval integral, we were able to perform an indirect measurement of the PBG position and subsequently to obtain the sensing information.

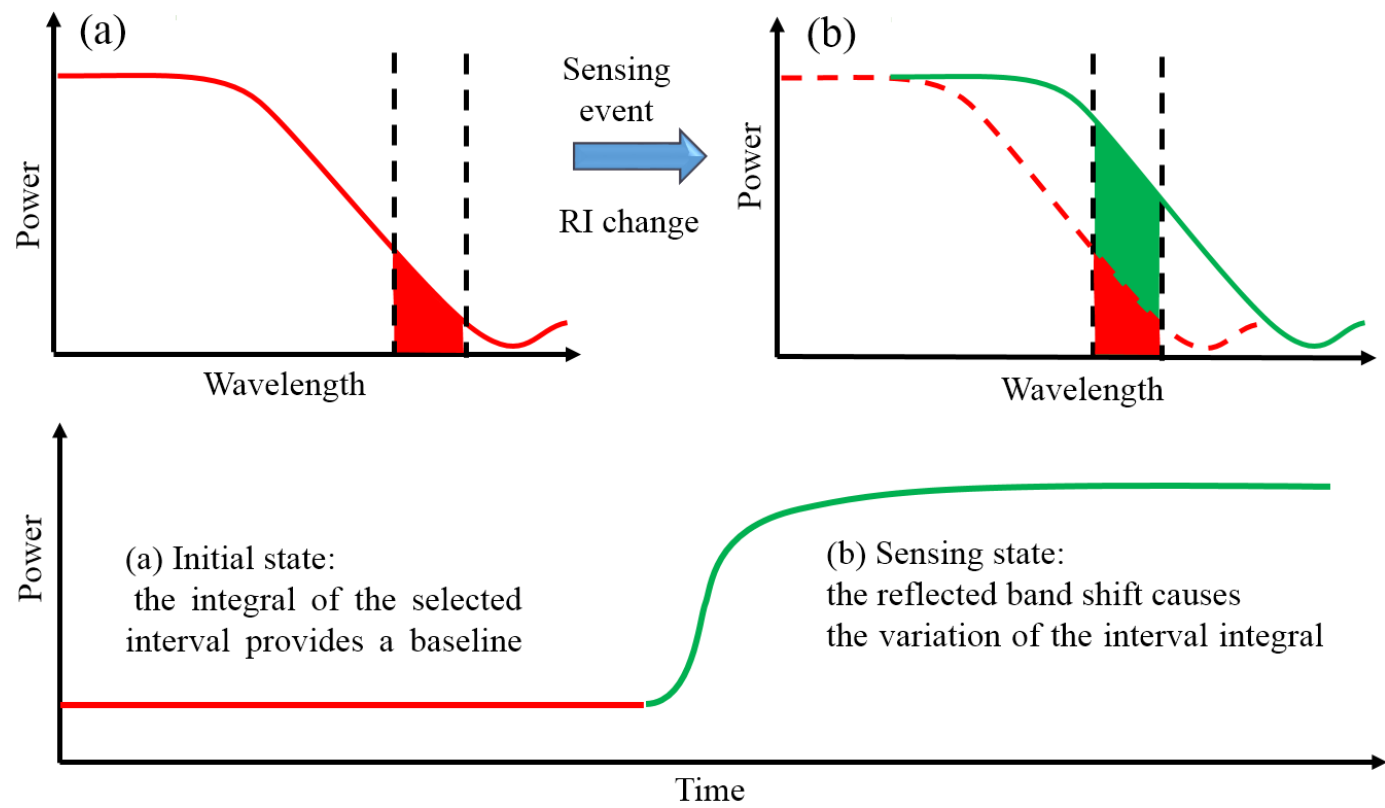

Fig. 3.18: Schematic representation of the power-based sensing technique. When the reflected band edge of the structure is shifted, the integral of the selected interval changes.

\subsubsection{Experimental results}

\subsubsection{1 $1^{\text {st }}$ experiment}

The Bragg reflector design was aimed at locating the right edge of the reflectance band in the spectral range available from the optical interrogator when considering water environment. To this aim, the designed multilayer structure was composed of 18 periods where the RI of the low and high porosity layers were, respectively, $n_{H}=1.84$ and $n_{L}=1.6$ and the corresponding thicknesses were $d_{H}=170 \mathrm{~nm}$ and $d_{L}=200 \mathrm{~nm}$. Figure 3.19 shows the calculated reflectance spectrum of the designed structure. 


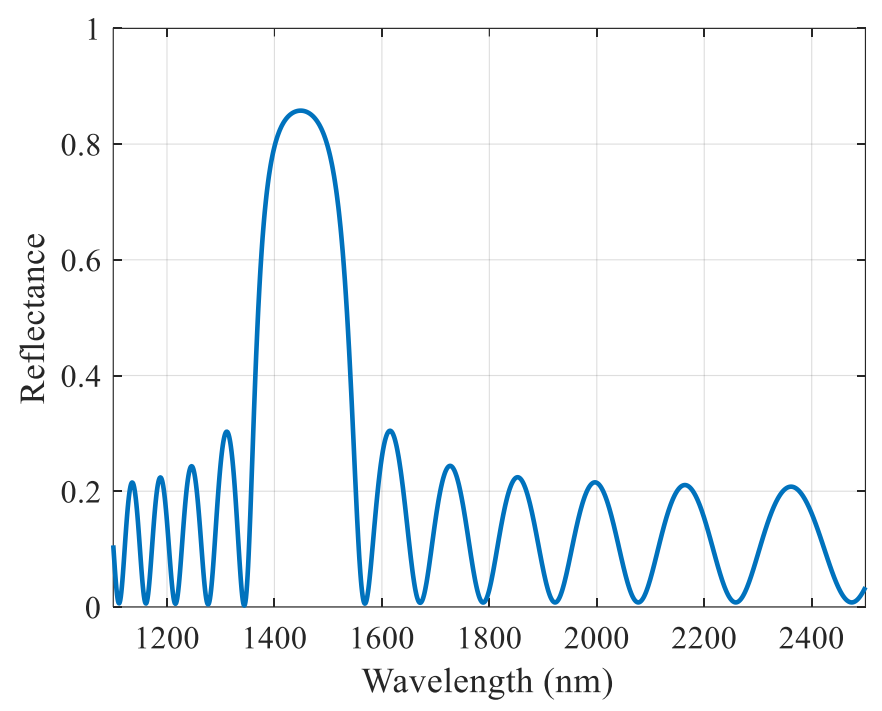

Fig. 3.19: Simulated spectrum of the designed Bragg reflector.

The current densities and the etching time used to create the $n_{H}$ and $n_{L}$ layers were 12 mA, 35 mA, 5.8 seconds and 10.2 seconds. Figure 3.20 shows a SEM cross-sectional image of the multilayer structure, where the light and dark grey layers are the $n_{H}$ and the $n_{L}$ layers, respectively. The average measured thicknesses of both layers are 150 and $190 \mathrm{~nm}$, respectively.

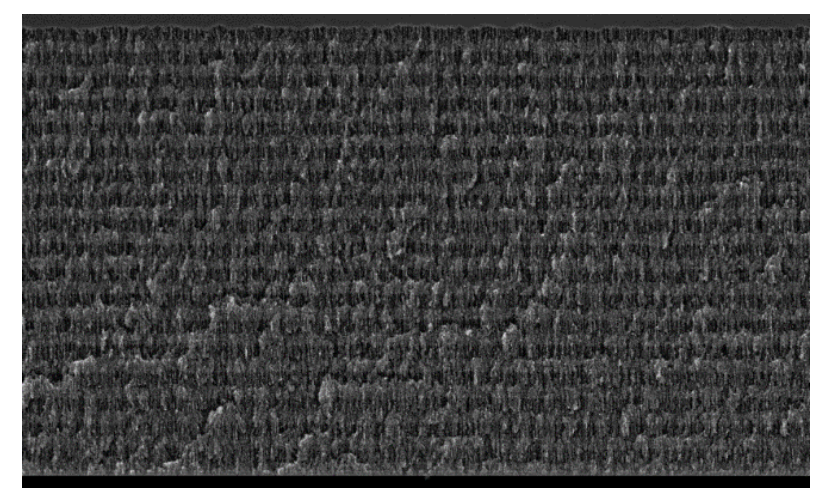

Fig. 3.20: SEM cross-sectional image of the fabricated PS multilayer structure.

Once the sample was located in the fluidic cell and DIW started to flow, the LabVIEW application was used to show and monitor the PS structure reflectance spectrum measured with the OI. Figure 3.21 shows the interface of the LabVIEW application used to monitor the reflectance spectrum evolution, whereas Fig. 3.22 shows the reflectance spectrum measured with the OI. The interval integral selected to be monitored during the experiment was $1580-1585 \mathrm{~nm}$. In this interval, a slope of $\sim-0.4 \mathrm{~dB} / \mathrm{nm}$ was determined. 


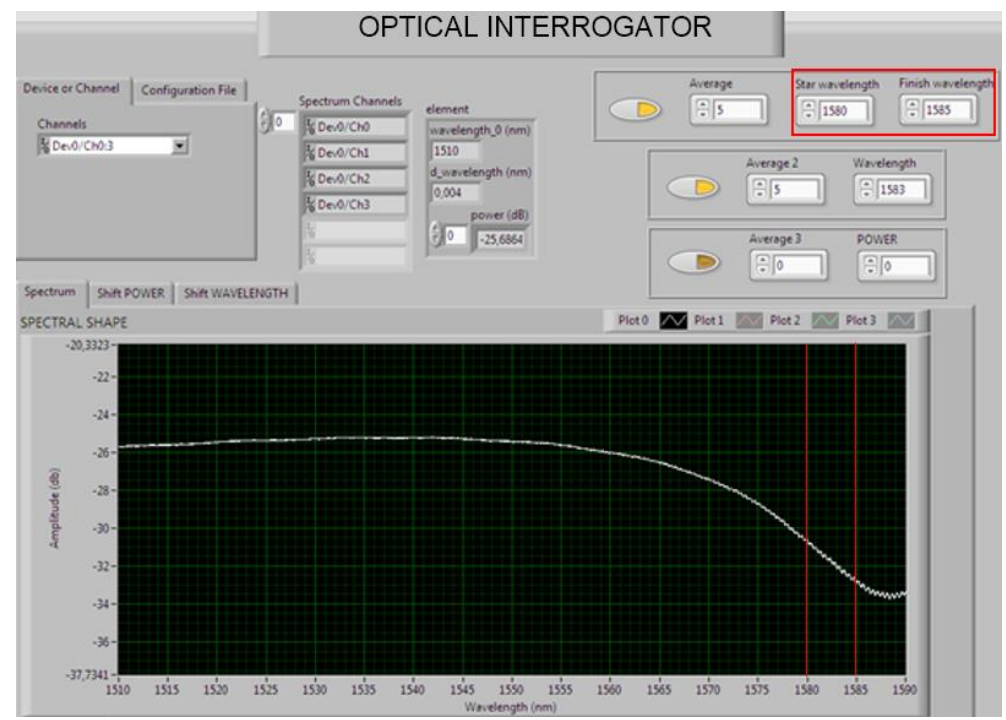

Fig. 3.21: LabVIEW application interface used to monitor the reflectance spectrum.

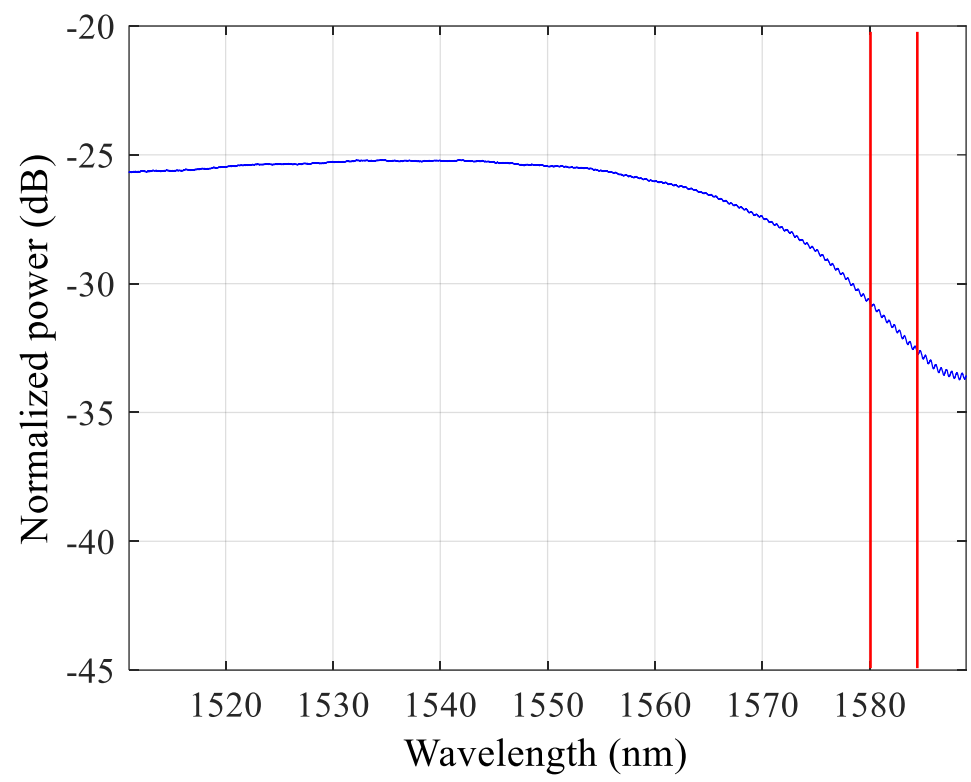

Fig. 3.22: Reflectance spectrum measured with the OI.

The experimental sensing procedure consisted in flowing several EtOH dilutions in DIW over the PS multilayer and monitoring in real-time the evolution of the spectrum when the RI was changed. Since our working conditions were $\lambda=1550 \mathrm{~nm}$ and $\mathrm{T}=25^{\circ} \mathrm{C}$, the RI value of water considered for the experiments was 1.3173, obtained from [81]. The EtOH concentrations in DIW were $5 \%$ and $10 \%$, which means a relative RI change of $6.6 \cdot 10^{-3}$ and $3.3 \cdot 10^{-3}$ RIU, respectively. Such RI values were obtained by using the model proposed in [81]. The power time evolution of the selected interval is shown in Fig. 3.23. Variations of $\sim 0.42 \mathrm{~dB}$ and $\sim 0.84 \mathrm{~dB}$ were observed for EtOH 5\% and 10\%, respectively. This led to a sensitivity of around $127 \mathrm{~dB} / \mathrm{RIU}$. A noise of $8 \cdot 10^{-4} \mathrm{~dB}$ was observed, what led to a limit of detection of $6.29 \cdot 10^{-6} \mathrm{RIU}$. 


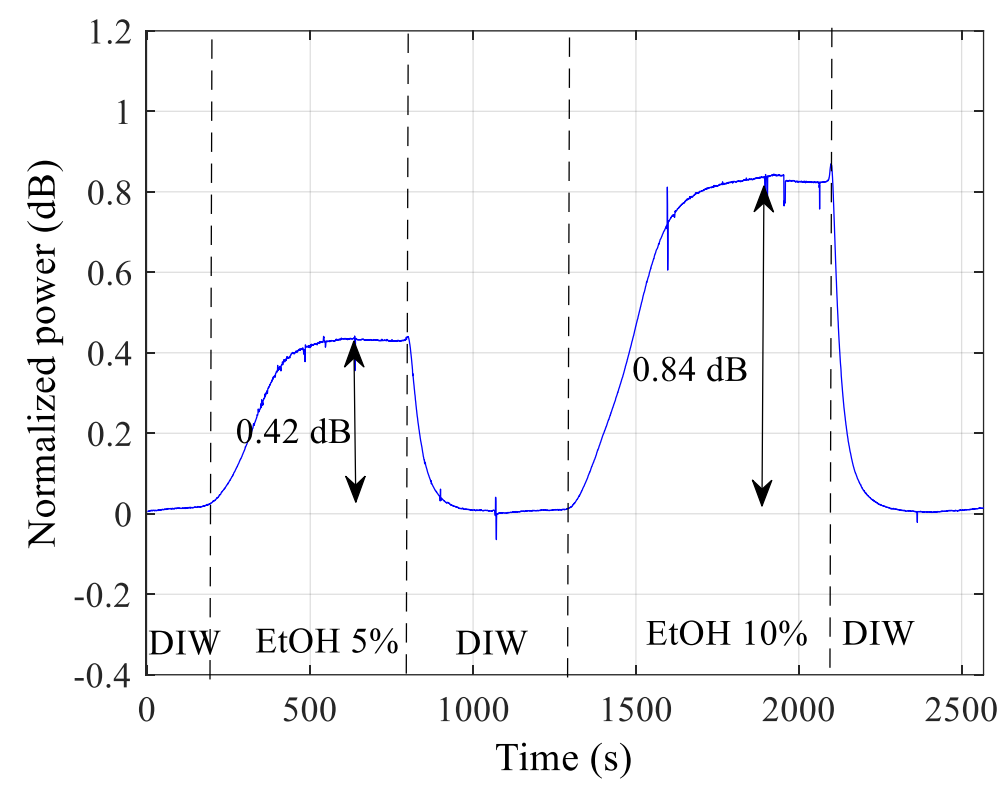

Fig. 3.23: Power time evolution of the first experiment.

A first deduction from the analysis of these results was the dependence of the power sensitivity on the spectrum slope in the selected interval. The sharper was the slope the higher the power sensitivity would be. For this reason, a method to improve the power sensitivity became the new objective.

\subsubsection{2nd experiment}

To confirm what deducted in the previous experiment, another experiment was carried out by employing another PS sample. This time, the structure design was aimed at locating one of the left lobes besides the reflected band in our measurement spectral range. The reason why we considered a left lobe is due to the fact that, in that region, the lobes are narrower and thus they present a higher slope (which is even higher as we move along those lobes). The designed multilayer structure was composed of 10 periods. The RI of the low and high porosity layers were, respectively, $n_{H}=2$ and $n_{L}=1.56$ and the corresponding thicknesses were $d_{H}=250 \mathrm{~nm}$ and $d_{L}=320$ $\mathrm{nm}$. Figure 3.24 shows the calculated reflectance spectrum of the designed structure. 


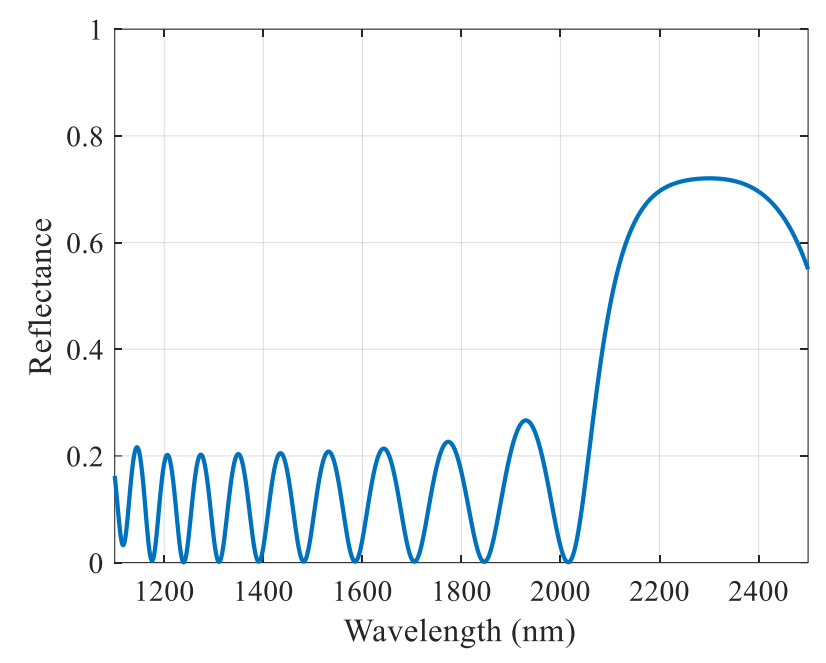

Fig. 3.24: Simulated spectrum of the designed Bragg reflector.

The current densities and the etching times used to create the $n_{H}$ and $n_{L}$ layers were 12 mA, $33 \mathrm{~mA}, 21.3$ seconds and 13.3 seconds. Figure 3.25 shows a SEM cross-sectional image of the multilayer structure, where the light and dark grey layers are the $n_{H}$ and the $n_{L}$ layers, respectively. The average measured thicknesses of both layers are 150 and $190 \mathrm{~nm}$, respectively.

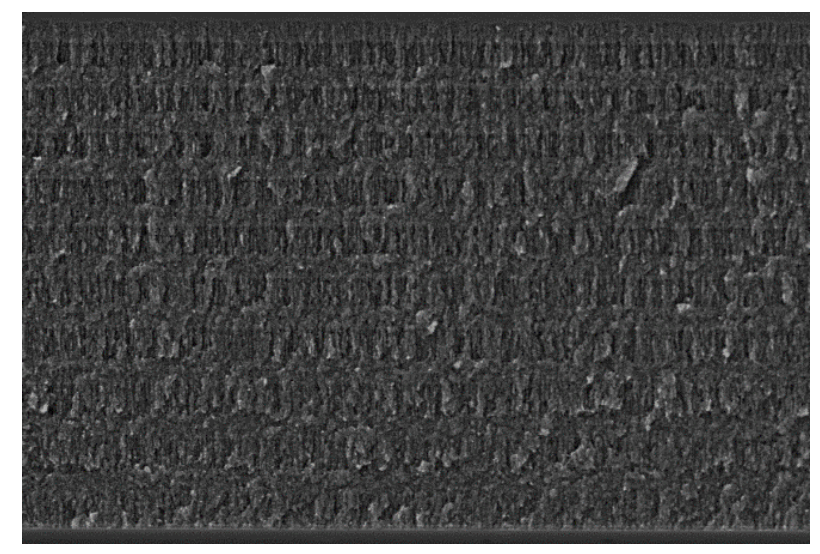

Fig. 3.25: SEM cross-sectional image of the second fabricated Bragg reflector.

In Fig. 3.26 (a), the reflectance spectrum measured with the OI is shown. It is possible to appreciate the presence of a drop of almost $20 \mathrm{~dB}$ in the interval $1570-1585 \mathrm{~nm}$, with a slope of $\sim-1.4 \mathrm{~dB} / \mathrm{nm}$. So, to carry out the power based sensing technique, in this range, two different intervals were selected: 1580-1583 and 1583-1586. The reflectance spectrum also provided a Lorentzian shape in the interval 1510-1540 $\mathrm{nm}$. So, besides the power based interrogation technique, also the position of the minimum in the spectra was tracked. In order to improve the precision of the minimum tracking, the monitored spectrum was filtered using the FFT algorithm and eliminating high frequency components. The filtered spectrum is shown in Fig. 3.26 (b). 

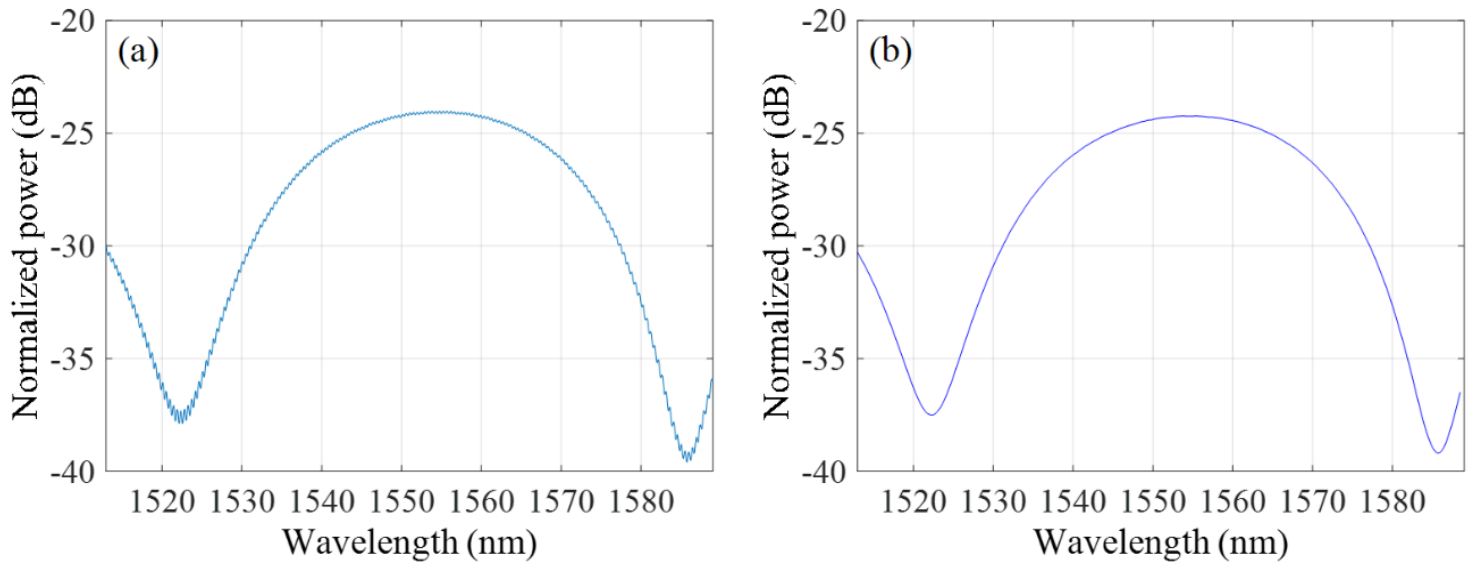

Fig. 3.26: Reflectance spectrum measured with the OI: (a) original; (b) FFT filtered.

Figure 3.27 shows the time evolution of the integrated power in the selected intervals. As it can be clearly observed, the power increase was not the same for both intervals. In the interval 1583-1586, the power increase was considerably higher than that relative to the interval 1580-1583. Furthermore, the power increase was not the linearly proportional to the EtOH concentration increase. In Fig. 3.28, it can be observed that the power sensitivity was not linear, confirming the previous observation. These issues are related with the different slope of the reflection spectrum as we move closer to the position of the minimum. The highest power increase was obtained in the interval 1583-1586 for EtOH 10\% and reached a value of $4 \mathrm{~dB}$. This means that a power sensitivity values around $606 \mathrm{~dB} / \mathrm{RIU}$ and a limit of detection of $\sim 1.32 \cdot 10^{-6} \mathrm{RIU}$ can be achieved.

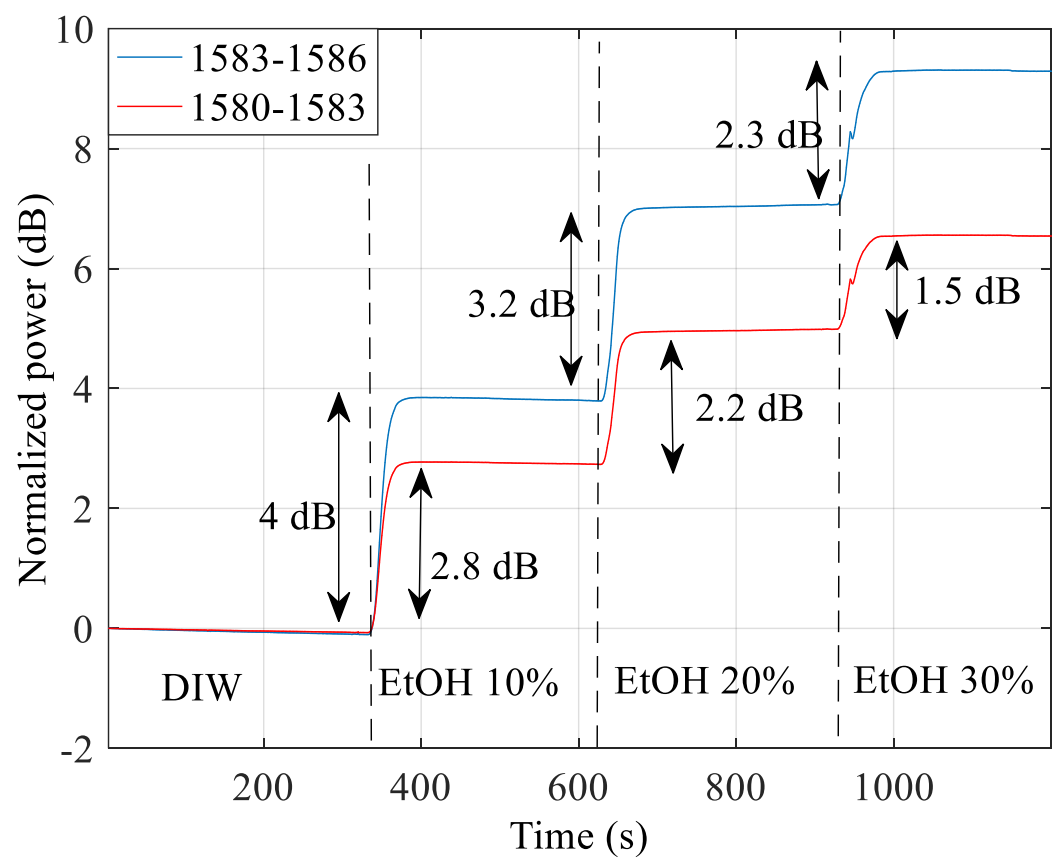

Fig. 3.27: Time evolutions of the integrated power of the selected intervals. 


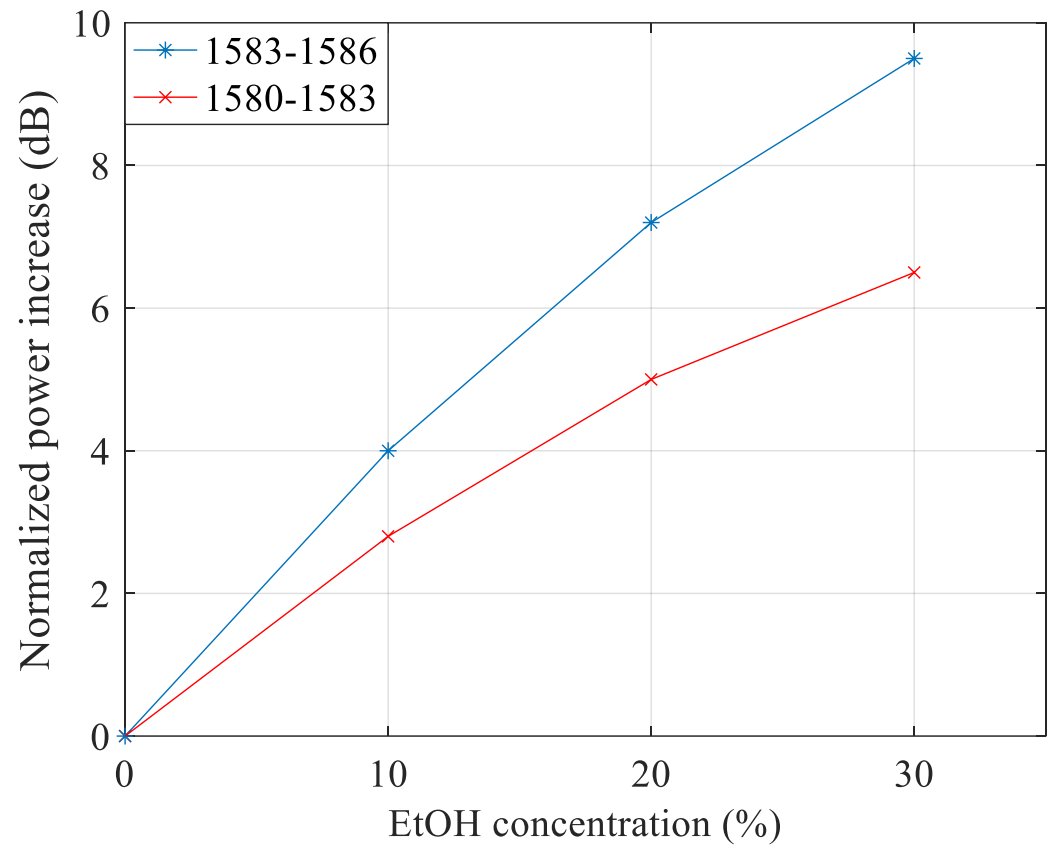

Fig. 3.28: Sensitivity curve of the integrated power of the selected intervals.

Figure 3.29 shows the time evolution of the wavelength shift. As it can be clearly observed, the wavelength shift was linearly proportional to the EtOH concentration increase. This means that the wavelength sensitivity was linear.

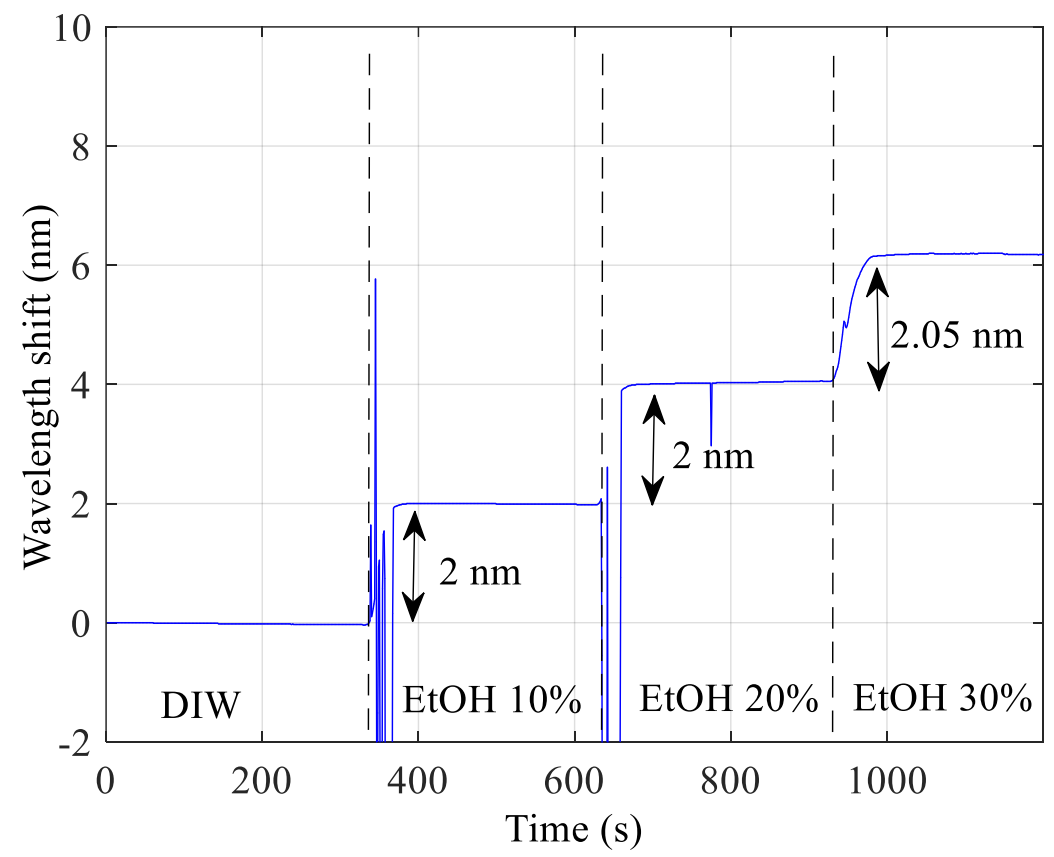

Fig. 3.29: Wavelength shift time evolutions of the second experiment.

To confirm the linearity of the wavelength shift also for low RI changes, EtOH 1\% was flowed and a wavelength shift of 200 pm was observed, as shown in Fig. 3.30. This confirmed the linear sensitivity also for low RI changes. 


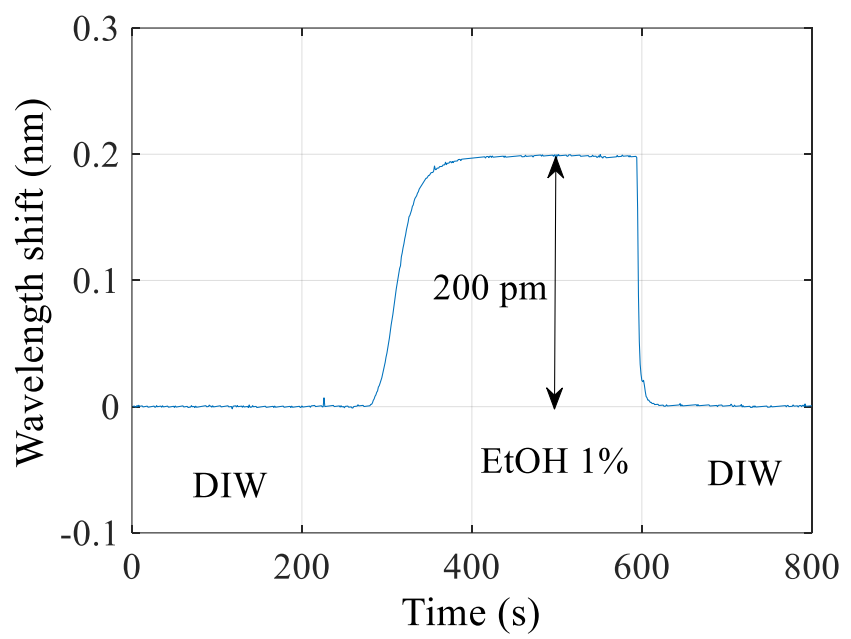

Fig. 3.30: Wavelength shift time evolution relative to EtOH $1 \%$.

\subsubsection{Results analysis}

The power increase was not the same for both the selected intervals since, in each interval, the spectrum was characterized by a different slope, as shown in Fig. 3.31 (a). The interval 1583-1586 was sharper. The non-linearity of the power sensitivity as a function of the EtOH concentration was due to the shift of the spectrum during the experiment, as shown in shown in Fig. 3.31 (b). For each 10\% increment of EtOH concentration, a wavelength shift of 2 nm was observed. This means that, over time, in the same interval, the spectrum slope decreased. Consequently, a decrease of the power sensitivity was observed.

In order to compare these results with those of the previous experiment, we considered the power evolution corresponding to EtOH $10 \%$. The power increase reached a value of $4 \mathrm{~dB}, 5$ fold the observed in the previous experiment. This higher sensitivity was due to the sharper spectrum slope than that of the previous experiment.
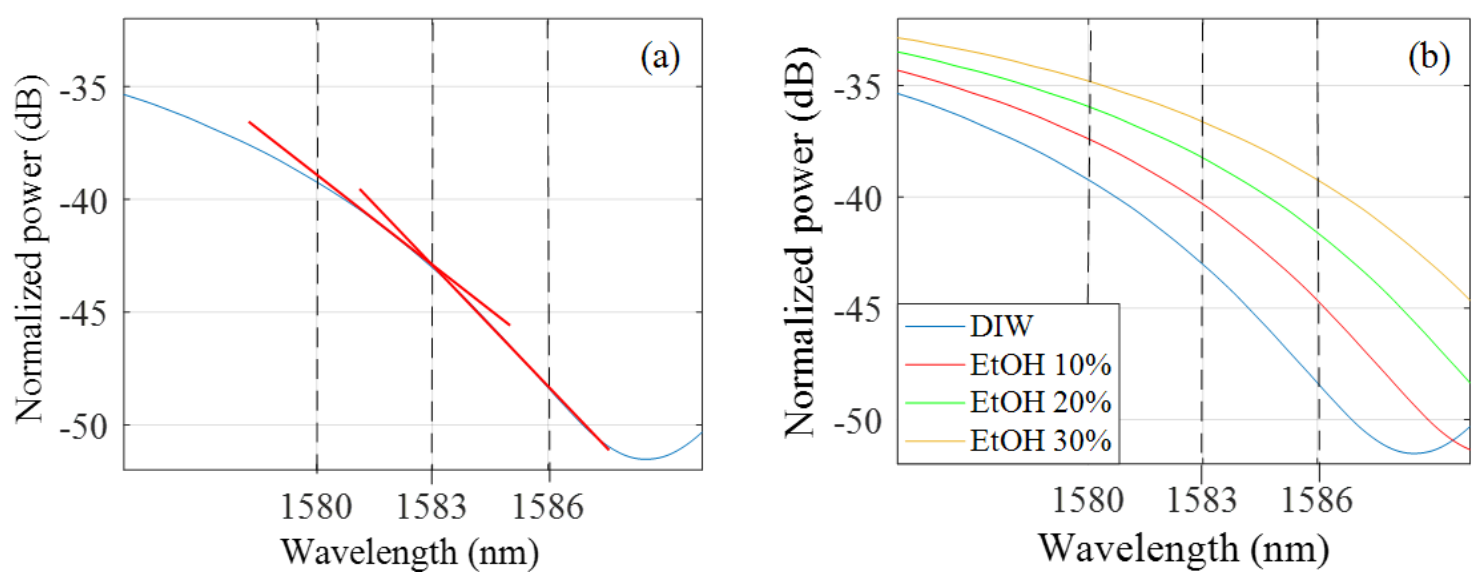

Fig. 3.31: (a) Reflectance spectrum in the selected intervals: the red lines represent the slopes. (b) Reflectance spectrum shifted of 2, 4 and $6 \mathrm{~nm}$. 


\subsubsection{Conclusions}

We learnt that the sensing technique based on the tracking of the power of a selected interval is a simple, rapid and powerful tool to reach high sensitivities and detect very low RI changes. However, the power sensitivity obtained by using this technique is not always linearly proportional to the RI variation of the solution filling the pores. This is due to the fact that the sensitivity strongly depends on the spectrum slope of the selected interval. In fact, the slope can change during an experiment owing to the spectrum shift caused by the RI change. Hence, when monitoring the power evolution, it is fundamental to take into account the shift of the spectrum during the experiment in order to obtain reliable results.

On the contrary, the wavelength shift was linearly dependent with the EtOH concentration and, therefore, the sensitivity was always the same for all the RI changes. In this case, the sensitivity does not depend on the sensing technique, but just on the structure itself. In conclusion, the monitoring of the wavelength of a spectrum minimum proved, once again, to be a suitable method for RI sensing.

\subsection{Experimental sensing using a PSMC}

The conclusions obtained in the previous section led us to perform the experiments monitoring the wavelength shift of the spectrum. For this reason, we focused our study on the development of a PSMC since it provides a sharp peak in the reflectance band that is the most suitable spectral characteristic to monitor, like the peak of a resonance of a ring resonator.

The aim was to achieve a structure as sensitive as possible. So, in order to enhance the sensitivity of the structure, high porosities of the layers are required, as this increases the total volume where the target substance is infiltrated. On the other hand, having an extremely high porosity of the layers will make the structure more fragile and easily collapsible. Therefore, we selected a maximum porosity value for the layers of $85 \%$ in order to have an as-high-as-possible sensitivity while keeping the structure robust.

\subsubsection{Experimental results}

The sequence $\left[n_{H}, n_{L}\right] \times 7, n_{H}, n_{L}-M C, n_{H},\left[n_{L}, n_{H}\right] \times 7$ was considered for the PSMC structure, as schematically depicted in Fig. 3.32 (a). In order to obtain a highly sensitive PSMC, we considered limit porosity values, which are $67 \%$ and $85 \%$. These porosities corresponded to RIs of $n_{H}=1.64, n_{L}=1.22$. Figure 3.32 (b) shows an example of the resonance peak shift evolution due to the infiltration of water into the pores of the structures. This evolution was taken into 
account so that the resonance peak position was located in the spectral range available from the optical interrogator when considering the pores filled by water.
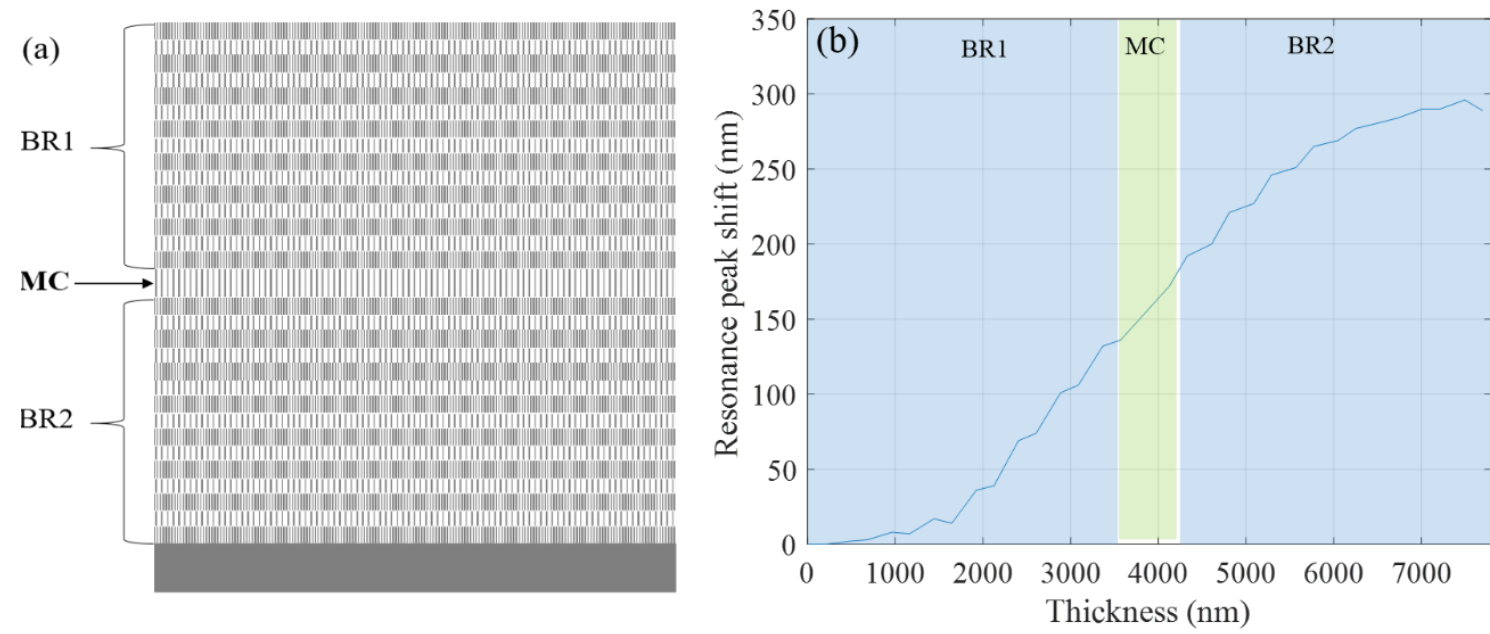

Fig. 3.32: (a) Scheme of the PSMC design; (b) example of the resonance peak shift evolution due to the infiltration of water into the pores of the structures

Since we considered limit porosity values, limit current densities were applied. In this case, unlike with the previous PS structures, a lack of uniformity was observed after the PSMC fabrication, as shown in Fig. 3.33. The etched area used to be characterized by a uniform region, usually located close to the PS-silicon border, and a non-uniform region comprised by several circumferences. This was due to the shape of the platinum spiral. We observed that the spiral shape began to affect to the uniformity of the porosity and of the thickness of the PS structures at limit current density values.

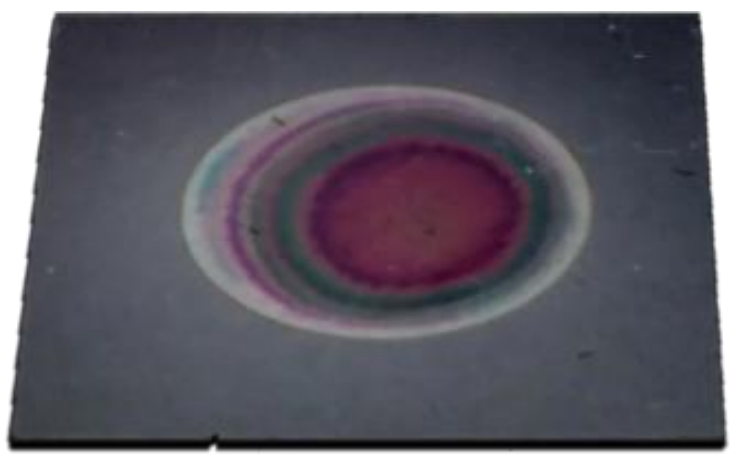

Fig. 3.33: Picture of a fabricated PSMC with lack of uniformity.

We observed also that, in the uniform region, the reflectance spectrum complied with the requirements, whereas, the lack of uniformity caused a 100-150 nm blue-shift of the resonance peak in comparison with the uniform region. So, we took also this issue into account in order to define the resonance peak position. For this reason, we considered a structure with the peak at 
$1450 \mathrm{~nm}$ in air. To this aim, the selected thicknesses of the layers were $d_{H}=220 \mathrm{~nm}$ and $d_{L}=290$ $\mathrm{nm}$. The calculated reflectance spectrum of the simulated structure presents a peak in $1450 \mathrm{~nm}$ for an air medium, as it is depicted in Fig. 3.34.

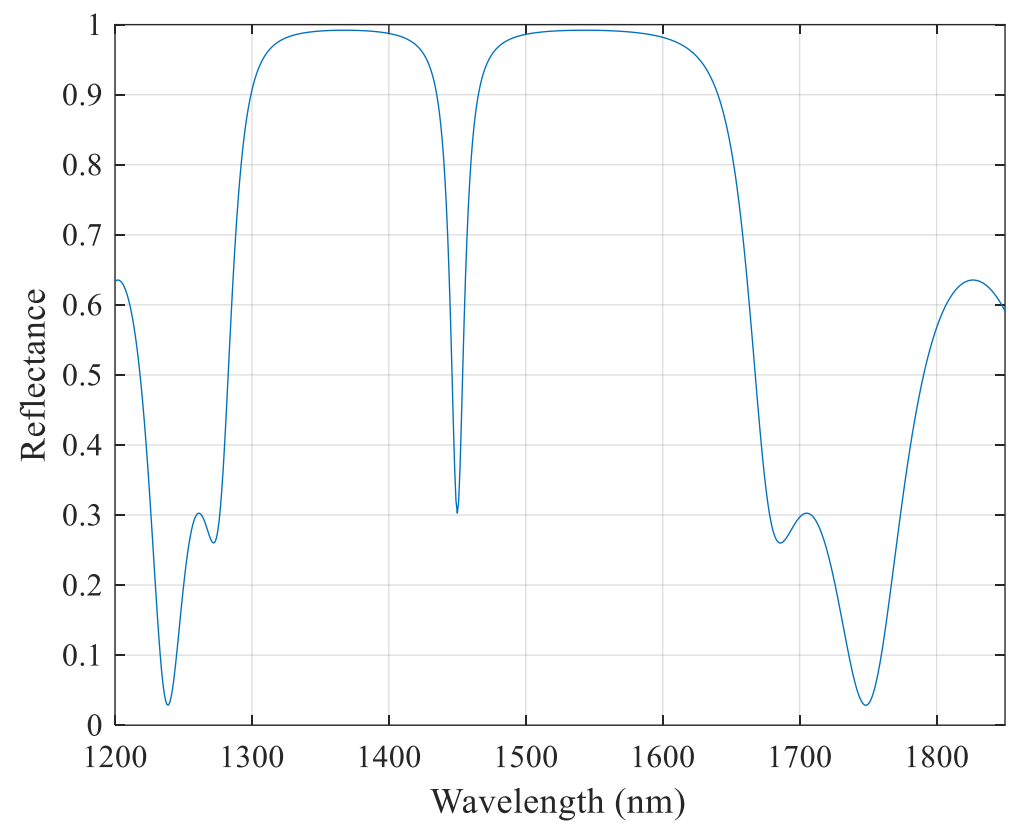

Fig. 3.34: Simulated reflectance spectrum of the designed PSMC.

The current densities and the etching times applied for the fabrication of the $\mathrm{n}_{\mathrm{H}}$ and $\mathrm{n}_{\mathrm{L}}$ layers were 16 and $55 \mathrm{~mA}, 10.3$ seconds and 6.9 seconds, respectively. Figure 3.35 shows a SEM cross-sectional image of the PSMC, where the light and dark grey layers are the $\mathrm{n}_{\mathrm{H}}$ and the $\mathrm{n}_{\mathrm{L}}$ layers, respectively. The average measured thicknesses of both layers are 202 and $279 \mathrm{~nm}$, respectively. The experimental RIs of the layers were calculated fitting the reflectance spectra with the TMM-based model. The obtained RIs were $\mathrm{n}_{\mathrm{H}}=1.75$ and $\mathrm{n}_{\mathrm{L}}=1.26$, corresponding to porosities of $65 \%$ and $83 \%$, respectively.

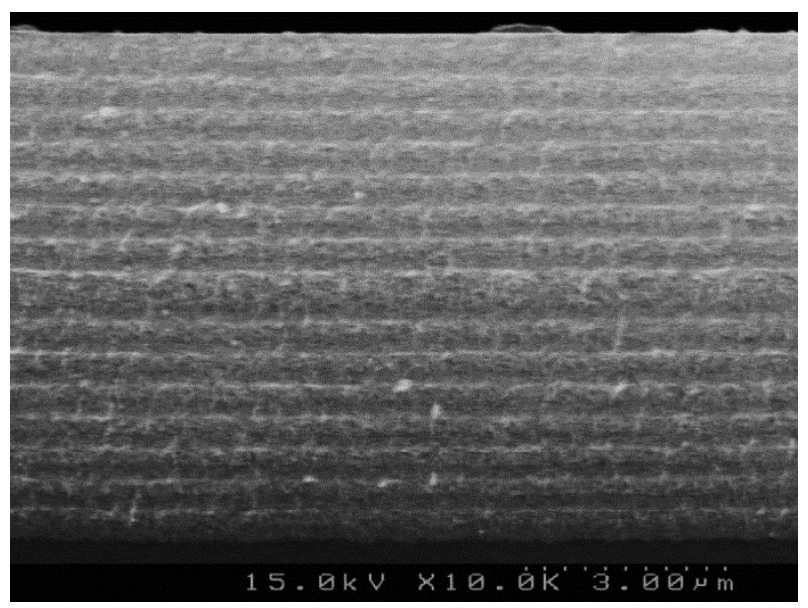

Fig. 3.35: SEM cross-sectional image of the fabricated PSMC. 
After the fabrication process, the PS sample was placed in the fluidic cell and its reflectivity spectrum was characterized in a DIW flow. In Fig. 3.36 the reflectivity spectrum is shown. As it is can be observed, the resonance peak was located at $1561 \mathrm{~nm}$.

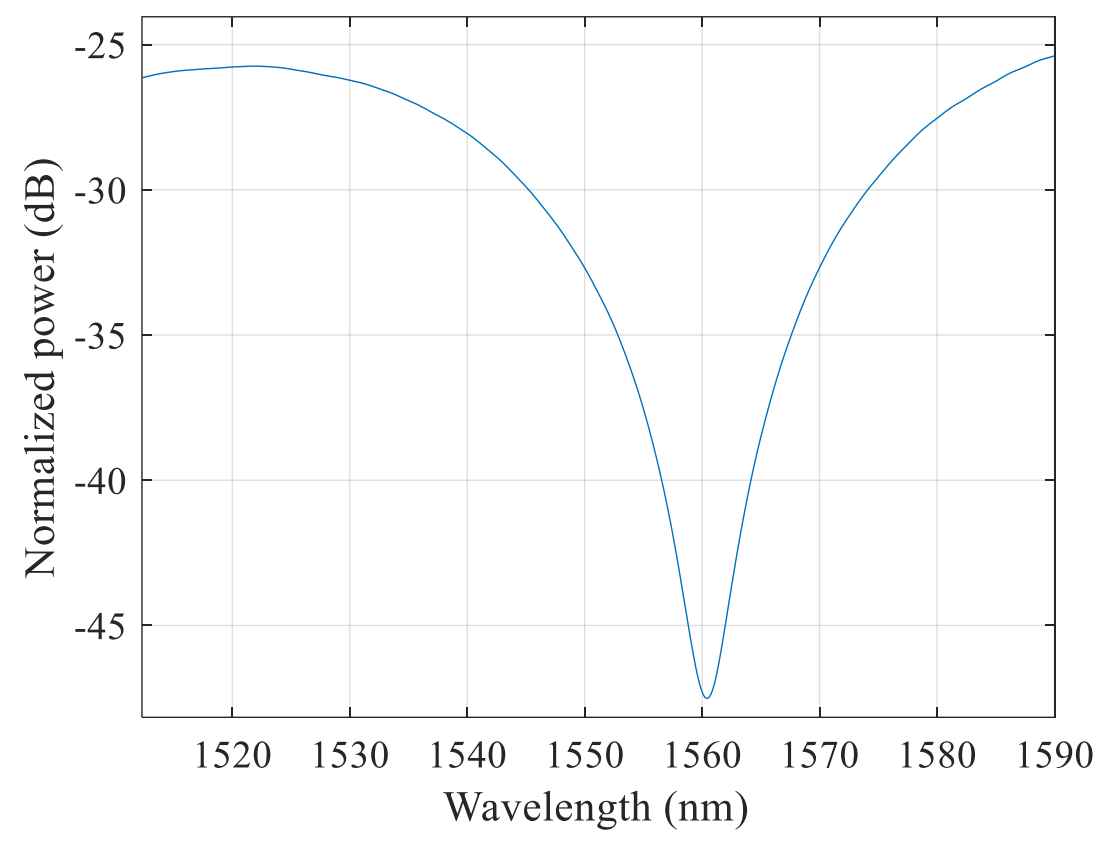

Fig. 3.36: Reflectance spectrum of the PSMC.

Several RI sensing experiments were performed in order to determine the sensitivity and the detection limit of the PSMC-based sensing system. The experimental sensing procedure consisted of flowing several EtOH dilutions in DIW over the PSMC sensor, and monitoring in real-time the evolution of the PSMC resonance peak when the RI was changed. The EtOH concentrations in DIW in these dilutions were $1 \%, 0.1 \%, 0.01 \%$ and $0.001 \%$, which means a relative RI change of $6.6 \times 10^{-4}, 6.6 \times 10^{-5}, 6.6 \times 10^{-6}$ and $6.6 \times 10^{-7} \mathrm{RIU}$, respectively. The temporal evolution of the resonance peak position for the mentioned EtOH concentrations is shown in Fig. 3.37. The RI changes and the relative shifts for each EtOH concentration are represented in Table 3.1. A noise in the range of only $0.8 \mathrm{pm}$ was measured in the experiments, which was further reduced by using an FFT algorithm in order to eliminate the high frequency components not related with the sensing, leading to a reduction of the noise by a factor $4 \times$. Because of this process, it was possible to clearly appreciate the spectral shifts that occurred at the lowest EtOH concentration. 

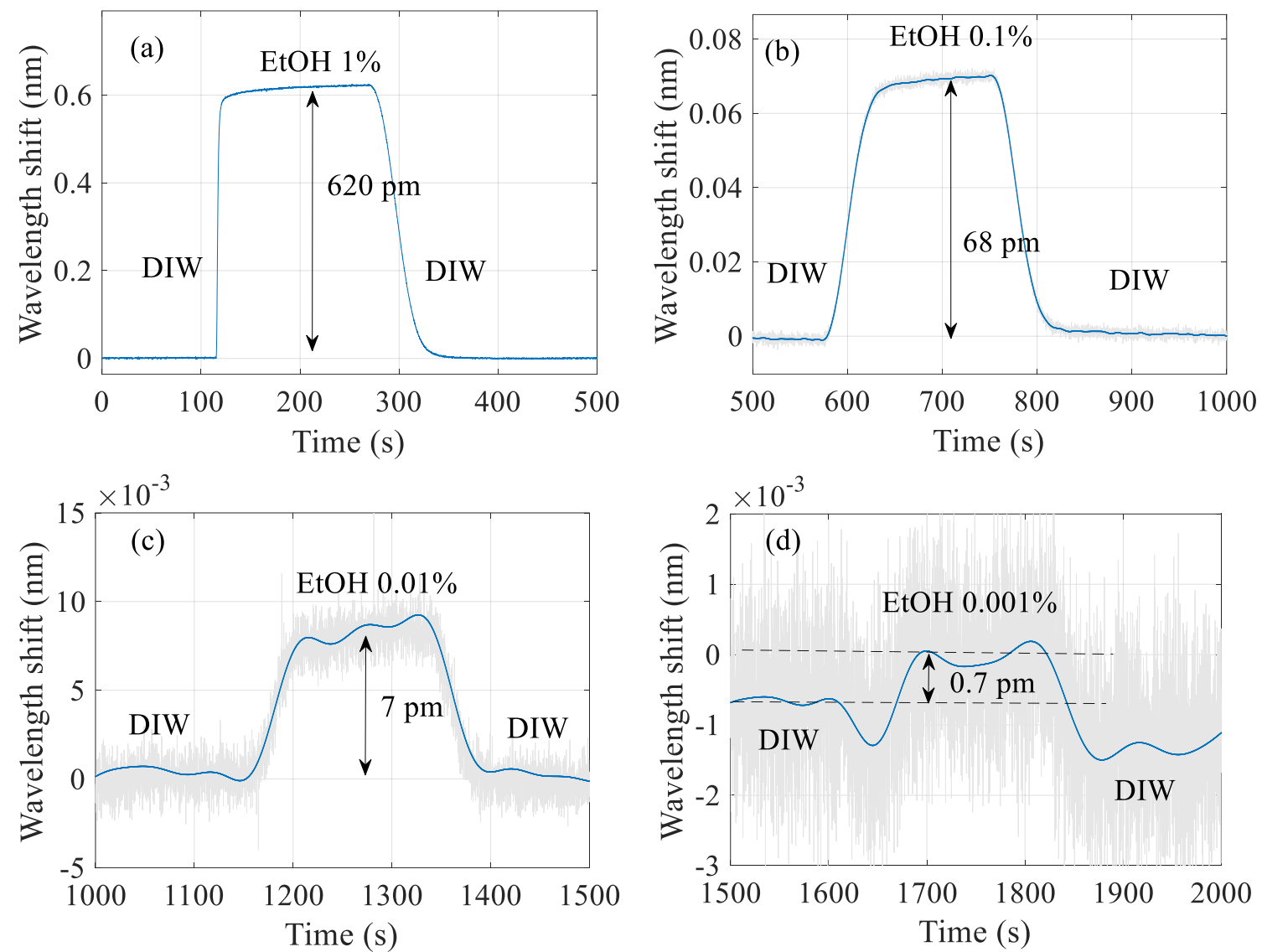

Fig. 3.37: Resonance peak wavelength shift for the EtOH concentrations (a) 1\%, (b) $0.1 \%$, (c) $0.01 \%$ and (d) $0.001 \%$. The raw data is shown in light grey, while the evolution after a noise filtering process is shown in blue.

\begin{tabular}{cccc}
\hline [EtOH] $(\%)$ & $\Delta$ RI (RIU) & $\Delta \lambda(\mathbf{p m})$ & {$[\mathbf{E t O H ]}(\boldsymbol{\%})$} \\
\hline 1 & $6.6 \times 10^{-4}$ & 620 & 1 \\
0.1 & $6.6 \times 10^{-5}$ & 68 & 0.1 \\
0.01 & $6.6 \times 10^{-6}$ & 7 & 0.01 \\
0.001 & $6.6 \times 10^{-7}$ & 0.7 & 0.001 \\
\hline
\end{tabular}

Table 3.1: Values of the refractive index changes of the several EtOH concentrations and of the relative PSMC resonance peak wavelength shift. 


\subsubsection{Results analysis}

Figure 3.38 shows the relationship between the PSMC resonance peak wavelength shifts and the RI variation for each EtOH concentration in logarithmic scale as well as the sensitivity curve. As it is shown in this graph, the PSMC presents a very linear behaviour for the measurement of RI variations over a very wide range of concentrations, allowing to measure values even in the $10^{-7}$ RIU range, as experimentally demonstrated. The PSMC exhibited a sensitivity around $1000 \mathrm{~nm} / \mathrm{RIU}$.

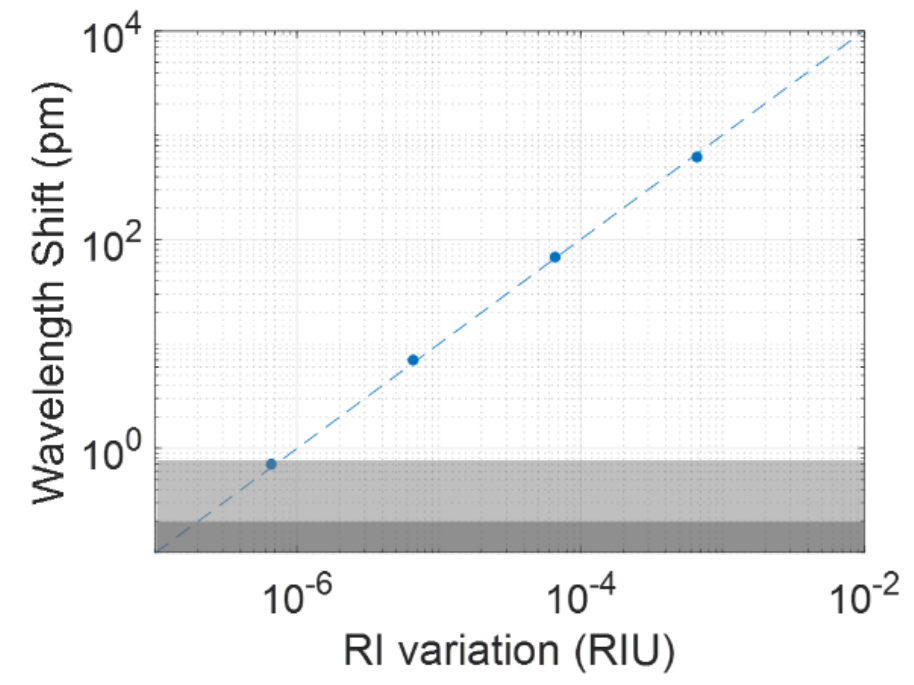

Fig. 3.38: Relationship between the PSMC resonance peak wavelength shift and the RI change in logarithmic scale. The light grey region represents the noise level for the experimental raw data, whereas the dark grey region represents the noise level after the filtering process.

Table 3.2 summarizes some of the main results reported in the field of the development of PS-based sensing structures, where details about the experimental conditions are also indicated. Regarding the sensitivity to RI variations, our result represents an improvement by a factor of at least $\sim 2 \times$ compared to the best results previously reported for the optimization of the porosity of the structure. Regarding the experimental conditions, only some of the works performed a continuous monitoring of the PS sensing structure while flowing the target substance over it. All of them made use of a spectrometer to acquire the spectrum, leading to a reported acquisition period of several seconds, a time at least one order of magnitude longer than that reported in this work. Additionally, typical spectral resolutions of those spectrometers are in the range of several hundreds of pm (compared to $4 \mathrm{pm}$ for the optical interrogator used in this work), which also limits the minimum spectral shift that can be accurately measured. 


\begin{tabular}{ccccccc}
\hline $\begin{array}{c}\text { PS } \\
\text { Structure }\end{array}$ & $\begin{array}{c}\text { Target } \\
\text { Substance }\end{array}$ & $\begin{array}{c}\text { Interrogation } \\
\text { Equipment }\end{array}$ & $\begin{array}{c}\text { Sensitivity } \\
(\mathbf{n m} / \mathbf{R I U})\end{array}$ & $\begin{array}{c}\text { Continuous } \\
\text { Flow }\end{array}$ & $\begin{array}{c}\text { Acquisition } \\
\text { Period }\end{array}$ & Reference \\
\hline MC & Sucrose & Spectrometer & $\mathrm{n} / \mathrm{a}$ & $\checkmark$ & $8 \mathrm{~s}$ & {$[40]$} \\
FP & Hydrogen & Spectrometer & $\mathrm{n} / \mathrm{a}$ & $\checkmark$ & $8 \mathrm{~s}$ & {$[82]$} \\
MC & Solvents & Spectrometer & 425 & $\mathrm{n} / \mathrm{a}$ & $\mathrm{n} / \mathrm{a}$ & {$[69]$} \\
BR & Gas & Spectrometer & $470 \pm 40$ & $\mathrm{n} / \mathrm{a}$ & $\mathrm{n} / \mathrm{a}$ & {$[70]$} \\
MC & Solvents & Spectrometer & $\sim 550$ & $\mathrm{n} / \mathrm{a}$ & $\mathrm{n} / \mathrm{a}$ & {$[71]$} \\
DL & Proteases & Spectrometer & $\mathrm{n} / \mathrm{a}$ & $\checkmark$ & $2 \mathrm{~s}$ & {$[83]$} \\
MC & Ethanol & Spectrometer & $\sim 350$ & $\checkmark$ & $4 \mathrm{~s}$ & {$[72]$} \\
MC & Solvents & Spectrometer & 200 & $\checkmark$ & $\mathrm{n} / \mathrm{a}$ & {$[84]$} \\
MC & Streptavidin & Spectrometer & $\mathrm{n} / \mathrm{a}$ & $\checkmark$ & $20 \mathrm{~s}$ & {$[74]$} \\
MC & Ethanol & Optical interrogator & $\sim 1000$ & $\checkmark$ & $0.1 \mathrm{~s}$ & This work \\
\hline
\end{tabular}

Table 3.2. Review of experimental results reported using PS-based sensing structures. Several configurations of the PS sensing structures are considered (SL: single layer, FP: Fabry-Perot, DL: double layer, BR: Bragg reflector, MC: microcavity).

\subsubsection{Conclusions}

In this chapter, we have presented the study of the use of PS 1D periodic structures for sensing purposes. In the first part of the chapter, the use of a Bragg reflector as photonic sensing structure and the power sensing technique have been described. The results indicate a high sensitivity, what makes possible the detection of low RI variations. However, the measured power evolution was not linear, owing to the power dependence on the slope of the interval selected to be monitored. However, measurements based on the tracking of the wavelength shift showed the same sensitivity for all the RI variations. In the second part of this chapter, the use of a porous silicon microcavity as an optical sensor for the detection of RI variations was described. Extremely low RI variations were detected.

Thanks to the experimental opto-fluidic setup, the detection occurred in real-time. The experimental results indicate that the PSMC presented a sensitivity as high as $1000 \mathrm{~nm} / \mathrm{RIU}$ and a limit of detection in the $10^{-7}$ RIU range. The achievement of such high sensitivity confirmed what stated in the chapter 1: the fact that the sensing occurs directly inside the photonic structure leads to a higher light-matter interaction, and, thus, to higher sensitives. This is the contrary to what occurs for traditional solid core photonic structures based on the sensing with evanescence wave, where only a small portion of the optical field interacts with the target substance. Considering this phenomenon, the porosities of the layers were optimized in order to achieve an as-high-as-possible sensitivity, while maintaining the robustness of the structure. Furthermore, such experimental results were obtained in real-time due to the opto-fluidic setup developed to realize the experiments, which allowed us to monitor in-continuum the PSMC spectrum evolution with a very high spectral resolution. 


\section{Chapter 4}

\section{Temperature sensing using porous silicon fiber-optic sensing probe}

\subsection{Introduction}

In chapter 3, we have validated the use of PS structures for refractive index sensing by demonstrating the detection of extremely low refractive index variations. The following step was to demonstrate the use of this PS structure even for the sensing of physical parameters. In fact, even though a high refractive index sensitivity is a basic requirement for a photonic sensor, in sensing applications, also the monitoring of environmental parameters, including temperature, humidity and pressure, has to be considered.

Among these parameters, in this $\mathrm{PhD}$ Thesis, we focused the attention on the environmental temperature. In fact, the variation of temperature is crucial in several fields, especially research laboratories, food industries, environmental monitoring, monitoring of biochemical reactions or military and aerospace uses [85-86]. In this chapter, we present a novel temperature sensing configuration arisen from the combination of the well-known fiber-optic technology and a PS structure as optical sensing element. As PS optical sensor we selected the PSMC. 
An overview of several fiber-optic sensing configurations is provided in section 4.2. In section 4.3, we expose the process to develop the porous silicon sensing probe used as sensing element. The experimental setup developed to carry out the temperature sensing measurements is described in section 4.4. The experimental results are reported in section 4.5 and the analysis of these results is provided in section 4.6. Finally, in section 4.7, the conclusions are commented.

\subsection{Fiber-optic sensing overview}

Fiber-optic sensors (FOSs) have been widely studied and exploited becoming one of the principal optical sensing technologies due to the inexpensive cost and physical properties of the optical fiber [87-90]. Nowadays, FOSs are applied in several sensing applications such as for the measurement of refractive index, strain, temperature, pressure, vibration or bending $[88,91,92]$. This sensing versatility is due to the possibility of developing different types of FOSs where the selected parameter affects the intensity, frequency, polarization or phase of the light travelling through the fiber [89]. Furthermore, they offer several advantages including high sensitivity, immunity to electromagnetic interferences, small dimensions, cost effectiveness and the ability to operate in a wide range of environments [90].

Nowadays, various FOSs configurations have been reported in the literatures. Among them, fiber Bragg gratings (FBGs) [87, 89, 93], long period gratings (LPGs) [94] and Fabry-Perot (FP) cavities [95] are probably the configurations mostly used for sensing purposes. FBG are obtained by inscribing a periodic modulation of the refractive index in the core of the optical fiber itself, as shown in Fig. 4.1 (a). This modulation provokes the appearance of a range of reflected wavelengths that depends on the period, the refractive index of the structure, and the refractive index on the surroundings of the structure. LPG are similar to FBGs. In this configuration, the modulation pattern is in the order of a fraction of millimetres, as shown in Fig. 4.1 (b). FP cavities can be created by different methods including tapering the fiber or inserting a hollow fiber between two standard fibers, as shown in Fig. 4.1 (c). The characteristic spectrum of these FPbased sensors can be monitored in order to relate the measured spectral shift with the refractive index variation due to the molecular bindings. 

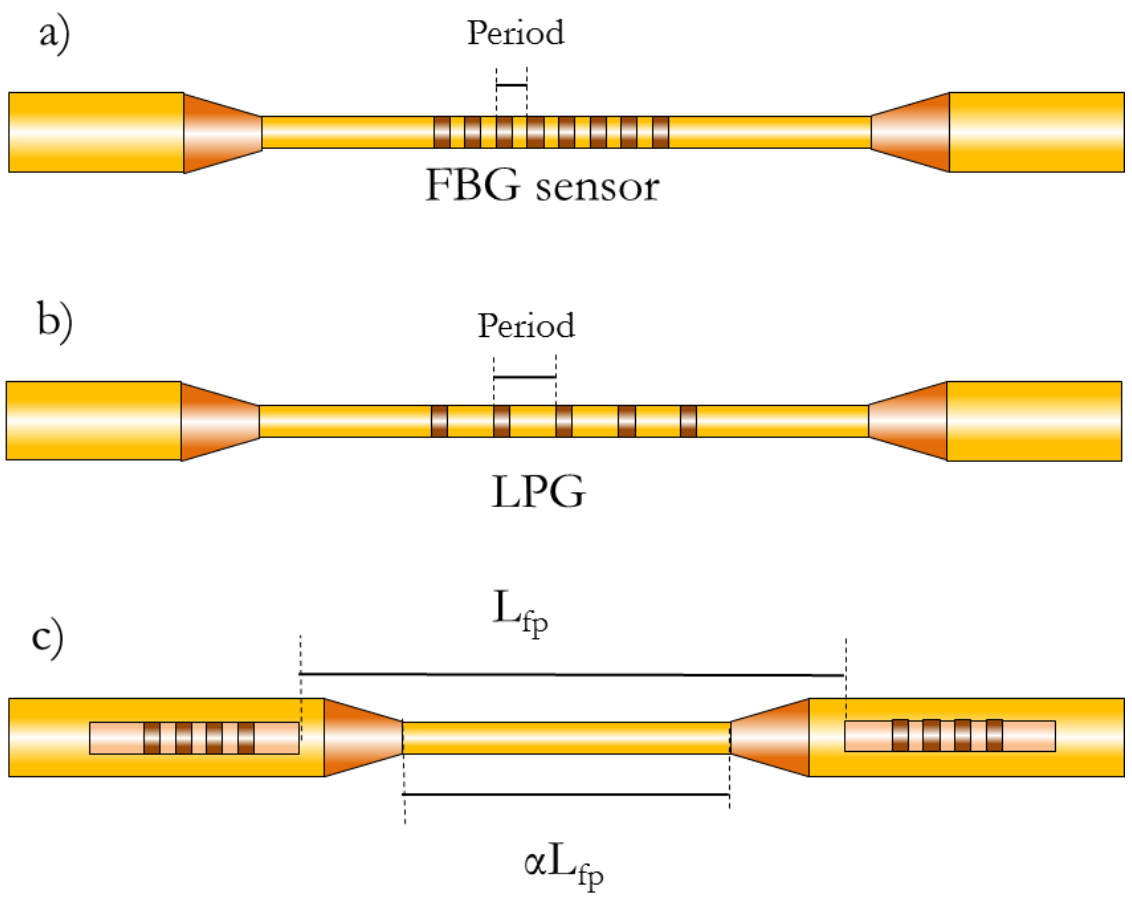

Fig. 4.1: Schematic illustration of a) FBG inscribed in a fiber, b) LPG structure, c) Fabry-Perot cavity based on two FBGs

It has to be pointed out that FOSs are finding a great success in temperature sensing [88-90, 93]. Presently the majority FOS configurations are based on fiber interferometers [96] and mainly on FBGs [87, 89, 93, 97]. Several works regarding temperature sensing using FBGs have been reported, where such structures exhibited a temperature sensitivity in the range of 10 $\mathrm{pm} /{ }^{\circ} \mathrm{C}[88,98]$.

Despite most temperature FOSs make use of the fiber itself as sensing element, this fact entails two main limitations. The first one consists in the relatively complex fabrication of the sensor, since the optical sensing structure has to be directly fabricated on the fiber itself. These sensors are rather expensive to produce and complicated to implement on-site [99]. The second one is related with the thermal properties of the fiber-based sensors, namely, the thermo-optic coefficient (TOC), i.e., the variation of the refractive index as a function of the temperature, and the thermal diffusivity, i.e., the temperature transfer rate of the material. Since the optical fiber is made of silica, it is characterized by relatively low TOC and thermal diffusivity. A low TOC determines a low sensitivity towards temperature changes, whereas a low thermal diffusivity implies a larger time needed by the structure to reach the required temperature. In conclusion, since the temperature sensitivity is determined mainly by the material of the FBGs, these structures exhibit low temperature sensitivity values.

Several techniques have been used to further enhance the sensitivity and also to reduce the response time. Among them, a widely employed technique is using a metal package for the 
FBGs, leading to a sensitivity in the range of $27 \mathrm{pm} /{ }^{\circ} \mathrm{C}$ and a real-time response [100]. However, this process increases the complexity and the cost for the development of such a temperature sensor. An alternative to FBGs are LPGs. These sensors can reach a sensitivity in the range of $100 \mathrm{pm} /{ }^{\circ} \mathrm{C}$, but their high bending sensitivity may give rise to variations of the spectral response not related with the temperature variations [101, 102]. Furthermore, also this type of configuration presents the limitation of a complex fabrication.

The FOS probe configuration presented in this chapter is an effective solution to these limitations. The configuration was based on the combination of an optical fiber and an additional optical sensing element placed on the tip of the optical fiber itself. PSMC was chosen as additional element, due to its high temperature sensitivity, cheapness and easy and quick fabrication. In this configuration, the sensing element was not the optical fiber component anymore, but we transferred such a role to the PSMC. By doing this, we were able to eliminate the typical issues of the FOS configurations previously introduced, regarding the complexity of the fabrication and the limitation of the temperature sensitivity.

\subsection{PSMC sensing probe fabrication}

In this study, the PSMC structure design used as sensing element was similar to that used for RI sensing in chapter 3. The only difference consisted in the reflectance spectrum, which, in this case, presented the resonance peak at around 1550 in air environment. So, the model based on the TMM was used to determine the structure parameters $n_{H}, n_{L}, d_{H}$, and $d_{L}$ in order to obtain a resonant peak located at $1550 \mathrm{~nm}$. The refractive indices and the corresponding thicknesses obtained in the design process were $\mathrm{n}_{\mathrm{H}}=1.70, \mathrm{n}_{\mathrm{L}}=1.40, \mathrm{~d}_{\mathrm{H}}=228 \mathrm{~nm}$ and $\mathrm{d}_{\mathrm{L}}=278 \mathrm{~nm}$. The theoretical porosities for the $\mathrm{n}_{H}$ and $\mathrm{n}_{\mathrm{L}}$ layers were $65 \%$ and $77 \%$, respectively.

In order to create the $n_{H}$ and $n_{L}$ layers, current densities of $16 \mathrm{~mA} / \mathrm{cm}^{2}$ and $45 \mathrm{~mA} / \mathrm{cm}^{2}$ and etching times of 10.3 seconds and 6.9 seconds were selected. Figure 4.2 shows a SEM crosssectional image of the PSMC, where the light and dark grey layers are the $\mathrm{n}_{\mathrm{H}}$ and the $\mathrm{n}_{\mathrm{L}}$ layers, respectively. The thicknesses measured with SEM of the $n_{H}$ and $n_{L}$ the layers were $215 \mathrm{~nm}$ and $265 \mathrm{~nm}$, respectively. The estimated refractive index values are $n_{H}=1.92$ and $n_{L}=1.42$, whereas the relative porosities were $57 \%$ and $75 \%$, respectively. 


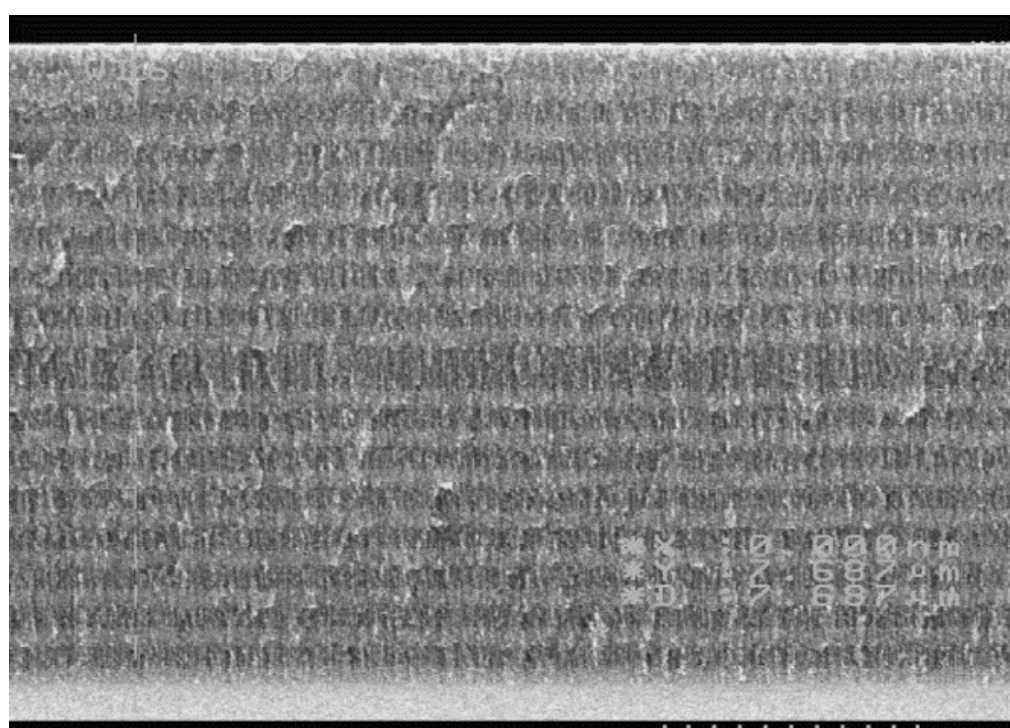

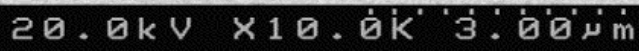

Fig. 4.2: (a) SEM cross-sectional image of the fabricated PSMC sample.

The preparation of the PSMC sensing probe is schematically shown in Fig. 4.3. The PSMC sample created by electrochemical etching was diced into square pieces of size $1 \mathrm{~mm}^{2}$ by using an automatic dicing saw. This small size will allow us to measure the temperature variations at a very specific point. The dicing process required around 6 minutes. In this way, considering the electrochemical etching and the dicing processes, around 90 PSMC sensors were obtained in 10 minutes. One of these square pieces was selected and attached to the tip of an optical fiber connector with the porous side facing it. To this aim, epoxy glue was used since it is transparent at $1550 \mathrm{~nm}$.
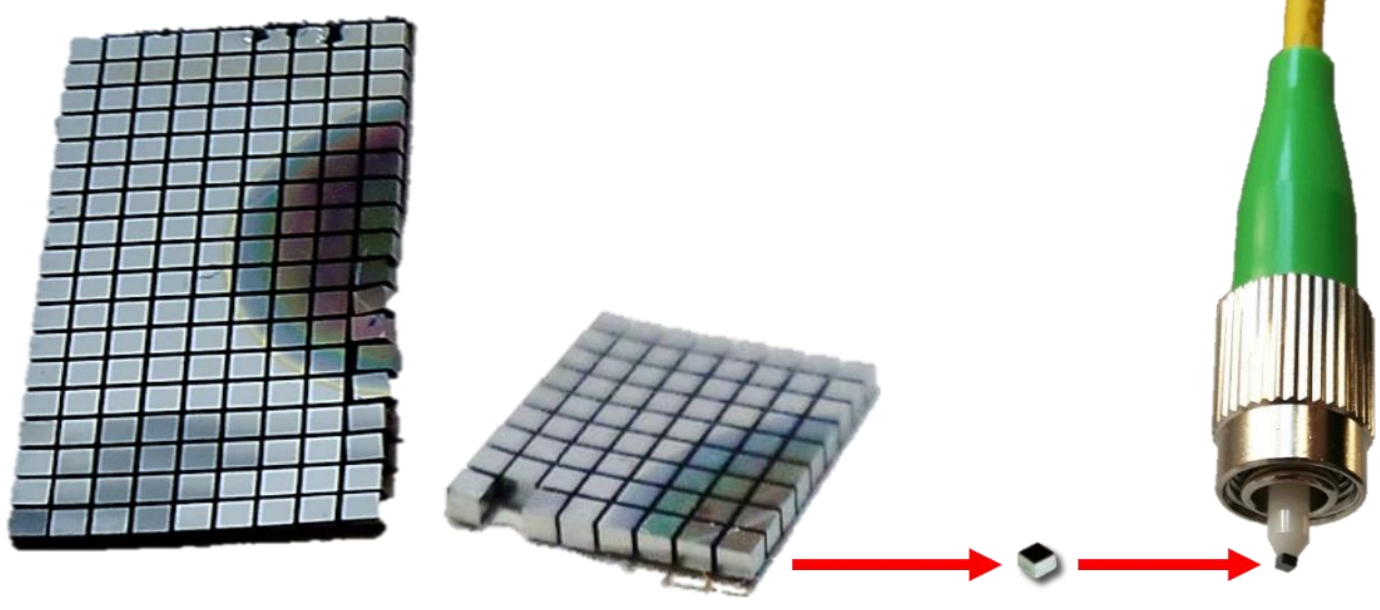

Fig. 4.3: Illustration of the development of the PSMC sensing probe. 


\subsection{Experimental opto-thermal setup}

In order to characterize and monitor in real-time the PSMC spectrum, the optical part of the experimental setup developed for the RI sensing experiments, described in chapter 3, was used as shown in Fig. 4.4. In this configuration, the PSMC sensing probe was directly connected to optical interrogator.

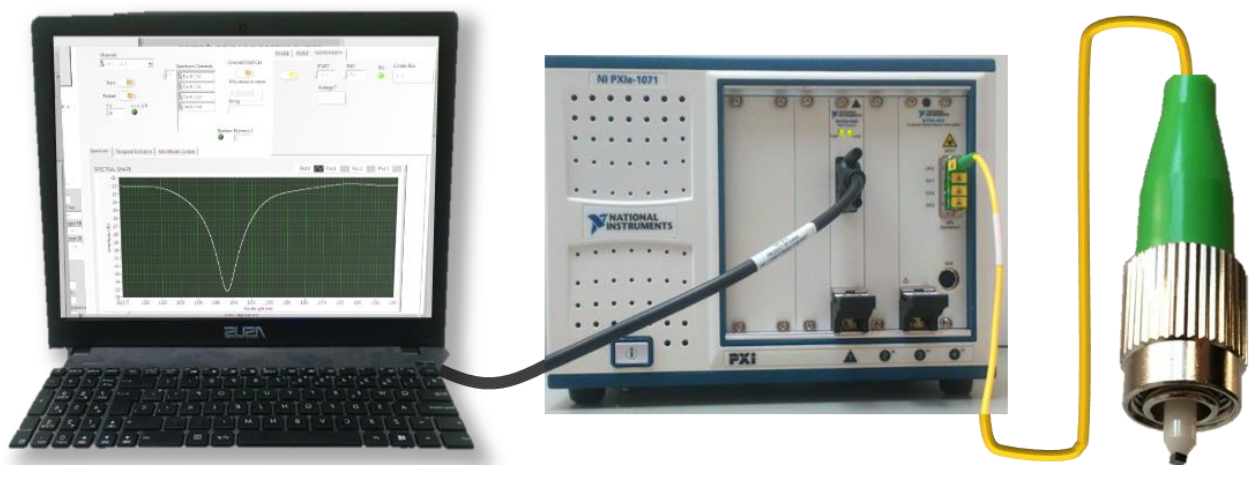

Fig. 4.4: Schematic illustration of the optical setup configuration used to monitor the PSMC spectrum.

In order to control the temperature of the system, a thermal setup was developed, as shown in Fig. 4.5. A Peltier cell was used as heater (or cooler). To control the Peltier cell, a LDC3744C Precision Laser Diode Controller was used as temperature controller (TC). Finally, a thermocouple was used as temperature sensor to close the loop of the instrument internal controller. Using thermal paste, the Peltier cell and the thermocouple were attached and thermally connected to a heatsink. The Peltier cell heated or cooled the heatsink until the thermocouple reached the input temperature. In order to know the temperature being perceived by the PSMC sensing probe, a metallic stick temperature sensor was used as reference sensor.

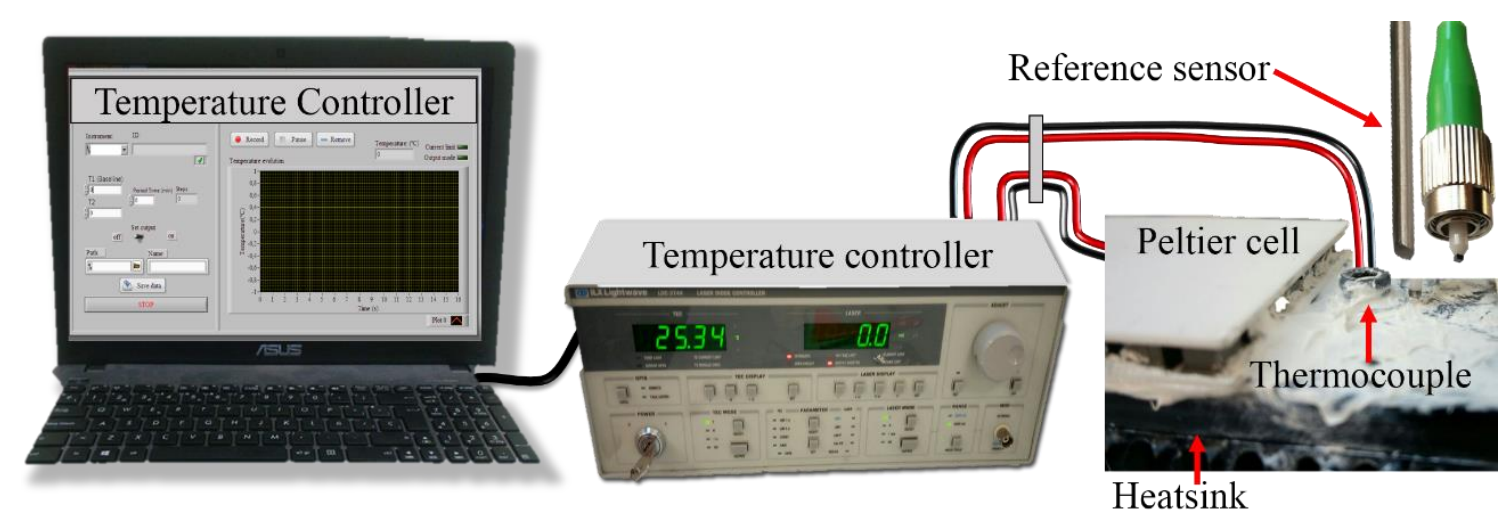

Fig. 4.5: Illustration of the thermal setup. 
The two setups were combined giving rise to an opto-thermal setup capable to change the temperature of the system and simultaneously monitor the spectrum evolution. Figure 4.6 shows a schematic illustration of the whole experimental setup.

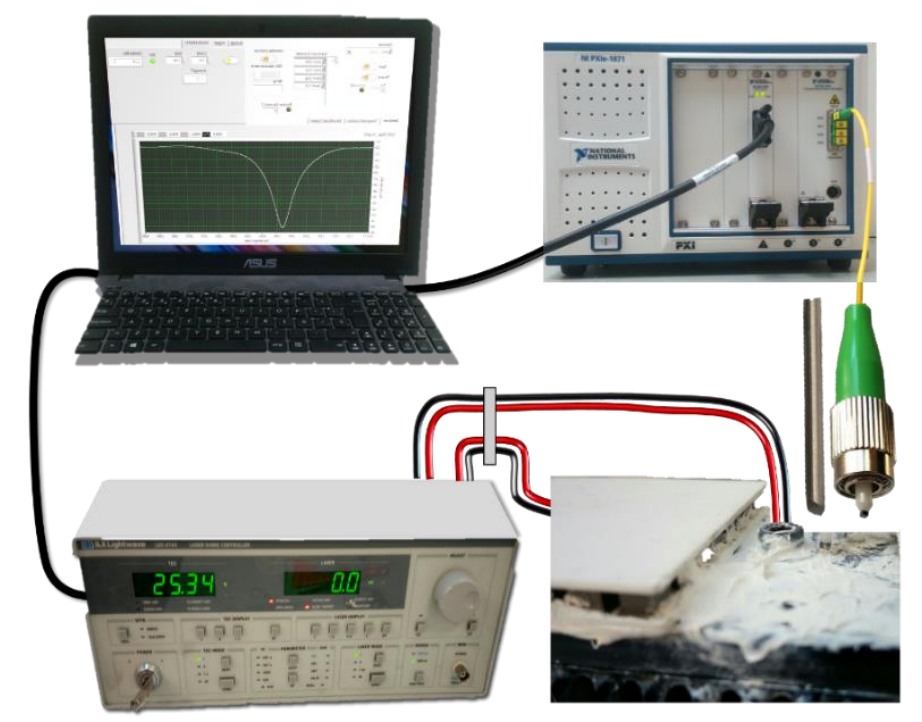

Fig. 4.6: Illustration of the opto-thermal setup used in the experiments.

First, experiments in water environment were carried in order to study the performance of the sensor and, then, experiments in air environment were carried out in order to compare the results with those obtained in water environment and study how the temperature increases affect to the noise. To this aim, two different configurations were considered to monitor the temperature in water and in air environment, schematically illustrated in Fig. 4.7. In the sensing-in-water configuration, shown in Fig. 4.7 (a), the PSMC sensing probe was immersed in a water-filled beaker. This beaker was placed on the heatsink where it was thermally connected with thermal paste. In the sensing-in-air configuration, shown in Fig. 4.7 (b) the sensing probe was placed above the heatsink, close to the thermocouple, at a distance of $2 \mathrm{~mm}$.

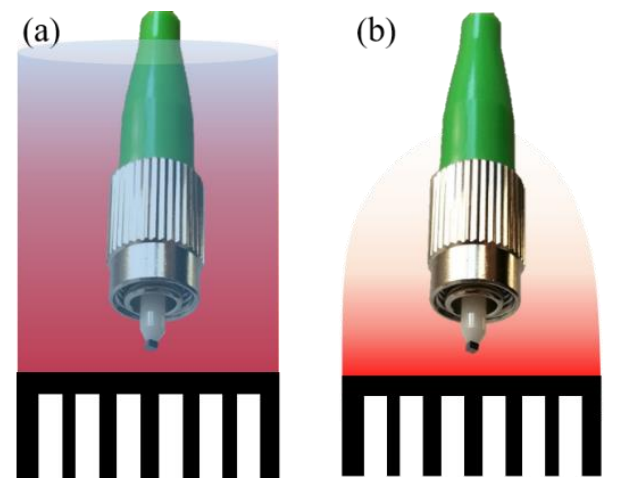

Fig. 4.7: Schematic representation of the (a) sensing-in-water and (b) sensing-in-air configurations. 


\subsection{Experimental temperature sensing results}

The reflectance spectrum provided by the fabricated PSMC structure is shown in Fig. 4.8. As it can be observed, the spectrum presents a resonance peak in $1532 \mathrm{~nm}$, close to the position expected from the design stage. Such experimental results are in a good agreement with those calculated in the design stage. The positive outcome of the PSMC spectrum was due to the correct configuration of the calculated etching process parameters. The temperature sensing experiment were performed by monitoring the position of this resonance peak.

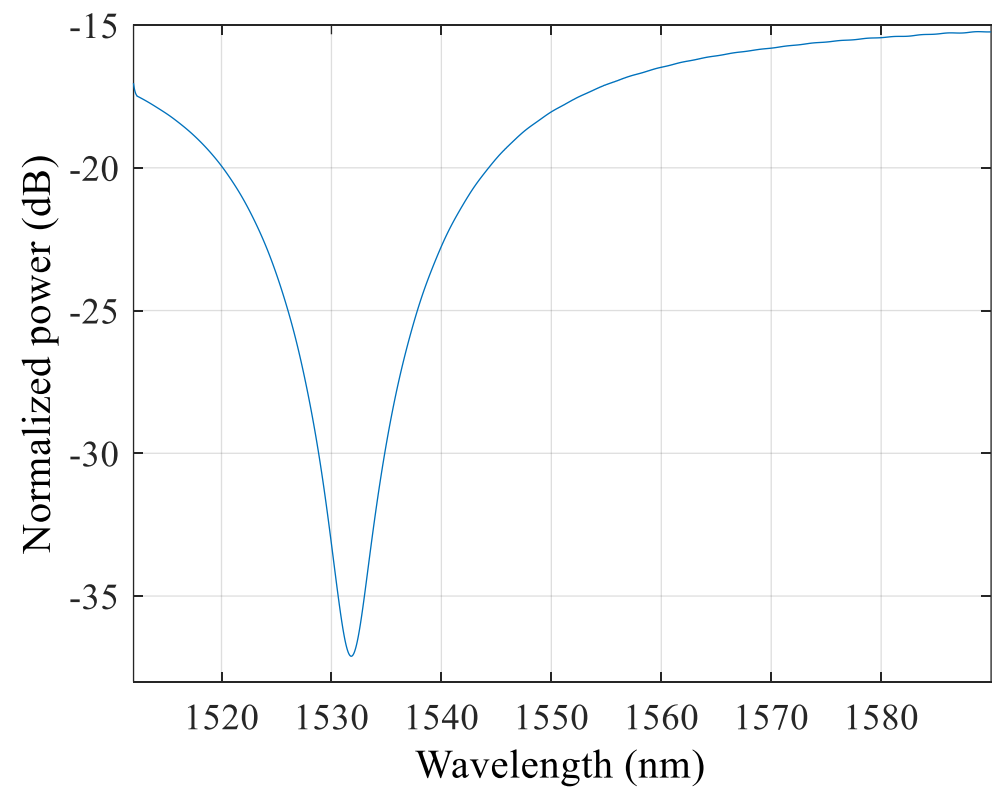

Fig. 4.8: Reflectance spectrum of the PSMC used as temperature sensing element.

As explained in chapter 3 , the resonance peak position depends on the refractive index and the thickness of the layers. However, these two parameters depend on the temperature since the refractive index will change due to the thermo-optic effect, whereas the layer thickness will change due to the thermal expansion. This means that temperature variations cause variations of the refractive index, of the thickness and of the pore diameter of the PS structure. The variations of these parameters contribute to the shift of the resonance peak. In summary, temperature variations can be determined by monitoring the resonance peak shift caused by the variation of such parameters. In Fig. 4.9, an illustration of the temperature sensing mechanism is shown. 


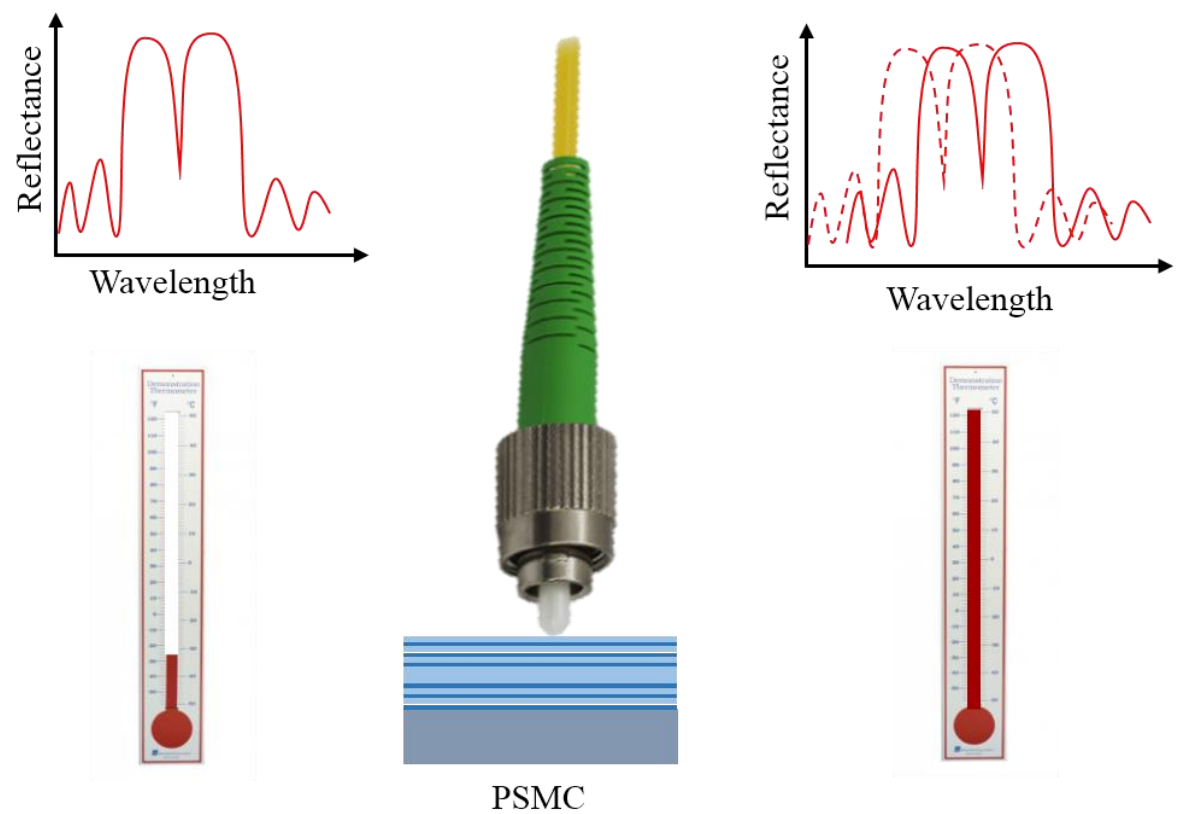

Fig. 4.9: Schematic illustration of the temperature sensing mechanism.

\subsubsection{Thermal setup performance analysis}

Before carrying out the temperature sensing experiments with the sensing probe, the performance of the thermal setup was studied. As shown in Fig. 4.10, the TC input temperature was increased from $24{ }^{\circ} \mathrm{C}$ to $25^{\circ} \mathrm{C}$ and the temperature perceived by the thermocouple was monitored in real-time and in-continuum. The average observed noise was $6 \cdot 10^{-3}{ }^{\circ} \mathrm{C}$. Such noise was produced by the constant correction of the TC to keep the temperature at the input value. The time needed by the thermocouple to reach the increment of $1{ }^{\circ} \mathrm{C}$ was around 30 seconds. This value represents the time needed by the Peltier cell to heat the heatsink and thus the thermocouple.
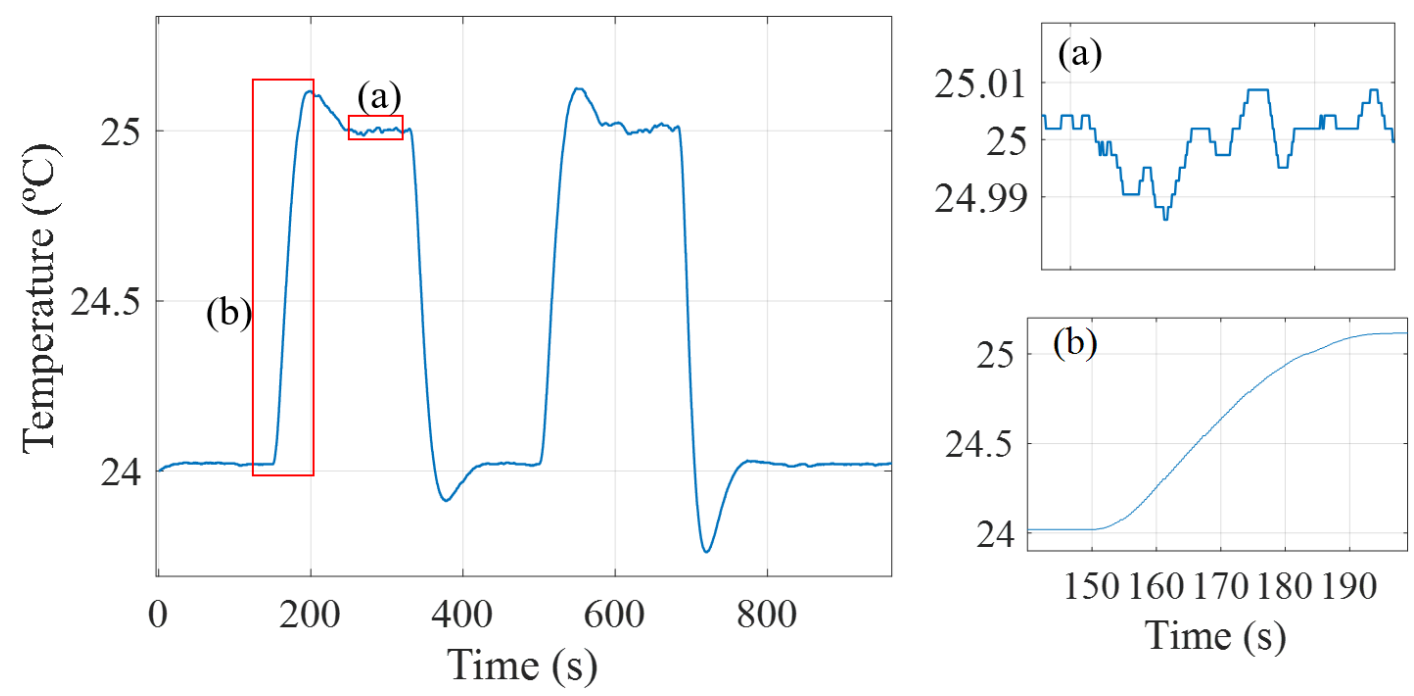

Fig. 4.10: Time evolution of the temperature perceived by the thermocouple. The inset (a) depicts the noise of the system, whereas the inset (b) depicts its response time. 


\subsubsection{Water environment experiments}

Experiments in water environment were carried out first in order to characterize the sensitivity and the resolution of our PSMC sensing probe. The experiments were performed by constantly controlling the heatsink temperature and its variation in a degree-by-degree basis. At the same time, the evolution of the temperature perceived by the reference sensor was monitored. The evolution of the PSMC resonance peak was monitored in real-time.

The temperature changes applied to the heatsink were from $24^{\circ} \mathrm{C}$ to $25^{\circ} \mathrm{C}$, from $26^{\circ} \mathrm{C}$ to $27^{\circ} \mathrm{C}$, from $28^{\circ} \mathrm{C}$ to $29^{\circ} \mathrm{C}$ and from $30^{\circ} \mathrm{C}$ to $31^{\circ} \mathrm{C}$. As a result of each temperature change, a temperature variation of $0.2^{\circ} \mathrm{C}$ was measured by the reference sensor; so, that will be the temperature variation to be really measured by the PSMC sensing probe (i.e., not the $1^{\circ} \mathrm{C}$ variation applied to the heatsink). Figure 4.11 shows the PSMC resonance peak time evolution relative to the temperature changes previously indicated. A resonance peak shift of $22 \pm 1 \mathrm{pm}$ was observed in all the cases for the temperature change of $0.2{ }^{\circ} \mathrm{C}$ that was measured by the metallic reference sensor, thus leading to a sensitivity of $110 \pm 5 \mathrm{pm} /{ }^{\circ} \mathrm{C}$ of the PSMC sensing probe. The average measured experimental noise was $0.6 \mathrm{pm}$. The noise level was further reduced by around an order of magnitude by using a FFT filtering algorithm in order to eliminate the high frequency components not related with the sensing. Thanks to such noise reduction process, a noise value of $0.07 \mathrm{pm}$ was calculated, what leads to a temperature resolution of $6.3 \cdot 10^{-4}{ }^{\circ} \mathrm{C}$. Finally, the time required by the system to reach the plateau for each temperature change was around 90 seconds.
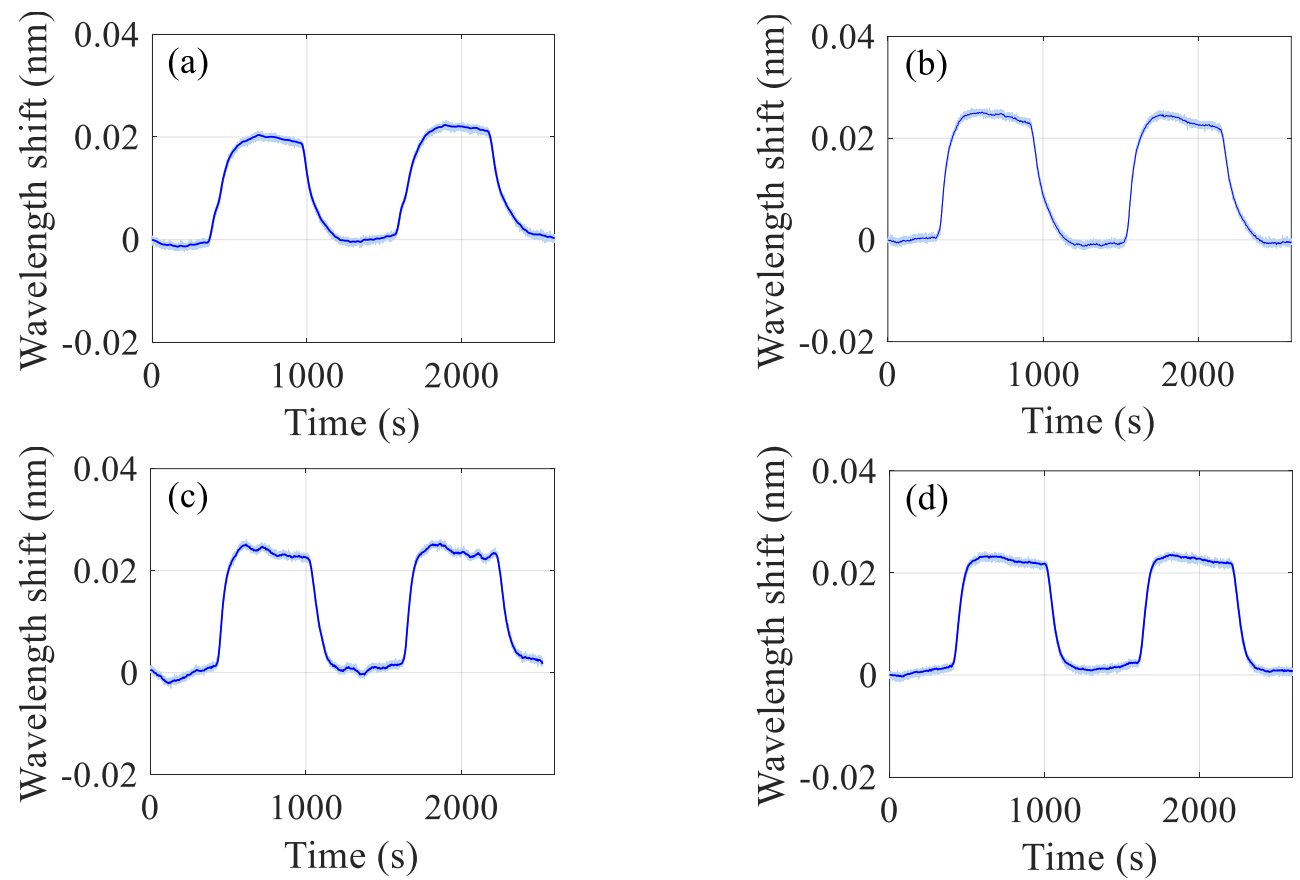

Fig. 4.11: PSMC peak time evolution relative to the heatsink temperature change (a) from $24^{\circ} \mathrm{C}$ to $25^{\circ} \mathrm{C}$, (b) from $26^{\circ} \mathrm{C}$ to $27^{\circ} \mathrm{C}$, (c) from $28^{\circ} \mathrm{C}$ to $29^{\circ} \mathrm{C}$ and (d) from $30^{\circ} \mathrm{C}$ to $31^{\circ} \mathrm{C}$. The experimental evolution is represented in light blue, whereas the evolution after the noise filtering process is represented in dark blue. 


\subsubsection{Air environment experiments}

Once the PSMC sensing probe performance was characterized, experiments in air environment were carried out. The temperature changes applied to the heatsink were from $23{ }^{\circ} \mathrm{C}$ to $24{ }^{\circ} \mathrm{C}$, from $24{ }^{\circ} \mathrm{C}$ to $25^{\circ} \mathrm{C}$, from $26^{\circ} \mathrm{C}$ to $27^{\circ} \mathrm{C}$ and from $28^{\circ} \mathrm{C}$ to $29^{\circ} \mathrm{C}$. Figure 4.12 shows the PSMC resonance peak time evolutions relative to these temperature changes.
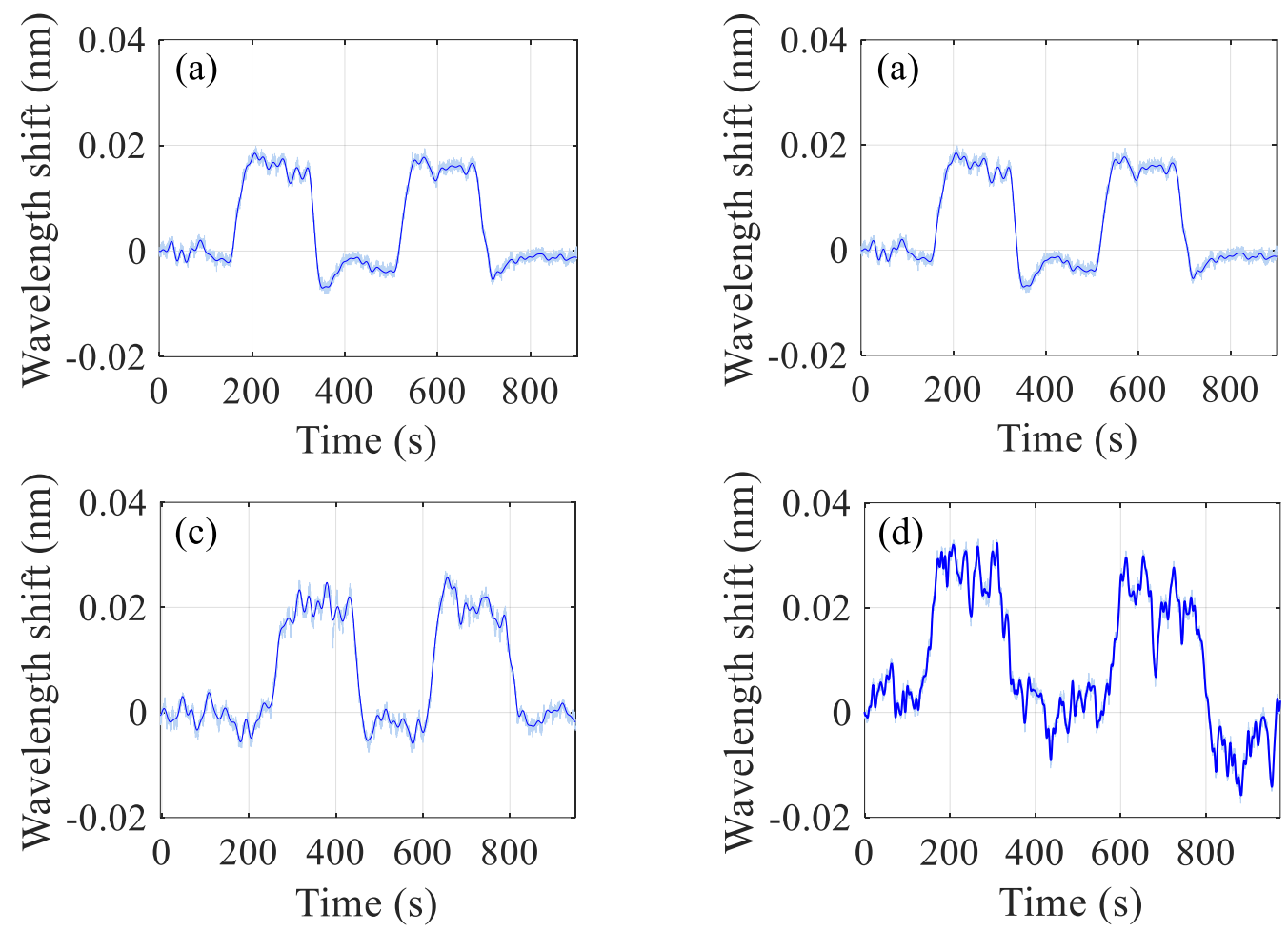

Fig. 4.12: PSMC peak time evolution relative to the heatsink temperature change (a) from $23^{\circ} \mathrm{C}$ to $24^{\circ} \mathrm{C}$, (b) from $24^{\circ} \mathrm{C}$ to $25^{\circ} \mathrm{C}$, (c) from $26^{\circ} \mathrm{C}$ to $27^{\circ} \mathrm{C}$ and (d) from $28^{\circ} \mathrm{C}$ to $29^{\circ} \mathrm{C}$. The experimental evolution is represented in light blue, while the evolution after a noise filtering process is represented in dark blue.

Analysing the evolutions, it is possible to observe that the average value of the noise was considerably higher than that observed in water environment experiments. Moreover, the noise increased for higher initial temperature values. The noise values of the experiments in air environment are reported in Table 4.1 and represented in Fig. 4.13, confirming what observed in Fig. 4.12. Finally, the average time needed to reach the plateau is around 30 seconds.

\begin{tabular}{cc}
\hline Temperature change $\left({ }^{\mathbf{O}} \mathbf{C}\right)$ & Noise (pm) \\
\hline $23-24$ & 1.1 \\
$24-25$ & 1.4 \\
$26-27$ & 2.1 \\
$28-29$ & 3.4 \\
\hline
\end{tabular}

Table 4.1: Values of the noise obtained in the experiments in air environment. 


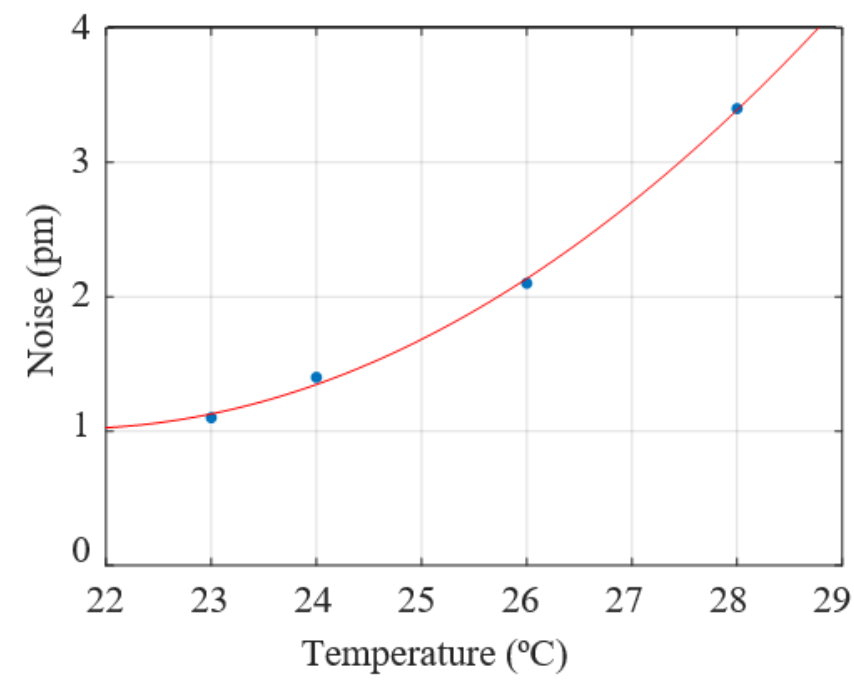

Fig. 4.13: Relationship between the measured noise and the initial heatsink temperature value used in the air environment experiments.

\subsection{Results analysis}

The results obtained from the characterization of the thermal system, the experiments in water and in air environment led us to understand clearly all the performance of our PSMC sensing probe. In Table 4.2, the experimental performance of the thermal setup and the PSMC sensing probe are reported.

\begin{tabular}{ccc}
\hline System & Noise & Response time \\
\hline Thermal setup & $6 \cdot 10^{-3}{ }^{\circ} \mathrm{C}$ & 30 seconds \\
PSMC in water & $0.6 \mathrm{pm}$ & 90 seconds \\
PSMC in air & $>1.1 \mathrm{pm}$ & $\sim 30$ seconds \\
\hline
\end{tabular}

Table 4.2: Values of the experimental performance of the thermal setup and obtained in the experiments in water and air environment.

\subsubsection{Noise analysis}

In water environment, a noise value of just $0.6 \mathrm{pm}$ was obtained. Considering that the sensitivity of our sensor was $110 \pm 5 \mathrm{pm} /{ }^{\circ} \mathrm{C}$, this noise corresponds to a temperature resolution of $6 \cdot 10^{-3}{ }^{\circ} \mathrm{C}$. This value is the same previously measured for the TC. This indicates that the measured resolution for the PSMC sensing probe is actually limited by the thermal setup performance and that it could be higher. Such result demonstrates that our PSMC temperature sensing probe is able to measure very small temperature variations within a very specific point. 
In air environment, a higher level of noise was observed. This was due to the perturbations produced by the air environment, which is a less stable system than water. The air environment constantly tries to take the system to the room temperature, as shown in Fig. 4.14. As it is known, the farthest from the room temperature is the temperature perceived by the sensing probe the largest is the interference produced by the environment, since air micro-currents are created. This means that when the temperature perceived by the sensing probe is close to the room temperature, the influence of the environment is low. Otherwise, when temperature perceived by the sensing probe is far from the room temperature, the influence of the environment is larger, affecting the experimental signal evolution and thus producing a high level of noise.

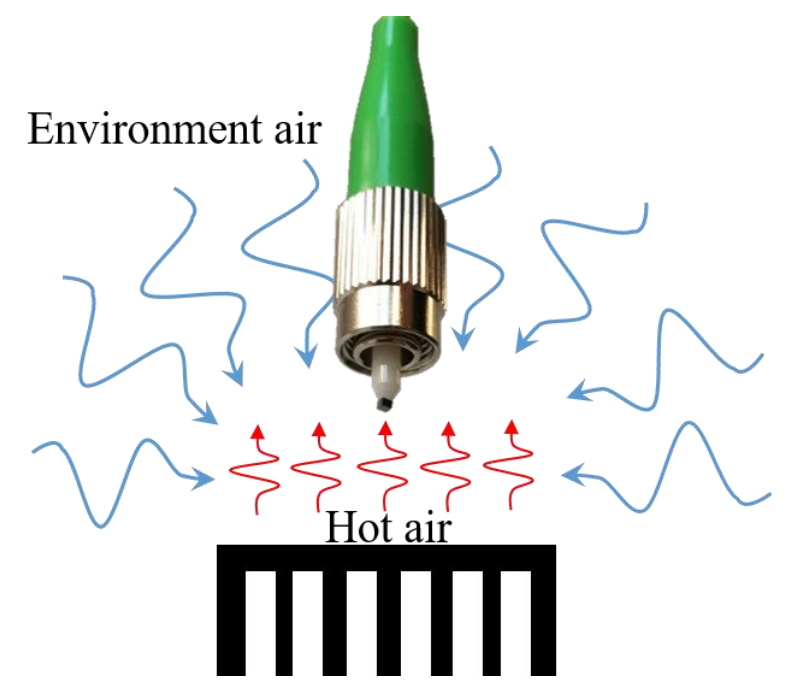

Fig. 4.14: Schematic illustration of the thermal influence of the environment on the sensing-in-air configuration system.

\subsubsection{Time response analysis}

In water environment, the observed time response was around 90 seconds. It is important to point out that such a time was determined by, firstly, the time needed by the Peltier cell to reach the required temperature (which was around 30 seconds) and, then, by the time needed by the water to achieve such temperature. This means that water took around 60 seconds to reach the required temperature. In this case, water acted like a second heatsink: as the heatsink warmed up and transferred the heat to the thermocouple, the water warmed up and transfer the heat to the sensing probe.

In air environment, a response time of around 30 seconds was observed, which is the same time needed by the thermal setup itself to heat up the thermocouple. Such result demonstrates that our temperature sensing probe was able to measure very small temperature variations in real-time. 


\subsection{Conclusions}

In this chapter, we have presented a novel FOS configuration for temperature sensing. The development of the sensing probe was based on the combination of an optical fiber and a PSMC. The PSMC sensor was obtained easily and rapidly.

The PSMC sensing probe behaviour was studied by measuring, in real-time, temperature changes in water and in air environments. In water environment experiments, the performance of the PSMC sensing probe was characterized, obtaining a sensitivity around 110 $\mathrm{pm} /{ }^{\circ} \mathrm{C}$ and a resolution in the $10^{-4}{ }^{\circ} \mathrm{C}$ range. In air environment experiments, it was observed that, first, for equal temperature changes, higher noise levels were measured at higher temperatures due to the temperature transmission dynamics through the environment and, then, the response was in real-time.

In conclusion, the experimental results obtained in both environments demonstrate that a temperature sensing probe able to both measure very small temperature variations in real-time and with a very high spatial resolution was developed. 


\section{Chapter 5}

\section{Experimental sensing using a porous silicon ring resonator}

\subsection{Introduction}

In chapter 2, we have seen that RRs are among the most employed photonic structures in the development of sensing devices and that a traditional planar wire based RR can reach a sensitivity around $160 \mathrm{~nm} / \mathrm{RIU}$. However, we have also seen that the performance of a traditional planar RRs is limited by the fact that only the evanescent field propagating outside of the photonic structure is exploited for sensing purposes, while the majority of the optical field distribution associated with the guided mode propagates within the structure itself. This issue is characteristic of all the traditional integrated photonic sensing structures.

In this chapter, we present a solution to this limitation, allowing to enhance the sensitivity of a traditional integrated photonic structure: a porous silicon based integrated photonic sensor. As a matter of fact, the PS platform allows the possibility of infiltrating the target analytes directly into the pores. In this way, not only the evanescent way is exploited for biosensing purposes, but also the field propagating in the core of the photonic structure, leading to a significantly enhancement of the sensitivity, as shown in Fig. 5.1. 


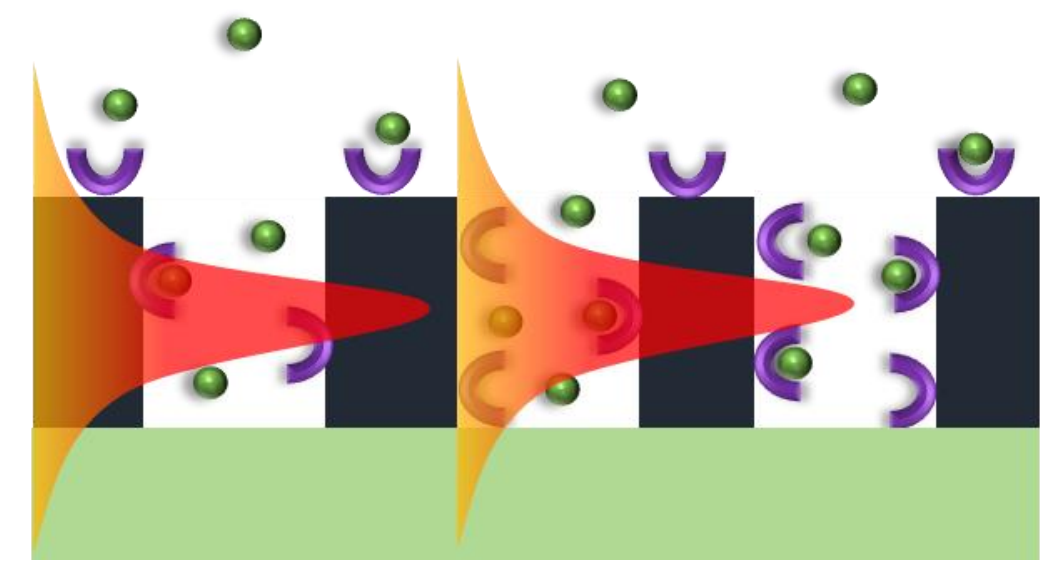

Fig. 5.1: Schematic illustration of the light-matter interaction occurring in the core of a PS structure.

In this chapter, we present the development of a porous silicon RR (PSRR) and the study of its sensing performance for transverse-electric (TE) and transverse-magnetic (TM) polarizations. In section 5.2, all the process to obtain the PSRR is exposed. In section 5.3, the experimental setup developed to characterize the sample is described. The experimental setup is presented in section 5.4. In section 5.5, we report the results of the experimental measurements. In case of each polarization, we measured the sensitivity for every resonance, which is determined by the optical field distribution of the PSRR resonant mode and its interaction with the medium filling the pores. Finally, section 5.6 contains the conclusions.

\subsection{PSRR fabrication}

\subsubsection{Structural approach}

The fastest and easiest strategy to develop a PSRR is the direct porosification of the silicon top layer of a SOI wafer. Furthermore, the presence of the oxide sublayer guarantees stability and robustness. However, this strategy presents a practical issue, which makes the PS fabrication more complicated, and a further limitation, which reduces the performance of the structure.

First of all, regarding the practical issue, the substrate of a SOI wafer is made of silicon oxide, which is an insulator. This makes not possible the electrochemical etching of the SOI wafer by using the techniques described in chapter 3 . To overcome this issue, a lateral contact connected with the silicon top layer is needed, as shown in Fig. 5.2, in order to allow the current to flow. However, this configuration is complicated to develop and, furthermore, it does not allow a homogenous porosity. 


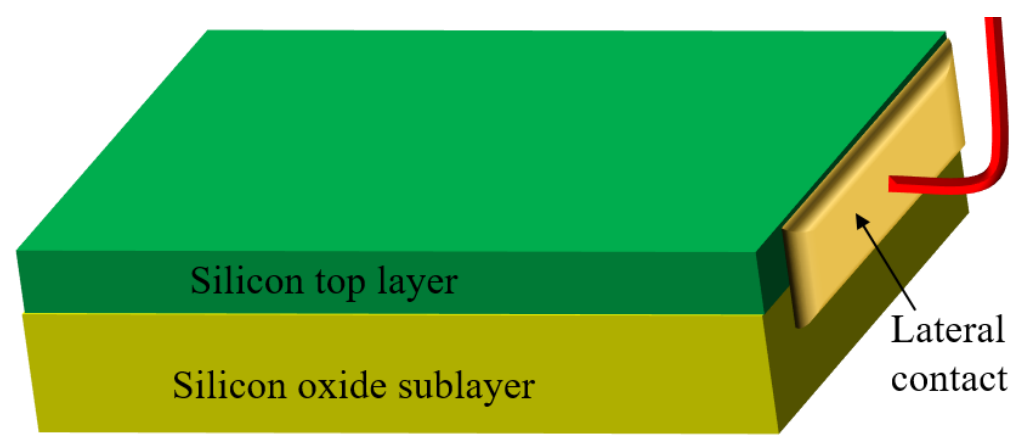

Fig. 5.2: Schematic illustration of the placement of the lateral contact connected with the top silicon layer of a SOI wafer.

Moreover, another limitation has to be considered. In the case in which the electrochemical etching of the silicon top layer can be carried out, the generated electrochemical field is not straight, but rather it is curved. This leads to the grown of curved pores, which interconnect among them causing a high lack of uniformity of the porosity and also, eventually, even the collapse of the structure.

Taking the importance of a high light confinement into account, in order to overcome these limitations, we chose another strategy: the fabrication of a PS double layer using a silicon wafer, as shown in Fig. 5.3. The RI profile of the PS double layer was selected aiming at having a strong light confinement in the top layer, where the photonic sensing structures was patterned. To reach this goal, the RI contrast between the top and bottom layers of PS needs to be as high as possible when water is infiltrated into the pores.

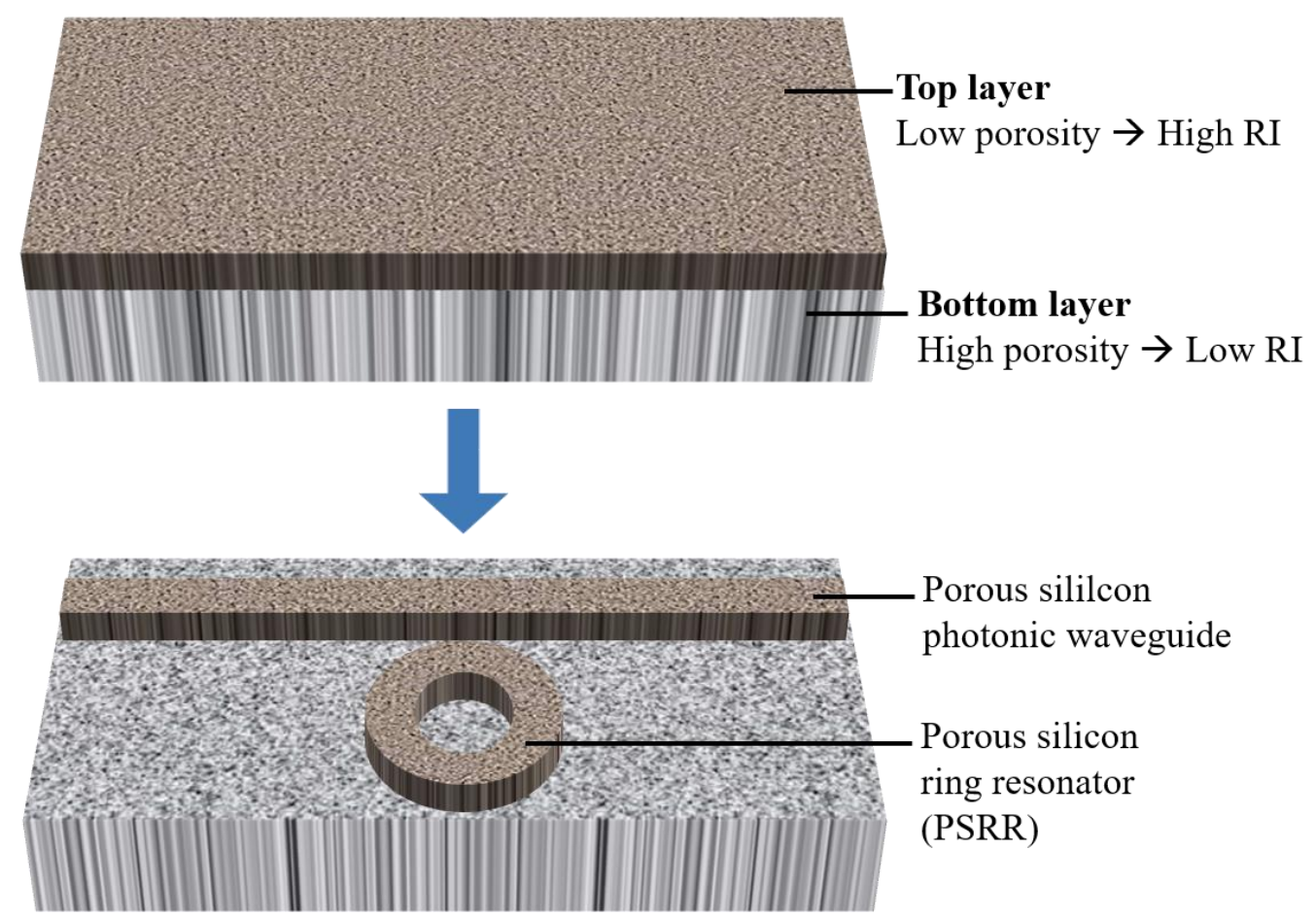

Fig. 5.3: Schematic illustration of the strategy used to obtain a PSRR. 
However, there is a limitation in the maximum reduction of the RI of the bottom layer since it implies increasing the porosity of this PS layer, what compromises the photonic structure stability. Taking these considerations into account, we selected porosities as high as possible, maintaining, at the same time, the stability of the double layer. For the top and bottom PS layers, porosities of $35 \%$ and $65 \%$ were selected, respectively. By using the Bruggeman equation, the RIs of the PS layers for these porosities were determined, being $n_{\text {top-air }}=2.56$ and $n_{\text {sub-air }}=1.7$, when considering the pores filled by air. Since, in our sensing experiments the pores will be filled by water, RIs $n_{\text {top-water }}=2.67$ and $n_{\text {sub-water }}=1.95$ were calculated. By using these RI values, several simulations were carried out using the software FemSIM in order to confirm the light confinement in the top layer when considering the RIs for a water environment, as shown in Fig. 5.4.

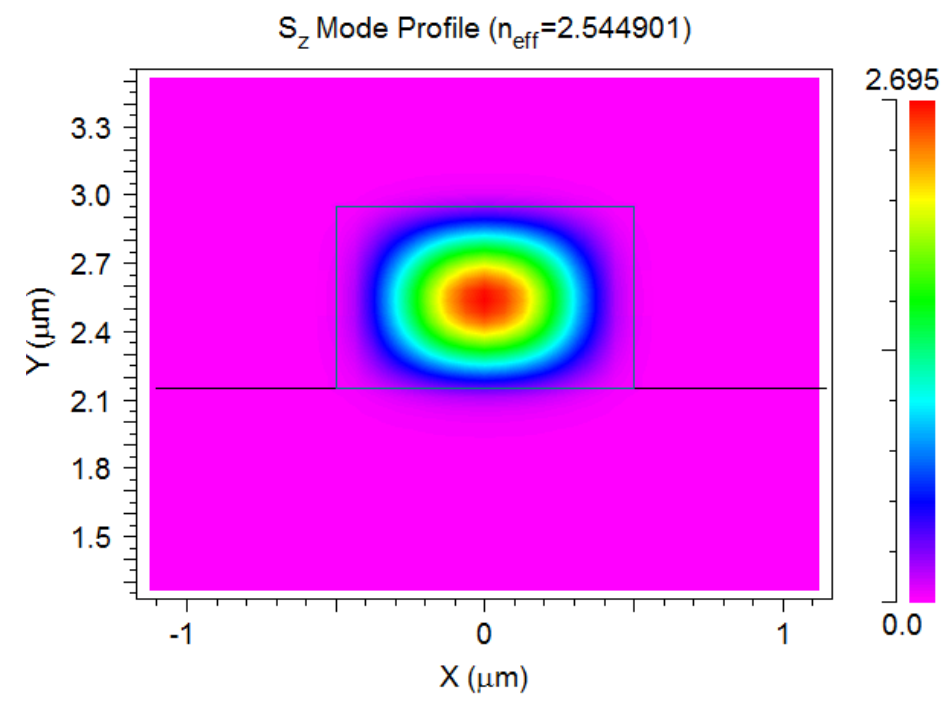

Fig. 5.4: Simulation of the optical propagation in the PS photonic structure. (height $=800 \mathrm{~nm}$ and width $=1000 \mathrm{~nm}$ ).

\subsubsection{Double layer fabrication}

The designed double-layered PS was fabricated by an electrochemical etching of a single-side polished boron doped (resistivity of $0.016-0.02 \Omega \cdot \mathrm{cm}$ ) $<100>\mathrm{Si}$ wafer with a thickness of 380-420 nm. The thickness of the silicon sample was almost half of those used for the fabrication of the PS multilayers in order to allow an easier cleaving of the sample, as it will be described in the next section. Just before the etching process, the silicon wafer was cleaned from possible organic residues using a piranha solution and then immersed into aqueous solution of $\mathrm{HF}(5 \%)$ to remove $\mathrm{SiO}_{2}$ from the surface. After each cleaning step, the wafer was rinsed in DIW. The top layer with high RI was formed by applying a current density of $5 \mathrm{~mA} / \mathrm{cm}^{2}$ for 100 seconds and the bottom layer of $45 \mathrm{~mA} / \mathrm{cm}^{2}$ for 110 seconds. The cleaning procedures and the electrochemical etching were performed at room temperature. The fabricated PS sample was washed with acetone, isopropyl alcohol and DIW to remove residual surface impurities. Finally, an $\mathrm{O}_{2}$ plasma was applied for 6 minutes, in order to improve the hydrophilicity of the PS surface. 
The fabricated PS sample was cleaved into two parts, as shown in Fig. 5.5: one intended to the physical characterization of the PS layers and the other one to the photonic structures fabrication.

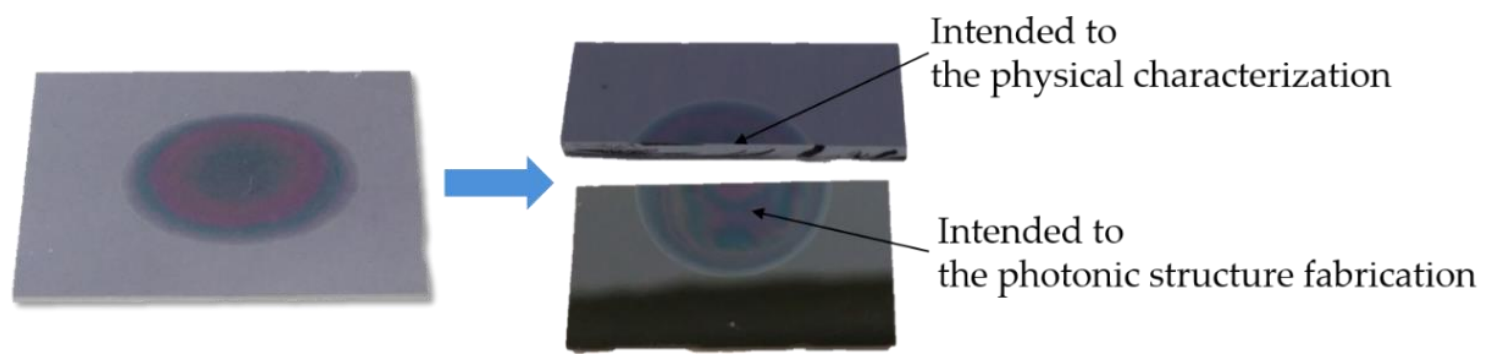

Fig. 5.5: Schematic illustration of the procedure to characterize, first, physically and, then, optically the PS structure.

Field emission scanning electron microscope (FE-SEM) was used to study the thickness and the uniformity of both the PS layers as well as the average pore diameter of the top PS layer. Figure 5.6 (a) shows a FE-SEM image of the cross section of the double-layered PS, where the different porosities of both layers are clearly distinguished. The measured thicknesses for the top and bottom PS layers were around $800 \pm 25 \mathrm{~nm}$ and $4350 \pm 150 \mathrm{~nm}$, respectively. Refractive indices of the double-layer were calculated from Fresnel coefficients under the Transfer Matrix Method approach using reflection measurements of the porous structure. In this respect, the refractive indices obtained were $\mathrm{n}_{\text {top-air }}=2.48$ and $\mathrm{n}_{\mathrm{lc}-\text { air }}=1.75$ for the air-filled pores, what corresponds to porosities of $38 \%$ and $63 \%$, respectively. These porosities are practically identical to those previously selected in the design phase, thus indicating a good control of the PS fabrication procedure. Figure 5.6 (b) shows a top-view FE-SEM image. The average pore diameter of the sensing structure was determined to be around $12 \mathrm{~nm}$.
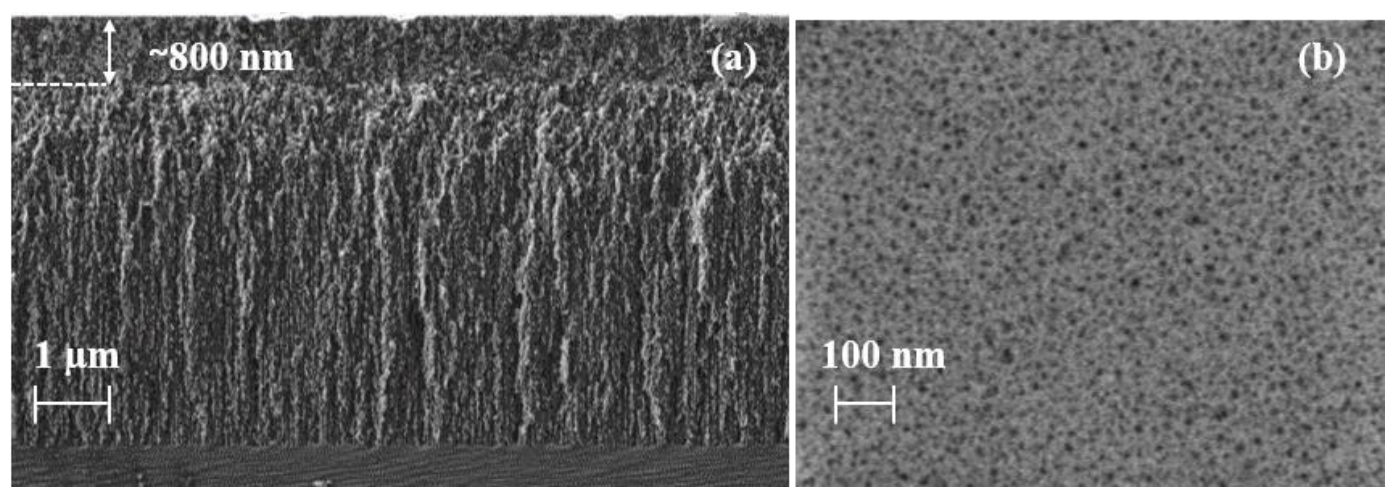

Fig. 5.6: FE-SEM images of (a) cross section and (b) top-view of the PS structure. 


\subsection{Ring resonator fabrication}

The design consisted of a $20 \mu \mathrm{m}$-radius RR coupled to a photonic waveguide, as shown in Fig. 5.7. The waveguide was composed by three section kinds: input (output) section, taper and coupling section. In this waveguide design, there were no grating couplers as in the design described in chapter 2. The input (output) section had a length of $2600 \mu \mathrm{m}$ and a width of $10 \mu \mathrm{m}$, in order to ease the butt-coupling characterization of the photonic structures. The tapers had a length of $1000 \mu \mathrm{m}$, in order to reduce (enlarge) gradually the width of the waveguide and connect the input (output) section with the coupling section. The coupling region had a length of 1000 $\mu \mathrm{m}$. The total length of the waveguide was $8200 \mu \mathrm{m}$.

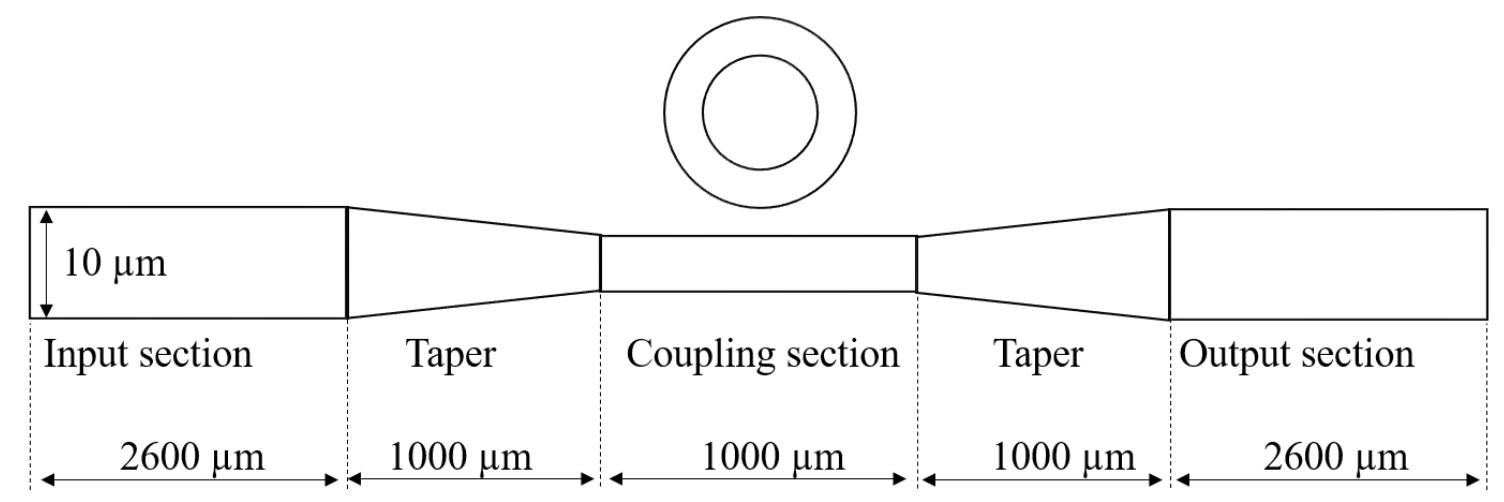

Fig. 5.7: Schematic of the basic design.

The chip design consisted of several sets of waveguides characterized by different widths of the coupling section and the RR waveguide (800, 1000 and $1200 \mathrm{~nm}$ ) and different coupling gaps between the coupling waveguide and the RR (150, 200, 250, 300 and $350 \mathrm{~nm}$ ), as shown in Fig. 5.8. 


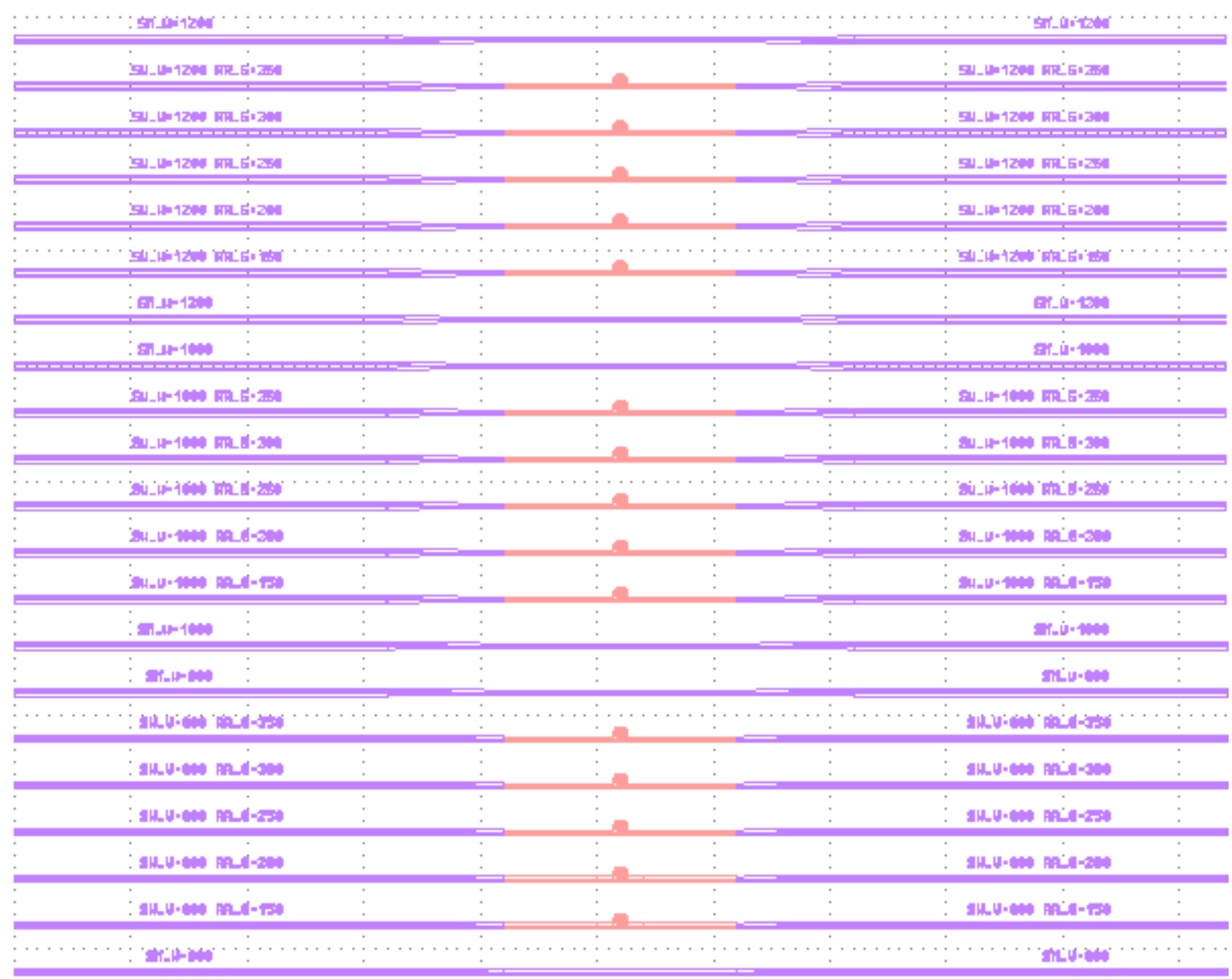

Fig. 5.8: Layout of the chip design.

A lithographic process was used to create the planar photonic structures in the top PS layer. The chip design was patterned into a PMMA positive resist layer using e-beam lithography with an acceleration voltage of $10 \mathrm{keV}$ and a dose of $\sim 87 \mu \mathrm{C} / \mathrm{cm}^{2}$. Then, after developing the resist, the pattern was transferred to the top PS layer by inductively coupled plasma (ICP) etching. In Fig. 5.9, the result of the fabrication process is shown.

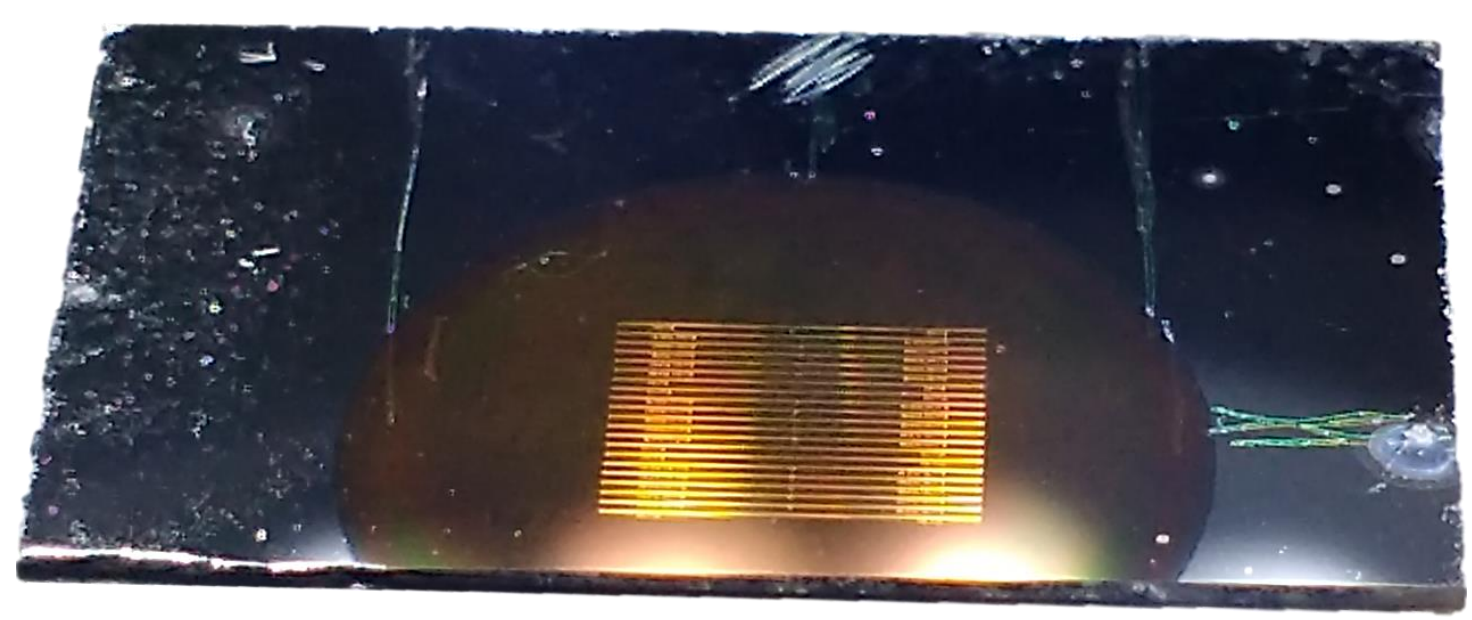

Fig. 5.9: Picture of the PS chip. 
Since there were not grating couplers in the photonic structures design, the fiber-chip vertical light coupling was not possible. In this case, in order to couple the light, horizontal coupling was used. To this aim, the sample was cut in the input (output) section along the dashed line shown in Fig. 5.10.

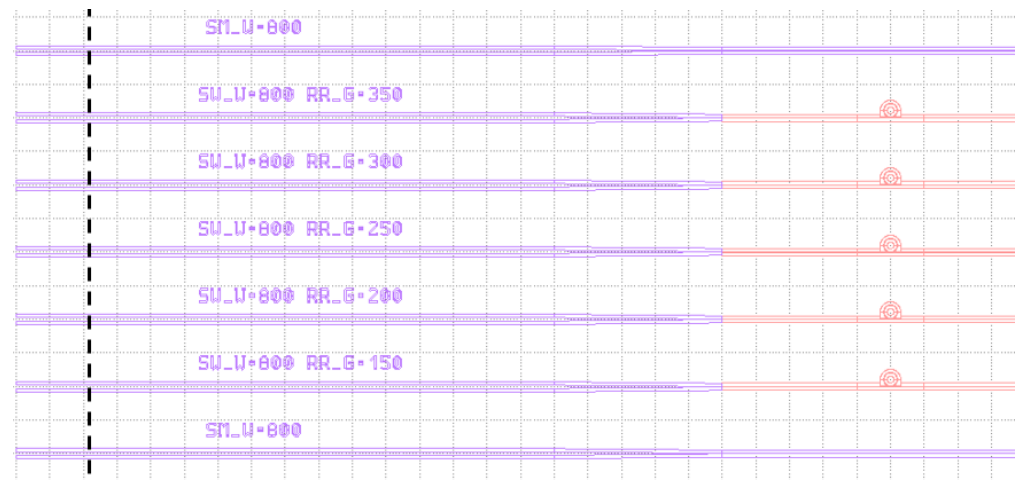

Fig. 5.10: Image of the layout with the dashed line along which the PS sample must be cut.

The cut of the sample can be achieved by means of an automatic dicing saw or by manual cleaving. Obviously, the cut carried out by using an automatic dicing saw allows more precision than the manual cleaving. However, the dicing saw cuts the sample without meeting the crystallographic orientation of the Si sample, causing the damages of the edge of the chip and, thus, the waveguide input, as shown in Fig. 5.11. In this optical microscope image, the edge of a testing chip is shown. It is possible to appreciate the irregularity of the cut direction and the damage provoked at the input-output of the waveguides. In this way, the fiber-chip light coupling cannot optimally take place.
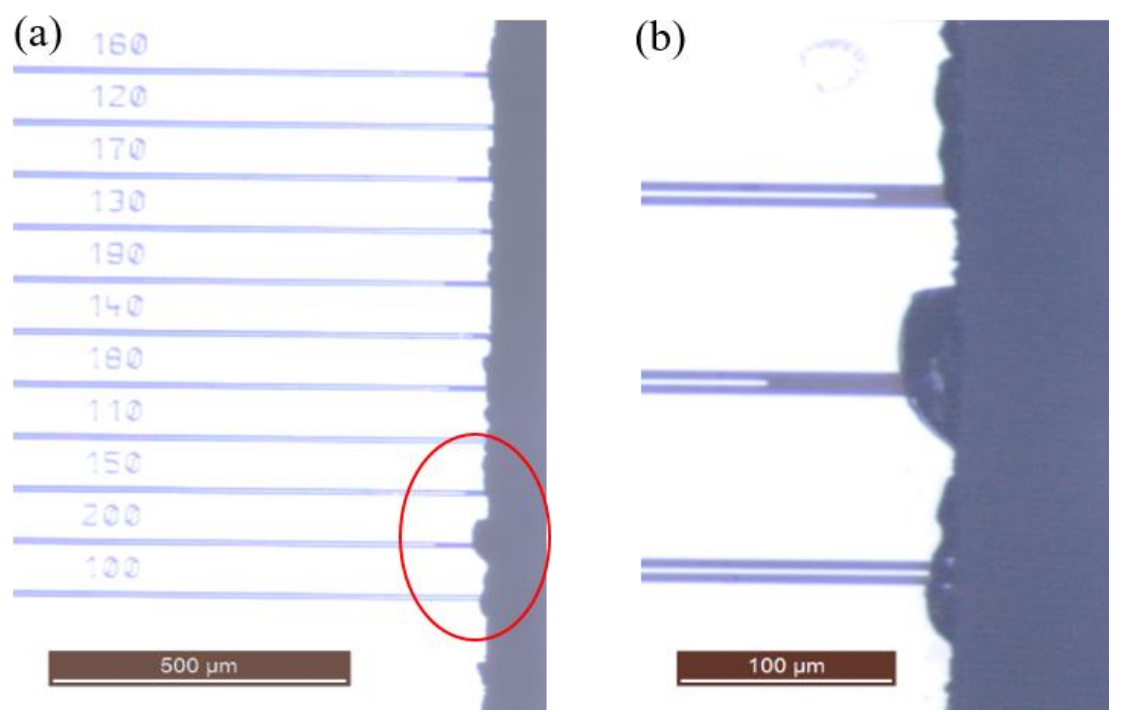

Fig. 5.11: (a) Optical microscope image of the edge of a testing sample after the cut process by means of the automatic dicing saw. (b) Zoom of the region highlighted with the red ellipse. 
On the contrary, the manual cleaving is based on the cut following the <100> crystallographic orientation of the Si sample, avoiding, in this way, the damage of the waveguides at the edge. Furthermore, the thinner thickness of the sample used in this case makes the manual cleaving more accurate and easier to carry out. For this reason, in this work, we selected $\mathrm{Si}$ samples of 380-420 nm of thickness and the PS chip was manually cleaved.

Before placing the PS photonic chip in the experimental setup and running the experiments, it was physically characterized in order to verify that all the fabrication process, including the PS double layer formation, the PS structure fabrication and the manual cleaving cut, was correctly executed. First of all, we checked the photonic chip fabrication by analysing it with an optical microscope. In Fig. 5.12, the PS chip in its final form is shown.
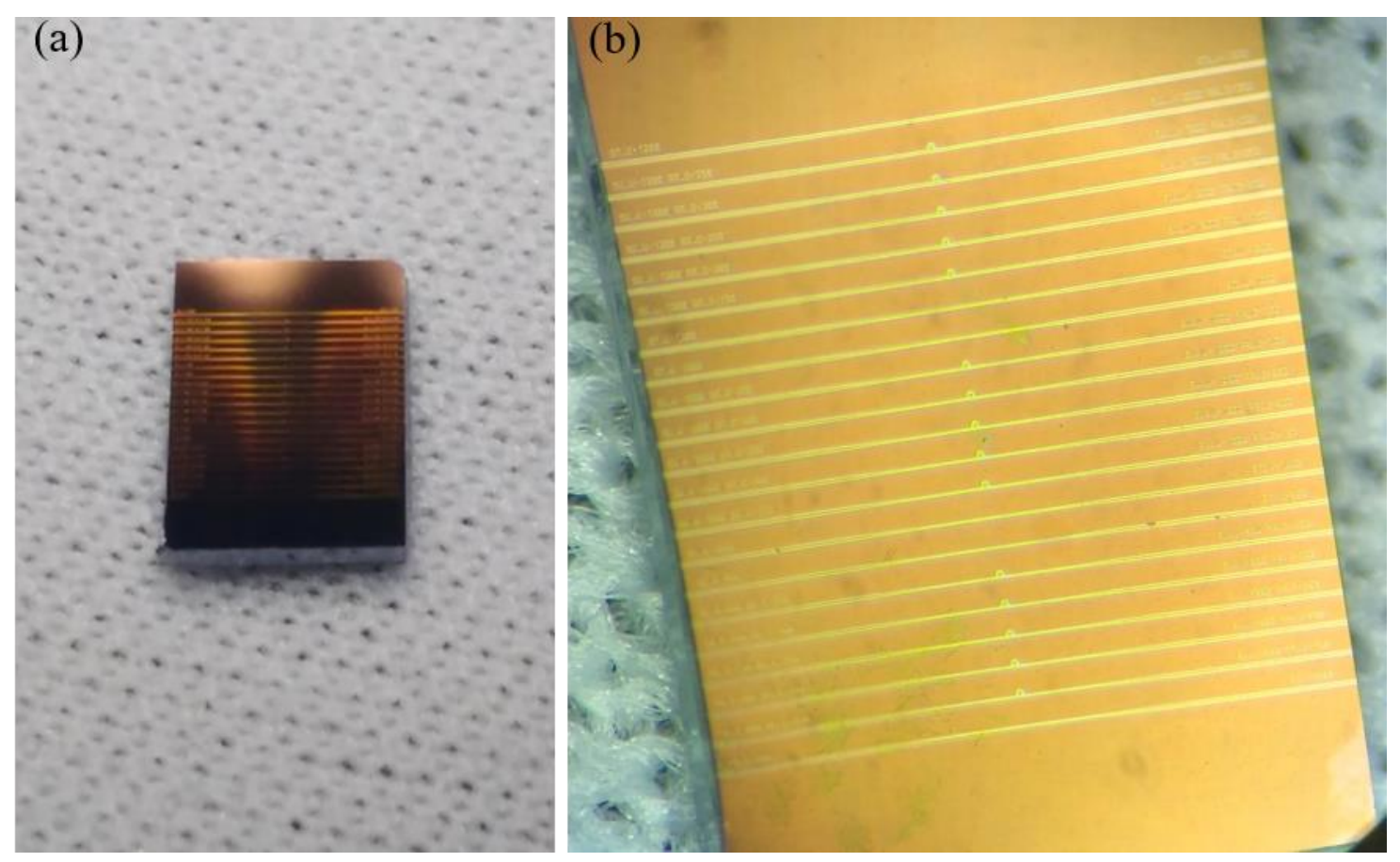

Fig. 5.12: (a) Picture and (b) optical microscope image of the definitive PS chip after the cleaving process.

Then, we checked the cleaving cut by analysing the PS chip edges. Just with these naked-eye images, it is possible to appreciate the almost perfectly linear cut along the perpendicular direction of the waveguides. In order to check the status of the input-output of the PS waveguides, the edges of the PS chip were analysed more in detail using a FE-SEM. In Fig. 5.13, FE-SEM images of the edge of an input-output waveguide are shown. It is possible to observe that the cut occurred straightly without damaging the waveguide. 

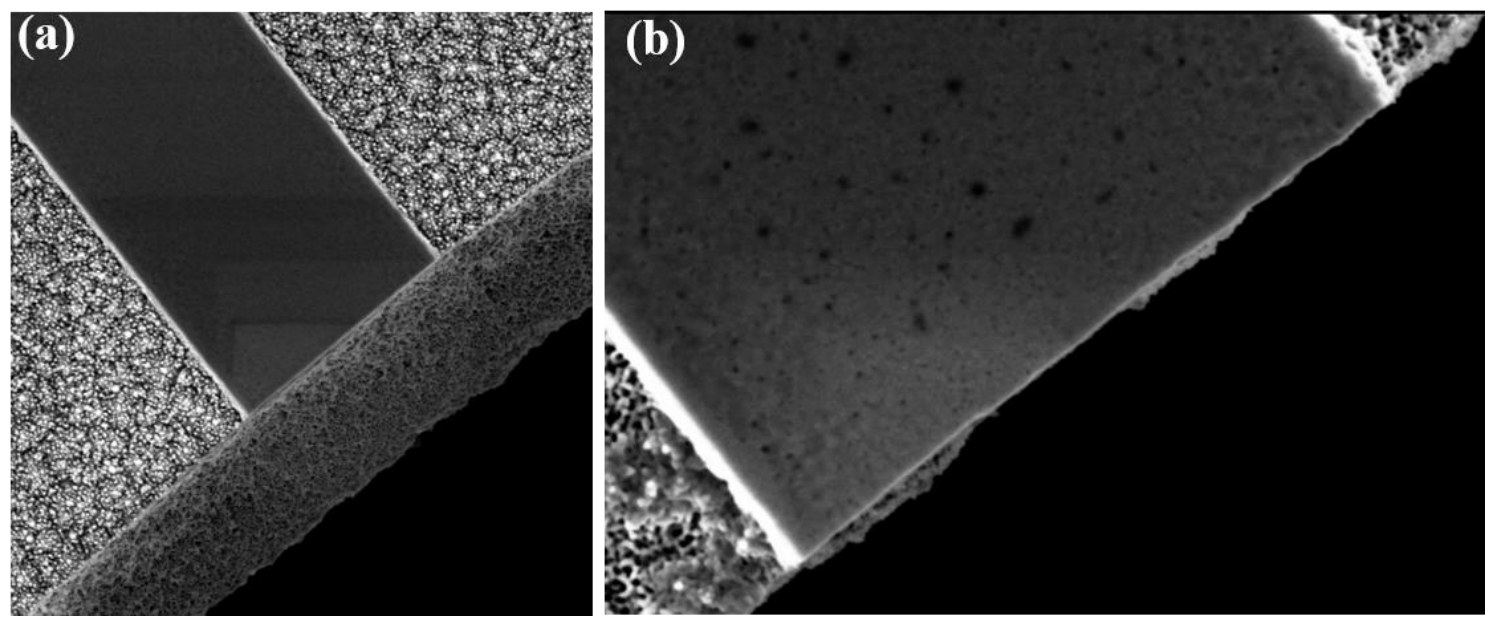

Fig. 5.13: (a) FE-SEM image of a waveguide input-output edge. (b) Zoom on the waveguide edge.

Afterwards, always by means of the FE-SEM, we analysed the section and the surface of the PS photonic structures. In Fig. 5.14, several FE-SEM images of the fabricated waveguides are shown. In Fig. 5.14 (a) the vertical orientation of the pores can be observed, thanks to the vertical cut of this sample edge. In Fig 5.14 (b), the sponge-like morphology of the PS and the boundary between the two PS layers can be clearly appreciated, thanks to a non-vertical cut of that sample edge. Figure 5.14 (c) shows the cross-section of a waveguide access and also its lateral side. In this image, we can observe the two porosities in both the dimensions.
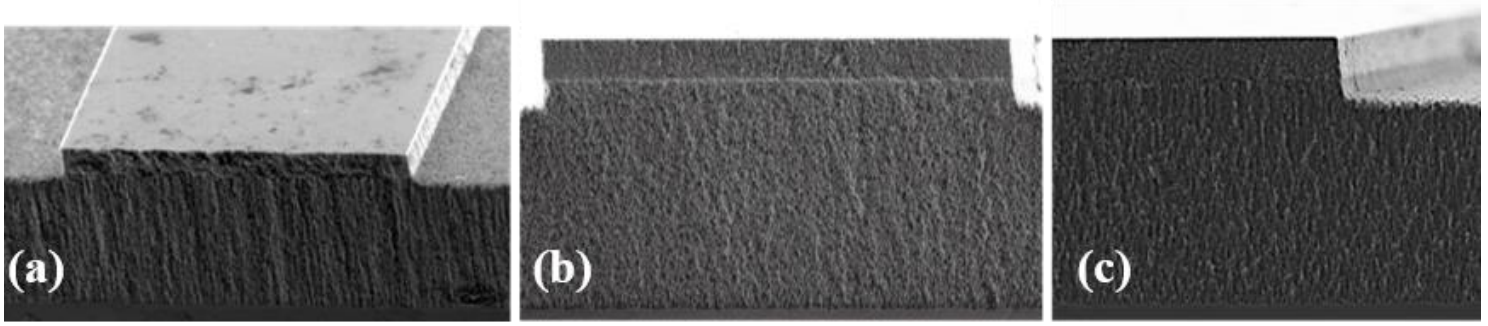

Fig. 5.14. FE-SEM image of the $10 \mu \mathrm{m}$-wide access waveguide: (a) $60^{\circ}$-sectional image; (b) cross-sectional image; (c) cross-sectional image with lateral view.

After the section, we analysed the surface of the chip. Figure 5.15 shows FE-SEM topview images of the PSRR. Figure 5.15 (b) shows the top and bottom PS layers. It is possible to appreciate the porosity difference between the two layers. 

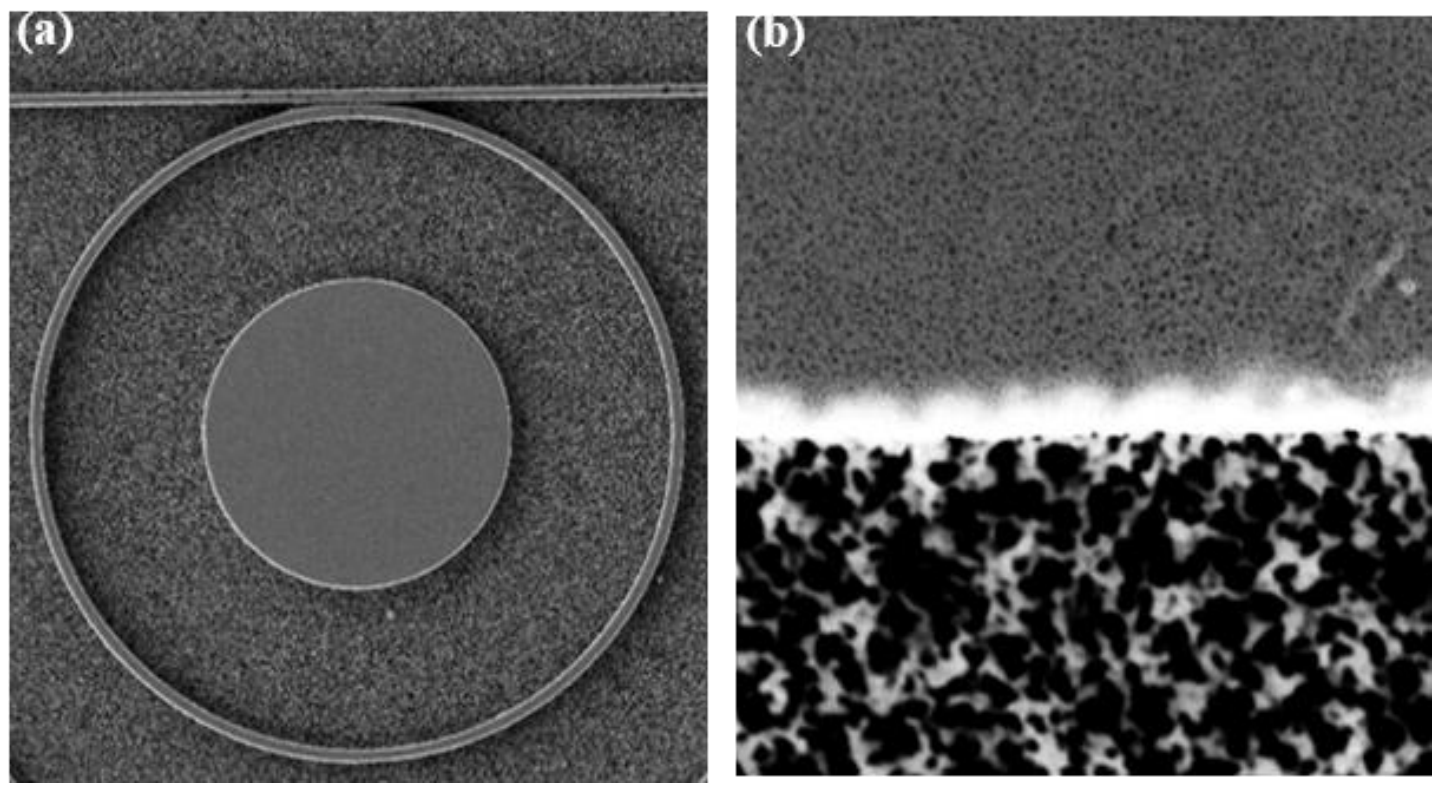

Fig. 5.15. FE-SEM top-view image of (a) the PSRR and (b) the top and bottom PS layers in the access waveguide region.

\subsection{Experimental opto-fluidic setup}

In this work, we used the same fluidic setup developed for the sample delivery described in chapter 2, composed by a PDMS microfluidic channel, input and output Teflon tubes, the syringe pump and the automatic switch. Also in this work, the PDMS-chip assembly was carried out by means of the flip chip tool.

Regarding the optical part, a new optical setup with a different configuration was developed. The optical setup consisted in a horizontal coupling interrogation platform, shown in Fig. 5.16 (a). Light was emitted by a continuous sweep tuneable laser (Keysight 81980A). The polarization of the input light was adjusted by using a polarization controller (Thorlabs FPC562). Input light was coupled to the access waveguides in the PSRR chip using a lensed fiber, as shown in Fig. 5.16 (b) and more in detail in Fig. 5.16 (c). Figure 5.16 (b) shows the opto-fluidic system, composed by the photonic chip and the PDMS microfluidic delivery system, placed on a holder, between the lensed fiber and an objective (20X Olympus Plan Achromat, 0.4 NA) used to collect the light coming out from the PSRR waveguide. Figure 5.16 (c) shows the alignment of the lensed fiber with the photonic waveguide in order to allow the horizontal light coupling. The light collected by the objective passed through a polarizer (Newport RM25A) in order to select the polarization. Then, light was divided by a 50-50 beam splitter cube. A 50\% of light was directed to a power sensor, which was connected to the power meter in order to monitor the power of the $50 \%$ of the output light during the alignment stage. The other $50 \%$ was directed to an infrared (IR) camera (Xenics Xeva-1.7-320) used to obtain the PSRR spectrum. 

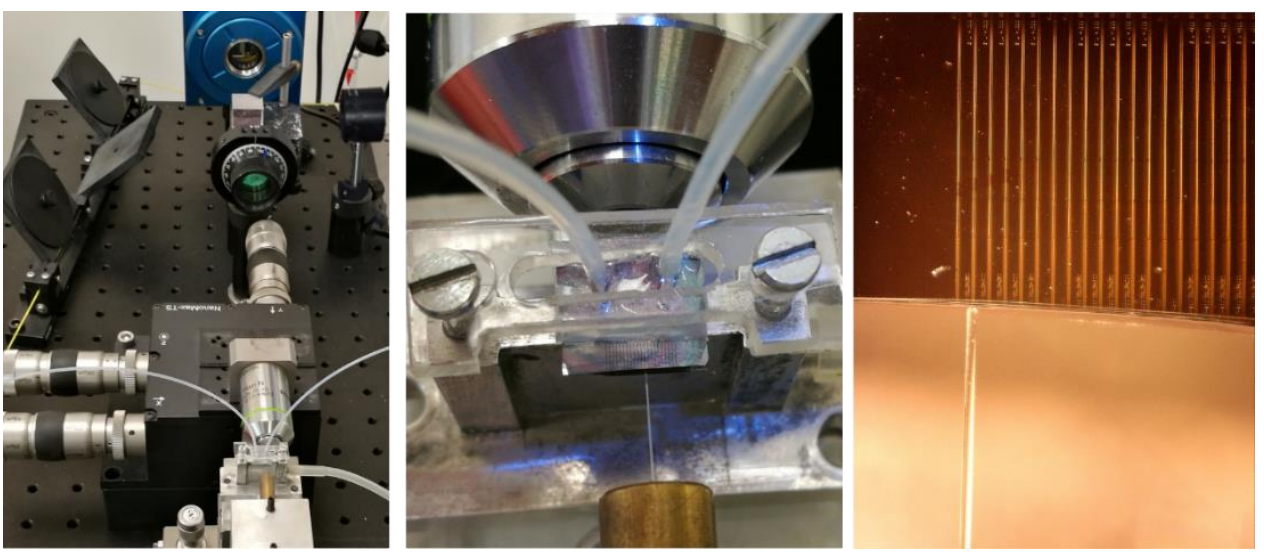

Fig. 5.16: (a) Picture of the horizontal coupling interrogation platform. (b) Picture of the chip-PDMS system placed in the optical setup. (c) Optical microscope image of the lensed fiber aligned with the waveguide.

The interrogation platform was controlled by using a software programmed in LabVIEW able to synchronize the continuous sweep of the laser with the image acquisition of the IR camera via a trigger signal, in order to obtain the spectrum of the photonic structure with the desired spectral resolution. The tuneable laser was swept from 1520 to $1620 \mathrm{~nm}$ with a sweeping speed of $10 \mathrm{~nm} / \mathrm{s}$, leading to a total time for each sweep of 10 seconds. The synchronization with the IR camera provided a spectral resolution of $20 \mathrm{pm}$. By means of the LabVIEW software, it is possible to display the light spot acquired with the IR camera at the $10 \mu \mathrm{m}$-wide output waveguide. The spot can be obtained when the lensed fiber is well aligned with the photonic waveguide. Figure 5.17 shows the LabVIEW software interface used to select the light spot and to acquire the PSRR spectrum. Finally, a Lorentzian fitting of each resonance was performed in Matlab to determine its position with a higher accuracy.

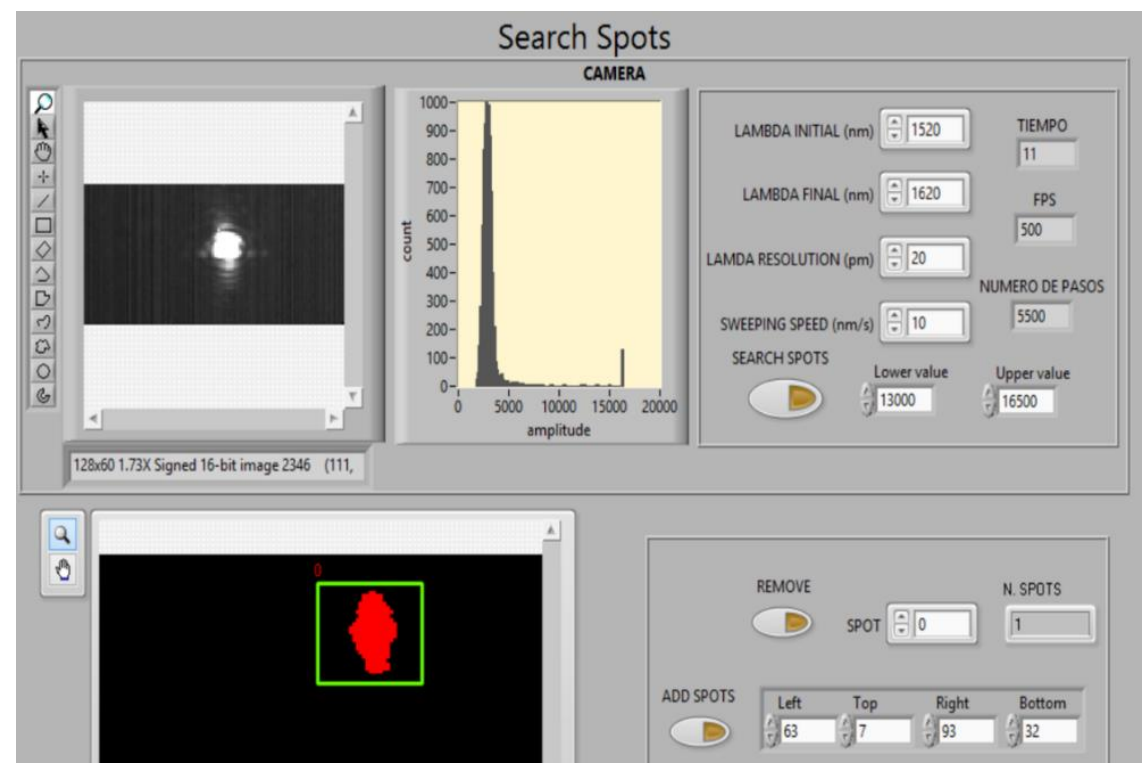

Fig. 5.17: LabVIEW software interface used to select the light spot and to acquire the PSRR spectrum. 
A schematic illustration of the final experimental opto-fluidic setup developed to characterize the PSRR spectrum and to carry out the sensing experiments is shown in Fig. 5.18.

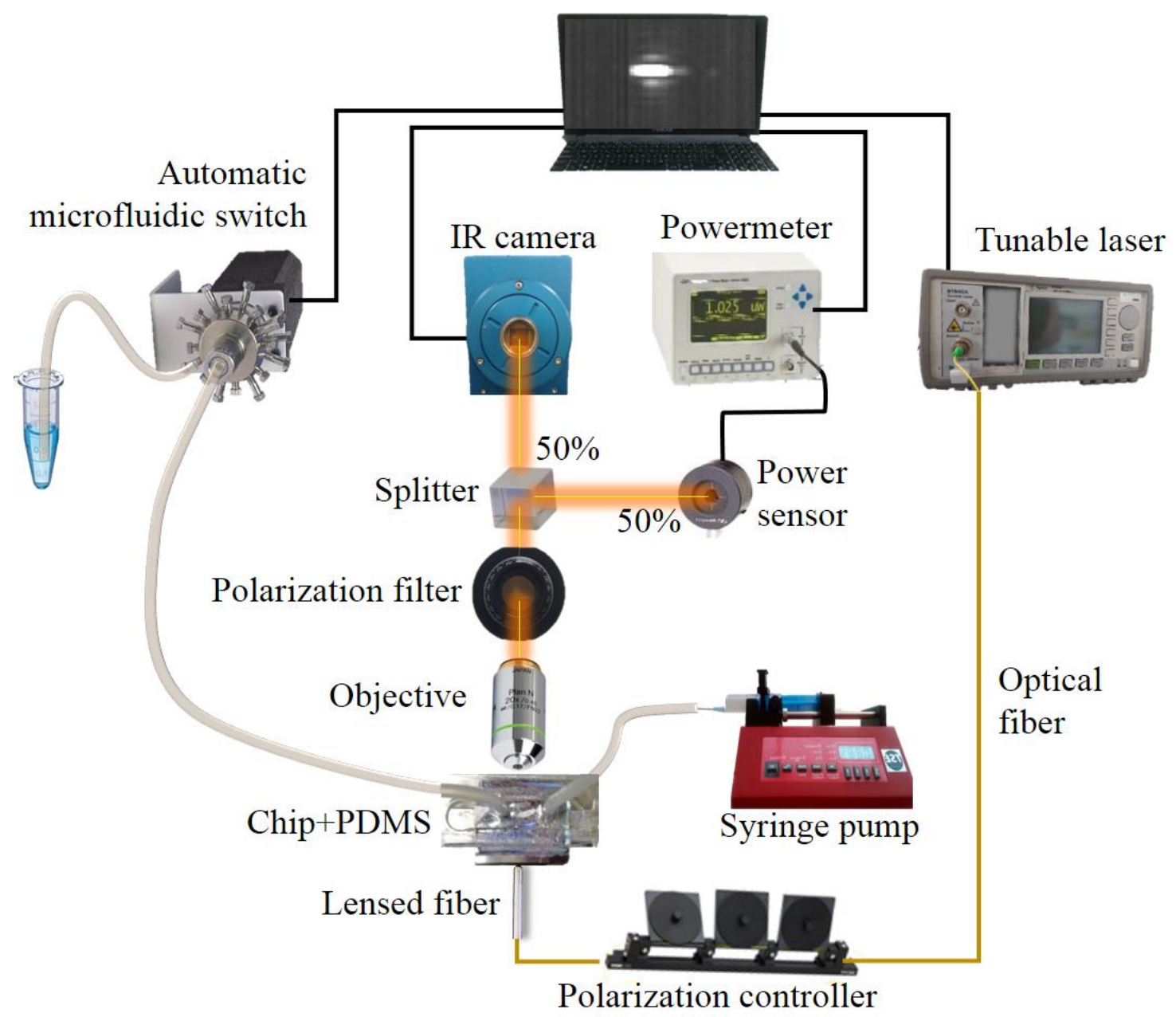

Fig. 5.18: Schematic illustration of the experimental opto-fluidic setup.

\subsection{Experimental results using a PSRR}

\subsubsection{PSRR optical characterization}

The first step of the optical characterization of the photonic structure consisted in the characterization of the light spot. This step is fundamental since it provides information regarding the light propagation inside the photonic structure. Once the spot is found, the image provided by the IR camera has to be optimized, since the more accurate the light spot image is the more accurate the obtained spectrum will be. To this aim, the output power has to be maximized and the spot focus has to be optimized. To maximize the output power, the fiber-waveguide alignment needs to be optimized in the order of $\mathrm{nm}$. To optimize the spot focus, the objective has to be correctly positioned. In Fig 5.19, the light spots obtained with the IR camera at the $10 \mu \mathrm{m}$-wide 
output waveguide for TE and TM polarizations are shown. It is possible to appreciate that a better confined mode is obtained for TE polarization, while a higher amount of light is going into the cladding for TM polarization.
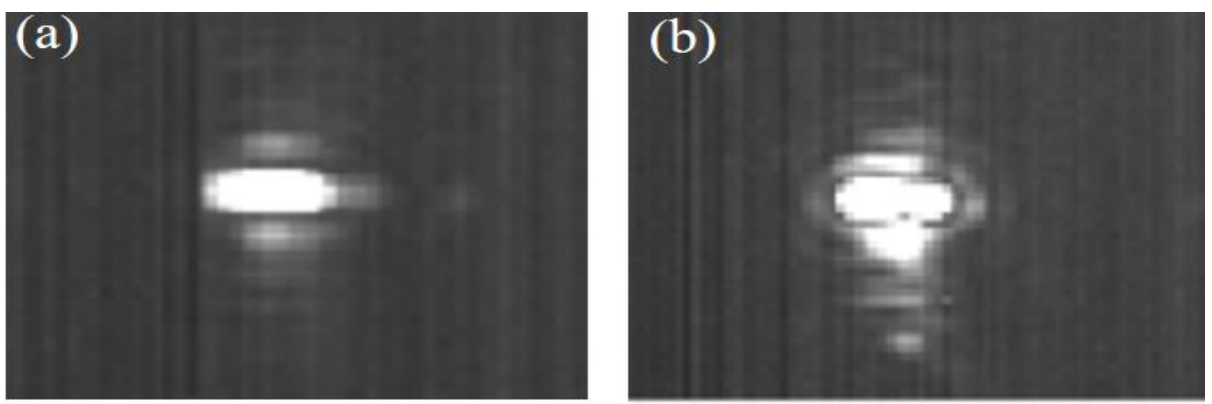

Fig. 5.19: Light spot for (a) TE and (b) TM polarizations.

Then, the spectra for all the PSRR configurations included in the photonic chip (i.e., different waveguide widths and coupling gaps) were characterized in water environment for the TE and TM polarizations. The best spectral responses in terms of resonances quality and optical losses were obtained for the configuration having a coupling waveguide width of $1000 \mathrm{~nm}$ and a coupling gap of $200 \mathrm{~nm}$. Figure 5.20 shows the measured TE and TM spectra in water environment for that PSRR configuration. The average quality factor of the measured resonances is quite reduced (453 and 512 for TE and TM polarizations, respectively) due to the higher losses that are produced in the PS-based waveguides compared to typical solid core ones.
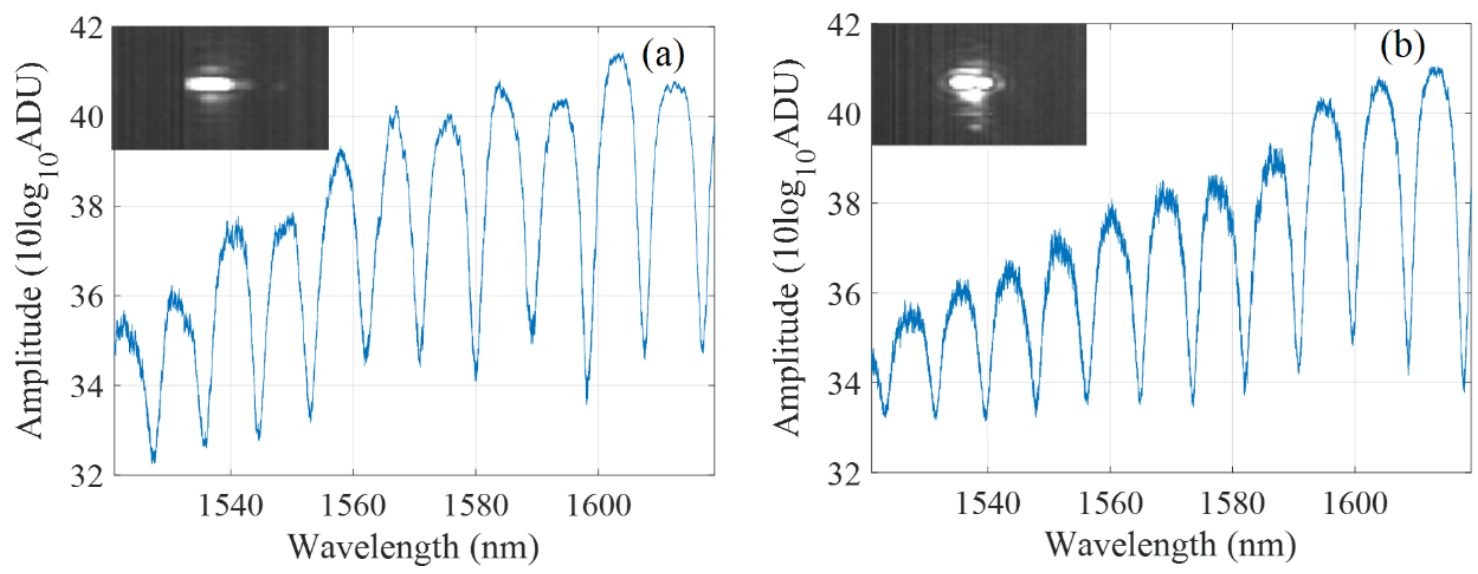

Fig. 5.20: Spectra of the PSRR for (a) TE and (b) TM polarization. The amplitude is represented in terms of the analogic-to-digital units (ADU) measured by the camera (in logarithmic scale).

The selected waveguide configuration was simulated using the software FemSIM in order to determine the modes existing on it for a water environment. Two fully propagating modes were obtained for that waveguide configuration for each polarization, while a third mode also begins to propagate for both polarizations. The field profiles of those modes are shown in Fig. 
5.21. From the optical profiles measured in the experiments, which were depicted in Fig. 5.19, we can see that only the fundamental mode is properly excited and propagated for TE polarization, while higher order modes are excited and propagated for TM polarization, leading to a higher delocalization of the optical field.
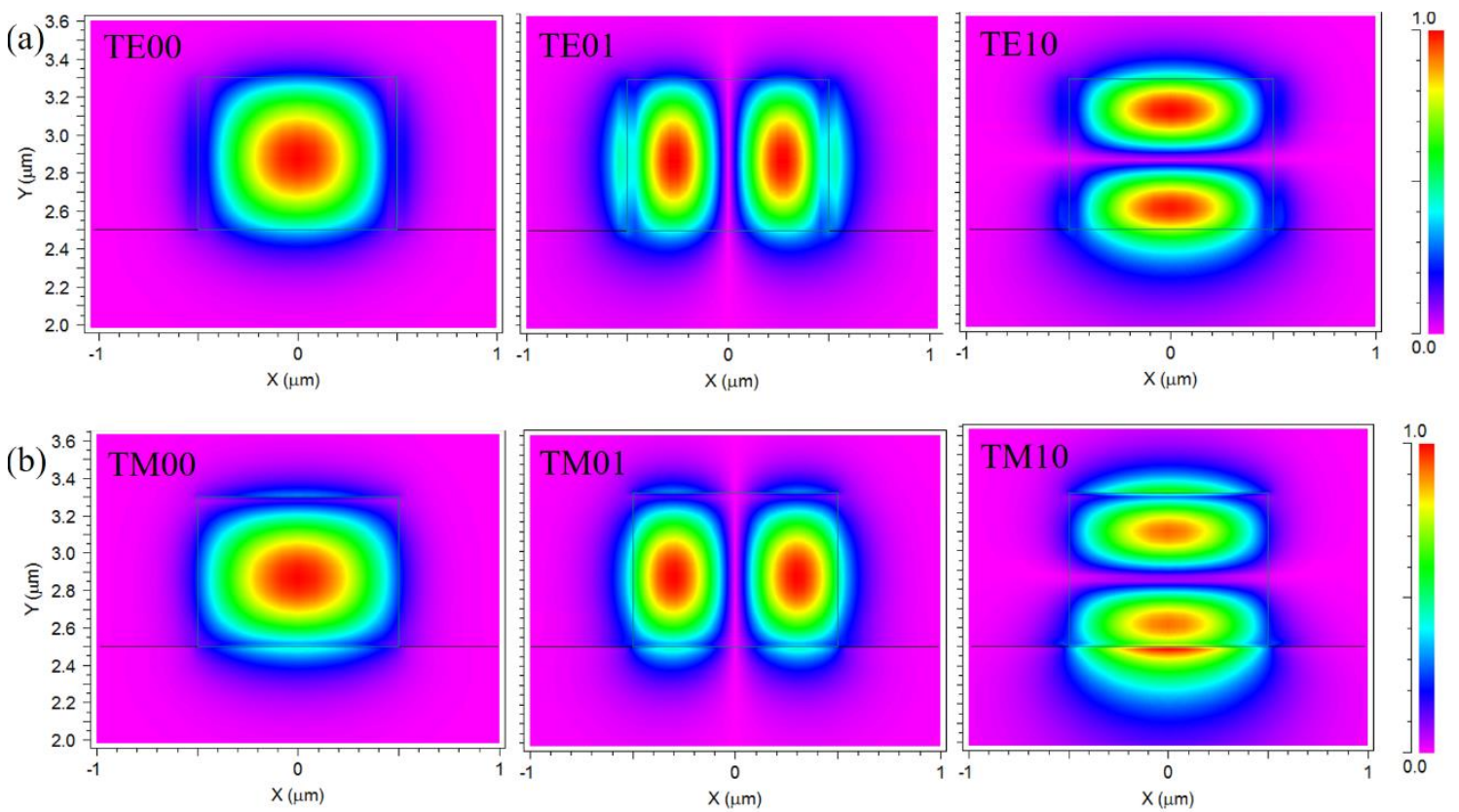

Fig. 5.21: Simulations of the optical modes propagating in the selected PS waveguide configuration (height $=800 \mathrm{~nm}$ and width $=1000 \mathrm{~nm}$ ) for (a) TE and (b) TM polarization. The modes are ordered from left to right.

\subsubsection{Sensing results}

Several RI sensing experiments were performed to determine the sensitivity of the PSRR photonic structure. To this aim, different ethanol-water dilutions were flowed over the sensor while the transmission spectrum was continuously acquired in order to determine the shift of the PSRR resonances. The ethanol (EtOH) concentrations in DIW used in the experiments were $10 \%, 5 \%$ and $1 \%$, which correspond to RI changes of $6.6 \cdot 10^{-3}, 3.3 \cdot 10^{-3}$ and $6.6 \cdot 10^{-4}$ RIU respect DIW, respectively. The three ethanol concentrations were flowed from the highest to the lowest. DIW was flowed before and after each concentration in order to have a baseline as reference. All the experiments were carried out for TE and TM polarizations.

Figure 5.22 (a) and Fig. 5.22 (b) show the time evolution of all the PSRR resonances within the sweeping range for TE and TM polarizations, respectively. The average sensitivity of the PSRR sensor (i.e., for all the resonances within the measured range) was around $350 \mathrm{~nm} / \mathrm{RIU}$ for TE and $320 \mathrm{~nm} / \mathrm{RIU}$ for TM when considering the whole range of refractive index variations that has been measured. The maximum sensitivity value was around $380 \mathrm{~nm} / \mathrm{RIU}$, provided by the resonance located at $\sim 1607.5 \mathrm{~nm}$ for TE polarization, whose quality factor was 513 . This 
sensitivity value was more than twice higher than the sensitivity achieved for a traditional SOI RR resonances within the measured wavelength range being tracked. The average noise level measured was around $0.1 \mathrm{pm}$, as it can be observed in the inset in the inset of Fig. 5.22 (a). This led to an estimated limit of detection of $2.7 \times 10^{-7}$ RIU.
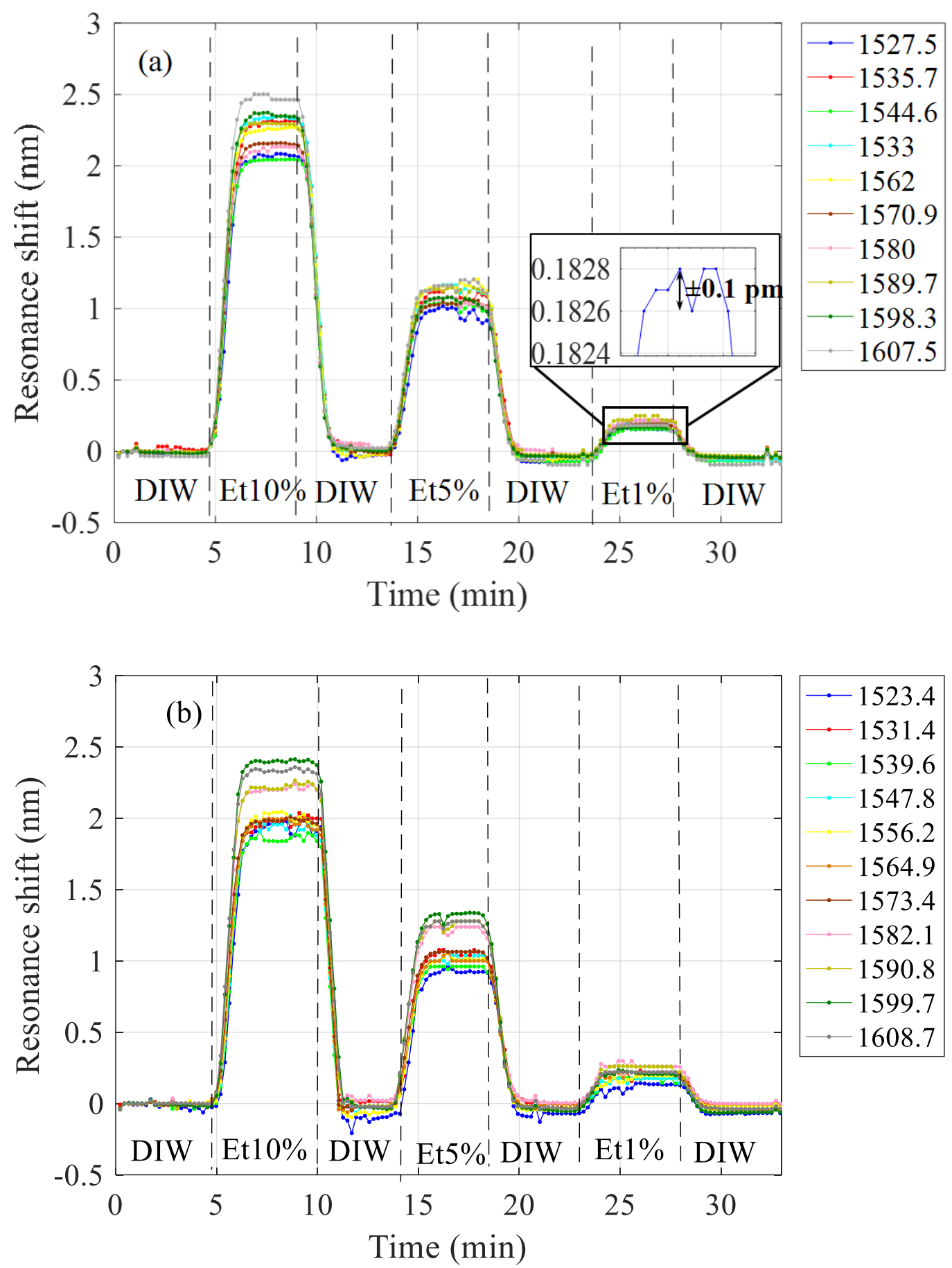

Fig. 5.22: Wavelength shift time evolution of all the resonances for (a) TE polarization and (b) TM polarization. The different colours indicate the different resonances of the PSRR. 
Figure 5.23 shows the spectral shift measured for each resonance for both polarizations. In this figure, an oscillatory-like behaviour can be observed as we move towards higher order resonances.

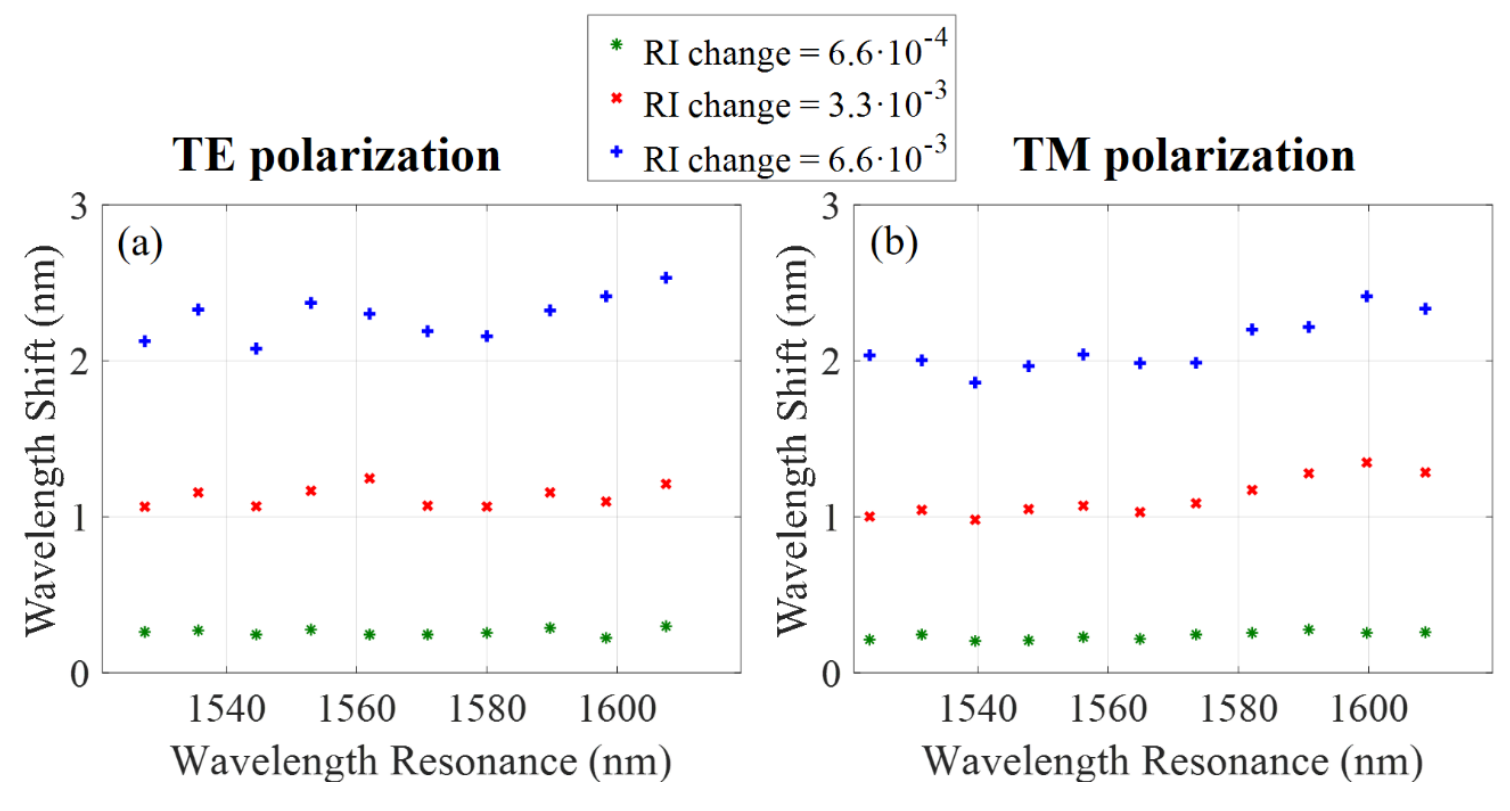

Fig. 5.23: Wavelength shift of each resonance for each RI variation for (a) TE and (b) TM polarizations.

\subsection{Results analysis}

In Fig. 5.24, the sensitivity curves of each resonance for TE and TM polarizations are shown. As it can be observed, the sensitivity behaviour of the PSRR sensing structure was not totally linear. In fact, the sensor exhibited a higher sensitivity for lower RI variations, specifically, for a RI variation of $6.6 \cdot 10^{-4}$ RIU corresponding to the EtOH $1 \%$ concentration. Consequently, considering a RI variation of $6.6 \cdot 10^{-4} \mathrm{RIU}$, the highest sensitivity provided by the PSRR sensor was obtained with a value of $439 \mathrm{~nm} / \mathrm{RIU}$ for the TE resonance located at $\sim 1607.5 \mathrm{~nm}$.
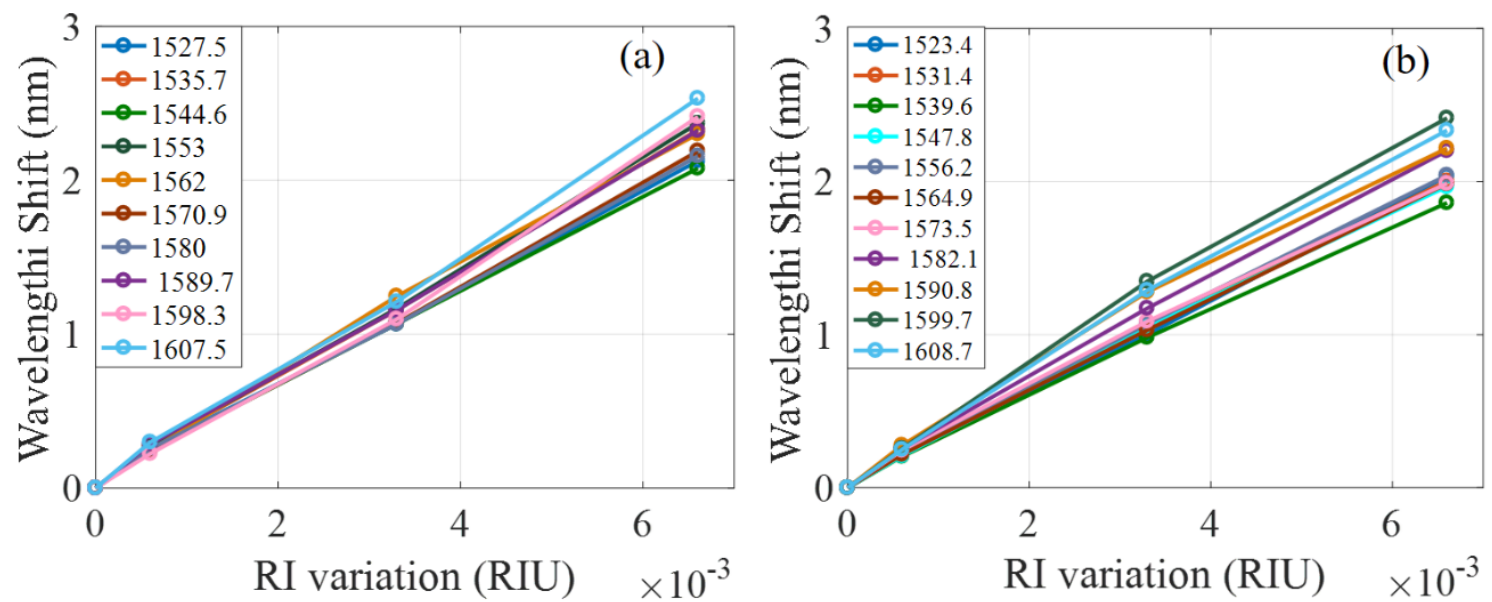

Fig. 5.24: Sensitivity curve of each resonance for (a) TE and (b) TM polarizations. 
This is related with the non-linear variation of the RI of the top porous layer that is produced when changing the RI of the solution infiltrated into the pores, as it is shown in Fig. 5.25. We can see that a higher variation of the effective refractive index of the top layer is obtained for the $\mathrm{EtOH} 1 \%$ concentration than for the $\mathrm{EtOH} \mathrm{5 \%}$ and 10\% concentrations. For this reason, the PS structure showed a higher sensitivity for such small EtOH concentration.

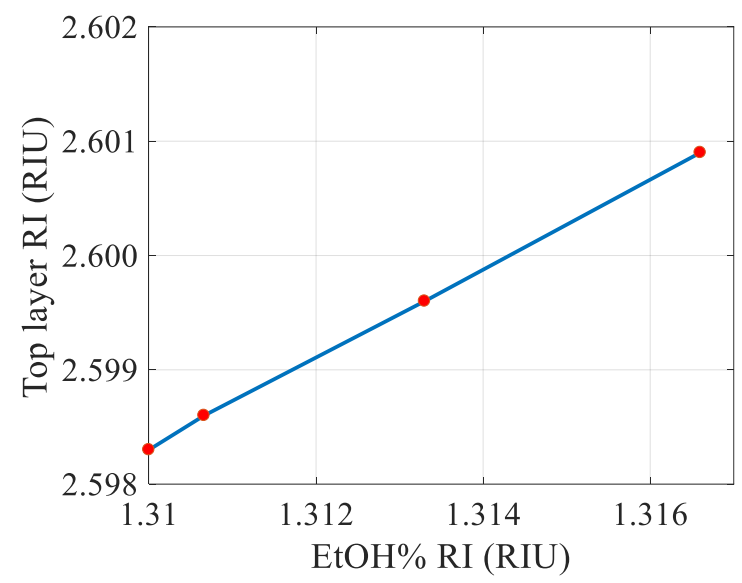

Fig. 5.25: Top PS layer RI variation as a function of the EtOH solution RI

In the sensing experiments, the resonances presented a similar behaviour for both polarizations. This was related to the fact that the sensing occurs mainly inside the guiding structures, where the field distribution is similar in both cases. However, a higher sensitivity was obtained for TE polarization resonances. This was due to a better light confinement for TE polarization, as it was shown in the spot images presented in Fig. 5.19. Since using our PS sensor the sensing event occurs inside the structure where the field is propagating, the better light confinement leads to a higher light-matter interaction. This is the opposite to what typically happens for evanescent wave sensors, where TM modes are generally more sensitive because of the presence of a higher amount of evanescent field over the top surface of the structure. In fact, using a PSRR, the sensitivity obtained in these experiments was considerably higher than that achieved for traditional SOI RRs. As it was reported in chapter 2, the highest sensitivity exhibited by a traditional planar RR was $160 \mathrm{~nm} / \mathrm{RIU}$, whereas in this work the sensitivity value was more than twice that value. The sensitivity obtained using the PSRR is also higher than that reported for other RR configurations as those based on the slot waveguide, where values up to $298 \mathrm{~nm} / \mathrm{RIU}$ have been measured. Additionally, different shifts have been obtained for each resonance of the PSRR (for each polarization). This sensitivity variation indicates that a different interaction between the resonance optical modes and the target substance is taking place, which might be determined by the local variations of the pores properties and distribution of the fabricated PS layer where the RR is created. Besides this oscillatory-like behaviour, the average shift also increases as a function of the wavelength, due to the larger evanescent field of the modes as wavelength is increased. 


\subsection{Conclusions}

In this chapter, we presented the enhancement of the sensitivity of traditional photonic structures using PS as material platform. More in detail, this chapter was focused on the development of a porous silicon ring resonator and its use for RI sensing. The PSRR was developed thanks to the formation of a PS double layer with a high RI contrast and its sensing performance was characterized both for TE and TM polarizations. The characterization was performed by monitoring in continuum the evolution of the sensing structure spectrum. The experimental results indicate that the sensitivity of the PSRR was slightly better for the TE polarization than for the TM polarization. This is because the sensing occurs in the core of the structure, where the TE polarization plays a major influence due to the better confinement of the light in the photonic structure. Working with such polarization, a sensitivity of $439 \mathrm{~nm} / \mathrm{RIU}$ was achieved for the detection of low RI variations. The higher light-matter interaction that is produced due to the fact that the sensing occurs directly inside the structure, together with the possibility of immobilizing the bioreceptors on the inner surface of the pores, make PS a suitable platform for the development of new biosensing devices exhibiting a high sensitivity 


\section{Chapter 6}

\section{Conclusions and future lines}

\subsection{Conclusions}

In this PhD Thesis, we have travelled a journey that began with the experimental study of more traditional photonic sensing platforms and evolved to the development of novel configurations based on porous silicon. These configurations solved the limitations of traditional photonic sensing structures and turned to be more sensitive and also cheaper, easier and quicker to develop.

We started our research using traditional photonic sensing structures for the biodetection of target analytes. As a model of photonic sensing structure we used a SOI RR, since this sensor is probably the most commonly employed photonic structure for the development of biosensing devices due to its simplicity, versatility and multiplexing potential. The aim was to demonstrate the crucial role of the biofunctionalization in biosensing applications. BSA was used as a target analyte model. To improve the BSA detection, we focused on the improvement of the antiBSA immobilization process. To this aim, the sensitivity to that antibody needed to be 
enhanced. For this reason, we biofunctionalized the sensor surface with an intermediate protein A layer. Thanks to this approach, we were able to enhance the sensitivity for antiBSA and, thus, to achieve a LOD of the antiBSA total mass of $60 \mathrm{ag}$. This led to an experimental LOD of BSA of $1 \mathrm{ng} / \mathrm{ml}$.

However, the sensitivity values previously mentioned were not maximized, since the sensitivity of SOI structures is limited by the fact that only the evanescent field interacts with the target analyte to be detected. Hence, we re-directed our study and our research to the development of a new platform to be used for the fabrication of photonic sensors with higher sensitivities. Such a platform was the PS. The reason why we chose PS is due to the several advantages provided by such a material. The main advantage is the possibility to use the inner surface of the pores for sensing purposes, leading to maximize the light-matter interaction and, thus, the sensitivity of the photonic structure. Furthermore, the development of PS structure is cheap, quick and easy to carry out.

PS photonic structures used in sensing applications are mainly based on refractivity. Among them, the most important sensors are based on reflectivity. This led us to the fabrication of sensing structures based on Bragg reflectors, which are probably the most used PS structures, and their use for RI variation sensing. Since the spectrum of a Bragg reflector is characterized by the present of a photonic reflectance band, we used a power sensing technique to characterize the performance of the structure. Here, we faced an issue related with the dependence of the power sensitivity with the spectrum slope, which was translated into a non-linear relation with the perceived refractive index variation. Instead, the sensing technique based on tracking a given spectral feature provided a linear sensitivity. For this reason, we re-directed our study to the fabrication of PSMCs and their use for RI detection. This kind of structure was found to be very easy and rapid to fabricate. Since this structure provides a very narrow resonance peak in the spectrum, we were able to characterize the PSMC performance by using the wavelength based sensing technique. With this photonic sensor, we achieved an outstanding sensitivity of around $1000 \mathrm{~nm} / \mathrm{RIU}$ and an experimental LOD of $6.6 \cdot 10^{-7} \mathrm{RIU}$.

Since temperature monitoring is crucial in several fields, we have also focused our attention on temperature sensing in order to contribute also in this field. Among temperature sensors, the most employed are FOSs. However, this kind of sensors present several limitations, owing to the use of the fiber itself as sensing element. This leads to a complex fabrication of the photonic structure into the optical fiber and to a limitation of the temperature sensitivity, since the optical fiber is made of silica, which presents a low TOC. Within this context, we took advantage of the PSMC to overcome these issues by developing a novel fiber-optic temperature sensor probe, as a result of the combination of an optical fiber and the PSMC itself. Our sensing probe 
was easy, quick and cheap to develop. By employing this PS based FOS, we achieved a temperature sensitivity of around $110 \mathrm{pm} /{ }^{\circ} \mathrm{C}$, against the $10 \mathrm{pm} /{ }^{\circ} \mathrm{C}$ of a standard FBG based FOS,

with a LOD of $6.3 \cdot 10^{-4}{ }^{\circ} \mathrm{C}$. In addition, thanks to this sensing configuration, we were able to detected such extremely low temperature variations in real-time and in a very specific point.

Finally, in order to demonstrate the advantage of using PS as material platform and, thus, to overcome the limitations of traditional SOI photonic sensing structures, we developed a PSRR and studied its performance, closing, in this way, the loop of this PhD Thesis. In order to obtain the PSRR, first, we fabricated a high porosity and high RI contrast PS double layer with a profile similar to that of SOI platform. The high porosity was needed to enhance the sensitivity, whereas, the high RI contrast to confine the light in the photonic structures. Then, in the high RI top layer, the photonic structures were fabricated. The light was well confined in the waveguide and the PSRR was optically characterized for TE and TM polarizations. For TE polarization, light was better confined, what led to a sensitivity higher in such a polarization. Thanks to this sensing platform, we were able to obtain a sensitivity of $439 \mathrm{~nm} / \mathrm{RIU}$, against the $\sim 160 \mathrm{~nm} / \mathrm{RIU}$ of a traditional planar SOI RR, and a LOD of $2.27 \cdot 10^{-7}$ RIU.

In conclusion, we have demonstrated the development of high sensitivity photonic sensing structures based on porous silicon. It has to be highlighted the design and the development of four different experimental setups, each of them being specially developed for each sensing structure.

\section{2. $\quad$ Future lines}

Starting from the results achieved in this $\mathrm{PhD}$ Thesis, it is possible to develop several future lines oriented specially to the improvement of the detection of analytes, but also to open novel ways for sensing applications. The main work lines that could be developed in the future might be:

- To use PS sensors, in particular PSRRs, for the detection of extremely low concentrations of BSA in order to compare the results with those presented in chapter 2 for the SOI RR. In particular, by studying the BSA sensitivity enhancement, the effective amount of field exploited in the sensing event can be determined and also the pore percentage covered by the bioreceptors.

- To increase the pore diameter of the PS sensors by using KOH. This advancement paves the way to biofunctionalize PS structures with more complex sensing techniques, 
including those based on the covalent binding, which require more steps and, so, the creation of more biolayers.

- To develop an interrogation platform able to perform a simultaneous measurement in different points of the 1D PS structure in order to perform a multiplexed detection. This line can be developed by fabricating a new fluidic cell where more optical fibers can be implemented in order to illuminate the PS sample and, thus, to realize a multiple detection at the same time.

- To develop a PS sensing probe suitable for bulk and biochemical sensing. To this aim, a PS membrane can be produced and placed on the optical fiber tip. In this way, we can have a porous face of the probe exposed to the target substance, which will be able to penetrate the PS structure in order to analyse i 


\section{Author publications}

\section{Journal Publications}

[1] Caroselli, R.; Sanchez, D. M.; Alcantara, S. P.; Quilez, F. P.; Moran, L. T.; GarciaRuperez, J. Experimental study of a real-time and highly sensitive fiber-optic porous silicon temperature sensing probe. IEEE Sens. J. 2018, 18(13), 5361-5367.

[2] Caroselli, R.; Castelló, J. G.; Escorihuela, J.; Bañuls, M. J.; Maquieira, Á.; GarcíaRupérez, J. Experimental study of the oriented immobilization of antibodies on photonic sensing structures by using protein A as an intermediate layer. Sensors 2018, 18(4), 1012.

[3] Caroselli, R.; Ponce-Alcántara, S.; Quilez, F. P.; Sánchez, D. M.; Morán, L. T.; Barres, A. G.; Bellieres, L.; Bandarenka, H.; Girel, K.; Bondarenko, V.; García-Rupérez, J. Experimental study of the sensitivity of a porous silicon ring resonator sensor using continuous in-flow measurements. Opt. Express 2017, 25(25), 31651-31659.

[4] Caroselli, R.; Martín Sánchez, D.; Ponce Alcántara, S.; Prats Quilez, F.; Torrijos Morán, L.; García-Rupérez, J. Real-Time and In-Flow Sensing Using a High Sensitivity Porous Silicon Microcavity-Based Sensor. Sensors 2017, 17(12), 2813. 


\section{Conference Publications}

[1] Caroselli, R.; Ponce-Alcántara, S.; Prats-Quílez, F.; Martín-Sánchez, D.; TorrijosMorán, L.; Griol Barres, A.; Bellieres , L,; Bandarenka, H.; Girel, K.; Bondarenko, V., García-Rupérez, J. Development and experimental study of a highly sensitive porous silicon ring resonator. European Confenrence on Integrated Optics - ECIO 2018, May 2018, Valencia, Spain.

[2] Caroselli, R.; Ponce-Alcántara, S.; Prats-Quílez, F.; Martín-Sánchez, D.; TorrijosMorán, L.; Griol Barres, A.; Bellieres , L,; Bandarenka, H.; Girel, K.; Bondarenko, V., García-Rupérez, J. In-flow and in-continuum refractive index sensing using a highly sensitive porous silicon ring resonator. ImagineNano 2018, March 2018, Bilbao, Spain.

[3] Caroselli, R.; Ponce-Alcántara, S.; Martín-Sánchez, D.; Torrijos-Morán, L.; GarcíaRupérez, J. Development and experimental study of a porous silicon fiber-optic temperature sensing probe. ImagineNano 2018, March 2018, Bilbao, Spain.

[4] García-Rupérez, J.; Ponce-Alcántara, S.; Ortiz de Zárate-Díaz, D.; Caroselli, R.; PratsQuílez, F.; Ruiz-Tórtola, Á.; Martín-Sánchez, D.; Martinez-Perez, P.; Nanophotonic Sensing Technology for High Sensitivity Analysis of Water Resources. International Symposium and Exhibition on Hydro-Environment Sensors and Software HydroSenSoft 2017, March 2017, Madrid, Spain.

[5] Prats, F.; Caroselli, R.; Ruiz-Tórtola, A.; García-Rupérez, J. High throughput interrogation platform for real time and highly multiplexed photonic detection using photonic bandgap structures. Proc. IEEE Sensors 2017, 5-7. 
[6] Caroselli, R.; Prats-Quílez, F.; Zurita, D.; Ruiz-Tórtola, Á.; García-Rupérez, J. Seminario Anual de Automática, Electrónica Industrial e Instrumentación - SAAEI16. July 2016, Elche, Spain.

[7] Caroselli, R.; Ponce-Alcántara, S.; Prats-Quílez, F.; García-Rupérez, J. Biosensors based on nanoporous silicon multilayer structures. V Conferencia Española de Nanofotónica - CEN 2016, June 2016, Valencia, Spain.

[8] Prats-Quílez, F.; Caroselli, R.; Zurita, D.; Ruiz-Tórtola, Á.; García-Rupérez, J. UltraFast Spectral Optical Analysis of Nanophotonic Structures. V Conferencia Española de Nanofotónica - CEN 2016, June 2016, Valencia, Spain.

[9] Bañuls, M. J.; García-Rupérez, J.; Caroselli, R.; Griol Barres, A; Puchades, R.; Maquieira, Á. PHOCNOSIS: a European project for early detection of cardiovascular diseases (CVD) based on label-free nanophotonic biosensing. Biosensors 2016, 2016, Gothenburg, Sweden

[10] García-Rupérez, J., Prats-Quílez, F.; Ruiz-Tórtola, Á.; Caroselli, R.; Griol Barres, A.; Hurtado Montañés, J. Plataforma fotónica de análisis para detección precoz de cáncer. Jornada Encuentro de Investigadores en Cáncer: 'dando la cara por la sociedad', March, 2016, Alcoi, Spain.

[11] Pla, L.; Caroselli, R.; Sancenón F., Martínez-Mañez R. Cocaine detection using gated mesoporous silicon films. 2nd International Symposium on Nanoparticles/Nanomaterials and Applications -2nd ISN2A 2016, January 2016, Caparica, Portugal.

[12] Caroselli, R.; Ponce-Alcántara, S.; Prats-Quílez, F.; Griol Barres, A.; Bellieres, L,; García-Rupérez, J. Nanoporous silicon photonic structures for the development of ultrasensitive biosensors. XVI Trends in Nanotechnology International Conference TNT2015, September 2015, Toulouse, France.

[13] Pla, L.; Caroselli, R.; Sancenón F.; Martínez-Mañez, R. Cocaine detection using gated mesoporous silicon films. IX International Workshop on Sensors and Molecular Recognition, July 2015, Valencia, Spain.

[14] Pizzoni, D.; Compagnone, D.; Griol Barres, A.; Acosta Romero, C.; Caroselli, R.; Barat Baviera, J. M. Deposition of peptide ligands on a gold nano-disks array and a silicon ring resonator. XVIII AISEM Annual Conference - AISEM 2015, February 2015, Trento, Italy. 
[15] Bañuls, M. J.; Gonzalez, D.; García-Rupérez, J.; Caroselli, R.; Griol Barres, A; Maquieira, Á.; SAPHELY: Proyecto europeo para el diagnóstico temprano de cancer basado en biosensado nanofotónico sin marcaje. IX International Workshop on Sensors and Molecular Recognition, July 2015, Valencia, Spain.

[16] Caroselli, R.; García-Rupérez, J. Development of an integrated photonic biosensor for specific biodetection. I Encuentro de Estudiantes de Doctorado de la Universitat Politècnica de València, June 2014, Valencia, Spain.

[17] Caroselli, R.; Castelló, J. G.; Escorihuela, J.; Bañuls, M. J.; Maquieira, Á.; GarcíaRupérez, J. Improvement of antigen detection using protein A for the oriented biofunctionalization of integrated photonic biosensor. IV Conferencia Española de Nanofotónica - CEN2014, May 2014, Santander, Spain.

[18] Mas Gomez, S. M.; Palací López, J.; Caroselli, R.; Zurita, D.; García-Rupérez, J. Monzón-Hernández, D.; Martí, J.; Refractive index sensor based on a curved biconical tapered fiber. IV Conferencia Española de Nanofotónica - CEN 2014, May 2014, Santander, Spain.

[19] Caroselli, R.; Castelló, J. G.; Escorihuela, J.; Bañuls, M. J.; Maquieira, Á.; GarcíaRupérez, J. Functionalization of integrated photonic biosensors with oriented antibodies using a protein A layer. XII European Conference on Optical Chemical Sensors and Biosensors Europtrode XII, April 2014. 


\section{References}

[1] García-Rupérez, J.; Toccafondo, V.; Bañuls, M.J.; Castelló, J.G.; Griol, A.; PeransiLlopis, S.; Maquieira, Á. Label-free antibody detection using band edge fringes in SOI planar photonic crystal waveguides in the slow-light regime. Opt. Express 2010, 18, 24276-24286.

[2] Toccafondo, V.; García-Rupérez, J.; Bañuls, M.J.; Griol, A.; Castelló, J.G.; PeransiLlopis, S.; Maquieira, A. Single-strand DNA detection using a planar photonic-crystalwaveguide-based sensor. Opt. Lett. 2010, 35, 3673-3675.

[3] Passaro, V.M.N.; Troia, B.; Notte, M. La; Leonardis, F. De Photonic resonant microcavities for chemical and biochemical sensing. RSC Adv. 2013, 3, 25-44.

[4] Scullion, M.G.; Krauss, T.F.; Di Falco, A. Slotted photonic crystal sensors. Sensors 2013, 13, 3675-3710.

[5] Lee, M.R.; Fauchet, P.M. Two-dimensional silicon photonic crystal based biosensing platform for protein detection. Opt. Express 2007, 15, 4530.

[6] Buswell, S.C.; Wright, V.A.; Buriak, J.M.; Van, V.; Evoy, S. Specific detection of proteins using photonic crystal waveguides. Opt. Express 2008, 16, 15949-15957.

[7] Castello, J.G.; Toccafondo, V.; Perez-Millan, P.; Losilla, N.S.; Cruz, J.L.; Andres, M. V; Garcia-Ruperez, J. Real-time and low-cost sensing technique based on photonic bandgap structures. Opt. Lett. 2011, 36, 2707-2709.

[8] Patko, D.; Cottier, K.; Hamori, A.; Horvath, R. Single beam grating coupled interferometry: High resolution miniaturized label-free sensor for plate based parallel screening. Opt. Express 2012, 20, 23162-23173.

[9] Huertas, C.S.; Fariña, D.; Lechuga, L.M. Direct and Label-Free Quantification of Micro-RNA-181a at Attomolar Level in Complex Media Using a Nanophotonic Biosensor. ACS Sens. 2016, 1, 748-756. 
[10] Estevez, M.C.; Alvarez, M.; Lechuga, L.M. Integrated optical devices for lab-on-a-chip biosensing applications. Laser Photonics Rev. 2012, 6, 463-487.

[11] Luchansky, M.S.; Bailey, R.C. High-Q optical sensors for chemical and biological analysis. Anal. Chem. 2012, 84, 793-821.

[12] Qavi, A.J.; Kindt, J.T.; Gleeson, M.A.; Bailey, R.C. Anti-DNA: RNA antibodies and silicon photonic microring resonators: Increased sensitivity for multiplexed microRNA detection. Anal. Chem. 2011, 83, 5949-5956.

[13] Š́́pová, H.; Zhang, S.; Dudley, A.M.; Galas, D.; Wang, K.; Homola, J. Surface plasmon resonance biosensor for rapid label-free detection of microribonucleic acid at subfemtomole level. Anal. Chem. 2010, 82, 10110-10115.

[14] Fan, X.; White, I.M.; Shopova, S.I.; Zhu, H.; Suter, J.D.; Sun, Y. Sensitive optical biosensors for unlabeled targets: A review. Anal. Chim. Acta 2008, 620, 8-26.

[15] Zinoviev, K.; Carrascosa, L. G.; Del Río, J. S.; Sepúlveda, B.; Domínguez, C.; Lechuga, L. M. Silicon photonic biosensors for lab-on-a-chip applications. Adv. Opt. Technol. 2008, 2008.

[16] Zanchetta, G.; Lanfranco, R.; Giavazzi, F.; Bellini, T.; Buscaglia, M. Emerging applications of label-free optical biosensors. Nanophotonics 2017, 6, 627-645.

[17] Gavela, A.F.; García, D.G.; Ramirez, J.C.; Lechuga, L.M. Last advances in siliconbased optical biosensors. Sensors 2016, 16, 285.

[18] Hunt, H.K.; Armani, A.M. Label-free biological and chemical sensors. Nanoscale 2010, 2,1544 .

[19] Vollmer, F.; Yang, L.; Fainman, S. Label-free detection with high-Q microcavities: A review of biosensing mechanisms for integrated devices. Nanophotonics 2012, 1, 267291.

[20] Peter, B.; Farkas, E.; Forgacs, E.; Saftics, A.; Kovacs, B.; Kurunczi, S.; Szekacs, I.; Csampai, A.; Bosze, S.; Horvath, R. Green tea polyphenol tailors cell adhesivity of RGD displaying surfaces: Multicomponent models monitored optically. Sci. Rep. 2017, $7,1-16$.

[21] Ciminelli, C.; Campanella, C. M.; Dell’Olio, F.; Campanella, C. E.; Armenise, M. N. Label-free optical resonant sensors for biochemical applications. Prog. Quantum Electron. 2013, 37, 51-107. 
[22] Escorihuela, J.; Bañuls, M.J.; Castelló, J.G.; Toccafondo, V.; García-Rupérez, J.; Puchades, R.; Maquieira, Á. Chemical silicon surface modification and bioreceptor attachment to develop competitive integrated photonic biosensors. Anal. Bioanal. Chem. 2012, 404, 2831-2840.

[23] Rusmini, F.; Zhong, Z.; Feijen, J. Protein immobilization strategies for protein biochips. Biomacromolecules 2007, 8, 1775-1789.

[24] Bañuls, M.J.; Puchades, R.; Maquieira, Á. Chemical surface modifications for the development of silicon-based label-free integrated optical (IO) biosensors: A review. Anal. Chim. Acta 2013, 777, 1-16.

[25] Ciminelli, C.; Dell'Olio, F.; Conteduca, D.; Campanella, C. M.; Armenise, M. N. High performance SOI microring resonator for biochemical sensing. Opt. Laser Technol. 2014, 59, 60-67.

[26] Pal, S.; Fauchet, P. M.; Miller, B. L. 1-D and 2-D photonic crystals as optical methods for amplifying biomolecular recognition. Anal. Chem. 2012, 84, 8900-8908.

[27] Bruck, R.; Melnik, E.; Muellner, P.; Hainberger, R.; Lämmerhofer, M. Integrated polymer-based Mach-Zehnder interferometer label-free streptavidin biosensor compatible with injection molding. Biosens. Bioelectron. 2011, 26, 3832-3837.

[28] Uhlir, A. Electrolytic Shaping of Germanium and Silicon. Bell Syst. Tech. J. 1956, 35, 333-347.

[29] Canham, L. T. Silicon quantum wire array fabrication by electrochemical and chemical dissolution of wafers. Appl. Phys. Lett. 1990, 57, 1046-1048.

[30] Jane, A.; Dronov, R.; Hodges, A.; Voelcker, N.H. Porous silicon biosensors on the advance. Trends Biotechnol. 2009, 27, 230-239.

[31] Bisi, O.; Ossicini, S.; Pavesi, L. Porous silicon: A quantum sponge structure for silicon based optoelectronics. Surf. Sci. Rep. 2000, 38, 1-126.

[32] Dhanekar, S.; Jain, S. Porous silicon biosensor: Current status. Biosens. Bioelectron. 2013, 41, 54-64.

[33] Mora, M. D. La; Ocampo, M.; Doti, R.; Lugo, J.; Faubert, J. Porous Silicon Biosensors. State Art Biosens. - Gen. Asp. 2013, 141-161. 
[34] Mariani, S.; Pino, L.; Strambini, L.M.; Tedeschi, L.; Barillaro, G. 10,000-fold improvement in protein detection using nanostructured porous silicon interferometric aptasensors. ACS Sens. 2016, 1, 1471-1479.

[35] Kim, H. J.; Kim, Y. Y.; Lee, K. W.; Park, S. H. A distributed Bragg reflector porous silicon layer for optical interferometric sensing of organic vapor. Sensors Actuators, $B$ Chem. 2011, 155, 673-678.

[36] Li, P.; Jia, Z.; Lü, X.; Liu, Y.; Ning, X.; Mo, J.; Wang, J. Spectrometer-free biological detection method using porous silicon microcavity devices. Opt. Express 2015, 23, 24626.

[37] Álvarez, J.; Bettotti, P.; Suárez, I.; Kumar, N.; Hill, D.; Chirvony, V.; Pavesi, L.; Martínez-Pastor, J. Birefringent porous silicon membranes for optical sensing. Opt. Express 2011, 19, 26106.

[38] Alvarez, J.; Bettotti, P.; Kumar, N; Suarez, I.; Hill, D.; Martinez-Pastor, J. Highlysensitive anisotropic porous silicon based optical sensors. Proc. SPIE 2012, 8212, 821209-1.

[39] Baratto, C.; Faglia, G.; Comini, E.; Sberveglieri, G.; Taroni, A.; La Ferrara, V.; Quercia, L.; Di Francia, G. A novel porous silicon sensor for detection of sub-ppm $\mathrm{NO}_{2}$ concentrations. Sens. Actuators B Chem. 2001, 77, 62-66.

[40] Anderson, M. A.; Tinsley-Bown, A.; Allcock, P.; Perkins, E. A.; Snow, P.; Hollings, M.; Smith, R. G.; Reeves, C.; Squirrell, D. J.; Nicklin, S.; Cox, T. I. Sensitivity of the optical properties of porous silicon layers to the refractive index of liquid in the pores. Phys. status solidi 2003, 197, 528-533, doi:10.1002/pssa.200306558.

[41] De Vos, K.; Bartolozzi, I.; Schacht, E.; Bienstman, P.; Baets, R. Silicon-on-Insulator microring resonator for sensitive and label-free biosensing. Opt. Express 2007, 15, 7610.

[42] Iqbal, M.; Gleeson, M. a; Spaugh, B.; Tybor, F.; Gunn, W. G.; Hochberg, M.; Baehrjones, T.; Bailey, R. C.; Gunn, L. C. Label-Free Biosensor Arrays Based on Silicon Scanning Instrumentation. IEEE J. Sel. Top. Quantum Electron. 2010, doi:10.1109/JSTQE.2009.2032510.

[43] Passaro, V. M. N.; Troia, B.; Notte, M. La; Leonardis, F. De Photonic resonant microcavities for chemical and biochemical sensing. RSC Adv. 2013, 3, 25-44. 
[44] Claes, T.; Molera, J. G.; De Vos, K.; Schacht, E.; Baets, R.; Bienstman, P. Label-free biosensing with a slot-waveguide-based ring resonator in silicon on insulator. IEEE Photonics J. 2009, 1, 197-204.

[45] Fard, S. T.; Donzella, V.; Schmidt, S. A.; Flueckiger, J.; Grist, S. M.; Talebi Fard, P.; Wu, Y.; Bojko, R. J.; Kwok, E.; Jaeger, N. A. F.; Ratner, D. M.; Chrostowski, L. Performance of ultra-thin SOI-based resonators for sensing applications. Opt. Express 2014, 22, 14166.

[46] Flueckiger, J.; Schmidt, S.; Donzella, V.; Sherwali, A.; Ratner, D. M.; Chrostowski, L.; Cheung, K. C. Sub-wavelength grating for enhanced ring resonator biosensor. Opt. Express 2016, 24, 15672.

[47] Hunt, H.K.; Armani, A.M. Label-free biological and chemical sensors. Nanoscale 2010, 2,1544 .

[48] Zourob, M.; Lakhtakia, A. Optical Guided-Wave Chemical and Biosensors II; Springer: Berlin, Germany, 2010; p. 270.

[49] Xu, D.-X.; Vachon, M.; Densmore, A.; Ma, R.; Delâge, A.; Janz, S.; Lapointe, J.; Li, Y.; Lopinski, G.; Zhang, D.; Liu, Q.Y.; Cheben, P.; Schmid, J.H. Label-free biosensor array based on silicon-on-insulator ring resonators addressed using a WDM approach. Opt. Lett. 2010, 35, 2771-3.

[50] De Vos, K.; Girones, J.; Claes, T.; De Koninck, Y.; Popelka, S.; Schacht, E.; Baets, R.; Bienstman, P. Multiplexed antibody detection with an array of silicon-on-insulator microring resonators. IEEE Photonics J. 2009, 1, 225-235.

[51] Luchansky, M.S.; Washburn, A.L.; Martin, T.A.; Iqbal, M.; Gunn, L.C.; Bailey, R.C. Characterization of the evanescent field profile and bound mass sensitivity of a labelfree silicon photonic microring resonator biosensing platform. Biosens. Bioelectron. 2010, 26, 1283-1291.

[52] Taniguchi, T.; Hirowatari, A.; Ikeda, T.; Fukuyama, M.; Amemiya, Y.; Kuroda, A.; Yokoyama, S. Detection of antibody-antigen reaction by silicon nitride slot-ring biosensors using protein G. Opt. Commun. 2016, 365, 16-23.

[53] Shang, J.; Cheng, F.; Dubey, M.; Kaplan, J.M.; Rawal, M.; Jiang, X.; Newburg, D.S.; Sullivan, P.A.; Andrade, R.B.; Ratner, D.M. An organophosphonate strategy for functionalizing silicon photonic biosensors. Langmuir 2012, 28, 3338-3344. 
[54] Van Laere, F.; Claes, T.; Schrauwen, J.; Scheerlinck, S.; Bogaerts, W.; Taillaert, D.; O'Faolain, L.; Van Thourhout, D.; Baets, R. Compact focusing grating couplers for silicon-on-insulator integrated circuits. IEEE Photonics Technol. Lett. 2007, 19, 19191921.

[55] Anderson, G.P.; Jacoby, M.A.; Ligler, F.S.; King, K.D. Effectiveness of protein A for antibody immobilization for a fiber optic biosensor. Biosens. Bioelectron. 1997, 12, 329-336.

[56] Wang, Z.; Jin, G. Feasibility of protein A for the oriented immobilization of immunoglobulin on silicon surface for a biosensor with imaging ellipsometry. $J$. Biochem. Biophys. Methods 2003, 57, 203-211.

[57] Meyer, V.K.; Kober, C.; Niessner, R.; Seidel, M. Regeneration of recombinant antigen microarrays for the automated monitoring of antibodies against zoonotic pathogens in swine sera. Sensors 2015, 15, 2614-2628.

[58] Julián, E.; Cama, M.; Martínez, P.; Luquin, M. An ELISA for five glycolipids from the cell wall of Mycobacterium tuberculosis: Tween 20 interference in the assay. $J$. Immunol. Methods 2001, 251, 21-30.

[59] Lebogang, L.; Mattiasson, B.; Hedström, M. Capacitive sensing of microcystin variants of Microcystis aeruginosa using a gold immunoelectrode modified with antibodies, gold nanoparticles and polytyramine. Microchim. Acta 2014, 181, 1009-1017.

[60] Barrios, C. A.; Bañuls, M. J.; González-Pedro, V.; Gylfason, K. B.; Sánchez, B.; Griol, A.; Maquieira, A.; Sohlström, H.; Holgado, M.; Casquel, R. Label-free optical biosensing with slot-waveguides. Opt. Lett. 2008, 33, 708, doi:10.1364/OL.33.000708.

[61] Grimaldi, I. A.; Testa, G.; Bernini, R. Flow through ring resonator sensing platform. RSC Adv. 2015, doi:10.1039/c5ra15117a.

[62] Li, Z.; Liao, C.; Chen, D.; Song, J.; Jin, W.; Peng, G.-D.; Zhu, F.; Wang, Y.; He, J.; Wang, Y. Label-free detection of bovine serum albumin based on an in-fiber MachZehnder interferometric biosensor. Opt. Express 2017, doi:10.1364/OE.25.017105.

[63] Pacholski, C. Photonic crystal sensors based on porous silicon. Sensors (Basel). 2013, 13, 4694-713, doi:10.3390/s130404694.

[64] Dhanekar, S.; Jain, S. Porous silicon biosensor: Current status. Biosens. Bioelectron. 2013, 41, 54-64, doi:10.1016/j.bios.2012.09.045. 
[65] Harraz, F.A. Porous silicon chemical sensors and biosensors: A review. Sens. Actuators B Chem. 2014, 202, 897-912, doi:10.1016/j.snb.2014.06.048.

[66] Snow, P.A.; Squire, E.K.; Russell, P.S.J.; Canham, L.T. Vapor sensing using the optical properties of porous silicon Bragg mirrors. J. Appl. Phys. 1999, 86, 1781-1784.

[67] Mulloni, V.; Pavesi, L. Porous silicon microcavities as optical chemical sensors. Appl. Phys. Lett. 2000, 76, 2523-2525.

[68] Lin, V. S. Y.; Motesharei, K.; Dancil, K. P. S.; Sailor, M. J.; Ghadiri, M. R. A porous silicon-based optical interferometric biosensor. Science, 1997, 278, 840-843.

[69] DeLouise, L.A.; Kou, P.M.; Miller, B.L. Cross-correlation of optical microcavity biosensor response with immobilized enzyme activity. Insights into biosensor sensitivity. Anal. Chem. 2005, 77, 3222-3230.

[70] Rendina, I.; Rea, I.; Rotiroti, L.; De Stefano, L. Porous silicon-based optical biosensors and biochips. Phys. E Low-Dimens. Syst. Nanostruct. 2007, 38, 188-192.

[71] Huanca, D.R.; Ramirez-Fernandez, F.J.; Salcedo, W.J. Porous silicon optical cavity structure applied to high sensitivity organic solvent sensor. Microelectron. J. 2008, 39, 499-506.

[72] Lasave, L.C.; Urteaga, R.; Koropecki, R.R.; Gonzalez, V.D.; Arce, R.D. Real-time study of protein adsorption kinetics in porous silicon. Colloids Surf. B Biointerf. 2013, $111,354-359$.

[73] Zhang, H.; Jia, Z.; Lv, X.; Zhou, J.; Chen, L.; Liu, R.; Ma, J. Porous silicon optical microcavity biosensor on silicon-on-insulator wafer for sensitive DNA detection. Biosens. Bioelectron. 2013, 44, 89-94.

[74] Zhao, Y.; Gaur, G.; Retterer, S.T.; Laibinis, P.E.; Weiss, S.M. Flow-through porous silicon membranes for real-time label-free biosensing. Anal. Chem. 2016, 88, 1094010948.

[75] Balili, R.B. Transfer matrix method in nanophotonics. Int. J. Mod. Phys. Conf. Ser. 2012, 17, 159-168.

[76] Pavesi, L. Porous silicon dielectric multilayers and microcavities. Riv. del Nuovo Cim. 1997, 20, 1-76. 
[77] Sohn, H. Refractive index of porous silicon. In Handbook of Porous Silicon; 2014, 231243.

[78] Bruggeman, D.A.G. Dielectric constant and conductivity of mixtures of isotropic materials. Ann. Phys. 1935, 24, 636-664.

[79] Korotcenkov, G. Porous Silicon: From Formation to Application, Volume Two; 2016

[80] Rasband, W.S. ImageJ, U.S. Available online: https://imagej.nih.gov/ij/.

[81] García-Rupérez, J.; Toccafondo, V.; Bañuls, M.J.; Castelló, J.G.; Griol, A.; PeransiLlopis, S.; Maquieira, Á. Label-free antibody detection using band edge fringes in SOI planar photonic crystal waveguides in the slow-light regime. Opt. Express 2010, 18, 24276-24286.

[82] Lin, H.; Gao, T.; Fantini, J.; Sailor, M. J. A porous silicon-palladium composite film for optical interferometric sensing of hydrogen. Langmuir 2004, 20, 5104-5108, doi:10.1021/la049741u.

[83] Orosco, M. M.; Pacholski, C.; Sailor, M. J. Real-time monitoring of enzyme activity in a mesoporous silicon double layer. Nat. Nanotechnol. 2009, 4, 255-258, doi:10.1038/nnano.2009.11.

[84] Pham, V. H.; Nguyen, T. Van; Nguyen, T. A.; Pham, V. D.; Bui, H. Nano porous silicon microcavity sensor for determination organic solvents and pesticide in water. Adv. Nat. Sci. Nanosci. Nanotechnol. 2014, 5, doi:10.1088/2043-6262/5/4/045003.

[85] Merzbacher, C. I.; Kersey, A. D.; Friebele, E. J. Fiber optic sensors in concrete structures: A review. Smart Mater. Struct. 1996, 5, 196-208.

[86] Lu, P.; Men, L.; Sooley, K.; Chen, Q. Tapered fiber Mach-Zehnder interferometer for simultaneous measurement of refractive index and temperature. Appl. Phys. Lett. 2009, 94.

[87] Lou, J.; Wang, Y.; Tong, L. Microfiber optical sensors: A review. Sensors 2014, 14, $5823-5844$.

[88] Lee, B. Review of the present status of optical fiber sensors. Opt. Fiber Technol. 2003, 9, 57-79.

[89] Kersey, A. D. A review of recent developments in fiber optic sensor technology. Opt. Fiber Technol. 1996, 2, 291-317. 
[90] Grattan, K. T. V; Sun, T. Fiber optic sensor technology: An overview. Sensors Actuators, A Phys. 2000, 82, 40-61.

[91] Spillman, W. B. Optical Detectors. In Fiber Optic Sensors: An Introduction for Engineers and Scientists: Second Edition, 2011, 63-86.

[92] Paliwal, N., John, J. Fiber Optic Sensors. In Fiber Optic Sensors, 2017, 21, 31-50.

[93] Yasin, M.; Harun, S. W.; Arof, H.; Ahmad, H. Fiber Optic Temperature Sensors. In Optical Fiber Communications and Devices, 2012, 361-380.

[94] Chiavaioli, F.; Trono, C.; Giannetti, A.; Brenci, M.; Baldini, F. Characterisation of a label-free biosensor based on long period grating. J. Biophotonics 2014, 7, 312-322.

[95] Wei, T.; Han, Y.; Li, Y.; Tsai, H.-L.; Xiao, H. Temperature-insensitive miniaturized fiber inline Fabry-Perot interferometer for highly sensitive refractive index measurement. Opt. Express 2008, 16, 5764.

[96] Lee, B. H.; Kim, Y. H.; Park, K. S.; Eom, J. B.; Kim, M. J.; Rho, B. S.; Choi, H. Y. Interferometric fiber optic sensors. Sensors 2012, 12, 2467-2486.

[97] Ahmad, H.; Chong, W. Y.; Thambiratnam, K.; Zulklifi, M. Z.; Poopalan, P.; Thant, M. M. M.; Harun, S. W.; Muang, M. High Sensitivity Fiber Bragg Grating Pressure Sensor Using Thin Metal Diaphragm. JSEN_IEEE Sensors J. 2009, 9, 1654-1659

[98] Liu, G.; Han, M.; Hou, W. High-resolution and fast-response fiber-optic temperature sensor using silicon Fabry-Pérot cavity. Opt. Express 2015, 23, 7237.

[99] Golnabi, H.; Kavei, M.; Azizi, K. Potentials of Plastic Optical Fibers for Sensor Technology. Adv. Mater. Res. 2008, 47-50, 161-164.

[100] Zhang, D.; Wang, J.; Wang, Y.; Dai, X. A fast response temperature sensor based on fiber Bragg grating. Meas. Sci. Technol. 2014, 25, 1-4.

[101] Nguyen, L. V.; Hwang, D.; Moon, S.; Moon, D. S.; Chung, Y. High temperature fiber sensor with high sensitivity based on core diameter mismatch. Opt. Express 2008, 16, 11369.

[102] James, S. W.; Tatam, R. P. Optical fibre long-period grating sensors: Characteristics and application. Meas. Sci. Technol. 2003, 14. 